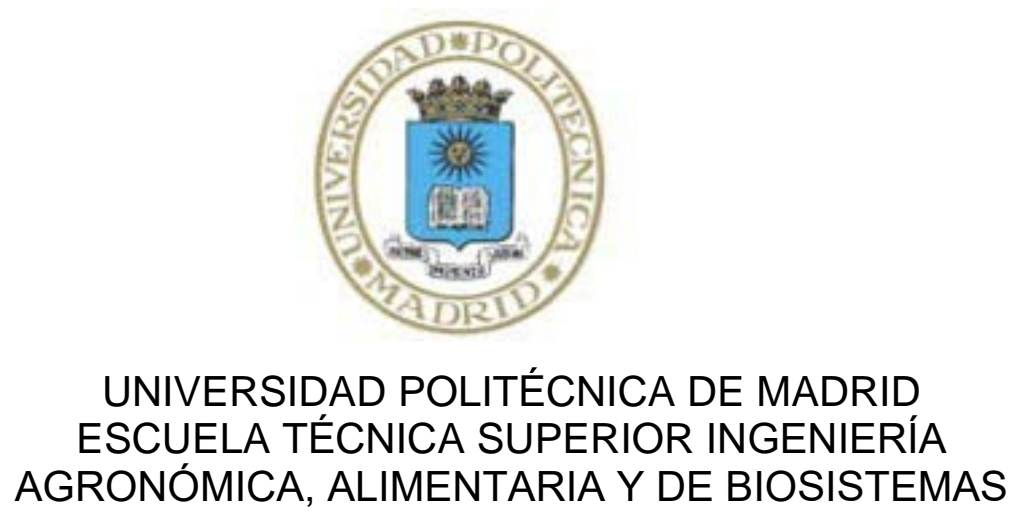

\title{
CARACTERIZACIÓN DE LA ESTRUCTURA DEL ESPACIO DE FASES DE SISTEMAS HAMILTONIANOS MEDIANTE INDICADORES DINÁMICOS
}

\author{
Pedro Benítez Gamero \\ Licenciado en CC Matemáticas
}

TESIS DOCTORAL

Julio de 2018 



\title{
CARACTERIZACIÓN DE LA ESTRUCTURA DEL ESPACIO DE FASES DE SISTEMAS HAMILTONIANOS MEDIANTE INDICADORES DINÁMICOS
}

\author{
Pedro Benítez Gamero
}

Licenciado en CC Matemáticas

Directores:

- Rosa Ma Benito Zafrilla Doctora en CC Químicas

- Juan Carlos Losada González Doctor en CC Físicas 



\section{Agradecimientos}

Mi primer agradecimiento debe ser para mis directores de tesis, Rosa M. Benito y Juan Carlos Losada. Es difícil ponderar lo que su apoyo, ayuda, comprensión y paciencia han supuesto para mí. Muchas gracias por haberos mostrado siempre tan dispuestos a ayudarme, a corregir los primeros borradores de la memoria, a la hora de hacer papeleo y por haberme animado los momentos difíciles. Que no han sido pocos

También quisiera expresar mi gratitud al resto de miembros del Grupo de Sistemas Complejos con los que he tenido el placer de compartir todos estos años: Fabio, Thiago, Antonio, Henar, Alfredo.

Y gracias también a Carlos que te atiende sin que les pidas nada y a Merce que bastante hace con poner orden entre tanto caótico desordenado.

Quisiera agradecer también a la Universidad Politécnica de Madrid, la posibilidad de haber elaborado esta tesis. En segundo lugar, quisiera dar las gracias al servicio de la biblioteca de la E.T.S.I.A.A.B y en particular a Teresa y a Ruth sin cuya ayuda esta tesis no habria visto la luz.

Y por supuesto, gracias a Carmen y a Pedro de cuya paciencia he abusado mucho más de lo que es normal sin que por ello perdieran la fe en el resultado final. 



\section{Resumen}

En el estudio de sistemas dinámicos que pueden ser descritos mediante una función hamiltoniana, los grados de libertad del sistema (también denominados dimensión del sistema) juegan un papel fundamental a la hora de enfocar su caracterización. Mientras que existen herramientas matemáticas bien definidas para visualizar y estudiar la estructura regular o caótica de sistemas de dos grados de libertad (2D), como por ejemplo la superficie de sección de Poincaré, en el caso de tres grados de libertad (3D), la situación es mucho más complicada y no está completamente resuelta. Esto es debido a que en este caso el espacio de fases tiene dimensión 6 o 5 si la energía total del sistema se conserva.

En esta tesis proponemos una metodología que permite caracterizar de forma completa el espacio de fases de sistemas hamiltonianos mediante indicadores dinámicos, que puede aplicarse independientemente de la dimensión del sistema.

Hemos usado como sistema hamiltoniano de estudio el sistema molecular LiNC/LiCN de dos y tres grados de libertad. En el modelo de dos grados de libertad se congela el movimiento $\mathrm{C}-\mathrm{N}$, mientras que en el modelo de tres grados de libertad se introduce un potencial Morse, que describe de manera realista la interacción entre los átomos de carbono y nitrógeno.

En el estudio del sistema 2D utilizamos el índice SALI (Small Alignment Index) para caracterizar la dinámica vibracional de nuestro sistema. Este índice se basa en analizar cómo se separan trayectorias cercanas mediante un algoritmo muy eficiente que es independiente de la dimensionalidad del sistema. Utilizando los valores numéricos mínimos de SALI en un periodo determinado de tiempo $\mathrm{T}$ (mSALIT), hemos podido realizar una completa caracterización de las diferentes dinámicas que aparecen en este sistema. En particular, hemos demostrado que SALI permite determinar la naturaleza regular o caótica de las trayectorias, así como diferenciar entre "caos fuerte" y "caos suave", distinguiendo el diferente comportamiento dinámico de trayectorias cercanas a órbitas periódicas y a cantoros. SALI resulta ser una poderosa herramienta en dinámica no lineal, que aporta información com- 
plementaria a las Superficies de Sección de Poincaré (SSP). Hemos definido diagramas y mapas, mediante diferentes aplicaciones que asignan un valor de SALI (en el intervalo $[0, \sqrt{2}]$ ) a cada condición inicial de un determinado subconjunto del espacio de fases. Para visualizar los distintos mapas hemos desarrollado visualizaciones en las que el valor de SALI se representa mediante un código de colores. Variando el subconjunto de condiciones iniciales sobre el espacio de fases, hemos construido el diagrama de bifurcación con SALI; las SSP con SALI (en los que se asigna el mismo valor de $m S A L I_{T}$ a los cortes correspondientes a la SSP de una misma trayectoria) y los mapas de SALI, donde las condiciones iniciales se encuentran sobre la SSP. Estos mapas nos han permitido obtener una visión global de la estructura del espacio de fases de nuestro sistema con dos grados de libertad.

En el estudio del sistema con tres grados de libertad se han generalizado los mapas de SALI utilizados previamente en el sistema 2D, incluyendo el tercer grado de libertad. Hemos desarrollado una metodología de visualizaciones $4 \mathrm{D}$, usando el color como una cuarta dimensión que representa el valor de $m S A L I_{T}$. Por un lado, partimos del plano correspondiente a la SSP del sistema de dos grados de libertad, añadimos una tercera dimensión correspondiente al nuevo grado de libertad y coloreamos cada punto en función de SALI. Mediante estas visualizaciones hemos podido caracterizar el espacio de fases del sistema con tres grados de libertad para diferentes valores de la energía vibracional total del sistema. Así mismo hemos demostrado que las zonas caóticas disminuyen cuando las condiciones iniciales implican mayor energía asociada al movimiento $\mathrm{C}-\mathrm{N}$, siendo una primera evidencia de que este movimiento está bastante desacoplado del resto. Hemos llevado a cabo un estudio de la proporción de zonas de caos y regularidad en función de la energía total del sistema tanto para el caso 2D como para el 3D. Hemos evidenciado que existe un rango de energías intermedias donde existen zonas de caos suave relacionadas con la existencia de estructuras fractales (cantoros) en el espacio de fases y que a altas energías siempre queda alrededor de un $6 \%$ de zona regular en el espacio de fases. Este valor se alcanza a energías más altas en el caso del sistema 3D. Además, en el caso de 3D el valor asintótico de la proporción de zonas de caos y de regularidad depende del valor inicial de la energía en el tercer grado de libertad, de forma que cuanta más energía vibracional inicial tiene, la proporción de trayectorias regulares es mayor. Esto es una nueva evidencia de que el movimiento en la nueva coordenada está muy desacoplado del movimiento del resto del sistema.

Otro indicador dinámico que hemos utilizado en el estudio del sistema 3D es el análisis de frecuencias. Este análisis ha demostrado ser un excelente indicador de caos que permite evidenciar la compleja estructura del espacio de fases de sistemas hamiltonianos, y en particular de sistemas moleculares, 
independientemente de su dimensión. En esta tesis se presenta un análisis sistemático de la molécula $\mathrm{LiCN}$ con tres grados de libertad, complementado, por primera vez en la literatura científica, con el índice SALI mediante visualizaciones 4D. Para ello, partimos del plano de relaciones de frecuencias obtenidas para condiciones iniciales sobre la SSP del sistema 2D, añadimos una tercera dimensión correspondiente al nuevo grado de libertad y coloreamos cada punto del espacio en función de su valor de $m S A L I_{T}$. Esto nos ha permitido caracterizar las diferentes zonas del espacio de fases en función de las relaciones de frecuencias entre los tres modos de vibración. Así, por ejemplo, dentro de la zona regular hemos podido encontrar toros de baja dimensionalidad, donde la relación entre dos frecuencias es un número racional, pero la relación del tercera frecuencia con las otras dos es un número irracional.

También hemos definido un nuevo mapa de SALI y su correspondiente visualización $4 \mathrm{D}$, que se basa en el estudio de las energías cinéticas medias, que complementa la información aportada por el análisis de frecuencias. De esta forma podemos mapear, usando visualizaciones $4 \mathrm{D}$, el espacio de fases en el espacio de frecuencias y en el espacio de energías, lo que nos permite obtener una visión completa de la estructura dinámica del sistema y realizar una correcta interpretación.

Hemos comprobado como para el sistema LiNC/LiCN la aproximación de dos grados de libertad es adecuada y hemos mostrado que la metodología desarrollada permite evidenciar, incluso en este caso, las nuevas estructuras que aparecen en los sistemas de tres grados de libertad, como por ejemplo los toros de baja dimensión.

Por último, hemos realizado un estudio exhaustivo del sistema de tres grados de libertad a una energía baja, para poder analizar en ausencia de movimiento caótico cómo influye el nuevo grado de libertad en la dinámica global del sistema. 



\section{Abstract}

In the study of dynamical systems that can be described by a Hamiltonian function, the degrees of freedom of the system (also called system dimension) play a fundamental role when it comes to characterize its dynamical behavior. While there are well-defined mathematical tools to visualize and study the regular or chaotic structure of systems of two degrees of freedom (2D), such as the Poincaré surface of section, in the case of three degrees of freedom (3D), the situation is much more complicated and is not completely resolved. This is because in this case the phase space has dimension 6 or 5 if the total energy of the system is conserved.

In this thesis we propose a methodology that allows to completely characterize the phase space of Hamiltonian systems by means of dynamic indicators, which can be applied independently of the dimension of the system.

We have used the LiNC / LiCN molecular system of two and three degrees of freedom as a case study of Hamiltonian systems. In the two degrees of freedom model, the C-N movement is frozen, whereas in the three degrees of freedom model, a Morse potential is introduced, which realistically describes the interaction between the carbon and nitrogen atoms.

In the study of the 2D system we use SALI (Small Alignment Index) to characterize the vibrational dynamics of our system. This index is based on analyzing how nearby trajectories are separated by a very efficient algorithm that is independent of the system's dimensionality. Using the minimum numerical values of SALI in a given period of time $T\left(m S A L I_{T}\right)$, we have been able to carry out a complete characterization of the different dynamical behaviors that appear in this system. In particular, we have shown that SALI allows to determine the regular or chaotic nature of trajectories, as well as to differentiate between "strong chao" and "soft chaos", distinguishing the different dynamic behavior of trajectories near periodic orbits and cantori. SALI turns out to be a powerful tool in non-linear dynamics, which provides complementary information to the Poincaré Section Surfaces (SSP).

We have defined diagrams and maps, by means of different applications that assign a value of SALI (in the interval $[0, \sqrt{2}]$ ) to each initial condition 
of a certain subset of the phase space. To visualize the different maps we have developed visualizations in which the value of SALI is represented by a color code. By varying the subset of initial conditions on the phase space, we have constructed the bifurcations diagram with SALI; the SSP with SALI (in which the same value of $m S A L I_{T}$ is assigned to the cuts corresponding to the SSP of the same trajectory) and the SALI maps, where the initial conditions are on the SSP. These maps have allowed us to obtain a global view of the structure of the phase space of our system with two degrees of freedom. In the study of the system with three degrees of freedom, the SALI maps previously used in the $2 \mathrm{D}$ system have been generalized, including the third degree of freedom. We have developed a $4 \mathrm{D}$ visualization methodology, using color as a fourth dimension that represents the $m S A L I_{T}$ value. On the one hand, we start from the plane corresponding to the SSP of the system of two degrees of freedom, we add a third dimension corresponding to the new degree of freedom and we color each point depending of its mSALIT value. Through these visualizations we have been able to characterize the phase space of the system with three degrees of freedom for different values of the total vibrational energy of the system. We have also shown that the chaotic zones decrease when the initial conditions imply higher energy associated with the C-N movement, being a first evidence that this vibrational mode is quite decoupled from the rest. We have carried out a study of the proportion of zones of chaos and regularity based on the total energy of the system for both the 2D and 3D cases. We have shown that there is a range of intermediate energies where there are zones of mild chaos related to the existence of fractal (cantorus) structures in the phase space and that at high energies there is always around $6 \%$ of the regular area in the phase space. This value is reached at higher energies in the case of the $3 \mathrm{D}$ system. Furthermore, in the case of $3 \mathrm{D}$, the asymptotic value of the proportion of chaos and regularity zones depends on the initial value of the energy in the third degree of freedom, so that the more initial vibrational energy it has, the proportion of regular trajectories is higher. This is new evidence that the motion in the new coordinate is very decoupled from the movement of the rest of the vibrational modes of the system.

Another dynamical indicator that we have used in the study of the 3D system is the frequency analysis. This analysis has proved to be an excellent indicator of chaos that makes it possible to demonstrate the complex structure of the phase space of Hamiltonian systems, and in particular of molecular systems, independently of their dimension. This thesis presents a systematic frequency analysis of the $\mathrm{LiCN}$ molecule with three degrees of freedom, complemented, for the first time in the scientific literature, with the SALI index through $4 \mathrm{D}$ visualizations. In order to do it, we start from 
the plane of frequency ratios obtained for initial conditions on the SSP of the $2 \mathrm{D}$ system, then we add a third dimension corresponding to the new degree of freedom and we color each space point according to its $m S A L I_{T}$ value. This has allowed us to characterize the different zones of the phase space as a function of the frequency ratios between the three vibrational modes. Thus, for example, within the regular zone we have been able to find low dimensionality tori, in which two frequencies have a rational ratio, but the third one has an irrational frequency ratio with the other two.

We have also defined a new SALI map and its corresponding 4D visualization, which is based on the study of average kinetic energies, which complements the information provided by the frequency analysis. In this way, by using 4D visualizations, we can map the phase space in the space of frequencies and in the space of energies, which allows us to obtain a complete vision of the dynamic structure of the system and to perform a correct interpretation.

We have verified that for the $\mathrm{LiNC} / \mathrm{LiCN}$ system the approximation of two degrees of freedom is adequate and we have shown that the methodology developed allows to demonstrate, even in this case, the new structures that appear in the system of three degrees of freedom, such as the low dimension tori.

Finally, we have carried out an exhaustive study of the system of three degrees of freedom at a low energy level, in order to analyze, in the absence of chaotic movement, how the new degree of freedom influences the global dynamics of the system. 



\section{Índice general}

1. Introducción 1

I TEORÍA

2. Estudio de los sistemas Hamiltonianos $\quad 7$

2.1. Sistemas Hamiltonianos . . . . . . . . . . . . . 7

2.1.1. Formalismo de Lagrange . . . . . . . . . . . . . 7

2.1.2. Formalismo de Hamilton . . . . . . . . . . . . . . 9

2.1.3. Transformaciones Canónicas . . . . . . . . . . . . 11

2.1.4. Notación Simpléctica . . . . . . . . . . . . . . . . . 13

2.1.5. El Teorema de Liouville . . . . . . . . . . . . . . 14

2.1.6. Corchetes de Poisson . . . . . . . . . . . . . . . . 15

2.1.7. Sistemas Integrables . . . . . . . . . . . . 16

2.2. Sistemas de un grado de libertad . . . . . . . . . . . . . . 19

2.2.1. Péndulo simple . . . . . . . . . . . . . . . . . . . . . 19

2.2.2. Oscilador armónico . . . . . . . . . . . . . . . 20

2.3. Sistemas de dos o más grados de libertad . . . . . . . . . . . 21

2.3.1. Sistemas integrables de dos grados o más grados de libertad . . . . . . . . . . . . . 21

2.3.2. Sistemas no integrables. Caos . . . . . . . . . . 23

2.3.3. Teorema KAM . . . . . . . . . . . . . . 24

2.4. Dinámica linealizada . . . . . . . . . . . . . . . 26

2.5. Estabilidad de las órbitas periódicas . . . . . . . . . . . 27

3. Indicadores del caos $\quad 29$

3.1. Superficie de Sección de Poincaré . . . . . . . . . . . . . . 29

3.2. Exponentes de Lyapunov (LCE) . . . . . . . . . . . . . . . . . . 31

3.3. Análisis de Frecuencias . . . . . . . . . . . . . . . . . . . . . 32

3.4. Small Allignement Indicator (SALI) . . . . . . . . . . . . 36 
4. Sistema Molecular LiNC/LiCN 39

4.1. Descripción General . . . . . . . . . . . . . . . . . . . 39

4.2. Geometría del sistema y descripción de su movimiento . . . . 40

4.3. Descripción del Hamiltoniano . . . . . . . . . . . . . . . . 41

4.3.1. Potencial del Sistema 2D . . . . . . . . . . . 42

4.3.2. Potencial del sistema 3D . . . . . . . . . . . . . 45

4.4. Ecuaciones de Hamilton y Ecuaciones de Variación . . . . . . 47

4.4.1. Ecuaciones de Hamilton del LiNC/LiCN . . . . . . . . . . 47

4.4.2. Ecuaciones de Variación . . . . . . . . . . . . . . 47

II RESULTADOS EN 2D 49

5. Resultados del sistema de dos grados de libertad 51

5.1. Reducción a dos grados de libertad . . . . . . . . . . . . . 51

5.2. Superficies de sección de Poincaré y Mapas de SALIs . . . . . 53

5.2.1. Superficie de sección de Poincaré . . . . . . . . . . . 53

5.2.2. Mapas de SALI . . . . . . . . . . . . . . . . 54

5.3. Resultados . . . . . . . . . . . . . . . 56

5.3.1. Superficies de sección de Poincaré y SALI . . . . . . 56

5.3.2. Evolución temporal del indicador SALI . . . . . . . . 58

5.3.3. Zonas de regularidad y caos en función de la energía . . 66

III RESULTADOS EN 3D 73

6. Sistema con tres grados de libertad $\quad 75$

6.1. Mapas del sistema en 3D . . . . . . . . . . . . . . 76

6.1.1. Mapa SM en 3D $\left(S M_{3 D}\right) \ldots \ldots \ldots$. . . . . . 76

6.1.2. Mapa DBS en 3D $\left(D B S_{3 D}\right) \ldots \ldots \ldots . \ldots 76$

6.1.3. Mapa de Frecuencias (MF) . . . . . . . . . . . 77

6.1.4. Mapa de Frecuencias-SALI (MFS) . . . . . . . . . . . 78

6.1.5. Mapa de Energía Media $\left(M E M_{3 D}\right) \ldots \ldots . . . . .78$

6.1.6. Mapa de Frecuencia Energía $(M F E)$. . . . . . . . . . 79

6.2. Evolución de la regularidad y el caos con la Energía . . . . . . 80

6.2.1. Mapa DBS-SALI . . . . . . . . . . . . . 80

6.2.2. Mapa SALI-SM $S M_{3 D} \ldots \ldots \ldots$. . . . . . . . . 82

6.2.3. Mapas de Frecuencias con SALI $\left(M F S_{3 D}, M F S_{3 D}\right)$. . 85

6.2.4. Mapa de Energía Media $\left(M E M_{3 D}\right) \ldots . . . . . .87$

6.2.5. Mapa de Frecuencia Energía $(M F E)$. . . . . . . . . 90 
6.2.6. Distribución del porcentaje de caos en función de la energía ....................... 93

6.3. Comparación entre los modelos 2D y 3D . . . . . . . . . . 95

6.4. SUMARIO . . . . . . . . . . . . . . . . . . . . . . 98

7. Estudio detallado del $\operatorname{LiNC} / \operatorname{LiCN}$ a baja energía 99

7.1. Mapas 3D y Visualización 4D . . . . . . . . . . . . . . . . . 99

7.1.1. Mapa $S M_{3 D} \ldots \ldots \ldots$. . . . . . . . . . . . 100

7.1.2. Mapa $M F E_{3 D} \quad \ldots \ldots \ldots 102$

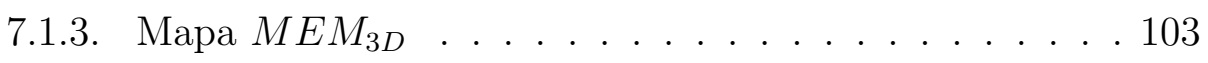

7.2. Clasificación de las órbitas estudiadas . . . . . . . . . . . . . . 104

7.2.1. Órbitas en la resonancia $(0,1,-2) \ldots \ldots$. . . . . . 108

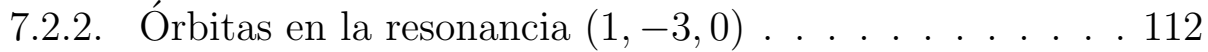

7.2.3. Órbitas en la resonancia $(0,1,-17) \ldots \ldots . . . . .116$

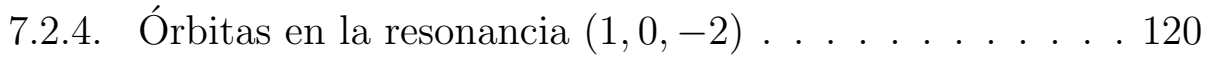

7.2.5. Órbitas en la resonancia $(1,0,-6) \ldots . . . . . . .124$

7.2.6. Órbitas en la resonancia $(0,1,-18) \ldots \ldots . .728$

7.3. SUMARIO. . . . . . . . . . . . . . . . . 138

\section{CONCLUSIONES $\quad 139$}

8. Conclusiones 141

A. Energía Media 151

A.1. Cálculo de la Energía Media . . . . . . . . . . . . . . . . 151

A.2. Relación entre la energía media y la acción . . . . . . . . . . . 152

A.3. Unicidad de la energía media de las trayectorias . . . . . . . 153

B. Comportamiento Asintótico de SALI 155

C. Detalle de implementación 163

C.1. Descripción del Sistema . . . . . . . . . . . . . . . 163

C.2. Integrador . . . . . . . . . . . . . . . . 163

C.3. Indicadores . . . . . . . . . . . . . . . . . . . . . 164

C.4. Aplicaciones . . . . . . . . . . . . . . . . . . . . 164

C.5. Graficos . . . . . . . . . . . . . . . 165 



\section{Índice de figuras}

2.1. Izquierda: Péndulo Simple. Derecha: Espacio de Fases correspondiente, en rojo los movimientos de libración y en verde los de rotación, la linea de trazos negra es la separatriz . . . . . . 20

2.2. Trayectorias dentro de un toro para una relación de frecuencias $\omega_{1} / \omega_{2}$ (a) racional y (b) irracional . . . . . . . . . . . 22

3.1. Trayectorias (izquierda) y sus SSP correspondientes (derecha). Las trayectorias son Periodicas (arriba), Cuasiperiodicas (medio) y Caóticas (abajo) . . . . . . . . . . . 30

3.2. Evolución en el espacio de fases de dos trayectorias cercanas. . 32

3.3. Mapa de frecuencias del $\mathrm{LiCN} / \mathrm{LiNC} 2 \mathrm{D}$ a la energía 2549.2 $\mathrm{cm}^{-1}$. Se representa la relación entre las frecuencias de $\mathrm{R}$ y de

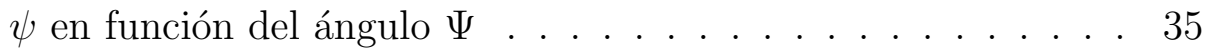

3.4. Ilustración grafica sobre la forma de la suma y la diferencia de dos vectores cercanos a la trayectoria de referencia, $\vec{z}(t)$. Aplicación al calculo de SALI . . . . . . . . . . . 36

3.5. Test de la validez de la aproximación $\operatorname{sali}(t)=e^{-\lambda t}$ En la izquierda primeros coeficientes de Lyapunov $\sigma_{1}, \sigma_{2}$ de una órbita asociada al Hamiltoniano $\left.H=\sum_{i=1}^{3} \frac{1}{2}\left(q_{i}^{2}+p_{i}^{2}\right)+q_{1}^{2} q_{2}+q^{2} q_{3}\right)$. A la derecha representación de $-\log (\operatorname{sali}(t)$ y de la recta $s=$ $-\frac{\left(\sigma_{1}-\sigma_{2}\right) t}{\ln ()}+C$ en función del tiempo . . . . . . . . . 38

4.1. Coordenadas de Jacobi para el sistema molecular usadas para describir los movimientos relativos internucleares vibracionales, es decir no se tiene en cuenta el movimiento puramente translacional y se suponen estados no excitados rotacionalmente 42

4.2. Curvas de nivel del potencial $V(R, \theta)$ correspondiente al sistema $L i C N$ de dos grados de libertad. La linea de trazos representa el camino de mínima energía que conecta los dos pozos . 44

4.3. Perfil del camino de mínima energía correspondiente al sistema $L i N C / L i C N \ldots \ldots \ldots \ldots \ldots$. . . . . . . . . . . . . 45 
4.4. Representación del potencial de Morse entre los átomos $\mathrm{C}$ y $\mathrm{N}$ con los valores dados en la expresión 4.9 . . . . . . . . . 46

5.1. Superficies de Sección de Poincaré compuestas (SSP) (izquierda) y los correspondientes mapas SALI-SSP (derecha ) para diferentes valores de la Energía vibracional: $1510,5 \mathrm{~cm}^{-1}$ (arriba), $2549,2 \mathrm{~cm}^{-1}$ (en medio), y $3823,8 \mathrm{~cm}^{-1}$ (abajo). La escala logarítmica de colores, correspondientes a $m S A L I$, se exhibe también en la esquina inferior derecha la figura. . . . . . . . 56

5.2. Detalle del panel medio de la figura 5.1. Posiciones iniciales (A-D) de las cuatro órbitas discutidas en el texto (Véase la tabla 5.1$) \ldots \ldots \ldots \ldots \ldots \ldots$

5.3. Órbita en el espacio de configuración (Fila superior), superficie de sección de Poincaré (Segunda fila), evolución temporal de $S A L I$ (tercera fila) y su versión promediada, $\overline{S A L I}$, de las cuatro trayectorias A-D marcadas en la figura 5.2 (Véase también la tabla 5.1 y el texto para los detalles). . . . . . . . . 59

5.4. Evolución temporal de $S A L I$ de la trayectoria caótica C mostrada en la figura 5.2 y cuyas características se describen en la tabla 5.1 para tiempos largos: $T=1,3 \cdot 10^{6}$ ua. . . . . . . 61

5.5. Órbita en el espacio de configuración (Arriba), SSP (Fila central), y evolución temporal de $\overline{S A L I}_{T}$ (Abajo) para diferentes porciones de la trayectoria $\mathrm{C}$ exhibida en la figura 5.4. . . . .

5.6. Detalle de la superficie de sección de Poincaré compuesta del LiNC/LiCN a $E=2549,2 \mathrm{~cm}^{-1}$ presentado en la figura 5.2 cerca de la trayectoria B. . . . . . . . . . . . . . .

5.7. Evolución temporal de $\overline{S A L I}$ (arriba izquierda) para las órbitas: estable , $S$ (línea roja continua) y su entorno $S^{\prime}$ (línea verde discontinua) y $S^{\prime \prime}$ (línea azul punteada) marcadas en la figura 5.6. Véase el texto para los detalles. Las órbitas $S, S^{\prime}$ y $S^{\prime \prime}$ se han dibujado en el espacio de configuración. . . . . . . 64

5.8. Evolución temporal de $\overline{S A L I}$ para las órbitas: inestable $U$, (línea roja continua) y su entorno $U^{\prime}$, (línea verde discontinua) y $U^{\prime \prime}$, (línea azul punteada) marcadas en la figura 5.6. Véase el texto para los detalles. Las órbitas $U, U^{\prime}$ y $U^{\prime \prime}$ se han dibujado en el espacio de configuración. . . . . . . . . . . . . . 
5.9. Diagrama de Bifurcación-continuación coloreado con SALI para el LiNC (Mapa DBS-SALI) como hemos definido en la sección 5.2.2. Las condiciones iniciales $\Omega_{1}$ dadas en la ecuación (5.10) son $\Delta R=2,2 \cdot 10^{-3}$ u.a. y $\Delta E=7,0 \mathrm{~cm}^{-1}$; Las trayectorias han sido propagadas hasta $T=2,5 \cdot 10^{5}$ u.a. para obtener los valores de $m S A L I_{T}$ en el gráfico usando la escala de colores incluida en la figura. Se muestra también el diagrama usual bifurcación-continuación de órbitas periódicas superpuesto en negro. . . . . . . . . . . . . . . . .

5.10. Mapas SM (columna izquierda) del sistema $\operatorname{LiNC} / \operatorname{LiCN}$ en $3 \mathrm{D}$ a las energías de $E=1000 \mathrm{~cm}^{-1}, 2000 \mathrm{~cm}^{-1}, 3000 \mathrm{~cm}^{-1}$, y $4000 \mathrm{~cm}^{-1}$ mostrados en los paneles $A-B-C-D$ respectivamente. La malla de condiciones iniciales $\Omega_{2}((5.10))$ es $\Delta \psi_{0}=1,570 \cdot 10^{-2}$ rad y $\Delta P_{\psi}^{0}=0,4$ u.a. Las trayectorias han sido propagadas durante $T=2,5 \cdot 10^{5}$ u.a. para obtener los valores de $m S A L I_{T}$ en el gráfico usando la escala de colores que se incluye en la figura. Los histogramas correspondientes de $m S A L I_{T}$ están dibujados en la columna derecha. Téngase en cuenta que el último pico del panel D incluye todos los valores de SALI menores que $10^{-19}$, y no solo al intervalo $\left(10^{-19}-10^{-20}\right)$ debido a la precisión limitada de los cálculos. . 68

5.11. Fracción de las tres regiones del espacio de fase del $\mathrm{LiNC} / \mathrm{LiCN}$ correspondientes a: Movimiento Regular (triángulos naranja) $\left(m S A L I_{T}>10^{-6}\right)$, Caos Suave (cuadrados verdes) $\left(10^{-6}>\right.$ $\left.m S A L I_{T}>10^{-16}\right)$, y Caos Fuerte (círculos azules) $\left(m S A L I_{T}<\right.$ $\left.10^{-16}\right)$. La línea vertical (púrpura) en $2281 \mathrm{~cm}^{-1}$ indica la energía en la que la región del pozo del isómero Li-CN comienza a ser accesible y puede existir movimiento en dicha zona, la línea vertical (verde) en $2754 \mathrm{~cm}^{-1}$ corresponde la energía inicial a la cual hay una compensación entre la destrucción de toros regulares y la aparición de nuevas regiones de regularidad. La línea vertical (negra) en $3450 \mathrm{~cm}^{-1}$ corresponde a la energía de la barrera de energía potencial para la reacción de isomerización. 
6.1. Visualizaciones del mapa $D B S_{3 D}$ del $\operatorname{LiNC} / \operatorname{LiCN}$ en $3 \mathrm{D}$. (a) Mapa 3D; (b) corte con el plano $P_{r}=0$ (c), corte con el plano $R=4,4$ u.a.; (d) corte con el plano $\mathbf{E}=3500 \mathrm{~cm}^{-1}$. Las condiciones iniciales $\Omega_{3 D}$ de acuerdo con la ecuación (6.2) se han obtenido seleccionando de forma aleatoria valores para $R \in[3,5]$ u.a, $P_{r} \in[-40,40]$ u.a. y $E \in[500,3500] \mathrm{cm}^{-1}$. Las trayectorias han sido propagadas durante $T=7,5 \cdot 10^{5}$ u.a. para obtener los valores de $m S A L I_{T}$ que se representan en el gráfico usando la escala de colores incluida en la figura. . . . .

6.2. Mapas de SALI-SM (columna izquierda) y cortes con los planos $P_{\psi}=0$ (columna central) y $P_{r}=0$ (columna derecha) a las energías de $E=1000 \mathrm{~cm}^{-1}, 2000 \mathrm{~cm}^{-1}, 3000 \mathrm{~cm}^{-1}, \mathrm{y}$ $4000 \mathrm{~cm}^{-1}$. La condiciones iniciales $z_{0} \in \Omega_{3 D}$ se han obtenido según la definición 6.1.1. Las trayectorías han sido propagadas durante $T=7,5 \cdot 10^{5}$ u.a. para obtener los valores de $m S A L I_{T}$ en el gráfico usando la escala de colores que se incluye en la figura. . . . . . . . . . . . . . . . 8

6.3. Evolución con la energía del mapa $S M_{3 D}$ (izquierda) y del mapa de de frecuencias Mapa $M F S_{2 D}$ (derecha) para el $L i N C / L i C N$ 3D como hemos definido en la sección 6.1. La condiciones iniciales $z_{0} \in \Omega_{3 D}$ se han obtenido de acuerdo con 6.1.1. Las trayectorias han sido propagadas durante $T=7,5 \cdot 10^{5}$ u.a. para obtener los valores de $m S A L I_{T}$ en el gráfico usando la escala de colores que se incluye en la figura. . . . . . . . . . 86

6.4. Evolución con la energía de los diagramas corrrespondientes a los mapas $S M_{3 D}$ y de energía media, $M E M_{3 D}$, coloreados con SALI para el $L i N C / L i C N$ en 3D como hemos definido en la sección 6.1. La condiciones iniciales $z_{0} \in \Omega_{3 D}$ se han obtenido seleccionando de forma aletoria valores para $\psi, P_{\psi} \mathrm{y}$ $P_{r}$ obteniendose el resto de los valores de acuerdo con 6.1.1. Las trayectorías han sido propagadas durante $T=7,5 \cdot 10^{5}$ u.a. para obtener los valores de $m S A L I_{T}$ en el gráfico usando la escala de colores que se incluye en la figura. . . . . . . . . . 89 
6.5. Evolución con la energía de los diagramas corrrespondientes a los mapas $S M_{3 D}$ y de frecuencia energía, $M F E$, coloreados con SALI para el $\mathrm{LiNC} / \mathrm{LiCN}$ en 3D como hemos definido en la sección 6.1. La condiciones iniciales $z_{0} \in \Omega_{3 D}$ se han obtenido seleccionando de forma aletoria valores para $\psi, P_{\psi}$ y $P_{r}$ obteniendose el resto de los valores de acuerdo con 6.1.1. Las trayectorías han sido propagadas durante $T=7,5 \cdot 10^{5}$ u.a. para obtener los valores de $m S A L I_{T}$ en el gráfico usando la escala de colores que se incluye en la figura. . . . . . . . . . 92

6.6. Fracción de las tres regiones del espacio de fase del LiNC/LiCN correspondientes a: Movimiento Regular (triángulos rojas) $\left(m S A L I_{T}>\right.$ $\left.10^{-6}\right)$, Caos Suave (cuadrados verdes) $\left(10^{-6}>m S A L I_{T}>\right.$ $\left.10^{-16}\right)$, y Caos Fuerte (círculos azules) $\left(m S A L I_{T}<10^{-16}\right)$. La línea vertical (púrpura) en $2281 \mathrm{~cm}^{-1}$ indica la energía en la que la región del pozo del isómero Li-CN comienza a ser accesible y puede existir movimiento en dicha zona, la línea vertical (verde) en $2754 \mathrm{~cm}^{-1}$ corresponde la energía inicial a la cual hay una compensación entre la destrucción de toros regulares y la aparición de nuevas regiones de regularidad. La línea vertical (negra) en $3450 \mathrm{~cm}^{-1}$ corresponde a la energía de la barrera de energi'a potencial para la reacción de isomerización. 93

6.7. Comparación entre la distribución de orbitas caóticas en 2D (izquierda) y 3D (derecha) . . . . . . . . . . . 96

6.8. Porcentaje de órbitas caóticas en 3D en función de la energía y del valor de $P_{r} \ldots \ldots \ldots \ldots$. . . . . . . . . . . . . . 96

7.1. Visualizaciones $4 \mathrm{D}$ de los mapas $S M_{3 D}$ (izquierda) $M F E_{3 D}$ (centro) y $M E M_{3 D}$ (derecha) a la energía de $512 \mathrm{~cm}^{-1}$. El color indica el valor de $m S A L I_{T}$. . . . . . . . . . . . . 100

7.2. Visualización del mapa $S M_{3 D}$ en $3 \mathrm{D}$ a la energía de $512,0 \mathrm{~cm}^{-1}$ en 3D (izquierda) y cortes del mismo para distintos valores de $P_{r}$ (derecha). El valor de $-\log _{10}\left(m S A L I_{t}\right.$ se muestra con un codigo de colores incluido en la figura . . . . . . . . . . 101

7.3. Visualización $4 \mathrm{D}$ del diagrama correspondiente al mapa $M F E_{3 D}$ (izquierda) y cortes del mismo con planos con un valor de $E r$ definido (derecha). . . . . . . . . . . . . . . . 102

7.4. Mapa de energía cinética media de las órbitas estudiadas a 512 $\mathrm{cm}^{-1}$ (izquierda). Cortes del diagrama 3D del mapa $M E M_{3 D}$ con planos con un valor de $E r$ definido (derecha). . . . . . . . 103

7.5. Clasificación de las órbitas estudiadas en el mapa $M F E_{3 D}$ (izquierda) y en el mapa $M E M_{3 D}$ (derecha), véase la tabla 7.1. 104 
7.6. Grupo (a). Órbitas ligadas a la resonancia $(0,1,-2)$ entre $r$ y $\theta$. Se muestra la posición de las órbitas con puntos negros en los distintos mapas: $S M_{3 D}$ (izquierda), $M F E_{3 D}$ (centro) y $M E M_{3 D}$ (derecha). . . . . . . . . . . . . . . 108

7.7. Descripción detallada de la órbita a1. . . . . . . . . . . . . . . 109

7.8. Descripción de la órbita a2. . . . . . . . . . . . . . . . 110

7.9. Descripción de la órbita a3. . . . . . . . . . . . . . . . 111

7.10. Grupo (b). Órbitas ligadas a la resonancia $(1,-3,0)$ entre $R$ y $r$. Se muestra la posición de las órbitas con puntos negros en los distintos mapas: $S M_{3 D}$ (izquierda), $M F E_{3 D}$ (centro) y $M E M_{3 D}$ (derecha). . . . . . . . . . . . . . . 112

7.11. Descripción de la órbita b1. . . . . . . . . . . . . . . . . 113

7.12. Descripción de la órbita b2. . . . . . . . . . . . . . . . . 114

7.13. Descripsción de la órbita b3. . . . . . . . . . . . . . . . . 115

7.14. Grupo (c). Órbitas ligadas a la resonancia $(0,1,-17)$ entre $r$ y $\theta$.Se muestra la posición de las órbitas con puntos negros en los distintos mapas: $S M_{3 D}$ (izquierda), $M F E_{3 D}$ (centro) y $M E M_{3 D}$ (derecha) . . . . . . . . . . . . . 116

7.15. Descripción de la órbita c1. . . . . . . . . . . . . 117

7.16. Descripción de la órbita c2. . . . . . . . . . . . . . . . 118

7.17. Descripción de la órbita c3. . . . . . . . . . . . . . . . . . 119

7.18. Grupo (d). Órbitas ligadas a la resonancia $(1,0,-2)$ entre $R$ y $\theta$.Se muestra la posición de las órbitas con puntos negros en los distintos mapas: $S M_{3 D}$ (izquierda), $M F E_{3 D}$ (centro) y $M E M_{3 D}$ (derecha). . . . . . . . . . . . . 120

7.19. Descripción de la órbita d1. . . . . . . . . . . . . . . 121

7.20. Descripción de laórbita d2. . . . . . . . . . . . . . . . . 122

7.21. Descripción de la órbita d3. . . . . . . . . . . . . . . . 123

7.22. Grupo (e). Órbitas ligadas a la resonancia $(1,0,-6)$ entre $R$ y $\theta$.Se muestra la posición de las órbitas con puntos negros en los distintos mapas: $S M_{3 D}$ (izquierda), $M F E_{3 D}$ (centro) y $M E M_{3 D}$ (derecha). . . . . . . . . . . . . . 124

7.23. Descripción de la órbita e1. . . . . . . . . . . . . . . 125

7.24. Descripción de la órbita e2. . . . . . . . . . . . . . . 126

7.25. Descripción de la órbita e3. . . . . . . . . . . . . . . . . . 127

7.26. Grupo (f). Órbitas ligadas a la resonancia $(0,1,-18)$ entre $r$ y $\theta$.Se muestra la posición de las órbitas con puntos negros en los distintos mapas: $S M_{3 D}$ (izquierda), $M F E_{3 D}$ (centro) y $M E M_{3 D}$ (derecha). . . . . . . . . . . . . . . . . 128

7.27. Descripción de la órbita f1. . . . . . . . . . . . . . . . . . . . 129

7.28. Descripción de la órbita f2 . . . . . . . . . . . . . . . 130 
7.29. Descripción de la órbita f3. . . . . . . . . . . . . . 131

7.30. Grupo (g). Órbitas ligadas a la resonancia $(2,-6,1)$. . . . . 132

7.31. Descripción de la órbita g1. . . . . . . . . . . . 133

7.32. Descripción de la órbita g2. . . . . . . . . . . . . . . . 134

7.33. Descripción de la órbita g3. . . . . . . . . . . . . . 135

7.34. Grupo (h). Órbita en la resonancia $(1,1,-2)$. . . . . . . 136

7.35. Descripción de la órbita h1. . . . . . . . . . . . 137

B.1. Vectores de desviación. Detalle de la figura 3.4 . . . . . . . 155 



\section{Índice de cuadros}

4.1. Configuración y energías de los puntos relevantes de la superficie de energía potencial [31] usada en el modelo de dos grados de libertad del sistema molecular $\mathrm{LiNC} / \mathrm{LiCN}$. . . . . . . . 44

5.1. Condiciones iniciales y características dinámicas de las cuatro trayectorias marcadas $(\mathrm{A}-\mathrm{D})$ en la figura 5.2 y mostradas en la figura 5.3. . . . . . . . . . . . . . . . . . . . 59

7.1. Grupos de órbitas seleccionadas a $512,0 \mathrm{~cm}^{-1}$ en la figura 7.5106

C.1. Comparativa de Métodos de Integración . . . . . . . . . . . 164 



\section{Capítulo 1}

\section{Introducción}

Una gran mayoría de los problemas de interés en Física involucran ecuaciones no lineales, cuyas soluciones exhiben una rica variedad de comportamientos dinámicos. Esto incluye la posibilidad de movimiento caótico, incluso en sistemas de pocos grados de libertad, en los cuales las trayectorias se entremezclan entre sí de forma errática y turbulenta, de manera que resulta imposible toda predicción detallada para tiempos largos.

La existencia de caos y su complejidad intrínseca ya fué puesta de manifiesto a finales del siglo pasado, sobresaliendo la figura de Poincaré. Sin embargo el posterior desarrollo de esta teoría se vio frenado, debido en parte, a la dificultad intrínseca que supone la no integrabilidad de las ecuaciones que describen estos fenómenos. No fue hasta la década de los sesenta, con el desarrollo de los modernos ordenadores digitales y la publicación de una serie de resultados matemáticos importantes, cuando se pudo profundizar en aquellas ideas pioneras de la Dinámica No lineal.

La característica más importante del movimiento caótico es su extrema sensibilidad a pequeñas variaciones en las condiciones iniciales, de forma que dos trayectorias que empiezan muy próximas se separan de forma exponencial en el tiempo. Hay que destacar, que, a pesar de su naturaleza impredecible, el caos es un fenómeno determinista en el que la evolución del sistema viene dada en todo momento por un sistema de ecuaciones bien definido.

Otra característica importante del caos es su ubicuidad, habiéndose descrito fenómenos caóticos en campos tan diversos como Astronomía, Fluidodinámica, Meteorología, plasmas, circuitos electrónicos, vibraciones, dinámica de poblaciones y plagas, reacciones químicas oscilantes, etc. Esto ha hecho de la Dinámica No lineal un área tremendamente multidisciplinar, lo que también ha contribuido a su rápido desarrollo en los últimos años.

Dentro de la clasificación general de sistemas no integrables, tienen una importancia especial los sistemas conservativos, es decir aquellos para los que 
su energía total permanece constante. Existe una formulación de la dinámica de estos sistemas, debida a Hamilton, que permite su estudio sistemático

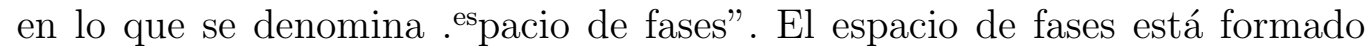
por las coordenadas generalizadas (coordenadas independientes que periten describir completamente el estado del sistema, también denominadas grados de libertad) y sus momentos conjugados, de forma que su dimensión es el doble del número de grados de libertad. Mientras que existen herramientas matemáticas bien definidas para visualizar y estudiar la estructura regular o caótica de sistemas de dos grados de libertad (que denominaremos sistemas 2D), como por ejemplo la superficie de sección de Poincaré, en el caso de 3 grados de libertad (sistemas $3 \mathrm{D}$ ), la situación es mucho más complicada y no está completamente resuelta. Esto es debido a que en este caso el espacio de fases tiene dimensión 6 o 5 si la energía total del sistema se conserva.

Denominamos indicador dinámico a cualquiera de estas herramientas matemáticas que nos permita la clasificación de la naturaleza (regular o caótica) de una trayectoria a partir de las ecuaciones del movimiento y de las condiciones iniciales. En la literatura científica aparecen bastantes indicadores, algunos de los cuales se remontan a los trabajos iniciales de Poincare, como las Superficies de Sección de Poincaré). Podemos clasificar a los indicadores en dos grandes grupos: por un lado, los indicadores que se basan exclusivamente en las características de la propia orbita investigada (como el mapa de frecuencias, por ejemplo) y por otro lado, los indicadores que analizan la órbita en función del comportamiento de órbitas cercanas, como, por ejemplo, el coeficiente de Lyapunov. El índice SALI (Small Alignment Index), que será usado de manera central en esta tesis, pertenece a este último tipo de indicadores. Este índice se basa en analizar cómo se separan trayectorias cercanas mediante un algoritmo muy eficiente que es independiente de la dimensionalidad del sistema.

Con el ánimo de desarrollar nuevas herramientas numéricas y de visualización que permitan profundizar en el conocimiento de las características del espacio de fases de los sistemas hamiltonianos multidimensionales no lineales en este trabajo analizamos la estructura del espacio de fases del sistema molecular LiNC/LiCN con dos y tres grados de libertad. Este sistema se puede considerar genérico, en contra posición a los hamiltonianos modelo empleados con cierta frecuencia en los trabajos sobre sistemas no integrables. Debido a las características del potencial 2D empleado, como por ejemplo su acusada anarmonicidad o la presencia de una barrera relativamente baja que separa los dos isómeros, en este sistema aparecen trayectorias caóticas a energías poco elevadas, lo que le hace especialmente interesante. Mientras que en el modelo 2D la distancia C-N se mantienen constante en su valor de equilibrio, la extensión al modelo 3D se realiza mediante un potencial Morse que 
describe la interacción entre los átomos de carbono y nitrógeno.

La forma en la que hemos abordado este estudio es mediante la obtención de diferentes mapas que asignan un valor de SALI a cada condición inicial de un determinado subconjunto del espacio de fases y en el diseño de visualizaciones multidimensionales para su representación gráfica.

Otro de los aspectos originales de esta tesis consiste en la utilización conjunta de diferentes indicadores dinámicos en la caracterización del espacio de fases del sistema. En el caso del sistema 2D se presentan Superficies de Sección de Poincaré con SALI y Diagramas de Bifurcación con SALI . En el caso del sistema 2D, se presentan visualizaciones 4D de los correspondientes mapas antes citados, así como análisis de frecuencias con SALI y energías cinéticas medias con SALI. Estos dos últimos mapas corresponden a una representación de la dinámica en el espacio de frecuencias y en el de energías respectivamente que permiten una completa caracterización de la dinámica y una correcta interpretación de la misma en sistemas de más de dos grados de libertad

La organización de esta tesis es la siguiente:

- Introducción teórica. En esta sección se exponen de mnera breve, desde el punto de vista de la Mecánica Clásica, los fundamentos del formalismo hamiltoniano y los indicadores de caos. Se definen las superficies de sección de Poincaré, el análisis de frecuencias y el índice SALI. Se describe extensamente el sistema molecular $\mathrm{LiNC} / \mathrm{LiCN}$ del que se aporta tanto su descripción general, la geometría del sistema y la descripción del movimiento, el hamiltoniano (distinguiendo los casos del potencial 2D y el modelo general en 3D). Se describen finalmente las ecuaciones del movimiento del sistema (ecuaciones de Hamilton) y las ecuaciones de su flujo tangente (euaciones de variación)

- Modelo 2D. Dedicamos la siguiente sección a a la definición de los mapas utilizados y la discusión de los resultados obtenidos con el modelo con dos grados de libertad con los mapas de SALI, las superficies de sección de Poincaré y la evolución temporal del indicador SALI. Acabamos nuestro estudio del modelo 2D discutiendo la relación entre el volumen de regularidad y caos y la energía vibracional del sistema.

- Modelo 3D En esta sección caracerizamos elespacio de fases correpsondeinte al sistema 3D. Se definende los distintos mapas que se utilizan y se estudia la forma en que varían las zonas de la regularidad y el caos del espacio de faes en función de energía. Por último comparamos los resultados obtenidos en los modelos de $2 \mathrm{D}$ y $3 \mathrm{D}$. 
- El sistema molecular $L i N C / L i C N$ a baja energía Por último empleamos las herramientas desarrolladas en la tesis para analizar el estado del sistema a un nivel muy bajo de energía. Se trata de una energía muy baja a la que no se espera encontrar órbitas caóticas lo que nos permite simplificar nuestro problema. La comparación entre los mapas del analisis de frecuencias con SALI y de las energías medias con SALI nos permite identificar diversas clases de grupos de órbitas cuyas características se detallan y se aportan ejemplos concretos de sus órbitas.

En el último capítulo se enumeran las conclusiones más relevantes de la tesis y posteriormente se incluye las referencias y varios apéndices explicativos. 
Parte I

TEORÍA 



\section{Capítulo 2}

\section{Sistemas Hamiltonianos}

En este capítulo presentamos brevemente la teoria físico-matemática que es la base de los principales conceptos que se exponen en esta tesis.

\subsection{Sistemas Hamiltonianos}

Para definir la posición de un sistema constituído por $\mathrm{N}$ partículas en el espacio, es necesario utilizar $3 N$ coordenadas.

El número de cantidades independientes que tienen que ser especificadas para definir unívocamente la posición del sistema dinámico, se denomina el número de grados de libertad del mismo. Estas cantidades no tienen que ser necesariamente las coordenadas cartesianas de las N partículas. De hecho muchas veces el elegir otras cantidades puede simplificar la resolución de un problema determinado.

Cualesquiera $N$ cantidades $q_{1}, q_{2}, \ldots, q_{N}$ que definan completamente la posición de un sistema con $N$ grados de libertad, se denominan coordenadas generalizadas del sistema.

A las derivadas temporales $\dot{q}_{i} \frac{d q_{i}}{d t}, \quad i=1,2, \ldots, N$ se denominan velocidades generalizadas [1].

\subsubsection{Formalismo de Lagrange}

Existen varias formulaciones equivalentes de las ecuaciones fundamentales de la mecánica clásica. Una de ellas es la de Lagrange, la cual caracteriza a un sistema dinámico por medio de una función denominada Lagrangiana $L(q, \dot{q}, t)$. 
De acuerdo con el formalismo lagrangiano, la segunda ley de Newton puede ser escrita en términos de la energía cinética $(T)$ de un cuerpo de masa $m$ y de la energía potencial $(V)$ asociada a la fuerza $\vec{F}$. La función lagrangiana es definida como:

$$
L=T-V
$$

La configuración del sistema viene determinada de manera unívoca en términos de las coordenadas generalizadas $q_{i}(t)$. En la elección de las coordenadas generalizadas se encuentra todo el sentido físico del análisis y una correcta elección proporcionará una buena solución de la mecánica del sistema. Las coordenadas generalizadas están relacionadas con las coordenadas cartesianas $x, y, z$ por relaciones del tipo:

$$
\begin{gathered}
x=x\left(q_{1}(t), \ldots, q_{N}(t), t\right), \\
y=y\left(q_{1}(t), \ldots, q_{N}(t), t\right), \\
z=z\left(q_{1}(t), \ldots, q_{N}(t), t\right)
\end{gathered}
$$

La energía cinética $T$ se puede expresar en función de las velocidades generalizadas $\dot{q}_{i}=\frac{d q_{i}}{d t}$ como:

$$
T=\frac{m}{2} \sum_{j} \sum_{k} A_{j k} \dot{q}_{j} \dot{q}_{k}
$$

donde

$$
A_{j k}=\frac{\partial x}{\partial q_{j}} \frac{\partial x}{\partial q_{k}}+\frac{\partial y}{\partial q_{j}} \frac{\partial y}{\partial q_{k}}+\frac{\partial z}{\partial q_{j}} \frac{\partial z}{\partial q_{k}}
$$

Siguiendo el formalismo de Lagrange, las ecuaciones del movimiento de un sistema con $N$ grados de libertad son un sistema de $N$ ecuaciones diferenciales ordinarias de segundo orden para las coordenadas generalizadas $q_{1}(t), \ldots, q_{N}(t)$. El movimiento del sistema está unívocamente determinado a partir de $2 N$ condiciones iniciales conocidas, es decir, las magnitudes de todos los $q_{i}$ y $\dot{q}_{i}$ en un tiempo particular $t_{0}$. El movimiento se puede representar geométricamente mediante una curva asociada al espacio de configuraciones, $\left(q_{1}, \ldots, q_{N}\right)$. Definiendo la Lagrangiana a partir de la ecuación 2.1, las ecuaciones del movimiento del sistema pueden ser escritas en la forma:

$$
\frac{\partial L}{\partial q_{i}}-\frac{d}{d t}\left(\frac{\partial L}{\partial \dot{q}_{i}}\right)=0 \quad i=1, \ldots N
$$




\subsubsection{Formalismo de Hamilton}

En el formalismo de Lagrange las coordenadas $q$ y las velocidades $\dot{q}$ se introducen de manera asimétrica (solo las $q_{i}$ son fundamentales mientras que $\dot{q}_{i}$ son variables derivada). Ademas obtenemos un sistema de ecuaciones diferenciales de segundo orden.

W.R Hamilton demostró que se puede conseguir un formalismo mucho más simétrico y elegante, sustituyendo las ecuaciones de Lagrange por un sistema equivalente de primer orden, con el doble del número de ecuaciones, describiendo la dinámica mediante las $2 N$ cantidades $q_{1}, \ldots, q_{N}, p_{1}, \ldots, p_{N}$, donde $p_{i}$ es el momento canónico conjugado de $q_{i}$ definido por:

$$
p_{i}=\frac{\partial L}{\partial \dot{q}_{i}}, i=1, N
$$

El formalismo Hamiltoniano sustituye las variables $(q, \dot{q})$ por $(q, p)$ e introduce la función $H(q, p, t)$ denominada Hamiltoniana en lugar de la Lagrangiana $L(q, \dot{q}, t)$ para analizar la dinámica. Este cambio de formalismo se hace mediante una transformación de Legendre, que en el presente contexto consiste en la sustitución de las velocidades generalizadas por los momentos canónicos como variables básicas y en la introducción de la función de Hamilton o Hamiltoniana $H(q, p, t)$ definida por:

$$
H(q, p, t)=\sum_{i=1}^{N} \dot{q}_{i} p_{i}-L(q, \dot{q}, t) .
$$

Las consecuencias más inmediatas de la introducción de la función $\mathrm{H}$ pueden ser deducidas diferenciando la ecuación 2.7, de manera que:

$$
d H=\sum_{i=1}^{N}\left(\dot{q}_{i} d p_{i}+p_{i} d \dot{q}_{i}\right)-\left[\sum_{i=1}^{N}\left(\frac{\partial L}{\partial q_{i}} d q_{i}+\frac{\partial L}{\partial \dot{q}_{i}} d \dot{q}_{i}+\frac{\partial L}{\partial t} d t\right)\right] .
$$

Teniendo en cuenta la definición 2.7 y las ecuaciones de Lagrange 2.5 se puede reducir la ecuación 2.8 a:

$$
d H=\sum_{i=1}^{N}\left(\dot{q}_{i} d p_{i}-\dot{p}_{i} d q_{i}\right)-\frac{\partial L}{\partial t} d t
$$

Por otro lado tenemos que

$$
d H=\sum_{i=1}^{N}\left(\frac{\partial H}{\partial q_{i}} d q_{i}+\frac{\partial H}{\partial p_{i}} d p_{i}\right)+\frac{\partial H}{\partial t} d t
$$


Así, comparando las ecuaciones 2.9 y 2.10 , resulta:

$$
\dot{q}_{i}=\frac{\partial H}{\partial p_{i}}, \dot{p}_{i}=-\frac{\partial H}{\partial q_{i}}, i=1, . ., N
$$

Además,

$$
\frac{\partial H}{\partial t}=-\frac{\partial L}{\partial t}
$$

Las ecuaciones 2.11 son las llamadas ecuaciones de Hamilton o ecuaciones canónicas de Hamilton y forman un conjunto de $2 \mathrm{~N}$ ecuaciones diferenciales de primer orden equivalente al sistema de $N$ ecuaciones de segundo orden de Lagrange. Las cantidades $(q, p)$ son llamadas variables canónicas y el espacio cartesiano de $2 N$ dimensiones cuando los puntos son representados por $(q, p) \equiv\left(q_{1}, \ldots, q_{N}, p_{1}, \ldots, p_{N}\right)$ es el llamado espacio de fase.

Un punto del espacio de fase define el estado del sistema en un determinado tiempo, de manera que el teorema de la existencia y unicidad garantiza que la especificación del estado del sistema en un tiempo $t_{0}$ determina una solución única $(q(t), p(t))$ para las ecuaciones de Hamilton. Así que, por cada punto del espacio de fase pasa una única trayectoria dinámica $(q(t), p(t))$, y dos trayectorias diferentes nunca se cruzan.

Otro resultado importante es que, si sabemos que la Lagrangiana usual de un sistema viene determinada por $L=T-V$ y se dan las siguientes condiciones:

- (i) T es una función exclusivamente cuadrática de las velocidades.

- (ii) V no depende de las velocidades.

Entonces, por el teorema de Euler de las funciones homogéneas, tenemos:

$$
\sum_{i} \dot{q}_{i} \frac{\partial L}{\partial \dot{q}_{i}}=\sum_{i} \dot{q}_{i} \frac{\partial T}{\partial \dot{q}_{i}}=2 T
$$

Como consecuencia, sustituyendo la ecuación 2.13 en la ecuación 2.7, la Hamiltoniana de un sistema viene dada por $H=2 T-T+V=E$, es decir, la Hamiltoniana es la energá total expresada en función de las coordenadas y momentos. Las condiciones (i) y (ii) prevalecen en la mayoría de los casos de interés físico, que incluye los sistemas que se estudian en esta tesis.

Estas condiciones sólo son suficientes, es decir, es posible que no se cumplan y aun así $H$ sea la energía total. Como ejemplo de sistema que satisface esta afirmación, tenemos el caso de una partícula en un campo electromagnético externo. 
Se puede demostrar que, en el caso de las fuerzas generalizadas que actúan en el sistema deriven de una función de Energía Potencial, la Hamiltoniana correspondiente será una constante del movimiento.

En efecto, para un sistema formado por un conjunto de partículas de masa $m_{i}$, tenemos:

$$
\frac{d H}{d t}=\sum_{i}\left[\frac{\partial H}{\partial q_{i}} \frac{d q_{i}}{d t}+\frac{\partial H}{\partial \dot{q}_{i}} \frac{d \dot{q}_{i}}{d t}\right] .
$$

Teniendo en cuenta que $T=T\left(\dot{q}_{i}\right)$ y que la fuerza que actúa sobre la partícula $i$ viene dada por $\vec{F}=m_{i} \ddot{\vec{q}}=-\vec{\nabla} V$ :

$$
\frac{d H}{d t}=\sum_{i}\left[\frac{\partial V}{\partial q_{i}} \dot{q}_{i}+m_{i} \dot{q}_{i} \ddot{q}_{i}\right]=0 .
$$

Es importante observar que de acuerdo con la ecuación 2.12, la Hamiltoniana dependerá explícitamente del tiempo si y sólo si lo mismo ocurre con la Lagrangiana $L$.

\subsubsection{Transformaciones Canónicas}

Una transformación de variables en el espacio de fases que es interesante es aquella en la que se preserva la forma canónica de las ecuaciones del movimiento. De manera más precisa, dadas las variables canónicas $(q, p)$, la Hamiltoniana $H(q, p, t)$ y las ecuaciones de Hamilton 2.11, se puede definir la transformación invertible:

$$
Q_{i}=Q_{i}(q, p, t), P_{i}=P_{i}(q, p, t), i=1, \ldots N
$$

de manera que sea posible encontrar una función $K(Q, P, t)$, donde las ecuaciones del movimiento para las nuevas variables tengan la forma Hamiltoniana:

$$
\dot{Q}_{i}=\frac{\partial K}{\partial P_{i}}, \dot{P}_{i}=-\frac{\partial K}{\partial Q_{i}}, i=1, . ., N
$$

Es importante destacar que las ecuaciones del movimiento en las nuevas variables deben tener la forma Hamiltoniana para cualquier función Hamiltoniana original. Así que, la validez simultánea de las ecuaciones 2.11 y 2.17, implica la validez simultánea de los principios variacionales:

$$
\delta \int_{t_{1}}^{t_{2}}\left[\sum_{i=1}^{N} p_{i} d \dot{q}_{i}-H(q, p, t)\right] d t=0 .
$$


$\mathrm{y}$

$$
\delta \int_{t_{1}}^{t_{2}}\left[\sum_{i=1}^{N} P_{i} d \dot{Q}_{i}-K(Q, P, t)\right] d t=0 .
$$

Una condición suficiente para la validez de 2.18 y 2.19 es que los respectivos integrandos difieran en la derivada total respecto el tiempo de una función $\Theta(q, p, t)$ ya que

$$
\delta \int_{t_{1}}^{t_{2}} \frac{d \Theta}{d t} d t=\delta \Theta\left(\left(q\left(t_{2}\right), p\left(t_{2}\right), t_{2}\right)-\delta \Theta\left(q\left(t_{1}\right), p\left(t_{1}\right), t_{1}\right)=0 .\right.
$$

La igualdad 2.20 se debe al hecho de que $\delta q_{i}\left(t_{1}\right)=\delta q_{i}\left(t_{2}\right)=0$ y $\delta p_{i}\left(t_{1}\right)=$ $\delta p_{i}\left(t_{2}\right)=0$. Así, la ecuación 2.16 preserva la forma Hamiltoniana de las ecuaciones del movimiento si:

$$
\sum_{i=1}^{N} p_{i} \dot{q}_{i}-H=\sum_{i=1}^{N} P_{i} \dot{Q}_{i}-K+\frac{d \Theta}{d t}
$$

o en la forma diferencial

$$
\sum_{i=1}^{N}\left(p_{i} d q_{i}-P_{i} d Q_{i}\right)+(K-H) d t=d \Theta
$$

Se puede concluir entonces, que la transformación 2.16 es canónica si y sólo si la ecuación 2.22 se cumple para alguna función $\Theta$. La forma $2.22 \mathrm{su}-$ giere evaluar $\Theta$ como una función de las coordenadas antiguas y nuevas. Así, podemos considerar por ejemplo $(q, Q)$, como un conjunto de $2 N$ variables independientes. Definiendo la función generadora $\Gamma_{1}(q, Q, t)[1]$ por:

$$
\Gamma_{1}(q, Q, t)=\Theta(q, p(q, Q, t), t) .
$$

y usando la ecuación 2.22, se comprueba que:

$$
p_{i}=\frac{\partial \Gamma_{1}}{\partial q_{i}}, \quad P_{i}=-\frac{\partial \Gamma_{1}}{\partial Q_{i}}
$$

y además.

$$
K(Q, P, t)=H(q, p, t)+\frac{\partial \Gamma_{1}}{\partial t}
$$

Por lo tanto, una vez definida la función $\Gamma_{1}(q, Q, t)$, una transformación canónica queda automáticamente definida por las ecuaciones 2.22, con la Hamiltoniana transformada dada por la ecuación 2.25. 
En determinados casos puede no ser conveniente considerar $(q, Q)$ como variables independientes. Sin embargo, si consideramos $(q, P)$ como $2 N$ variables independientes, habrá una función generadora del tipo $\Gamma_{2}(q, P, t)$, de manera que:

$$
\begin{gathered}
p_{i}=\frac{\partial \Gamma_{2}}{\partial q_{i}}, \quad q_{i}=\frac{\partial \Gamma_{2}}{\partial p_{i}}, \\
K(Q, P, t)=H(q, p, t)+\frac{\partial \Gamma_{2}}{\partial t} .
\end{gathered}
$$

De esta manera, definida la función $\Gamma_{2}(q, P, t)$, por la solución de las $N$ primeras ecuaciones 2.22 obtenemos $P_{i}=P_{i}(q, p, t)$; con este resultado, se determinan además $Q_{i}=Q_{i}(q, p, t)$ y la transformación obtenida es canónica.

Un resultado importante es el hecho de que la evolución de un sistema mecánico de un estado inicial $t$ a un estado en el tiempo $t+d t$, corresponde a la transformación:

$$
Q_{i}(t)=q_{i}(t+d t), P_{i}(t)=p_{i}(t+d t), i=1, \ldots N,
$$

que es una transformación canónica infinitesimal generada por la Hamiltoniana del sistema [1].

\subsubsection{Notación Simpléctica}

Es posible introducir una notación compacta que permite escribir todas las ecuaciones de Hamilton en una sola ecuación matricial. Sea $\mathbf{z}$ un vector con $2 N$ elementos, $z_{1}, \ldots, z_{2 N}$, definidos por:

$$
z_{i}=q_{i}, z_{N+i}=p_{i}, i=1, \ldots, N .
$$

De manera análoga, el vector $\partial H / \partial z$ es una columna con los siguientes elementos:

$$
\left(\frac{\partial H}{\partial z}\right)_{i}=\frac{\partial H}{\partial q_{i}},\left(\frac{\partial H}{\partial z}\right)_{N+i}=\frac{\partial H}{\partial p_{i}}, i=1, \ldots, N .
$$

Se define así mismo la matriz $\mathbf{J}$ de dimensiones $2 N \times 2 N$ :

$$
\mathbf{J}=\left(\begin{array}{cc}
\mathbf{0}_{N} & \mathbf{I}_{N} \\
-\mathbf{I}_{N} & \mathbf{0}_{N}
\end{array}\right)
$$

donde $\mathbf{I}$ es la matriz identidad.

Las ecuaciones de Hamilton en notación simpléctica vienen dadas por:

$$
\dot{\mathbf{z}}=\mathbf{J} \frac{\partial H}{\partial z} \text {. }
$$


Para el caso de una transformación canónica con las variables transformadas $Q=Q(q, p)$ y $P=P(q, p)$, que aquí representamos en la forma simpléctica por $\varsigma=\varsigma(z, t)$, la condición para que ésta sea canónica viene dada por:

$$
\mathbf{M}^{T} \mathbf{J M}=\mathbf{J}
$$

donde $\mathbf{M}$ es la matriz Jacobiana de la transformación $\varsigma=\varsigma(z, t)$ que viene dada por:

$$
\mathbf{M}=\left|\frac{\partial\left(\varsigma_{1}, \ldots, \varsigma_{2 N}\right)}{\partial\left(z_{1}, \ldots, z_{2 N}\right)}\right|
$$

Es fácil comprobar que, para una transformación canónica se cumple que $\operatorname{det}(\mathbf{M})= \pm 1[2]$

\subsubsection{El Teorema de Liouville}

Sea $\Sigma$ una región del espacio de fase $(q, p)$, que se transforma en la $\Sigma^{*}$ del espacio de fase $(Q, P)$ por una transformación canónica. Se puede definir el volumen $\Omega$ de la región $\Sigma$ como:

$$
\Omega=\int_{\Sigma} d q_{1} \ldots d q_{N} d p_{1} \ldots d p_{N} \equiv \int_{\Sigma} d^{2 N} z
$$

De manera análoga, el volumen en el espacio (Q,P) viene dado por:

$$
\Omega^{*}=\int_{\Sigma^{*}} d Q_{1} \ldots d Q_{N} d P_{1} \ldots d P_{N} \equiv \int_{\Sigma^{*}} d^{2 N} \varsigma
$$

Mediante el teorema del cambio de variables en una integral múltiple, tenemos:

$$
\Omega^{*}=\int_{\Sigma^{*}} d^{2 N} \varsigma=\int_{\Sigma}\left|\frac{\partial\left(\varsigma_{1}, \ldots, \varsigma_{2 N}\right)}{\partial\left(z_{1}, \ldots, z_{2 N}\right.}\right| d^{2 N} z .
$$

Como el determinante de la matriz Jacobiana de una transformación canónica es igual a \pm 1 , tenemos que

$$
\Omega^{*}=\int_{\Sigma^{*}} d^{2 n} \varsigma=\int_{\Sigma} d^{2 n} z=\Omega
$$

es decir, el volumen de cualquier región del espacio de fase es invariante bajo las transformaciones canónicas.

Por lo tanto, si $\Sigma_{0}$ es una región del espacio de fase en el tiempo $t_{0}$ con volumen $\Omega_{0}$ y $\Sigma_{t}$ es la región en el tiempo $t$ obtenida trasladando cada punto 
de $\Sigma_{0}$ en el punto correspondiente en $\Sigma_{t}$, a través de la evolución temporal generada por la Hamiltoniana, entonces:

$$
\Omega_{t}=\Omega_{0},
$$

pues la evolución temporal es una transformación canónica como hemos visto en la sección 2.1.3. Esta última ecuación es conocida como el Teorema de Liouville, que garantiza que la evolución de la dinámica mantiene el volumen de cualquier región del espacio de fase.

\subsubsection{Corchetes de Poisson}

El corchete de Poisson de dos funciones $F, G$ respecto a las variables canónicas $(p, q)$ está dado por:

$$
[F, G]=\sum_{i}\left(\frac{\partial F}{\partial q_{i}} \frac{\partial G}{\partial p_{i}}-\frac{\partial F}{\partial p_{i}} \frac{\partial G}{\partial q_{i}}\right) .
$$

Tiene las siguientes propiedades:

\section{Antisimetría:}

$$
[F, G]=-[G, F]
$$

\section{Bilinearidad:}

$$
[a F+b G, H]=a[F, H]+b[G, H]
$$

Identidad de Jacobi:

$$
[F,[G, H]]+[H,[F, G]]+[G,[H, F]]=0 .
$$

Supongamos que $F$ es una función del tiempo, así como de las coordenadas y los momentos generalizados. La derivada total de $\mathrm{F}$ con respecto al tiempo viene dada por:

$$
\frac{d F}{d t}=\sum_{i}\left(\frac{\partial F}{\partial q_{i}} \dot{q}_{i}+\frac{\partial F}{\partial p_{i}} \dot{p}_{i}\right)+\frac{\partial F}{\partial t} .
$$

Teniendo en cuenta las ecuaciones de Hamilton 2.11 y la definición del corchete de Poisson 2.40, obtenemos la siguiente expresión:

$$
\frac{d F}{d t}=[F, H]+\frac{\partial F}{\partial t} .
$$

donde $H$ es el Hamiltoniano del sistema. Cuando el corchete de Poisson de una función que no dependa explícitamente del tiempo se anula, se tendrá que 
$F$ será una constante durante todo el movimiento del sistema. La presencia de este tipo de cantidades está íntimamente relacionada con la integrabilidad o no de los sistemas dinámicos.

Debido a la propiedad 2.41, el corchete de Poisson de una función consigo misma es cero. Entonces, si hacemos que la función $F$ en 2.45 sea el Hamiltoniano $H$ y este no depende explícitamente del tiempo, se llega a la conservación de la energía.

En términos del corchete de Poisson, las ecuaciones de Hamilton 2.11 pueden escribirse de una forma elegante y simétrica:

$$
\dot{q}_{i}=\left[q_{i}, H\right], \dot{p}_{i}=\left[p_{i}, H\right] .
$$

\subsubsection{Sistemas Integrables}

Supóngase que se tiene un Hamiltoniano independiente del tiempo con $N$ grados de libertad. Si en este sistema es posible encontrar $N$ funciones $F_{i}(q, p), i=1,2 \ldots N$ independientes entre sí que permanecen constantes a lo largo de cada una de las trayectorias del sistema, se dice entonces que éste es integrable.

Cada una de las $F_{i}$ tiene un valor constante $\alpha_{i}$ a lo largo del movimiento, por lo que puede plantearse el sistema de ecuaciones:

$$
F_{i}(q, p)=\alpha_{i}, i=1,2 \ldots N
$$

que permite expresar $p$ como función de $q$ y del vector constante $\alpha$. Para que el sistema tenga solución, se requiere que, además de que las $N$ cantidades sean independientes, se cumpla la condición:

$$
\left[F_{i}, F_{j}\right]=0 \quad \forall i, j=1,2 \ldots N
$$

Cuando se cumple la condición 2.48 , se dice que las $F_{i}$ se encuentran en involución.

Al introducir la solución de 2.47, el Hamiltoniano será sólo función de las coordenadas (no de los momentos) y la resolución del sistema 2.11 se reduce al cálculo de $N$ integrales. Un sistema integrable por tanto, puede siempre reducirse a cuadraturas.

También podría elegirse a estas $N$ cantidades como los nuevos momentos mediante una transformación canónica de coordenadas $(q, p) \rightarrow(Q, F \equiv$ $P)$. Esto implica $\dot{P}_{i}=0$ para $i=1,2 \ldots N$. Por la condición anterior, este Hamiltoniano será función solamente de los nuevos momentos y no de las nuevas coordenadas ya que de otra forma no se cumpliría la segunda parte 
de las ecuaciones de Hamilton. La solución para las coordenadas es muy simple:

$$
Q_{i}=\beta_{i}(F) t+\delta_{i}
$$

donde $\beta$ depende solamente de los momentos $F$ y las $\delta_{i}$ son constantes de integración que se determinan a partir de las condiciones iniciales.

La existencia de estas $N$ constantes $F_{i}$ implica que una trayectoria determinada estará en una superficie $M$ de dimensión no mayor que $N$ dentro del espacio de fases $2 N$-dimensional. Para dilucidar la estructura geométrica de esta superficie, consideremos el siguiente campo vectorial en el espacio de fases:

$$
\mathbf{V}_{i}=\left(\nabla_{p} F_{i},-\nabla_{q} F_{i}\right), i=1,2 \ldots N
$$

Dado que las $F_{i}$ son independientes, también lo es cada uno de los vectores $\mathbf{V}_{i}$. Además, dado un punto de la superficie $M$, cualquier vector $\mathbf{V}_{j}$ será paralelo a la superficie en ese punto. Esto es así porque cada $\mathbf{V}_{j}$ es perpendicular a cada una de los vectores normales a $M$ :

$$
\mathbf{V}_{j} \cdot\left(\nabla_{p} F_{i},-\nabla_{q} F_{i}\right)=\left[F_{j}, F_{i}\right]=0
$$

debido a que las $F_{i}$ están en involución. Ahora bien, al restringirnos a un movimiento acotado, se tiene que $M$ es compacto. Entonces podemos usar un teorema de topología que establece que una variedad compacta paralelizable con $N$ campos vectoriales continuos y linealmente independientes, debe ser un toro $N$ dimensional. Con ello hemos encontrado la variedad en donde se lleva a cabo la dinámica de cualquier sistema integrable. A estas variedades se les llama toros invariantes debido a que una trayectoria que empieza en ella, permanece ahí todo el tiempo.

Las consideraciones anteriores se engloban en el Teorema de Arnol'dLiouville. que se enuncia a continuación:

\section{Teorema de Arnol'd Liouville}

- Sean $F_{1}, F_{2}, \ldots, F_{n} n$ integrales primeras independientes en involución de un un sistema Hamiltoniano.

- Sean $I_{c_{1}, c_{2}, \ldots, c_{n}}$ la variedad diferencial definida por las ecuaciones

$$
F_{i}=c_{i}
$$

donde $\left(c_{1}, c_{2}, \ldots, c_{n}\right)$ es un valor regular de la aplicación $F_{1} \times F_{2} \cdots \times$ $F_{n}: U \rightarrow \mathbf{R}^{n}$

entonces 
- $I_{c_{1}, c_{2}, \ldots, c_{n}}$ es invariante por el flujo. Es decir si una curva solución tiene un punto en la variedad $I_{c_{1}, c_{2}, \ldots, c_{n}}$ entonces está totalmente contenida en ella

- Cada componente conexa de $I_{c_{1}, c_{2}, \ldots, c_{n}}$ es difeomorfa a $\left.\mathbf{R}^{n} \times\left(S^{1}\right)^{(} n-k\right)$ con $0<k<n$

- El flujo sobre cada componente conexa de $I_{c_{1}, c_{2}, \ldots, c_{n}}$ está conjugado diferenciablemente con el flujo de translación sobre $\left.\mathbf{R}^{n} \times\left(S^{1}\right)^{(} n-k\right)$

Se puede encontrar una demostración detallada en [3]

Parece natural entonces encontrar un sistema de coordenadas apropiado para describir el movimiento en el toro. Dado que la elección de los nuevos momentos $F$ es arbitraria, uno puede escoger de la misma manera a $N$ cantidades independientes $I_{i}$, que sean funciones de $F_{i}, I_{i}=I_{i}(\mathbf{F})$.

A las $I_{i}$ se les denomina variables de acción cuando están definidas por las siguientes ecuaciones:

$$
I_{i}=\frac{1}{2 \pi} \oint_{\pi_{i}} \mathbf{p} d \mathbf{q} .
$$

Dado que un toro $N$ dimensional tiene exactamente $N$ circuitos irreductibles $\pi_{i}$ que se forman siguiendo en cada punto del toro la dirección marcada por cada uno de los vectores tangentes $\vec{V}_{i}$, cada una de las acciones $I_{i}$ se obtiene de calcular la integral a lo largo de cada uno de estos circuitos. Para calcular estas integrales, será necesario poner las $p_{i}$ en función de $q_{i}$ usando las ecuaciónes 2.47 .

A las coordenadas conjugadas correspondientes se les llama variables ángulo $\theta$. Al igual que antes, las acciones son constantes a lo largo de todo el movimiento, por tanto $H=H(I)$ únicamente. La solución de las ecuaciones de Hamilton en variables acción ángulo lleva a las siguientes expresiones:

$$
\begin{gathered}
\dot{I}_{i}=-\frac{\partial H(\mathbf{I})}{\partial \theta} \Rightarrow I_{i}(t)=I_{i}\left(t_{0}\right) \\
\dot{\theta}_{i}=\frac{\partial H(\mathbf{I})}{\partial I} \Rightarrow \theta_{i}(t)=\omega_{i} t+\theta_{i 0},
\end{gathered}
$$

donde las $\theta_{i 0}$ son constantes de integración que dependen de las condiciones iniciales y las $\omega_{i}$ son las frecuencias del movimiento a lo largo de cada uno de los circuitos en el toro y son solamente función de las acciones. 


\subsection{Sistemas de un grado de libertad}

Todo sistema Hamiltoniano de un grado de libertad que no dependa del tiempo por el hecho de que la energía se conserve es integrable pues tiene tantas integrales del movimiento (la energía) como grados de libertad.

Por ejemplo, en el caso de un sistema conservativo unidimensional compuesto de una sola partícula de masa $m$ moviéndose en el eje $Z$ bajo la acción de un potencial $V(z)$, el hecho de que la energía $E$ sea constante, permite escribir la solución de la ecuación del movimiento en la forma:

$$
\frac{1}{2} m\left(\frac{d z}{d t}\right)^{2}=(E-V(z)) \Rightarrow t-t_{0}=\sqrt{\frac{m}{2}} \int_{z_{0}}^{z} \frac{d z}{\sqrt{E-V(z)}} .
$$

Para conocer el comportamiento del sistema es necesario estudiar la estructura del espacio de fases $(q, p)$ y los tipos de movimiento que pueden presentarse. Por ejemplo, partiendo de las ecuaciones de Hamilton 2.11 con $N=1$, podemos observar que si el sistema se encuentra en un punto en el se cumple que $\partial H / \partial q=\partial H / \partial \mid q=0$, el sistema permanecerá ahí todo el tiempo. Estos puntos fijos pueden ser estables o inestables. Un punto será estable, si trayectorias inicialmente cercanas a él permanecen cercanas durante todo el tiempo. Por el contrario, un punto fijo será inestable si trayectorias inicialmente cercanas a él se alejan de él a medida que evolucionan en el tiempo.

Veamos cómo podemos aplicar la formulación Hamitoniana y las transformaciones canónicas para resolver la dinámica de dos sistemas típicos de un grado de libertad: el péndulo simple y el oscilador armónico.

\subsubsection{Péndulo simple}

El Hamiltoniano de un péndulo simple de masa $m$ y longitud $l$ como el representado en el panel izquierdo de la figura 2.1 viene dado por

$$
H(p, \theta)=m g l(1-\cos \theta)+p^{2} /\left(2 m l^{2}\right) .
$$

El espacio de fases viene representado por el momento conjugado a la coordenada $\theta$ angular $P_{\theta}=m \dot{\theta}$ en el eje de las ordenadas y el ángulo $\theta$ en el eje de las abscisas, tal y como se muestra en la parte derecha de la figura 2.1

En este caso se tiene un punto fijo estable en $\theta=0$. Las trayectorias cercanas corresponden a elipses en torno a este punto. Por esta razón, los puntos fijos estables son también llamados puntos elípticos.

También existe un punto fijo inestable correspondiente a $\theta=\pi$. En este caso, trayectorias inicialmente cercanas se alejarán de él. A los puntos fijos 

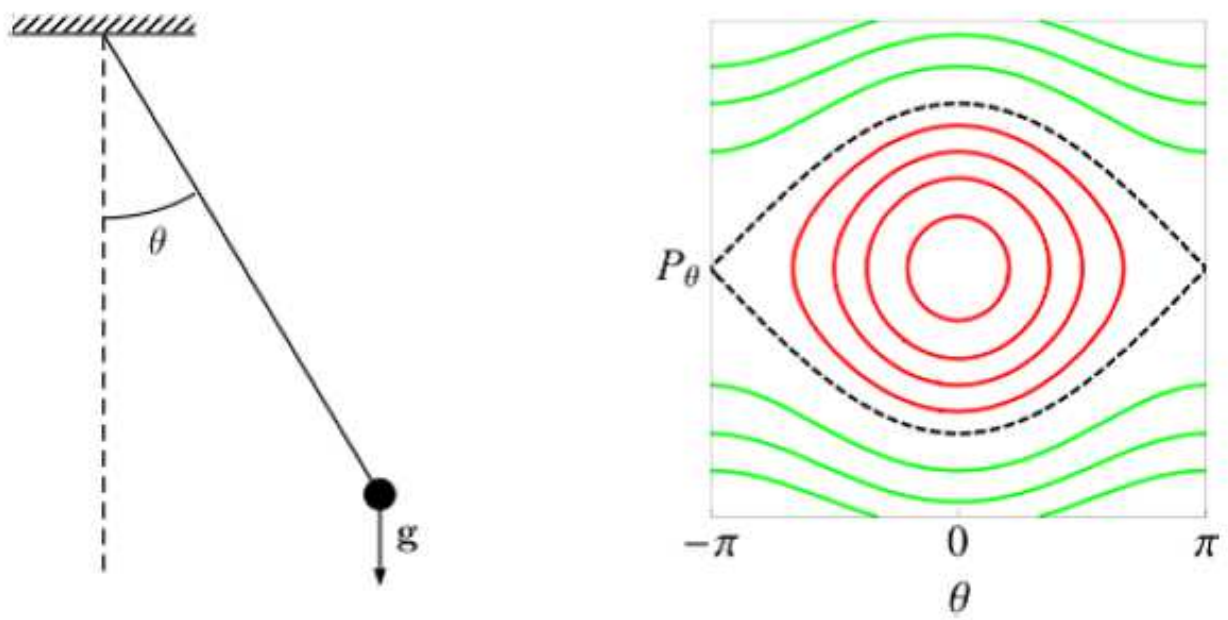

Figura 2.1: Izquierda: Péndulo Simple. Derecha: Espacio de Fases correspondiente, en rojo los movimientos de libración y en verde los de rotación, la linea de trazos negra es la separatriz

inestables también se les llama puntos hiperbólicos. La curva que pasa por el punto hiperbólico corresponde a una separatriz del movimiento. En el espacio de fases la zona que se encuentra dentro de la separatriz presenta un movimiento periódico, también llamado libración. Fuera de la separatriz se tendrán movimientos de rotación.

\subsubsection{Oscilador armónico}

En esta sección vamos a usar una transformación canónica de coordenadas para resolver el problema del oscilador armónico unidimensional, cuyo Hamiltoniano viene dado por:

$$
H=\frac{p^{2}}{2 m}+\frac{m \omega^{2} q^{2}}{2} .
$$

Para ello vamos a realizar el siguiente cambio de variables:

$$
P=\frac{1}{b^{2}}\left(p^{2}+a^{2} q^{2}\right)
$$

donde $a$ y $b$ son constantes. Ahora precisamos encontrar un cambio de coordenadas $Q=Q(q, p)$ de manera que, junto a la ecuación 2.59 conforme una transformación canónica. Si reescribimos 2.59 como:

$$
p=b\left(P-c^{2} q^{2}\right)^{1 / 2}, c=\frac{a}{b}
$$


se puede comprobar que, para encontrar la transformación canónica resulta adecuado la búsqueda de una función generadora del tipo $\Gamma_{2}(q, P)$. Por lo tanto:

$$
p=\frac{\partial \Gamma_{2}}{\partial q}=b\left(P-c^{2} q^{2}\right)^{1 / 2} \Rightarrow \Gamma_{2}=b \int\left(P-c^{2} q^{2}\right)^{1 / 2} d q
$$

La otra mitad de la transformación canónica que viene dada por:

$$
Q=\frac{\partial \Gamma_{2}}{\partial P}=\frac{b}{2} \int \frac{d q}{\left(P-c^{2} q^{2}\right)^{1 / 2}}=\frac{b^{2}}{2 a} \sin ^{-1}\left(\frac{a q}{b \sqrt{P}}\right) .
$$

La transformación inversa viene dada por:

$$
q=\frac{b}{a} \sqrt{P} \sin \left(\frac{2 a Q}{b^{2}}\right), p=b \sqrt{P} \cos \left(\frac{2 a Q}{b^{2}}\right) .
$$

Teniendo en cuenta la forma del Hamiltoniano 2.58 y las transformaciones 2.63 elegimos $a=m \omega$ y $b=\sqrt{2 a}$. Como la función generatriz $\Gamma_{2}(q, P)$ no depende del tiempo tenemos que:

$$
\Theta(Q, P)=H(q, p)=\frac{p^{2}}{2 m}+\frac{m \omega^{2} q^{2}}{2}=\frac{\omega}{2 m \omega}\left(p^{2}+m^{2} \omega^{2} q^{2}\right)=\omega P
$$

de forma que:

$$
\begin{gathered}
\dot{Q}=\frac{\partial \Theta}{\partial P}=\omega \Rightarrow Q=\omega t+\delta \\
\dot{P}=-\frac{\partial \Theta}{\partial Q}=0 \Rightarrow P=\frac{E}{\omega}=c t e .
\end{gathered}
$$

donde $E$ es la energía total del oscilador. Finalmente, teniendo en cuenta 2.63 obtenemos la solución del movimiento del oscilador, que viene dada por:

$$
\begin{aligned}
& q(t)=\sqrt{\frac{2 E}{m \omega^{2}}} \sin (\omega t+\delta) \\
& p(t)=\sqrt{2 m E} \cos (\omega t+\delta)
\end{aligned}
$$

\subsection{Sistemas de dos o más grados de libertad}

\subsubsection{Sistemas integrables de dos grados o más grados de libertad}

Consideremos el caso de un sistema integrable con dos o más grados de libertad. Dado que el sistema es integrable, entonces debe existir una segunda 
(a)

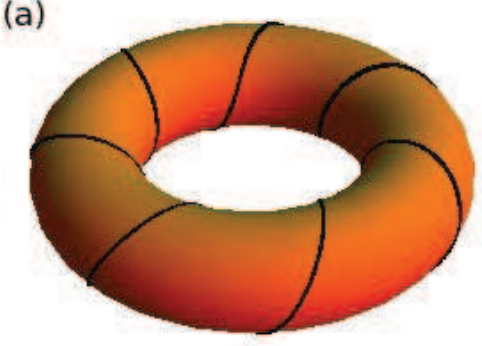

(b)

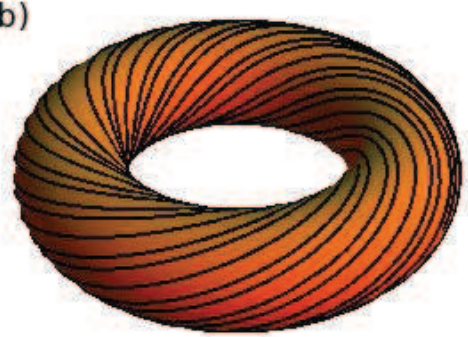

Figura 2.2: Trayectorias dentro de un toro para una relación de frecuencias $\omega_{1} / \omega_{2}$ (a) racional y (b) irracional

cantidad conservada, además de la Energía, por lo que las trayectorias en el espacio fases se moverán a lo largo de un toro de dimensión 2.

La frecuencia $\omega_{1}$, se mide con respecto a uno de los circuitos en el toro y la frecuencia $\omega_{2}$, con respecto al otro. Si la relación entre las frecuencias $\omega_{1} / \omega_{2}$ es un número racional, la trayectoria volverá a encontrarse en el mismo punto después de un cierto número de vueltas, y será por lo tanto cerrada. Se dice entonces que el sistema presenta una resonancia. A este tipo de trayectorias se les conoce como órbitas periódicas. La representación de dicha trayectoria en una superficie de sección de Poincaré (que se describe en detalle en el capitulo 3, pero que ahora podemos considerar como un corte de la trayectoria con un plano en el espacio de fases) nos dará un número finito de puntos. Por otro lado, si la relación de frecuencias es un número irracional, la trayectoria cubre densamente la superficie del toro. Se tiene entonces una órbita cuasiperiódica. La proyección de estas trayectorias en una superficie de sección corresponderá a curvas cerradas. En la figura 2.2 se muestran estos dos tipos de dinámica.

Para el caso de $N$ dimensiones, las características básicas del movimiento en dos dimensiones se mantienen, sin embargo, para tener órbitas periódicas deberán tenerse $N-1$ relaciones del tipo:

$$
\omega \cdot \mathbf{m}=0
$$

donde $\omega$ es el vector de frecuencias dado en la ecuación 2.55 y $\mathbf{m}$ es un vector de números enteros. En caso de no tener estas relaciones, el movimiento será cuasiperiódico y la trayectoria cubrirá densamente la superficie del toro $N$ dimensional. 


\subsubsection{Sistemas no integrables. Caos}

En general, un sistema con dos ó más grados de libertad no es separable, ya que habrá acoplamiento entre los grados. Por lo tanto estos sistemas no son en general integrables. El hecho de que un sistema sea no integrable, implica que no hay una solución analítica para las ecuaciones del movimiento en términos de las condiciones iniciales y del tiempo.

Añadir acoplamientos a sistemas que son inicialmente separables, lleva a cambios cualitativos muy importantes en el comportamiento de las trayectorias. En particular estos acoplamientos llevan al caos determinista, fenómeno asociado a las altas inestabilidades de las soluciones del sistema [4].

La representación de las trayectorias de un sistema de dos grados de libertad en el espacio de fases es muy complicado ya que se trata de un espacio de dimensión 4. Pero, como vamos a estudiar sistemas conservativos en los que la energía, $E$, se conserva, resulta que el movimiento se produce en una región de dimensión 3, llamada hipersuperficie de energía, de forma que el vínculo $H(q, P)=E$ debe cumplirse.

En el caso de no existir tantas integrales de movimiento como grados de libertad, no es posible reducir el sistema a variables acción ángulo. Para estudiar la dinámica en estos casos, partimos de un Hamiltoniano integrable y posteriormente lo perturbamos ligeramente añadiendo un término no integrable. El nuevo Hamiltoniano estará dado por:

$$
H(I, \theta)=H_{0}(I)+\epsilon H_{1}(I, \theta)
$$

donde consideraremos que $\epsilon$ es pequeño. $H_{0}$ es la parte integrable del Hamiltoniano y $H_{1}$ la no integrable. En este caso, el par $(I, \theta)$ continúa formado por parejas de variables canónicas. Sin embargo, no son ya variables acción ángulo ya que $\theta$ aparece en el Hamiltoniano $H$.

En este caso solamente tenemos una integral de movimiento (la energía), por lo cual las trayectorias estarán en la superficie tridimensional $H=$ constante. El comportamiento que se observa para perturbaciones pequeñas es que la mayor parte de la estructura que se tiene en el caso de sistemas integrables se mantiene, la mayoría de los toros invariantes se conservan, aunque deformados $[5,6,7]$.

Sin embargo, podemos encotrar trayectorias en donde el movimiento ya no estará confinado a la superficie del toro, sino que estará esparcido por la hipersuperficie $E=$ constante de manera caótica. En el caso de dos grados de libertad, la representación de estas trayectorias en la superficie de sección de Poincaré, corresponderá a un conjunto de puntos esparcidos a lo largo de la superficie de sección y la zona regular y la zona caótica estarán separadas. Las 
fronteras entre estas zonas corresponden a los últimos toros destruídos por la perturbación y éstos forman conjuntos de Cantor y son llamados Cantoros.

Si dos trayectorias se encuentran en la zona de movimiento integrable a lo largo de toros invariantes, entonces se tendrá que, si sus condiciones iniciales están dadas por dos puntos muy cercanos entre sí en el espacio de fases, al irse propagando en el tiempo, la distancia entre dos puntos a un mismo tiempo $t$ aumentará de manera lineal con respecto a la que se tenía inicialmente. Por otro lado, en el movimiento caótico esto se dará de manera exponencial, convirtiendo al movimiento en impredecible para todo fin práctico, dado que no será posible determinar las condiciones iniciales con una precisión infinita. Ésta gran sensibilidad a la elección de las condiciones iniciales, es de hecho, la característica fundamental de una trayectoria caótica.

\subsubsection{Teorema KAM}

Por lo que se ha visto en la sección anterior, es importante determinar los toros que se destruirán y los que sobrevivirán ante la presencia de una perturbación. El teorema de Kolmgórov, Arnold y Moser (Teorema KAM) $[8,9,10,11]$, resuelve esta cuestión. Para ello, parte de la expresión 2.70 e intenta encontrar, aplicando teoría de perturbaciones, una transformación canónica que dé lugar a un nuevo Hamiltoniano integrable que sólo dependa de las nuevas acciones. Se llega entonces a una expresión para la función generadora $S$ (que es de tipo 2 segń la nomenclatura de [1], denominada $\Gamma_{2}$ en la sección 2.1.3) que viene dado por un desarrollo en serie de la forma:

$$
S(\theta, J)=\theta \cdot J+\epsilon \sum_{\mathbf{m} \neq 0} \frac{\mathbf{A}_{\mathbf{m}}(I)}{\omega_{0} \cdot \mathbf{m}} \exp (i \mathbf{m} \cdot \theta)+O\left(\epsilon^{2}\right)
$$

donde las $\mathbf{A}_{\mathbf{m}}(I)$ son coeficientes de Fourier que dependen de las acciones $I$ y las frecuencias $\omega_{0}$ son las correspondientes al Hamiltoniano integrable.

El problema radica en estudiar la convergencia de esta serie. Se encuentra entonces que, para toros que se encuentren cerca de una resonancia (donde el término $\omega_{0} \cdot \mathbf{m}$ se anula), se tendrán divisores pequeños que ocasionarán la no convergencia de la serie dada en la ecuación 2.71. Informalmente, el teorema KAM establece que solo sobreviven los toros cuya relación de frecuencias $\omega_{i}$ $\omega_{j}$ sea lo suficientemente irracional.

Para determinar el grado de irracionalidad de un número partimos de la expresión en fracción continua de un número $\sigma$, que viene dada por

$$
\sigma=a_{0}+\frac{1}{a_{1}+\frac{1}{a_{2}+\frac{1}{a_{3}+\ldots}}}
$$


donde la secuencia $a_{0}, a_{1} a_{2} \ldots$ está conformada por números enteros. Esta secuencia es única para cada número y representa el procedimiento que converge más rápidamente posible al intentar encontrar una aproximación racional para un número irracional. Sea $\sigma_{n}$ una aproximación racional a $\sigma$ que utiliza $n$ números naturales para calcular su fracción continua, es decir:

$$
\sigma_{n}=a_{0}+\frac{1}{a_{1}+\frac{1}{a_{2}+\frac{1}{\ddots \cdot \frac{1}{a_{n}}}}}=\frac{r_{n}}{s_{n}}
$$

Esta secuencia siempre converge a $\sigma$. Además, es la mejor aproximación posible, en el sentido de que ningún racional $r / s$ con $s \leq s_{n}$ está más cerca de $\sigma$ que $r_{n} / s_{n}$. También se cumple la desigualdad:

$$
\left|\sigma-\frac{r_{n}}{s_{n}}\right|<\frac{1}{s_{n} s_{n-1}}
$$

La fracción continua de $\sigma$ converge más rápidamente, a medida que la sucesión $a_{0}, a_{1} \ldots$ diverge con mayor celeridad. La convergencia más lenta posible, la cual corresponde al número más irracional de todos, corresponde a la llamada proporción aurea $\gamma$ :

$$
\gamma=1+\frac{1}{1+\frac{1}{1+\frac{1}{1+\ldots}}}=\frac{\sqrt{5}-1}{2}
$$

Como ya hemos dicho el teorema KAM establece que sobrevivirán los toros cuya relación de frecuencias es lo suficientemente irracional, debiendo cumplirse la relación:

$$
|\mathbf{m} \cdot \omega|>k(\epsilon)|\mathbf{m}|^{-N-1}
$$

donde $\mathbf{m}$ es un vector de números enteros, $N$ es el número de grados de libertad del sistema y $k(\epsilon)$ es una cantidad que sólo depende de $\epsilon$ y que tiende a cero cuando la perturbación lo hace. Los toros que no cumplen esta condición, serán destruidos, aunque sobrevivirán un cierto número de puntos fijos, que darán lugar a la existencia de nuevos toros invariantes.

El Teorema KAM demuestra que la mayor parte de los toros invariantes del Hamiltoniano $H_{0}$ se deforman en presencia de la perturbación $\varepsilon H_{1}$, cuando el parámetro $\varepsilon$ es lo suficientemente pequeño. A medida que aumenta la perturbación los toros que cumplen $|\mathbf{m} \omega|=0$, donde $\omega$ es el vector con las frecuencias de los toros, se van rompiendo en función del valor de $\mathbf{m}$, siendo los últimos en romperse los que tienen un vector $\mathbf{m}$ mas irracional. En esa situación, las trayectorias dejan de estar confinadas, y pueden barrer libremente todo el espacio de fases accesible a la energía del sistema. Estas últimas trayectorias son extremadamente sensibles a las condiciones iniciales, y es a ellas a las que se denomina caóticas. 


\subsection{Dinámica linealizada}

En esta sección se estudian las ecuaciones lonealizadas que gobiernan el movimiento de las trayectorias próximas a una órbita de referencia, lo que nos permite estudiar la estabilidad de las órbitas periódicas así como la estructura del espacio de fases cerca de la misma.

Consideremos una trayectoria de referencia en el espacio de fase:

$$
z \overrightarrow{(t)}=\left(q_{1}(t), \ldots, q_{N}(t), p_{1}(t), \ldots \ldots, p_{N}(t)\right)
$$

y sea $\vec{z}_{1}(t)$ una trayectoria próxima.

Definimos el vector de desviación $\vec{v}_{1}$ como:

$$
\overrightarrow{v_{1}}(t)=\vec{z}(t)-\overrightarrow{z_{1}}(t)
$$

Las trayectorias $\vec{z}(t)$ y $\vec{z}_{1}(t)$ se derivan de las ecuaciones del movimiento a partir del Hamiltoniano:

$$
\begin{gathered}
\frac{d \vec{z}(t)}{d t}=J \nabla H(\vec{z}(t)) \\
\frac{d \vec{z}_{1}(t)}{d t}=J \nabla H\left(\vec{z}_{1}(t)\right)
\end{gathered}
$$

donde la matriz $J$ está dada por

$$
J=\left[\begin{array}{cc}
0_{N} & I_{N} \\
-I_{N} & 0_{N}
\end{array}\right]
$$

Combinando la (2.77) y la (2.79), tenemos:

$$
\frac{d\left(\vec{z}(t)-\vec{v}_{1}(t)\right)}{d t}=J \nabla H\left(\vec{z}(t)-\vec{v}_{1}(t)\right)
$$

Asumiendo que la función hamiltoniana $H$ es analítica y que la norma del vector de desviación $\left|\vec{v}_{1}\right|$ es pequeña, $H$ puede ser expandido en serie de Taylor hasta el primer orden, y

$$
H\left(\vec{z}(t)-\vec{v}_{1}(t)\right)=H(\vec{z}(t))-\nabla H(\vec{z}(t)) \vec{v}_{1}(t)
$$

Reemplazando Eq. (2.82) en Eq. (2.81) y teniendo en cuenta que $J$ y $\nabla$ son operadores lineales:

$$
\frac{d \vec{z}(t)}{d t}-\frac{d \vec{v}_{1}(t)}{d t}=J \nabla H(\vec{z}(t))-J \nabla^{2} H(\vec{z}(t)) \vec{v}_{1}
$$


Tomando la Eq. (2.78) en cuenta, podemos derivar la siguiente ecuación, que denominamos Ecuación de Variación

$$
\frac{d \vec{v}_{1}(t)}{d t}=J \nabla^{2} H(\vec{z}(t)) \vec{v}_{1}(t)
$$

Resolviendo de forma simultanea las ecuaciones (2.78) y (2.84) para un conjunto dado de condiciones iniciales $\left(z_{0}, v_{1}^{0}\right)$, podemos obtener tanto la trayectoria de referencia como los valores asociados al vector de desviación de la órbita cercana.

La matriz $M(t) \equiv J \nabla^{2} H(\vec{z}(t))$ recibe el nombre de matriz jacobiana. Se puede demostrar que se trata de una matriz simpléctica [2].

La matriz jacobiana $M(t) \equiv M(T)$ calculada al tomar un recorrido cerrado en el espacio de fases, es decir, tomando como trayectoria de referencia una órbita periódica y como tiempo $t$ el periodo $T$ de la misma se la define como matriz de monodromía o matriz de estabilidad. Permite caracterizar la estabilidad de las órbitas periódicas.

\subsection{Estabilidad de las órbitas periódicas}

El estudio de la estabilidad de una órbita periódica de un sistema se realiza analizando el comportamiento de las trayectorias próximas a la misma a través de los autovalores de la matriz de monodromía.

Consideremos una órbita periódica $\pi$ en un sistema con dos grados de libertad (2.1) y utilicemos un sistema de referencia en coordenadas curvilíneas según el cual la coordenada $x$ es la longitud desde el punto de partida hasta la posición actual y el eje $y$ es un eje perpendicular a la órbita de referencia que nos da la distancia que hay entre la trayectoria cercana y la órbita de referencia, que está definida por $y=0$.

Para un determinado punto de $\pi$, restringimos el estudio al movimiento en el plano transversal, definido por un valor constante de $x$ y $p_{x}$. Los puntos en este plano se caracterizan por la distancia $y$ a la órbita periódica y por el momento en la dirección normal a la misma, $p_{y}$, y quedan especificados por la matriz jacobiana del movimiento transversal $\mathbf{M}(t)$. Si la órbita de referencia es periódica, los elementos de la matriz jacobiana al cabo de un periodo $T$ $\mathbf{M} \equiv \mathbf{M}(T)$ dependen de las condiciones iniciales $\left(y_{0}, p_{y 0}\right)$ de partida, pero sus autovalores no. La matriz de estabilidad transversal $\mathbf{M}$ se puede escribir como:

$$
\mathbf{M} \equiv \mathbf{M}(T)=\left(\begin{array}{ll}
m_{11} & m_{12} \\
m_{21} & m_{22}
\end{array}\right)
$$


cuando el polinomio característico es:

$$
\eta_{ \pm}^{2}-\eta_{ \pm} \tau+\Delta=0
$$

donde $\tau$ representa la traza de la matriz, $\Delta$ su determinante, que es igual a la unidad por ser $\mathbf{M}$ una matriz simpléctica, y $\eta_{ \pm}$sus autovalores. Despejando $\eta_{ \pm}$de (2.86) resulta

$$
\eta_{ \pm}=\frac{1}{2}\left(\tau \pm \sqrt{\tau^{2}-4}\right)
$$

Las órbitas periódicas se clasifican atendiendo al valor que toman los autovalores de $\mathbf{M}$ :

- Órbita estable o elíptica. Los autovalores son complejos $(|\tau|<2)$

$$
\eta_{ \pm}=e^{ \pm i \omega T}
$$

donde $T$ es el periodo de la órbita.

- Órbita marginal. Los autovalores valen $\eta_{ \pm}= \pm 1$, dado que $\tau= \pm 2$.

- Órbita inestable . Los autovalores son reales debido a que $|\tau|>2$. En este caso hay a su vez dos tipos de OPs:

- Hiperbólica. Los dos autovalores son positivos $(\tau>2)$ y de la forma

$$
\eta_{+}=\eta, \quad \eta_{-}=\frac{1}{\eta}
$$

con $\eta>0$.

- Hiperbólica con reflexión. Los autovalores de la matriz de monodromía tienen la forma de $(2.89)$, pero con $\eta<0(\tau<-2)$.

Si los autovalores son reales, se suelen reescribir como

$$
\eta_{ \pm}= \pm \exp ( \pm \lambda T)
$$

donde $\lambda$ es el llamado coeficiente de estabilidad de la órbita, y el signo que precede a la exponencial es "+" si la órbita es hiperbólica y "_" si es hiperbólica con reflexión. El coeficiente de estabilidad se calcula despejándolo de la ecuación (2.89) y viene dado por

$$
\lambda=\frac{\ln |\eta|}{T}
$$

En régimen caótico todas las órbitas periódicas son de tipo inestables, hipérbolicas o hiperbólicas con reflexión. 


\section{Capítulo 3}

\section{Indicadores del caos}

La naturaleza caótica de las trayectorias de un sistema se puede estudiar por medio de ciertos indicadores. En este apartado describimos algunos de los indicadores más empleados en la literatura y que se utilizaran en esta tesis: la Superficie de Sección de Poincaré, los exponentes de Lyapunov (LCE), mapa de frecuencias y el índice SALI (Small Alignment Index).

\subsection{Superficie de Sección de Poincaré}

La Superficie de Sección de Poincaré (SSP) reduce el estudio de un sistema continuo al estudio de un mapa, y resulta especialmente útil para el estudio de sistemas Hamiltonianos de dos grados de libertad.

El espacio de fases de un Hamiltoniano de dos grados de libertad $H\left(p_{1}, p_{2}, q_{1}, q_{2}\right)$ tiene dimensión cuatro. Cuando el Hamiltoniano es conservativo, la energía $E$ es una constante del movimiento:

$$
H\left(q_{1}, q_{2}, p_{1}, p_{2}\right)=E .
$$

por lo que todas las trayectorias que son una solución de las ecuaciones del movimiento 2.30 se desarrollan en la hipersuperficie de dimensión tres definida por la ecuación 3.1. Entonces, conocido el valor de las coordenadas $\left(q_{1}, q_{2}\right)$ y el momento $p_{1}$ podemos calcular el del momento $p_{2}$ despejándolo de dicha ecuación

$$
p_{2}=p_{2}\left(q_{1}, q_{2}, p_{1} ; E\right) .
$$

La SSP más sencilla que se puede definir es aquélla que viene dada por el plano $q_{2}=q_{20}$ y tomando $p_{2}>0$. Entonces, cada vez que una trayectoria corte dicho plano con $p_{2}>0$, mapeamos el valor de $q_{1}$ y $p_{1}$. Si elegimos una SSP adecuada, como la representada en la figura 3.1, la representación de 

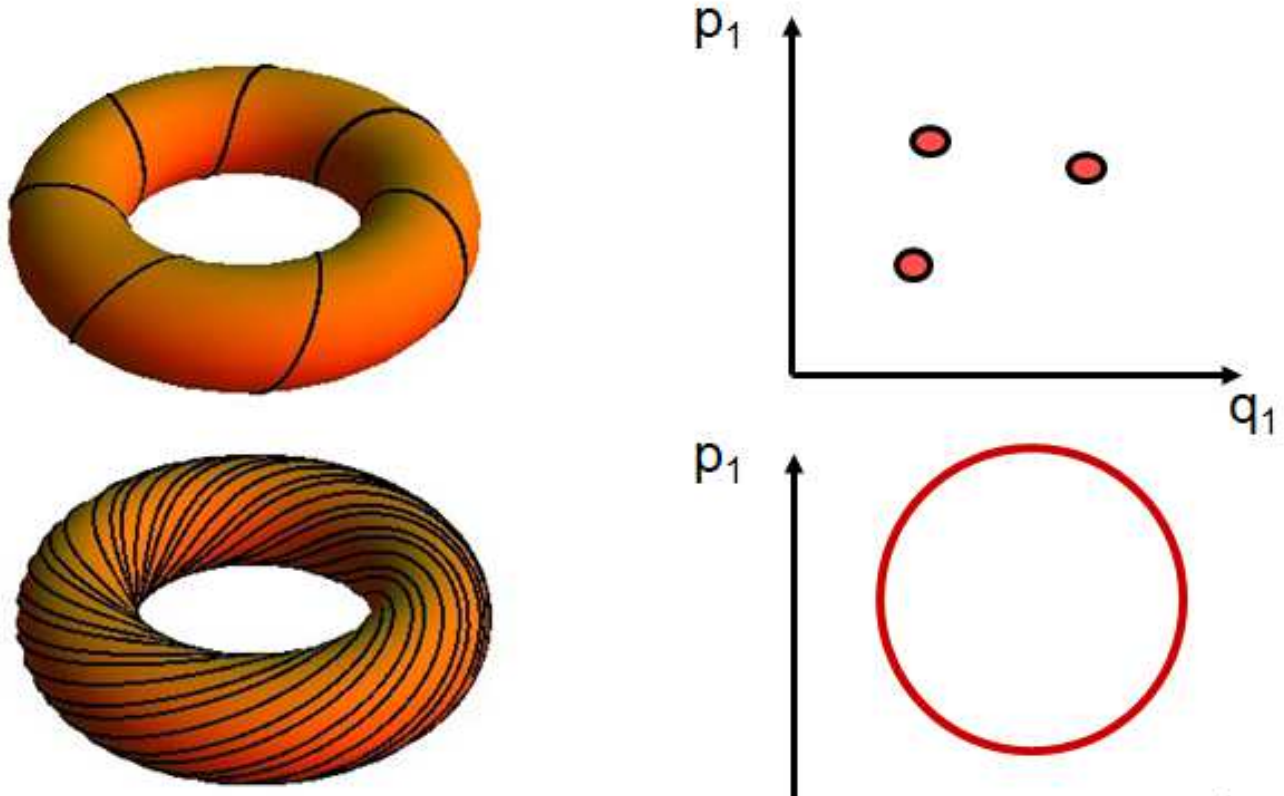

$\mathrm{p}_{1}$
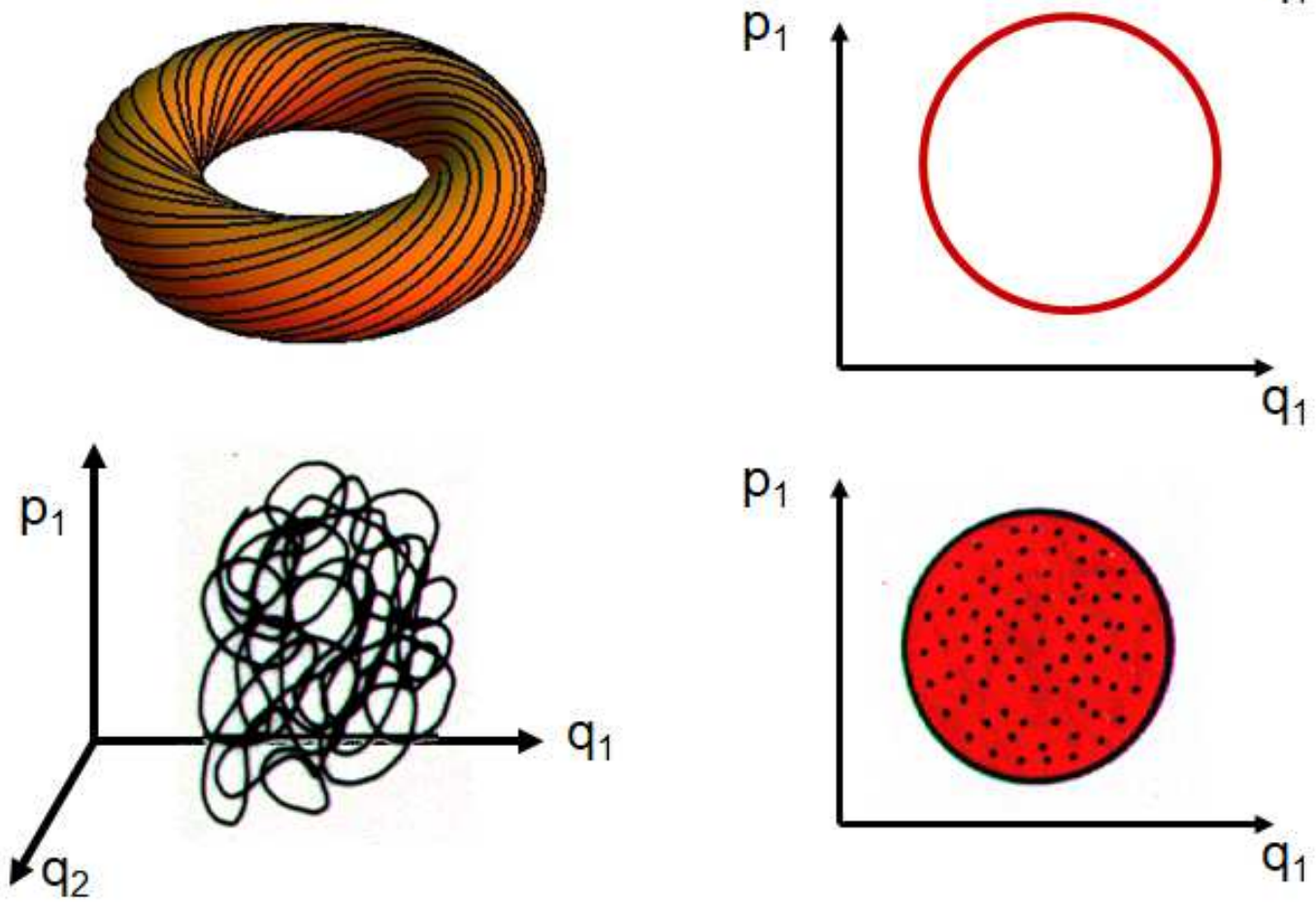

Figura 3.1: Trayectorias (izquierda) y sus SSP correspondientes (derecha). Las trayectorias son Periodicas (arriba), Cuasiperiodicas (medio) y Caóticas (abajo)

$\left(q_{1}, p_{1}\right)$ a lo largo del tiempo es suficiente para analizar el comportamiento de un sistema de dos grados de libertad. Así, las trayectorias caóticas se representan en la SSP como un conjunto denso de puntos, mientras que las trayectorias regulares, que están confinadas en los toros invariantes, se representan por medio de puntos discretos (órbitas periódicas) o de líneas continuas (órbitas cuasiperiódicas). Ver la figura 3.1

También podemos definir SSPs más complejas, en las que $q_{2}=q_{2}\left(q_{1}\right)$. En 
este caso, es necesario llevar a cabo una transformación canónica para que se satisfaga el Teorema de Liouville, de forma que el área mapeada por la SSP se conserve.

\subsection{Exponentes de Lyapunov (LCE)}

Los exponentes de Lyapunov miden la tasa de divergencia exponencial de órbitas inicialmente cercanas en el espacio de fase para un tiempo lo suficientemente grande [12]. Su existencia y unicidad están garantizados por el teorema de Osedelets [13].

De manera análoga a la que hicimos en la sección 2.4 estudiaremos ahora la evolución de una trayectoria $z^{\prime}(t)$, relacionada con la OP de referencia $z(t)$ por la ecuación

$$
\vec{z}^{\prime}(t)=\vec{z}(t)+\vec{v}(t)
$$

con la condición inicial

$$
\vec{z}_{0}^{\prime}=\vec{z}_{0}+\vec{v}_{0}
$$

En general, podemos estudiar también la evolución del vector $\vec{v}(t)$ que separa dos trayectorias cualesquiera como las mostradas en la figura 3.2 con tal de que esten lo suficientemente próximas, sin necesidad de que una de ellas sea periódica. Entonces, resolviendo las ecuaciones de movimiento (2.30), podemos definir la distancia entre dos puntos de la trayectoria como la norma euclídea

$$
d\left[\vec{z}_{0}, t\right]=\|\delta \vec{z}(t)\|=\left\|\vec{z}^{\prime}(t)-\vec{z}(t)\right\|
$$

y medir con ella la sensibilidad a las condiciones iniciales de una trayectoria cercana a la trayectoria de referencia $\mathbf{z}(t)$ por medio de los exponentes de Lyapunov, que se definen como

$$
\bar{\lambda}\left[\vec{z}_{0}, \delta \vec{z}_{0}\right]=\lim _{\substack{t \rightarrow \infty \\ d\left[z_{0}, 0\right] \rightarrow 0}} \frac{1}{t} \log \left(\frac{d\left[\vec{z}_{0}, t\right]}{d\left[\vec{z}_{0}, 0\right]}\right) .
$$

Al igual que hicimos en la sección 2.4 , cuando $d\left[\vec{z}_{0}, 0\right] \rightarrow 0$ podemos linealizar el movimiento y definir una base de vectores $\left\{\vec{\xi}_{i}\right\}_{i=1}^{n}$. Se puede demostrar entonces que existen $n$ exponentes de Lyapunov distintos $\bar{\lambda}_{i}=$ $\bar{\lambda}\left[\vec{z}_{0}, \vec{\xi}_{i}\right]$, asociados uno a cada dirección $\vec{\xi}_{i}$. Si $\bar{\lambda}_{i}>0$, la trayectoria $\mathbf{z}^{\prime}(t)$ se aleja exponencialmente de la trayectoria de referencia $\vec{z}(t)$ en esa dirección, mientras que si $\bar{\lambda}_{i}<0$, se acerca exponencialmente, al igual que sucede con los índices de estabilidad de las órbitas periódicas; de hecho, es habitual en la literatura referirse a ellos como exponentes de Lyapunov.

Se suele denominar exponente de Lyapunov de un sistema a 


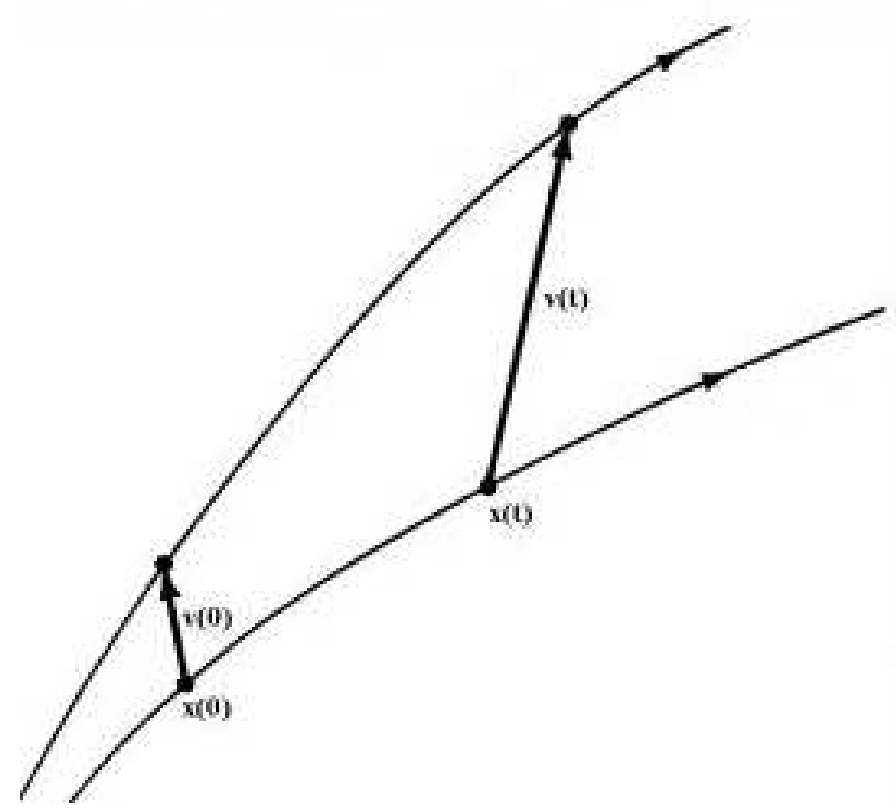

Figura 3.2: Evolución en el espacio de fases de dos trayectorias cercanas.

$$
\lambda^{\prime}=\operatorname{máx}\left\{\bar{\lambda}\left[\vec{z}_{0}, \delta \vec{z}_{0}\right], \forall \vec{z}_{0}\right\} .
$$

Un sistema se denomina caótico cuando $\bar{\lambda}>0$.

\subsection{Análisis de Frecuencias}

El análisis del mapa de frecuencias es un método basado en el análisis de Fourier, que facilita una representación de la dinámica de sistemas multidimensionales, mediante el paso del dominio del tiempo al dominio de la frecuencia.

La idea general de construir un mapa de frecuencias para estudiar el espacio de fases de un sistema Hamiltoniano se basa en el teorema KAM.

De acuerdo con el teorema KAM, descrito en la sección 2.3.3, en el espacio de fases de un sistema que esté lo suficientemente cercano a uno integrable, persisten muchos toros invariantes, es decir, toros en los que las trayectorias que comienzan en ellos permanecen, ejecutando movimientos cuasi periódicos, de acuerdo con un vector de frecuencias fijas que depende solo del toro.

Podemos calcular numéricamente, para un intervalo de tiempo finito, un 
vector de frecuencias para cualquier condición inicial (aunque estrictamente hablando, este tipo de vectores solo están definidos sobre toros). Este vector de frecuencias será una aproximación bastante buena de las frecuencias reales, si estamos ante un toro KAM.

El método más directo para encontrar el vector de frecuencias es utilizar el análisis de Fourier de una trayectoria integrada numéricamente. La descomposición de Fourier de una órbita cuasiperiodica nos da un espectro discreto de frecuencias. Naturalmente, la forma precisa de este espectro depende de las coordenadas que estemos usando, pero existen una serie de propiedades que son invariantes, como por ejemplo, las $\mathrm{N}$ frecuencias fundamentales a partir de las cuales se generan las líneas del espectro.

La precisión de la transformada de Fourier es del orden de $O\left(\ln \frac{2 \pi}{T}\right)$, donde $T$ es el tiempo de integración. Esta precisión se puede aumentar usando una base no ortonormal de frecuencias en el desarrollo de la serie de Fourier.

Jacques Laskar [14, 15] desarrolló esta idea creando una serie de herramientas numéricas (NAFF) que aplicó al estudio del movimiento débilmente caótico del sistema solar.

El método númerico parte de una trayectoria $[q(t), p(t)]$ en el espacio de fases y a partir de ella construye una serie temporal en el espacio complejo dada por :

$$
f(t)=q(t)+i p t
$$

El algoritmo del análisis de frecuencias nos dará la siguiente expresión de la función en forma de una serie:

$$
\vec{f}(t)=\sum_{i} a_{i} e^{i w_{i} t}
$$

con $\left|a_{1}\right|>\left|a_{2}\right|>\ldots$

La primera frecuencia, que denominamos frecuencia fundamental, viene dada por el término que se obtiene buscando el máximo de la función:

$$
\phi(\sigma)=\left\langle f(t), e^{i \sigma y}\right\rangle
$$

Donde el producto escalar $<f(t), g(t)>$ viene definido por la siguiente ecuación.

$$
<f(t), g(t)>=\frac{1}{2 T} \int f(t) g \overline{(t)} \xi\left(\frac{t}{T}\right) d t
$$

$\xi(t)$ es una función de peso, definida positiva y uniforme, tal que:

$$
\frac{1}{2} \int \xi(t) d t=1
$$


En esta tesis se usará como función de peso la siguiente expresión (denominada ventana de Hanning):

$$
\xi(t)=1+\cos (\pi t)
$$

Una vez calculada la primera frecuencia $\omega_{1}$ se obtiene su amplitud compleja $a_{1}$ mediante una proyección ortogonal de $f(t)$ sobre $e^{i \omega_{1} t}$ y el proceso se empieza de nuevo con la parte remanente de la función

$$
f_{1}(t)=f(t)-a_{1} e^{i \omega_{1} t}
$$

La función remanente obtenida $f_{1}(t)$ no es necesariamente ortogonal a $f(t)$. Así que se ortogonaliza $f_{1}(t)$ mediante el método de Granm-Schmid y se sigue con el procedimiento iterativamente hasta obtener la precisión deseada.

Si la función $f(t)$ es una solución cuasiperiódica o periódica del sistema Hamiltoniano, la serie 3.9 converge, y la frecuencia fundamental $\omega_{1}$ está asociada a la solución de las ecuaciones de Hamilton 2.55 y 2.56.

En este caso, cuando el sistema es regular, existe un difeomorfismo continuo entre las condiciones iniciales en el espacio de fases $\left(q_{i}, p_{i}\right)$ y las frecuencias asociadas a la trayectoria, en el espacio de frecuencias $w_{i}$ [16].

Cuando el sistema deja de ser regular, deja de existir este difeomorfismo entre el espacio de fases y el de frecuencias, de forma que la asociación de frecuencias a unas condiciones iniciales es arbitraria, dependiendo, por ejemplo, del periodo $\mathrm{T}$ de integración, rompiéndose la continuidad en la variación de las frecuencias cuando se varia infinitesimalmente las condiciones iniciales. De esta forma, variando de manera continua un parámetro del sistema o las condiciones iniciales en el espacio de fases, la ruptura de la continuidad en la variación de las frecuencias en el espacio de frecuencias, se puede utilizar como un indicador de caos muy efectivo.

Numéricamente se ha observado [17] que además el mapa de frecuencias es útil para detectar cadenas de islas, pues la zona del espacio de fases donde se encuentran los toros regulares produce una zona de valores casi constantes de las frecuencias.

En función de los grados de libertad del sistema, la representación del mapa de frecuencias puede cambiar. Usualmente, en sistemas con dos grados de libertad se suele representar las relación de las dos frecuencias $\omega_{1} / \omega_{2}$ en función de una de las condiciones iniciales. En la figura 3.3 se muestra un análisis de frecuencias típico, aplicado al sistema LiCN de dos grados de libertad, que se analiza en profundidad en el capitulo 5 En sistemas de tres grados de libertad se suele representar la relación $\left(\omega_{1} / \omega_{2}, \omega_{3} / \omega_{2}\right)$ para un conjunto de condiciones iniciales. En el capítulo 5 se presentan estos mapas 
aplicados al sistema LiCN con tres grados de libertad para distintas energías de excitación del sistema. El mapa de frecuencias se puede utilizar como indicador dinámico de la naturaleza regular o caótica de una órbita tanto en sistemas de dos grados de libertad [16], como en sistemas con tres grados de libertad [18].

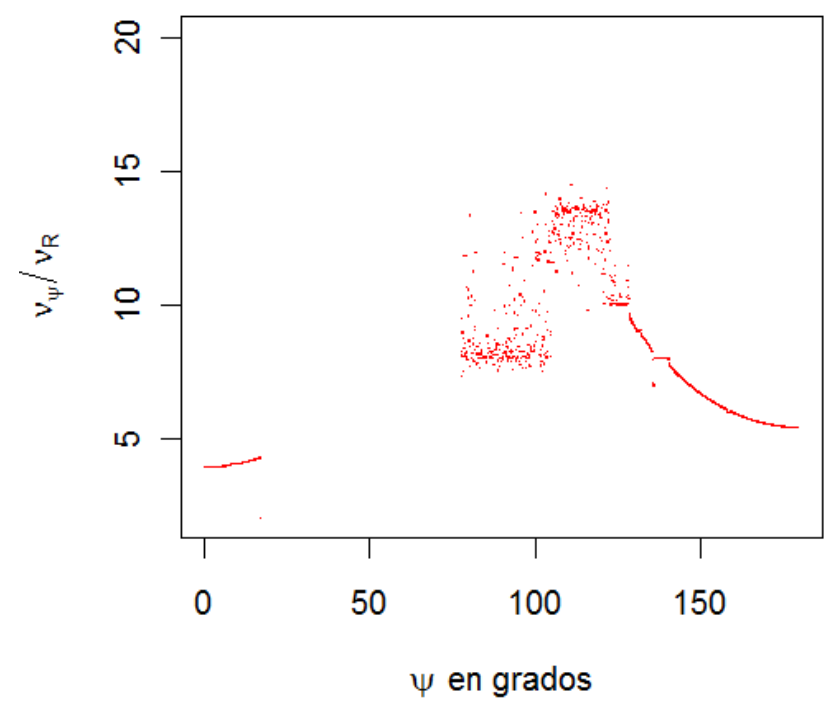

Figura 3.3: Mapa de frecuencias del LiCN/LiNC 2D a la energía $2549.2 \mathrm{~cm}^{-1}$. Se representa la relación entre las frecuencias de $\mathrm{R}$ y de $\psi$ en función del ángulo $\Psi$ 


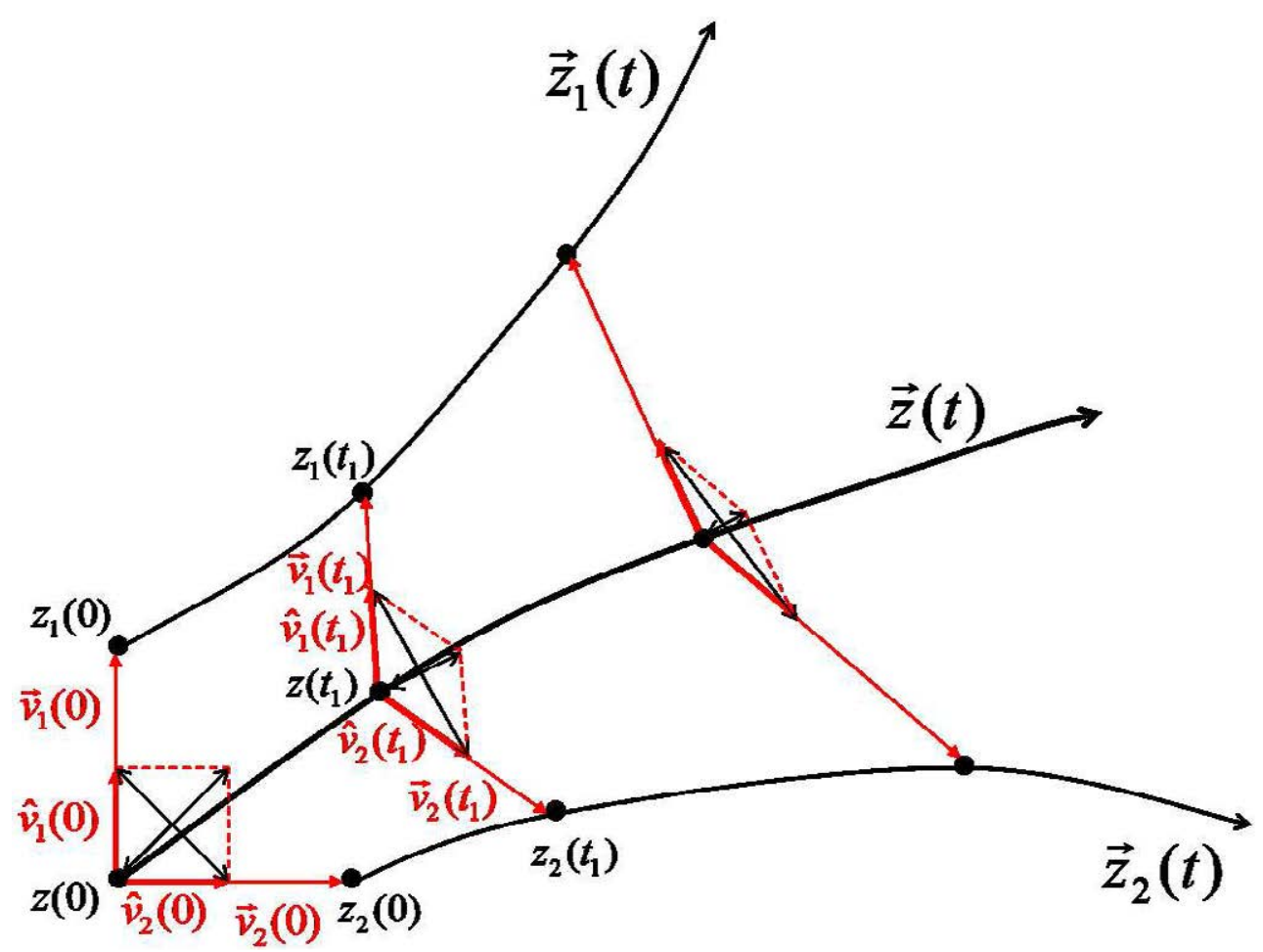

Figura 3.4: Ilustración grafica sobre la forma de la suma y la diferencia de dos vectores cercanos a la trayectoria de referencia, $\vec{z}(t)$. Aplicación al calculo de SALI

\subsection{Small Allignement Indicator (SALI)}

SALI (Small ALignement Index) es un indicador de cáos para flujos Hamiltonianos y mapas simplecticos propuesto por el astrónomo griego Ch. Skokos y su grupo [19, 20, 21, 24, 22] y que han aplicado con éxito al estudio de numerosos sistemas hamiltonianos multidimensionales [23, 24, 25, 26, 27].

Para una trayectoria dada, SALI estudia el comportamiento de los vectores asociados a la diferencia entre la trayectoria de referencia y trayectorias cercanas (vectores de desviación).

Desde un punto de vista intuitivo, la idea base de SALI es comprobar si los vectores de desviación entre la órbita en estudio y dos orbitas de su entorno se transforman (o no) en paralelos o anti paralelos.

Este último hecho nos indicaría que estamos ante una órbita caótica dado que se observa que la dirección de los vectores de desviación tiende a coincidir con la dirección de la variedad inestable más cercana en el caso de una trayectoria caótica. Si la órbita fuese regular los vectores de desviación acabarían por ser tangentes a un toro. En este caso SALI no tiende a cero sino que fluctúúa alrededor de un valor positivo. Estas consideraciones pue- 
den ser usadas para describir las características dinámicas de trayectorias de sistemas hamiltonianos genéricos independientemente de su dimensión y en particular en sistemas moleculares realistas [28, 29].

$\mathrm{El}$ procedimiento cuyo esquema se muestra en la figura 3.4 es el siguiente:

Consideremos la trayectoria de referencia $z \overrightarrow{(t)}=\left(q_{1}(t), \ldots, q_{N}(t), p_{1}(t), \ldots, p_{N}(t)\right)$ en el espacio de fase, sean $\vec{z}_{1}(t), \vec{z}_{2}(t)$ dos trayectorias próximas.

Definimos los vectores de desviación $\vec{v}_{i}$ como:

$$
\overrightarrow{v_{i}}(t)=\vec{z}(t)-\overrightarrow{z_{i}}(t) \quad i=1,2
$$

De acuerdo con la sección 2.4 los vectores de desviación $\tilde{\mathbf{v}}_{\mathbf{i}}$ siguen la ecuación de variación 2.14:

$$
\frac{d \vec{v}_{i}(t)}{d t}=J \nabla^{2} H(\vec{z}(t)) \vec{v}_{i}(t) \quad i=1,2
$$

Definiendo los vectores unitarios de desviación como:

$$
\hat{v}_{i}(t)=\frac{\overrightarrow{v_{i}}(t)}{\left|\overrightarrow{v_{i}}(t)\right|}, \quad i=1,2
$$

Resolviendo de forma simultanea la Eq. (2.78) y la Eq. (3.16) para un conjunto dado de condiciones iniciales $\left(z_{0}, v_{1,2}^{0}\right)$, obtenemos tanto la trayectoria de referencia como los valores asociados a los vectores de desviación de dos orbitas cercanas.

Se define el indicador SALI [19] como:

$$
S A L I\left(z_{0}, t\right)=\operatorname{mín}\left(\left|\hat{v}_{1}(t)+\hat{v}_{2}(t)\right|,\left|\hat{v}_{1}(t)-\hat{v}_{2}(t)\right|\right)
$$

Se ha demostrado $[19,20]$ que en una trayectoria caótica $\operatorname{SALI}\left(z_{0}, t\right) \sim$ $e^{-\lambda t}, \lambda>0, \lambda=\lambda_{1}-\lambda_{2}$, donde $\lambda_{1}, \lambda_{2}$ son los dos primeros coeficientes de Lyapunov [20] mientras que para una trayectoria regular, el valor de esta indicador oscila alrededor de un valor constante $S A L I\left(z_{0}, t\right) \sim \mathcal{O}(1)$. En el apéndice $\mathrm{B}$ se presenta una demostración geométrica de este comportamiento asintótico de SALI. En la figura 3.5 se muestra un ejemplo numérico de la validez de la aproximación asintótica del decaimiento exponencial para una órbita caótica de un Hamiltoniano con un potencial cúbico. 

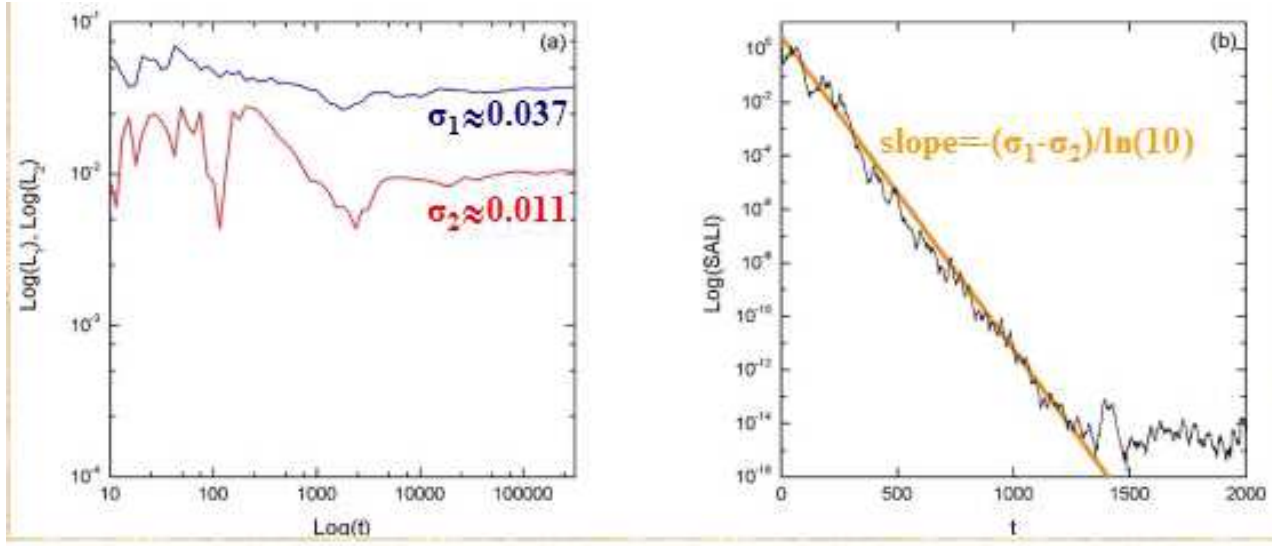

Figura 3.5: Test de la validez de la aproximación $\operatorname{sali}(t)=e^{-\lambda t}$ En la izquierda primeros coeficientes de Lyapunov $\sigma_{1}, \sigma_{2}$ de una órbita asociada al Hamiltoniano $H=\sum_{i=1}^{3} \frac{1}{2}\left(q_{i}^{2}+\right.$ $\left.\left.p_{i}^{2}\right)+q_{1}^{2} q_{2}+q^{2} q_{3}\right)$. A la derecha representación de $-\log \left(\operatorname{sali}(t)\right.$ y de la recta $s=-\frac{\left(\sigma_{1}-\sigma_{2}\right) t}{\ln ()}+$ $C$ en función del tiempo 


\section{Capítulo 4}

\section{Sistema Molecular LiNC/LiCN}

\subsection{Descripción General}

Los estudios que se presentan en esta tesis han sido realizados sobre el sistema molecular triaómico $\mathrm{LiNC} / \mathrm{LiCN}$. Este sistema presenta dos isómeros estables en las configuraciones lineales: $\mathrm{Li}-N C$ (isocianuro de litio) y $L i-C N$ (cianuro de litio). El primer isómero es el más estable y ambos están separados por una modesta barrera de energía de solo $3470 \mathrm{~cm}^{-1}$.

El movimiento en la coordenada de flexión de enlace es muy libre y esto hace que el átomo de litio pueda moverse con cierta facilidad en el entorno del fragmento $C \equiv N$, recorriendo amplias zonas de la superficie de energía potencial, donde la anarmonicidad y el acoplamiento entre modos es apreciable, ocasionando que el caos aparezca a valores relativamente bajos de la energía.

Por otro lado la frecuencia vibracional del $C N$ es muy alta, por lo que se puede considerar, en una primera aproximación, que se trata de un movimiento independiente de los otros modos vibracionales del sistema. Así, aprovechando la rigidez del enlace formado por los átomos de carbono y nitrógeno, podemos considerar además del caso general de tres grados de libertad, la aproximación de dos grados de libertad.

Todo esto hace que el sistema molecular $\operatorname{LiNC} / \operatorname{LiCN}$ sea un sistema muy interesante en física molecular, dado que puede ser descrito de manera relativamente realista, pudiéndose usar como banco de pruebas para caracterizar el comportamiento dinámico de sistemas hamiltonianos realistas de 2 y 3 grados de libertad.

A lo largo de este Capítulo, se definen las coordenadas más adecuadas para describir la superficie de energía potencial que se utilizará en el estudio de la dinámica vibracional de este sistema. 


\subsection{Geometría del sistema y descripción de su movimiento}

El sistema molecular $\mathrm{LiNC} / \mathrm{LiCN}$ está compuesto por tres átomos (litio, Li, carbono, $\mathrm{C}$, y nitrógeno, $\mathrm{N}$ ), presentando dos tipos de enlace; uno de tipo covalente y fuerte entre los átomos de carbono y nitrógeno $(C-N)$ y otro de tipo iónico, mucho más débil, entre el átomo de litio $(L i)$ y el grupo de $C-N$. Un sistema como éste, posee tres grados de libertad asociados al movimiento de cada uno de los núcleos, lo que se traduce en un total de nueve grados de libertad; tres de ellos asociados al movimiento traslacional, tres al vibracional y tres al rotacional.

El interés del estudio que se presenta en este trabajo, se centra en la dinámica vibracional del sistema, es decir en los movimientos vibracionales que conllevan la variación de las distancias relativas entre los núcleos atómicos. Considerando el sistema aislado de fuerzas externas, su movimiento traslacional correspondiente a las tres direcciones del espacio es separable y no afecta a la dinámica interna de éste. El movimiento rotacional no es, sin embargo, separable del vibracional. Afortunadamente, para estados no excitados rotacionalmente, es posible prescindir de las componentes rotacionales del potencial. Siguiendo el trabajo de Tennyson y Sutcliffe [30], el hamiltoniano para un sistema molecular triatómico, puede expresarse, mediante una transformación adecuada de coordenadas, en:

$$
H\left(x_{1} \ldots, x_{9}\right)=H_{t}\left(X_{1}, X_{2}, X_{3}\right)+H_{v r}\left(x_{1} \ldots, x_{6}\right)
$$

donde $H_{t}\left(X_{1}, X_{2}, X_{3}\right)$ corresponde al hamiltoniano del centro de masas del sistema y $H_{v r}\left(x_{1} \ldots, x_{6}\right)$ al hamiltoniano vibrorotacional. Esta expresión representa de manera separada el movimiento traslacional del centro de masas y el de vibración-rotación. Para separar $H_{v r}$ los autores proponen un nuevo cambio de coordenadas, tomando como coordenadas internas las de Jacobi $(R, r, \theta)$, que se describirán en la siguiente sección del presente capítulo (ver Figura 4.1)y como coordenadas de rotación, las de Euler $(\phi, \chi, \xi)$ de manera que $H_{v r}$ se puede expresar como:

$$
H_{v r}(R, r, \theta, \phi, \chi, \xi)=H_{v}(R, r, \theta)+H_{v r}^{\prime}(R, r, \theta, \phi, \chi, \xi)
$$

verificándose que, para el estado rotacional fundamental, $H_{v r}^{\prime}(R, r, \theta, \phi, \chi, \xi)=$ 0 .

Por otra parte, el fuerte enlace entre los núcleos atómicos de carbono y nitrógeno, provoca que, de forma aproximada, el movimiento relativo de estos dos átomos, pueda considerarse desacoplado del resto del sistema. De este 
modo, la distancia entre ellos puede considerarse fija e igual a la distancia de equilibrio, lo que reduce a dos el número de grados de libertad del sistema, permitiendo estudiar un modelo de 2D.

Esta aproximación y las anteriores, permiten estudiar la dinámica del sistema, considerando únicamente dos grados de libertad, asociados a la vibración del núcleo Li respecto al grupo $C=N$, donde el vector que describe la distancia $C-N, r$, permanece constante y las coordenadas utilizadas son $R$, distancia entre el átomo Li y el centro de masas del fragmento $C N$ y el $\theta$ el ángulo formado por estos dos vectores (ver figura 4.1).

También hay que tener en cuenta que, aunque las moléculas son entes cuánticos se puede abordar su estudio utilizando la mecánica clásica si tenemos en cuenta la aporximación de Born-Oppenheimer, que permite desacoplar los movimientos nucleares y electrónicos debido a la diferencia de masa entre ellos.

En esta tesis abordaremos el estudio de los movimientos vibracionales del sistema molecular LiNC/LiCN tanto mediante la aproximación de un sistema hamiltoniano 2D como mediante el sistema completo 3D.

\section{Unidades empleadas}

Las magnitudes utilizadas en el presente trabajo poseen escala molecular, por lo que se tomarán como unidades de masa y de longitud las correspondientes al sistema de unidades atómicas. En este sistema de unidades, la unidad atómica de masa se corresponde con la masa del electrón $m_{e}$ y la unidad de longitud con el radio de Bohr $a_{0}$, que representa el radio del átomo de hidrógeno en su estado fundamental.

A pesar de que la unidad atómica de energía y la utilizada en los cálculos es la del estado fundamental del átomo de hidrógeno, a lo largo de esta memoria se utilizará como unidad de energía la ampliamente empleada unidad espectroscópica $\mathrm{cm}^{-1}$. El cambio de unidaddes viene dado por la siguiente expresión:

$$
E\left(\mathrm{~cm}^{-1}\right)=219474,624 \cdot E(\text { u.a. })
$$

Por último, en las expresiones y valores numéricos se empleará como unidad angular el radian $(r a d)$.

\subsection{Descripción del Hamiltoniano}

Considerando el caso de momento angular total igual a cero, el Hamiltoniano vibracional correspondiente al sistema molecular $\mathrm{LiNC} / \mathrm{LiCN}$ en las coordenadas de Jacobi (ver figura 4.1) viene dado por: 


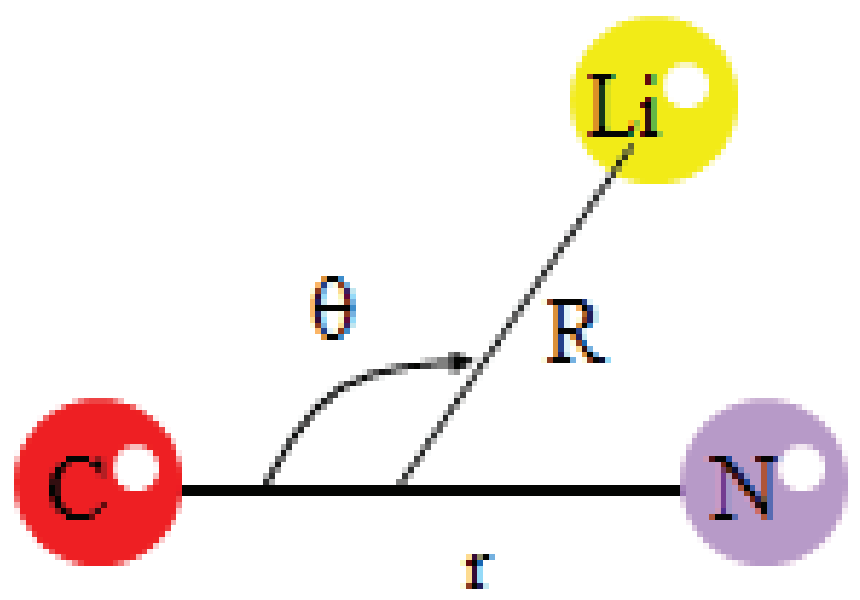

Figura 4.1: Coordenadas de Jacobi para el sistema molecular usadas para describir los movimientos relativos internucleares vibracionales, es decir no se tiene en cuenta el movimiento puramente translacional y se suponen estados no excitados rotacionalmente

$$
H=\frac{P_{R}^{2}}{2 m_{1}}+\frac{P_{r}^{2}}{2 m_{2}}+\frac{P_{\theta}^{2}}{2}\left(\frac{1}{m_{1} R^{2}}+\frac{1}{m_{2} r^{2}}\right)+V_{3 D}(R, r, \theta)
$$

donde $P_{R}, P_{r}$ y $P_{\theta}$ son los momentos conjugados a cada una de las coordenadas.

Por otra parte las masas reducidas $m_{1}$ y $m_{2}$ corrresponden respectivamente al $L i-C N$ y $C-N$ y vienen dadas por:

- $m_{1}=\frac{m_{L i}\left(m_{C}+m_{N}\right)}{m_{L i}+m_{C}+m_{N}}$

- $m_{2}=\frac{m_{C} m_{N}}{m_{C}+m_{N}}$

Usando el hecho ya citado del carácter rígido del enlace $C N$ se puede considerar a la coordenada $r$ desacoplada del resto del sistema y considerar el sistema de $(2+1)$ grados de libertad. Esto nos permite escribir el potencial en la forma

$$
V_{3 D}(R, r, \theta)=V(R, \theta)+M(r)
$$

\subsubsection{Potencial del Sistema 2D}

Para los estudios que tienen como objeto la modelización de algún aspecto de la dinámica de un sistema molecular, es preciso disponer de una superficie de energía potencial lo más ajustada posible a los resultados experimentales. 
En los años 80 se llevó a cabo un importante esfuerzo para llegar a disponer de una expresión analítica de la superficie de energía potencial de este sistema molecular. En 1982, Esser, Tennyson y Wormer [31], presentaron una expresión analítica para el potencial del estado electrónico fundamental del $L i C N$ obtenida por métodos ab initio o de campo autoconsistente que reproduce con una elevada precisión las propiedades espectroscópicas tanto geométricas como energéticas del estado de equilibrio del sistema.

Para el análisis que se emprende en este trabajo, la superficie original de Esser, constituye una buena base de partida para el estudio dinámico del sistema con dos grados de libertad. Necesitamos una expresión de la energía potencial del sistema suficientemente realista y en forma analítica, siendo más relevante su estructura que su precisión en comparación con los resultados experimentales.

Las superficies de energía potencial calculadas por métodos mecanocuánticos ab initio, proporcionan una serie de valores del potencial que pueden ser ajustados a una expresión analítica del potencial lo que facilita en gran medida el cálculo de magnitudes derivadas.

En el caso del sistema $L i C N$ de dos grados de libertad, Esser y colaboradores obtuvieron un mapa de valores de la energía potencial del sistema para distintos valores de $R$ y $\theta$ que ajustaron a un desarrollo de nueve terminos de polinomios de Legendre:

$$
V(R, \theta)=\sum_{\lambda} a_{\lambda}(R) P_{\lambda}(\theta)
$$

donde los coeficientes $a_{\lambda}$ contienen contribuciones de largo y corto alcance del potencial.

En la figura 4.3 se muestra, en forma de curvas de nivel, este potencial $V(R, \theta)$. Como la superficie de energía potencial presenta simetría angular entorno a $\theta=0$ y $\theta=\pi$, se representa, sin pérdida de generalidad, en el intervalo $[0, \pi]$. Observamos que la superficie de energía potencial presenta dos mínimos (pozos ) separados por una barrera. Los dos isómeros estables, que corresponden a los pozos, aparecen en $\theta=0(\operatorname{LiCN})$ y en $\theta=\pi(\operatorname{LiNC})$, siendo este último el más estable. Ambos pozos estan separados por una barrera de energía de $3454 \mathrm{~cm}^{-1}$, siendo su diferencia de energía de 2281 $\mathrm{cm}^{-1}$.

En la tabla 4.1 se muestran parámetros característicos y los puntos más importantes de esta superficie de energía potencial.

Existe un camino de mínima energía [32] que conecta los dos pozos de potencial correspondientes a los dos isómeros (representado mediante una línea discontinua en la figura 4.2). Se ha aproximado este camino de mínima 


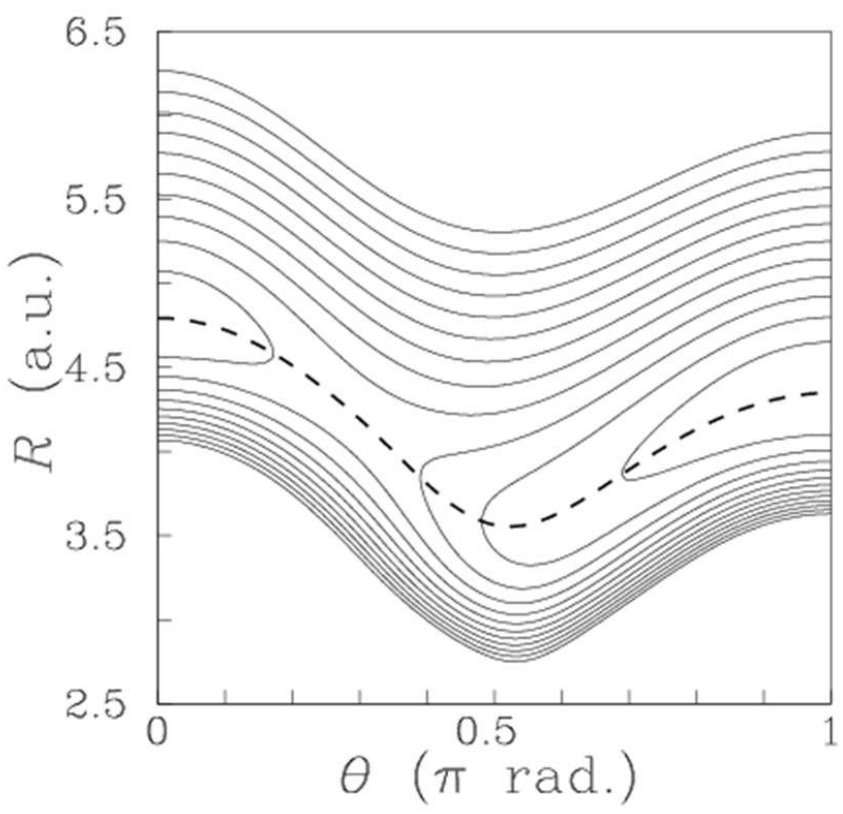

Figura 4.2: Curvas de nivel del potencial $V(R, \theta)$ correspondiente al sistema $\operatorname{LiCN}$ de dos grados de libertad. La linea de trazos representa el camino de mínima energía que conecta los dos pozos

Tabla 4.1: Configuración y energías de los puntos relevantes de la superficie de energía potencial [31] usada en el modelo de dos grados de libertad del sistema molecular LiNC/LiCN

\begin{tabular}{cccl} 
Configuración & $\theta$ (radianes) & $\mathrm{R}(\mathrm{a} . \mathrm{u})$ & $\mathrm{E}\left(\mathrm{cm}^{-1}\right)$ \\
\hline$L i-N C$ & $\pi$ & 4,35 & 0 \\
$L i-C N$ & 0 & 4,795 & 2281 \\
Punto de silla & 0,84491 & 4,30 & 3454 \\
Rellano & 1,91986 & 3,65 & 1207 \\
\hline
\end{tabular}

energía mediante la siguiente expresión (en u.a):

$$
\begin{aligned}
R_{e}(\theta)= & 4,1159+0,25510 \cos \theta+0,49830 \cos 2 \theta \\
& +0,053427 \cos 3 \theta-0,068124 \cos 4 \theta \\
& +0,020578 \cos 5 \theta
\end{aligned}
$$

En la figura 4.3 se muestra el perfil de energía del camino de mínima energía, donde se puede observar de manera clara los puntos relevantes señalados en la tabla 4.1. Como se verá en las siguientes secciones, el camino de mínima energía nos va a permitir obtener representaciones óptimas de la dinámica del sistema. 


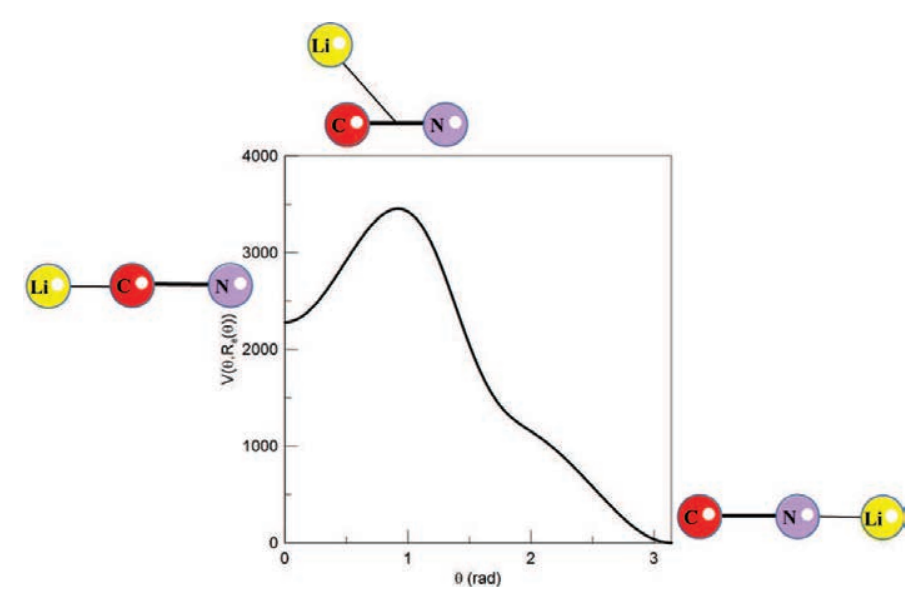

Figura 4.3: Perfil del camino de mínima energía correspondiente al sistema $\operatorname{LiNC} / \operatorname{LiCN}$.

\subsubsection{Potencial del sistema 3D}

Aunque, como se ha indicado, el enlace $C-N$ es muy rígido, es posible que exista movimiento interno en este ión, es decir que la distancia $r$ entre los átomos citados no se mantenga constante, sino que puede oscilar en torno a su posición de equilibrio y que ese movimiento influya en la dinámica del sistema. Se trata de un potencial fuerte que suele ser modelado mediante un potencial de Morse, $M(r)$, que es necesario incluir en la expresión 4.5 para obtener el potencial completo del sistema con 3 grados de libertad. El potencial Morse viene dado por la siguiente expresión:

$$
M(r)=D\left(1-e^{-\alpha\left(r-r_{e}\right)}\right)^{2}
$$

donde la profundidad y anchura del pozo de potencial dependen de los parametros $D$ y $\alpha$ respectivamente.

En el caso del ión $C-N$ estos valores, en u.a., son:

$$
\begin{aligned}
D & =0,29135 \\
\alpha & =1,4988 \\
r_{e} & =2,186
\end{aligned}
$$

En la figura 4.4 se muestra la forma del potencial de Morse del iø'n $C N$ en un entoro del punto de equilibrio $r_{e}=2,186 \mathrm{u}$.a. 


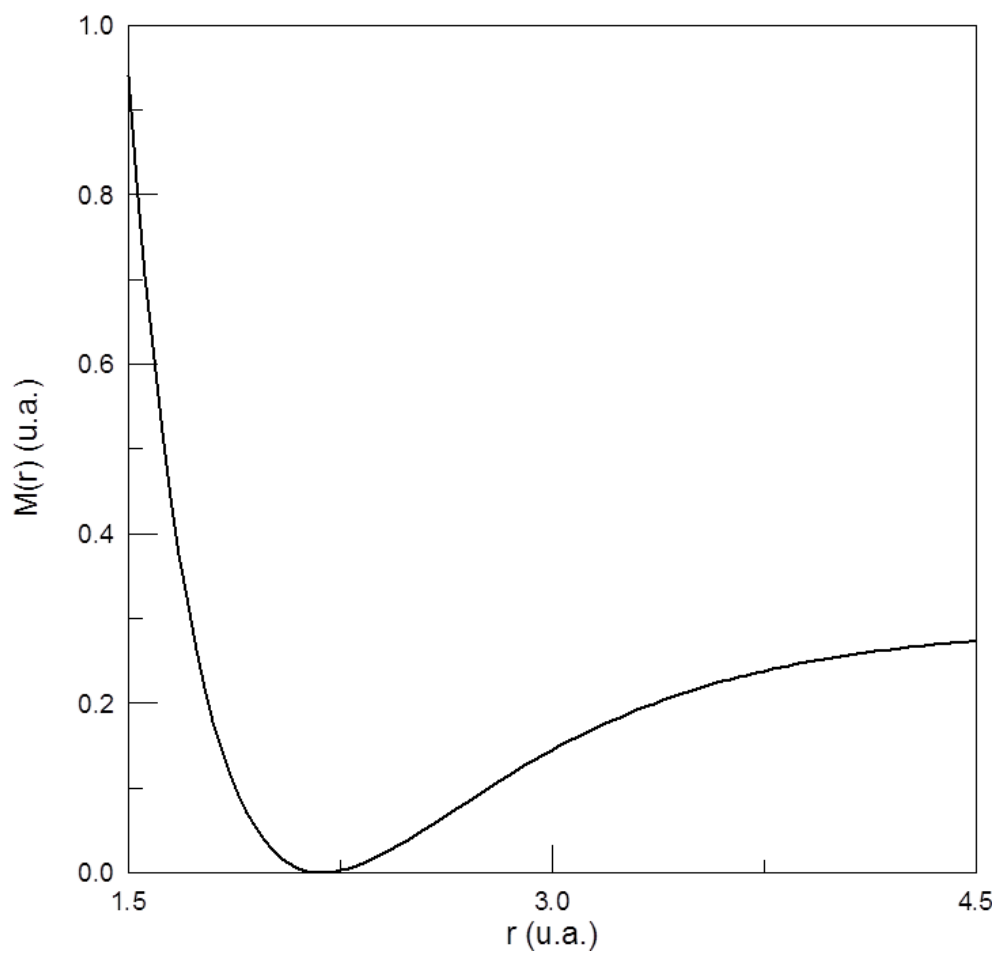

Figura 4.4: Representación del potencial de Morse entre los átomos $\mathrm{C}$ y $\mathrm{N}$ con los valores dados en la expresión 4.9 


\subsection{Ecuaciones de Hamilton y Ecuaciones de Variación}

En esta sección presentamos de forma explícita las ecuaciones del movimiento 2.11 y de variación 2.14 correspondientes al hamiltoniano del sistema molecular en estudo, $L i C N$ de 3 grados de libertad dado en 4.4.

\subsubsection{Ecuaciones de Hamilton del LiNC/LiCN}

Aplicando las ecuaciones del movimiento 2.11 al hamiltoniano 4.4 se obtienen las siguientes ecuaciones de Hamilton, correspondeintes al sistema $\operatorname{LiNC} / \operatorname{LiCN}$ :

$$
\begin{aligned}
& \dot{R}=\frac{P_{R}}{m_{1}}, \\
& \dot{r}=\frac{P_{r}}{m_{2}}, \\
& \dot{\theta}=P_{\theta}\left(\frac{1}{m_{1} R^{2}}+\frac{1}{m_{2} r^{2}}\right), \\
& \dot{P}_{R}=\frac{P_{\theta}^{2} R^{-3}}{m_{1}}-\frac{\partial V(R, \theta)}{\partial \theta}, \\
& \dot{P}_{r}=\frac{P_{\theta}^{2} r^{-3}}{m_{2}}-\frac{\partial M(r)}{\partial r}, \\
& \dot{P}_{\theta}=-\frac{\partial V(R, \theta)}{\partial \theta}
\end{aligned}
$$

Se trata de un sistema de ecuaciones diferenciales autónomo y no lineal. Por otra parte se puede observar que $r$ esta acoplada muy debilmente al sistema ( $r$ y $P_{r}$ solo depende de $P_{\theta}$ y de si mismos). Aprovecharemos este hecho en el capítulo siguiente para obtener una simplificación del análisis del sistema.

\subsubsection{Ecuaciones de Variación}

Aplicando la ecuación de variación 2.14 se obtienen las ecuaciones siguientes ecuaciones, que describen el comportamiento de los vectores de desviación $\left(\Delta R, \Delta r, \Delta \theta, \Delta P_{R}, \Delta P_{r}, \Delta P_{\theta}\right)$ en función del tiempo. 


$$
\begin{aligned}
& \Delta \dot{R}=\frac{\Delta P_{R}}{m_{1}}, \\
& \Delta \dot{r}=\frac{\Delta P_{r}}{m_{2}}, \\
& \Delta \dot{\theta}=P_{\theta} \frac{R^{-3}}{m_{1}} \Delta R+P_{\theta} \frac{r^{-3}}{m_{2}} \Delta r+\left(\frac{1}{m_{1} R^{2}}+\frac{1}{m_{2} r^{2}}\right) \Delta P_{\theta}, \\
& \Delta \dot{P}_{R}=\left(-3 \frac{P_{\theta}^{2} R^{-4}}{m_{1}}-\frac{\partial^{2} V(R, \theta)}{\partial \theta \partial R}\right) \Delta R-\frac{\partial^{2} V(R, \theta)}{\partial \theta^{2}} \Delta \theta+2 \frac{P_{\theta} R^{-3}}{m_{1}} \Delta P_{\theta} \\
& \Delta \dot{P}_{r}=\left(-3 \frac{P_{\theta}^{2} r^{-4}}{m_{2}}-\frac{\partial^{2} M(r)}{\partial r^{2}}\right) \Delta r+2 \frac{P_{\theta} r^{-3}}{m_{2}} \Delta P_{\theta}, \\
& \Delta \dot{P}_{\theta} ;=-\frac{\partial^{2} V(R, \theta)}{\partial \theta^{2}} \Delta \theta-\frac{\partial^{2} V(R, \theta)}{\partial R \partial \theta} \Delta R
\end{aligned}
$$

Se trata de un sistema de ecuaciones lineales, no autónomo (dado que depende del valor de $\vec{z}_{0}(t)$ ). La resolucion de las ecuaciones de variación requiere pues que se resuelva tambien 4.10 . 


\section{Parte II}

\section{RESULTADOS EN 2D}





\section{Capítulo 5}

\section{Resultados del sistema de dos grados de libertad}

En este capítulo vamos a estudiar un modelo 2D del sistema molecular $L i N C / L i C N$ en el que se considera congelada la distancia entre los átomos de $C$ y $N$. Dedicaremos la siguiente sección a la justificación de esta aproximación, dedicando las siguientes a la descripción de las distintas técnicas de análisis utilizadas y la presentación de los resultados obtenidos.

\subsection{Reducción a dos grados de libertad}

A partir de las ecuaciones de Hamilton del sistema completo LiNC/LiCN en 3D, consideramos solo las ecuaciones en las que interviene la coordenada $r$ y su momento conjugado, $P_{r}$ :

$$
\begin{gathered}
\dot{r}=\frac{P_{r}}{m_{2}} \\
\dot{P}_{r}=\frac{P_{\theta}^{2} r^{-3}}{m_{2}}-M^{\prime}(r)
\end{gathered}
$$

Derivando con respecto del tiempo la ecuación 5.1 y sustituyendo $\dot{P}_{r}$ por la ecuación 5.2, se tiene:

$$
\ddot{r}=\frac{P_{\theta}^{2} r^{-3}}{m_{2}^{2}}-\frac{M^{\prime}(r)}{m_{2}}
$$

Que podemos reescribir como:

$$
\ddot{r}+\frac{M^{\prime}(r)}{m_{2}}=\frac{P_{\theta}^{2} r^{-3}}{m_{2}^{2}}
$$


Consideremos la cantidad $\frac{P_{\theta}^{2} r^{-3}}{m_{2}^{2}}$, que denominaremos factor de acoplamiento. Dado el valor relativamente grande de $m_{2}\left(\sim 10^{4} u\right.$.a. $)$ y el valor de $r(r \sim 2,186$ u.a.) podemos asumir que se trata de una cantidad pequeña, del orden de $\sim 10^{-7}$, que se puede despreciar en primera aproximación.

Esto implica que $r$ se comporta en la práctica como un oscilador no lineal al que apenas influyen las variables $R$ ó $\theta$. Es posible entonces promediar el valor de $r$ y considerarlo aproximadamente constante igualándolo a su valor de equilibrio $r=r_{e}=2,186$ u.a. Esto nos permite sustituir el hamiltoniano dado en la eq. (4.4) por el siguiente:

$$
H=\frac{P_{R}^{2}}{2 m_{1}}+\frac{P_{\theta}^{2}}{2}\left(\frac{1}{m_{1} R^{2}}+\frac{1}{m_{2} r_{e}^{2}}\right)+V(R, \theta)
$$

En el que se ha eliminado el termino de $P_{r}$, por ser $r$ constante y por tanto $\dot{r}=\frac{P_{r}}{m_{2}}=0$ y $M\left(r_{e}\right)=0$ como puede obtenerse por simple sustitución.

A partir de este hamiltoniano se obtienen las siguientes ecuaciones del movimiento para el modelo 2D,

$$
\begin{aligned}
& \dot{R}=\frac{P_{R}}{m_{1}} \\
& \dot{\theta}=P_{\theta}\left(\frac{1}{m_{1} R^{2}}+\frac{1}{m_{2} r_{e}^{2}}\right) \\
& \dot{P}_{R}=\frac{P_{\theta}^{2} R^{-3}}{m_{1}}-\frac{\partial V(R, \theta)}{\partial \theta} \\
& \dot{P}_{\theta}=-\frac{\partial V(R, \theta)}{\partial \theta}
\end{aligned}
$$

En este capítulo usaremos esta aproximación, que ha sido utilizada ampliamente [33, 34, 35, 29], reservando el análisis del modelo completo para los capítulos siguientes.

Las trayectorias del sistema simplificado en 2D se obtienen por integración numérica de las ecuaciones del movimiento de Hamilton correspondientes a las ecuaciones (5.6). De esta forma, se obtienen las series temporales $\left(\theta, R, P_{\theta}, P_{R}\right)_{t}$ para cada valor de las condiciones iniciales $\left(\theta, R, P_{\theta}, P_{R}\right)_{0}$ tomadas a la energía, cuya dinámica estamos estudiando. A partir de las series citadas, es decir de las trayectorias en el espacio de fase, generaremos las superficies de sección de Poincaré y los mapas de SALI que se describen en la sección siguiente.

Este análisis permite caracterizar la dinámica vibracional del sistema molecular $\operatorname{LiNC} / \operatorname{LiCN}$ y la reacción química de isomerización $\mathrm{LiNC} \rightleftharpoons \mathrm{LiCN}$ 
que puede tener lugar a energías superiores a la de la barrera de energía potencial, monitorizando el movimiento de la coordenada angular $\theta[36]$.

\subsection{Superficies de sección de Poincaré y Ma- pas de SALIs}

Como elemento de comparación usaremos junto con el indicador SALI, el bien establecido procedimiento de la Superficie de Sección de Poincaré (SSP) especialmente para seleccionar condiciones iniciales en el espacio de fases [33, 34, 35].

\subsubsection{Superficie de sección de Poincaré}

La superficie de sección de Poincaré (SSP) es una herramienta muy útil para visualizar la estructura del espacio de fases de sistemas de dos grados de libertad. En particular, tal y como se describió en la sección 3.1 permite distinguir las zonas caóticas de las regulares.

Para obtener la máxima información de la dinámica del sistema $\mathrm{LiNC} / \mathrm{LiCN}$, en el que puede tener lugar la reacción de isomerización, y por tanto la coordenada angular describa amplios movimientos resulta muy útil en este sistema definir la SSP como la intersección de las trayectorias en el espacio de fases con el camino de mínima energía, $\operatorname{Re}(\theta)$, descrito por la ecuación 4.7. Con esta definición de la SSP, al no ser $\operatorname{Re}(\theta)$ constante y ser una función de la coordenada angular, $\theta$, la correspondiente SSP no conserva el área. Para resolver esta situación, y hacer que la SSP preserve el área, efectuamos la siguiente transformación canónica [34] de las variables:

$$
\begin{aligned}
\rho & =R-R_{e}(\theta), \\
\psi & =\theta, \\
P_{\rho} & =P_{R}, \\
P_{\psi} & =P_{\theta}+\left(\frac{\mathrm{d} R_{e}}{\mathrm{~d} \theta}\right)_{\theta=\psi} P_{R} .
\end{aligned}
$$

De esta forma definimos la SSP como la intersección de la trayectoria con el plano $\rho=0 . P_{\rho}$ se obtiene a partir de la condición de la conservación de la energía dada por Eq. (5.5) tomando el discriminante negativo en la ecuación cuadrática resultante.

Se puede destacar que esta SSP tiene la siguiente simetría:

$$
\begin{aligned}
& \psi \rightarrow 2 \pi-\psi, \\
& P_{\psi} \rightarrow-P_{\psi},
\end{aligned}
$$


por lo que solo necesitaremos representar el intervalo $0 \leq \psi \leq \pi$ en los gráficos correspondientes.

Para visualizar la estructura global del espacio de fases del sisteam utilizaremos las llamadas superficies de sección compuestas. Para ello representaremos en el plano $\left(\psi, P_{\psi}\right)$ las correspondientes SSP de un conjunto de trayectorias elegidas al azar a una energía dada y que cumplan las condiciones indicadas anteriormente de $\rho=0$ y $P_{\rho}$ obtenido en función de la energía.

\subsubsection{Mapas de SALI}

Para analizar de forma global la dinámica del sistema podemos construir distintos mapas que asignen a cada trayectoria $z_{0}$ un valor que las caracterice. Con este objetivo, vamos a escoger el valor correspondiente al mínimo de $S A L I\left(z_{0}, t\right)$ en un intervalo dado de tiempo $T$. Denominaremos $m S A L I_{T}$ a dicho valor.

Formalmente:

$$
m S A L I_{T}\left(z_{0}\right)=\operatorname{mín}\left\{S A L I\left(z_{0}, t\right)\right\}, \quad t \in[0, T]
$$

Donde $z_{0}$ es la condición inicial de la trayectoria y $[0, T]$ el intervalo de tiempo considerado. Dependiendo de como elijamos al conjunto de condiones iniciales podemos definir los distintos mapas.

En general definimos los mapas de SALI como una aplicación $M$ que hace corresponder a cada trayectoria el valor de $m S A L I_{T}$ correspondiente de forma que se cumpla $M: \Omega \rightarrow S$, donde $\Omega$ es el conjunto de valores iniciales que representan a las trayectorias y $S=[0, \sqrt{2}]$ es el conjunto de valores validos de $m S A L I_{T}$.

Mapa de SALI, SM

El Mapa de SALI (SM) es una aplicación que hace corresponder a cada condición inicial de una trayectoria un valor de SALI $s \in[0, \sqrt{2}]$ El conjunto $\Omega_{1}$ de las condiciones iniciales de las trayectorias se escoge sobre la $S S P$ correspondiente a una energía $\mathbf{E}$ determinada. Formalmente el mapa SM se define como la aplicación:

$$
\begin{aligned}
& S M: \Omega_{1} \rightarrow S \\
& \text { donde }: \\
& \Omega_{1}=\left\{z_{0} \mid z_{0}=\left(R, \theta, P_{R}, P_{\theta}\right)\right\} \\
& \theta \in[0,2 \pi] \\
& R=\operatorname{Re}(\theta) \\
& P_{\theta} \in \mathbf{R} \\
& P_{R}=f_{1}(\mathbf{E})
\end{aligned}
$$


Donde $P_{R}$ se calcula para que la trayectoria que pasa por $z_{0}$ cumpla la condición de la conservación de la energía $\mathbf{E}$. El conjunto S está formado por los valores que toma la función $m S A L I_{T}$ definida en 5.9 que corresponde a la trayectoria con la condicion inicial $z_{0} \in \Omega_{1}$.

Mapa Diagrama de Bifurcación con SALI, DBS-SALI

El conjunto de las condiciones iniciales de las trayectorias $\Omega_{2}$ se escoge en función de la coordenada $\mathrm{R}$ y de la energía $\mathbf{E}$.

Formalmente:

$$
\begin{aligned}
& D B S: \Omega_{2} \rightarrow S \\
& \text { donde }: \\
& \Omega_{2}=\left\{z_{0} \mid z_{0}=(R, \mathbf{E})\right\} \\
& R \in \mathbf{R} \\
& P_{R}=0 \\
& \theta=\pi \\
& P_{\theta}=f_{2}(\mathbf{E})
\end{aligned}
$$

\section{Mapa SALI-SSP}

Este mapa consiste en asignar a todos los puntos de una SSP generados por una trayectoria dada por la condición inicial $z_{0} \in \Omega_{1}$ el valor de $m S A L I_{T}$ que corresponde a dicha trayectoria. Denominando $S S P\left(z_{0}\right)$ a la citada superficie tenemos formalmente:

$$
\begin{aligned}
& S A L I-S S P: \Omega_{3} \rightarrow S, \\
& \text { donde }: \\
& \quad \Omega_{3}=\left\{x_{0} \mid x_{0} \in S S P\left(z_{0}\right)\right\} \\
& \left.\quad x_{0}=\left(\psi, \boldsymbol{\Upsilon}_{\psi}\right)\right\},
\end{aligned}
$$




\subsection{Resultados}

\subsubsection{Superficies de sección de Poincaré y SALI}
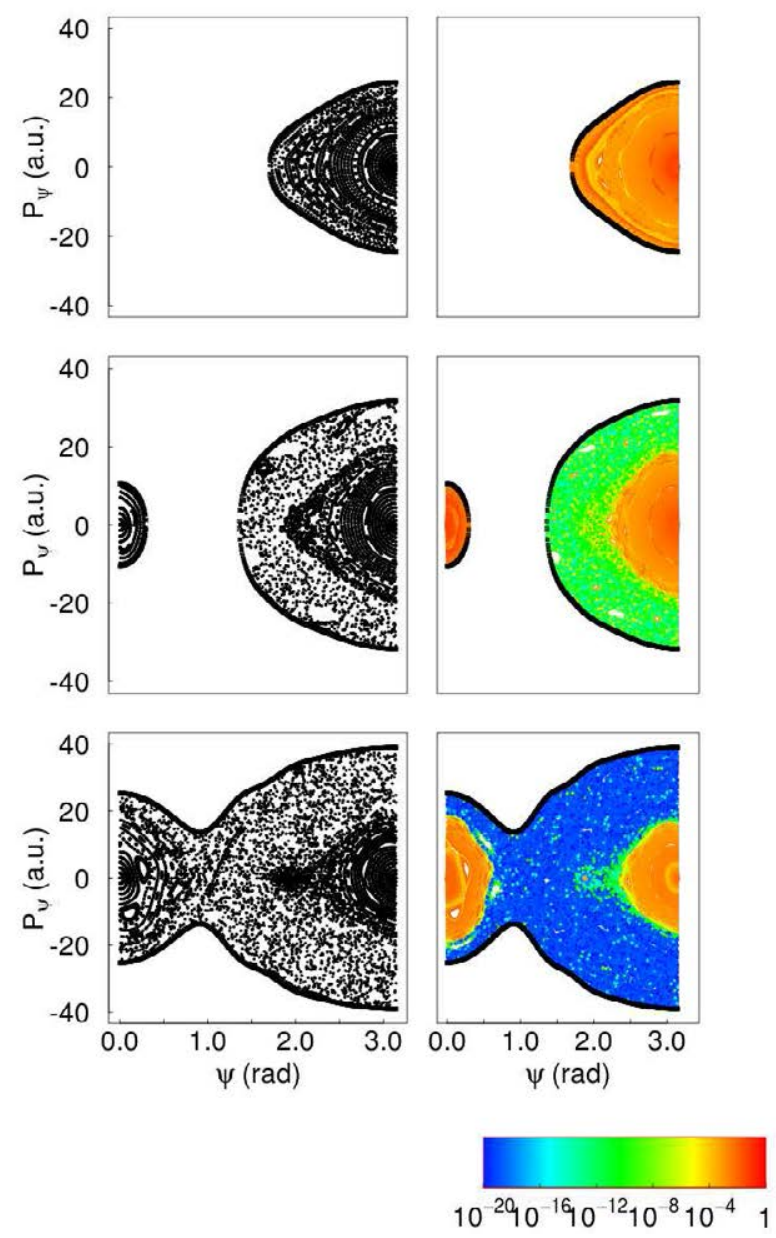

Figura 5.1: Superficies de Sección de Poincaré compuestas (SSP) (izquierda) y los correspondientes mapas SALI-SSP (derecha ) para diferentes valores de la Energía vibracional: $1510,5 \mathrm{~cm}^{-1}$ (arriba), 2549,2 $\mathrm{cm}^{-1}$ (en medio), y $3823,8 \mathrm{~cm}^{-1}$ (abajo). La escala logarítmica de colores, correspondientes a $m S A L I$, se exhibe también en la esquina inferior derecha la figura.

En esta secci'on se presentan los resultados de las Superficies de Sección de Poincaré (SSP) y Mapas de SALI.

En la figura 5.1 mostramos simultáneamente las SSP compuestas y el mapa SALI-SSP del modelo 2D del LiNC/LiCN para tres valores representativos de la energía vibracional del sistema: $E=1510,5 \mathrm{~cm}^{-1}, 2549,2 \mathrm{~cm}^{-1}$, 
y $3823,8 \mathrm{~cm}^{-1}$. En todos los casos, las condiciones iniciales de las trayectorias se escogen al azar sobre la SSP y luego se propagan durante el mismo intervalo de tiempo, $T=2,5 \cdot 10^{5}$ u.a.

En el primer caso, que corresponde a un valor relativamente bajo de la energía $E=1510,5 \mathrm{~cm}^{-1}$, observamos en la SSP que la dinámica es regular, desarrollándose en el entorno del pozo del isómero más estable, Li-NC.

En la fila central, mostramos los resultados para un valor mucho mayor de la energía, $E=2549,2 \mathrm{~cm}^{-1}$. En este caso, el movimiento en el entorno del isómero menos estable $\mathrm{Li}-\mathrm{CN}$, es accesible clásicamente, y además se observa la existencia de grandes regiones del espacio de fase correspondiente al pozo $\mathrm{Li}-\mathrm{NC}$, en las que la dinámica es caótica. Por otra parte, en la región caótica puede observarse una notable acumulación de puntos próximos a la región regular del pozo del Li-NC, que están claramente separados del área caótica restante. La separación entre estas dos partes del mar de caos es un cantoro, cuyo origen y características han sido previamente discutidos en [37].

Finalmente, en el panel inferior, consideramos la energía de $3823,8 \mathrm{~cm}^{-1}$. En este caso la energía es mas alta que la de la barrera de potencial y existen órbitas isomerizantes que conectan los dos pozos del potencial, en las que el átomo de litio rota alrededor del fragmento $\mathrm{C}-\mathrm{N}$. El cantoro continua siendo visible y aparece una isla pequeña de estabilidad en la frontera de la región interior [37].

En la parte derecha de la figura 5.1 se presentan los mapas de SALI-SSP en los que cada punto del mapa se ha representado con un color correspondiente al valor de $m S A L I_{T}$. Los resultados correspondientes a los mapas SALI-SSP muestran esencialmente la misma información que las SSP compuestas.

En las tres energías consideradas, la región coloreada en naranja que corresponde a $\left(m S A L I=10^{0}-10^{-4}\right)$ es la que predomina en los dos pozos, indicando que la dinámica es regular. Las áreas en blanco indican que no se han escogido trayectorias en su interior de forma similar a lo que sucede en las regiones en blanco de la SSP.

Por otra parte, a medida que se consideran valores mayores de la energía, se hacen visibles más áreas oscuras (coloreadas en azul y verde), debido al rápido decrecimiento de $m S A L I\left(\sim 10^{-6}\right.$ y $\sim 10^{-18}$, respectivamente) cuyos valores se asocian al movimiento caótico.

Como puede observarse en SALI-SSP, estos colores naranjas y verde/azules aparecen respectivamente en la misma posición del espacio de fase que los toros KAM o los puntos dispersos en la correspondiente SSP.

No obstante, los mapas de SALI-SSP muestran un espectro de colores más rico, que no solo separa las órbitas regulares de las caóticas [22], sino que también nos permite distinguir entre diferentes grados de caoticidad. De hecho, en los toros de las regiones regulares o cadenas de islas, sus regiones 
estables e inestable aparecen con colores ligeramente diferentes (véase por ejemplo el panel superior de la derecha en las figura 5.1), y en las áreas caóticas la existencia del cantoro se ve marcada por cambios bruscos del valor de SALI, es decir del color en el mapa (de naranja en la región del pozo, a verde en el exterior) en el panel medio a la derecha de la figura 5.1, y del verde claro al azul oscuro en el panel inferior derecha. Una descripción más detallada de este efecto puede observarse en la figura 5.2 que se describirá en detalle en la sección siguiente.

\subsubsection{Evolución temporal del indicador SALI}

En esta sección discutiremos la evolución del inidicador SALI para trayectorias con distintas dinámicas características

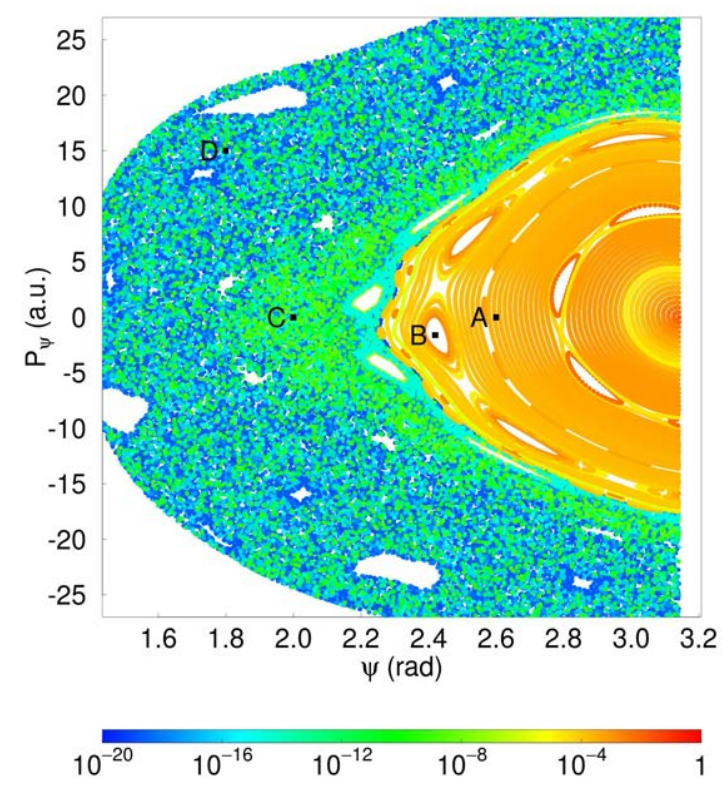

Figura 5.2: Detalle del panel medio de la figura 5.1. Posiciones iniciales (A-D) de las cuatro órbitas discutidas en el texto (Véase la tabla 5.1)

Para analizar con más detalle el último punto de la anterior sección, hemos seleccionado en el mapa SALI-SSP correspondiente a la energía $E=2549,2$ $\mathrm{cm}^{-1}$ cuatro trayectorias $(\mathrm{A}-\mathrm{D})$ con distintas características dinámicas. Las condiciones iniciales en el espacio de las fases de las trayectorias selecionadas se han marcado en la figura 5.2 con las letras A-D. Los valores de las condiciones iniciales y sus características se presentan en la tabla 5.1. 
Tabla 5.1: Condiciones iniciales y características dinámicas de las cuatro trayectorias marcadas (A-D) en la figura 5.2 y mostradas en la figura 5.3.

\begin{tabular}{ccl} 
TRAY. & $z_{0}=\left(\psi_{0}, P_{\psi}^{0}\right)(\mathrm{rad}$, u.a. $)$ & Carácter dinámico \\
\hline $\mathrm{A}$ & $(2.60,0)$ & cuasiperiódica \\
$\mathrm{B}$ & $(2.4232351,-1.6184869)$ & Periódica estable \\
$\mathrm{C}$ & $(2.0,0)$ & caótica en el cantoro \\
$\mathrm{D}$ & $(1.80,15.0)$ & caótica \\
\hline \hline
\end{tabular}
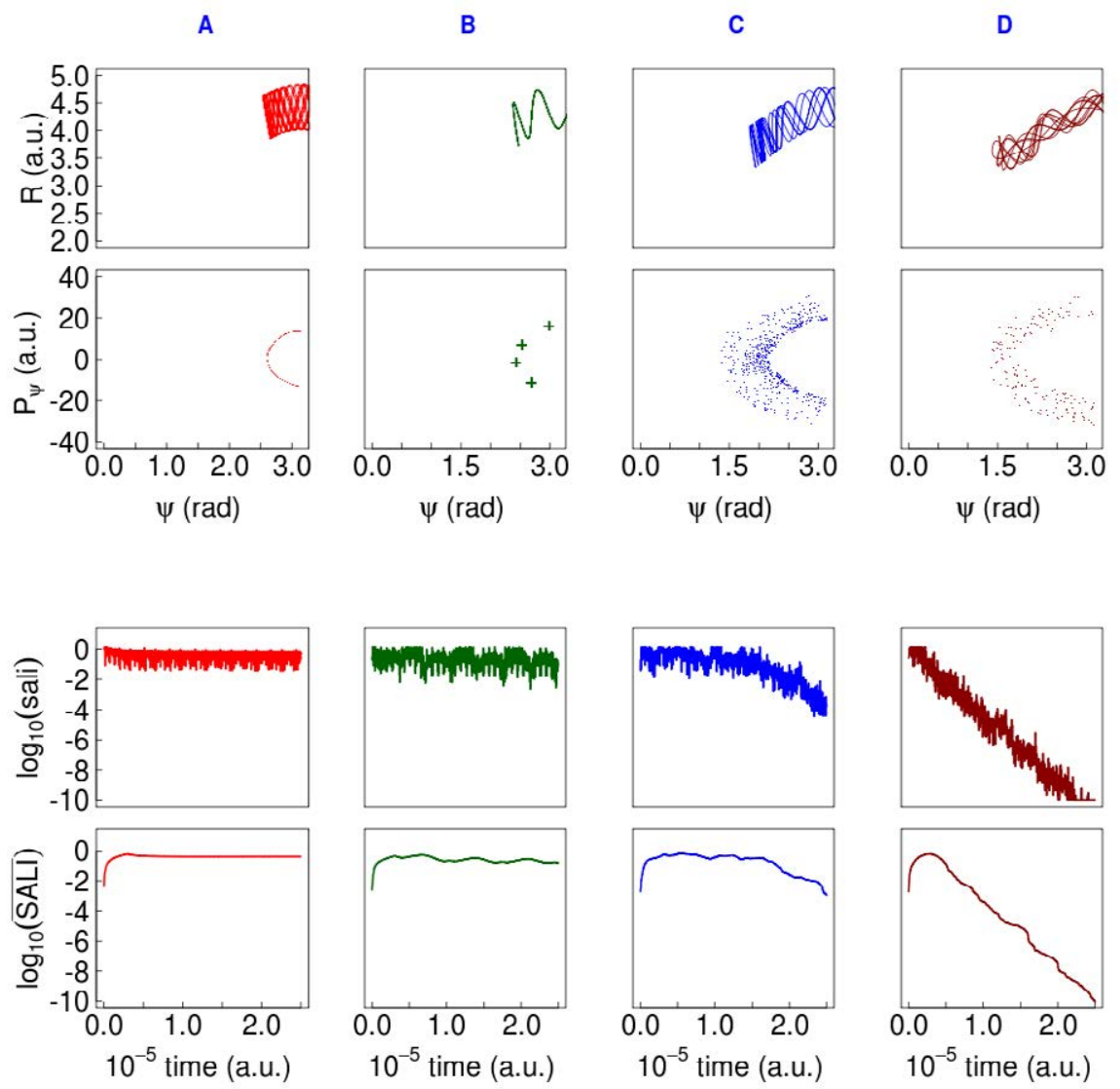

Figura 5.3: Órbita en el espacio de configuración (Fila superior), superficie de sección de Poincaré (Segunda fila ), evolución temporal de $S A L I$ (tercera fila ) y su versión promediada, $\overline{S A L I}$, de las cuatro trayectorias A-D marcadas en la figura 5.2 (Véase también la tabla 5.1 y el texto para los detalles).

Las órbitas correspondientes en el espacio de configuración, SSPs y evolución temporal de $S A L I$ se muestran en las tres filas superiores de la figura 5.3. Dado que esta última magnitud oscila fuertemente, presentamos también en la fila inferior una versión promediada en el tiempo, $(\overline{S A L I})$, sobre una 
ventana temporal, $\tau$, dada por:

$$
\overline{S A L I}\left(z_{0}, t\right)=\frac{1}{\tau} \int_{-\tau / 2}^{\tau / 2} S A L I\left(z_{0}, t+\eta\right) d \eta,
$$

que exhibe más claramente la tendencia general. En nuestros cálculos usamos una ventana con una anchura $\tau=3,5 \cdot 10^{3}$ en u.a.

Un examen de los resultados en la figura 5.3 indica que las trayectorias A y B corresponden a órbitas regulares. Como puede verse en los gráficos de las dos primeras filas, la órbita $A$ es cuasiperiódica, dejando en la SSP una curva cerrada que resulta de la intersección del toro invariante asociado con el camino de mínima energía $\rho=0$. La órbita $B$ por otra parte es una órbita periódica, cuya SSP muestra cuatro puntos fijos (ocho si tenemos en cuenta la simetría de la ecuación (5.8)) que son visitados periódicamente por la órbita. Los valores correspondiente de $S A L I$ y $\overline{S A L I}$ (tercera y cuarta fila en las figuras) son altos para todos los tiempos explorados y aproximadamente constantes indicando claramente su carácter regular.

Por otra parte, las trayectorias C y D corresponden a órbitas de aspecto irregular, que muestran una nube de puntos en la SSP en la zona caótica del espacio de fase. Los valores de $S A L I$ y $\overline{S A L I}$ caen rápidamente a cero, de acuerdo con el caracter caótico de las trayectorias. No obstante, cuando las examinamos con detenimiento, observamos que esas dos trayectorias se comportan de forma diferente.

Así, mientras en el caso C el valor final de $S A L I$ es $\sim 10^{-4}$, en el caso de la órbita $\mathrm{D}$, este valor cae a $\sim 10^{-10}$ en el mismo tiempo. Más específicamente, en la trayectoria $\mathrm{C}$ el valor de $S A L I$ no cambia de forma significativa durante un cierto intervalo de tiempo (del orden de $\sim 10^{5}$ u.a.), mostrando una especie de meseta en la que este valor permanece aproximadamente estacionario.

Esta conducta es equivalente a la acumulación de puntos observada en la SSP, y es debida al hecho de que en este caso hemos escogido una condición inicial (véase la tabla 5.1) muy cercana a la región regular del isómero LiNC. Este tipo de regiones, conocidas como pegajosas ó sticky en la literatura [38], atrapan de forma temporal órbitas cercanas.

Además, esto se refuerza en este caso por el efecto cuello de botella [39] causado por la presencia de un cantoro. Durante este periodo de tiempo, la trayectoria se comporta pues como si fuese regular, y esta es la razón por la que $S A L I$ permanece prácticamente constante.

Se observan también la existencia de mesetas menos prominentes en la evolución temporal de $S A L I$ de la órbita D. Esto es debido a los periodos de tiempo en los que las trayectorias permanecen cercanas a estructuras casi regulares cerca del cantoro siendo afectadas por su influencia dinámica. 


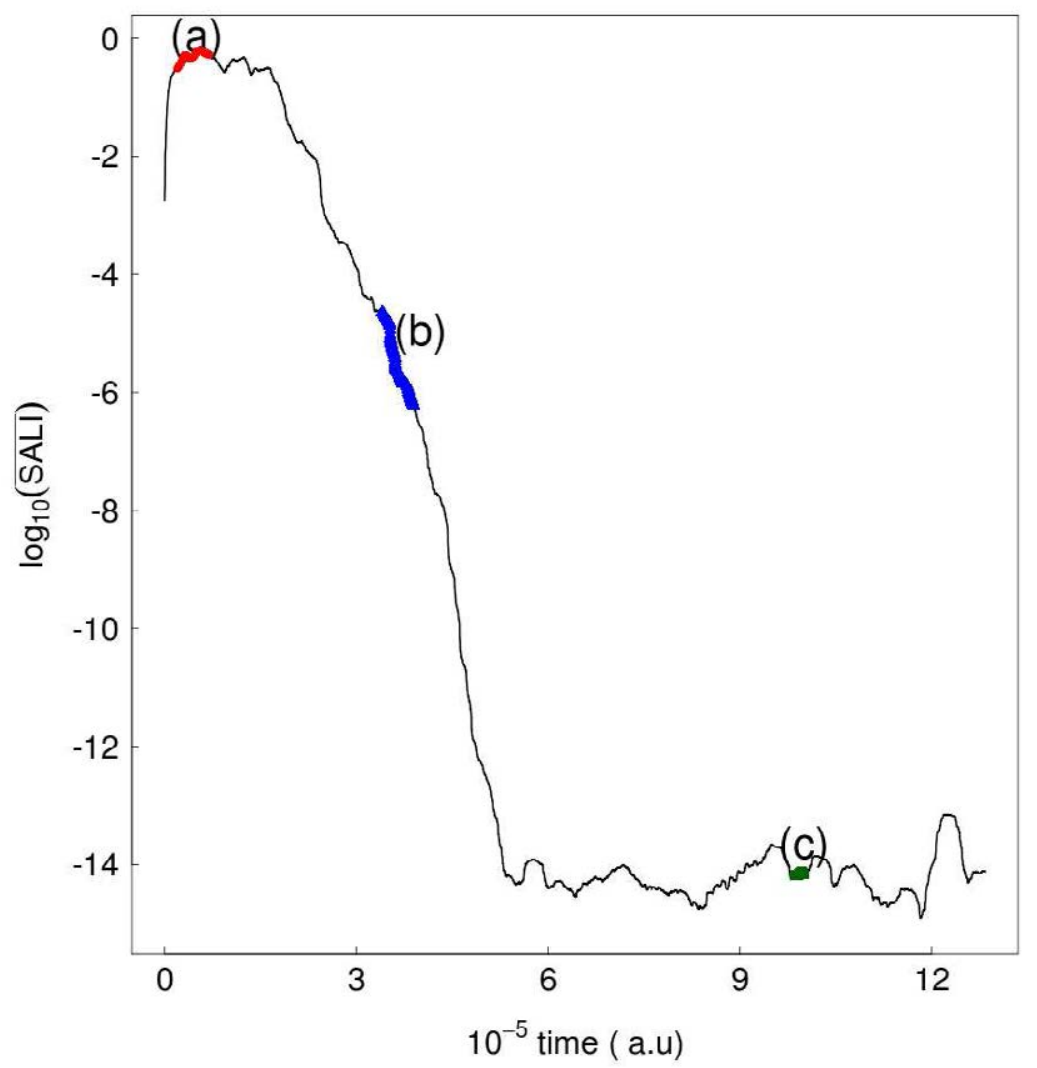

Figura 5.4: Evolución temporal de $S A L I$ de la trayectoria caótica C mostrada en la figura 5.2 y cuyas características se describen en la tabla 5.1 para tiempos largos: $T=1,3 \cdot 10^{6}$ ua.

\section{Comportamiento local de una trayectoria globalmente ergódica en el entorno de un cantoro}

Consideremos ahora con más detalle la información suministrada por SALI observando la trayectoria C, que es globalmente caótica, pero que se inicia en el cantoro y está cerca de la región regular del $L i N C$.

Con este fin hemos propagado la órbita durante mucho más tiempo ( 1,3 . $10^{6}$ u.a.) y los resultados se muestran en la figura 5.4, en la que puede verse que despues de un periodo estacionario, marcado en rojo, el valor de $S A L I$ decrece muy rápidamente (como debería pasar en una trayectoria caótica) hasta que se alcanza $t \sim 5 \cdot 10^{5}$ u.a., momento en el que se estabiliza, aunque presenta algunas obvias oscilaciones.

Para investigar la razón de los diferentes comportamientos de esta curva, hemos seleccionado diferentes porciones de la misma, una al comienzo donde, 

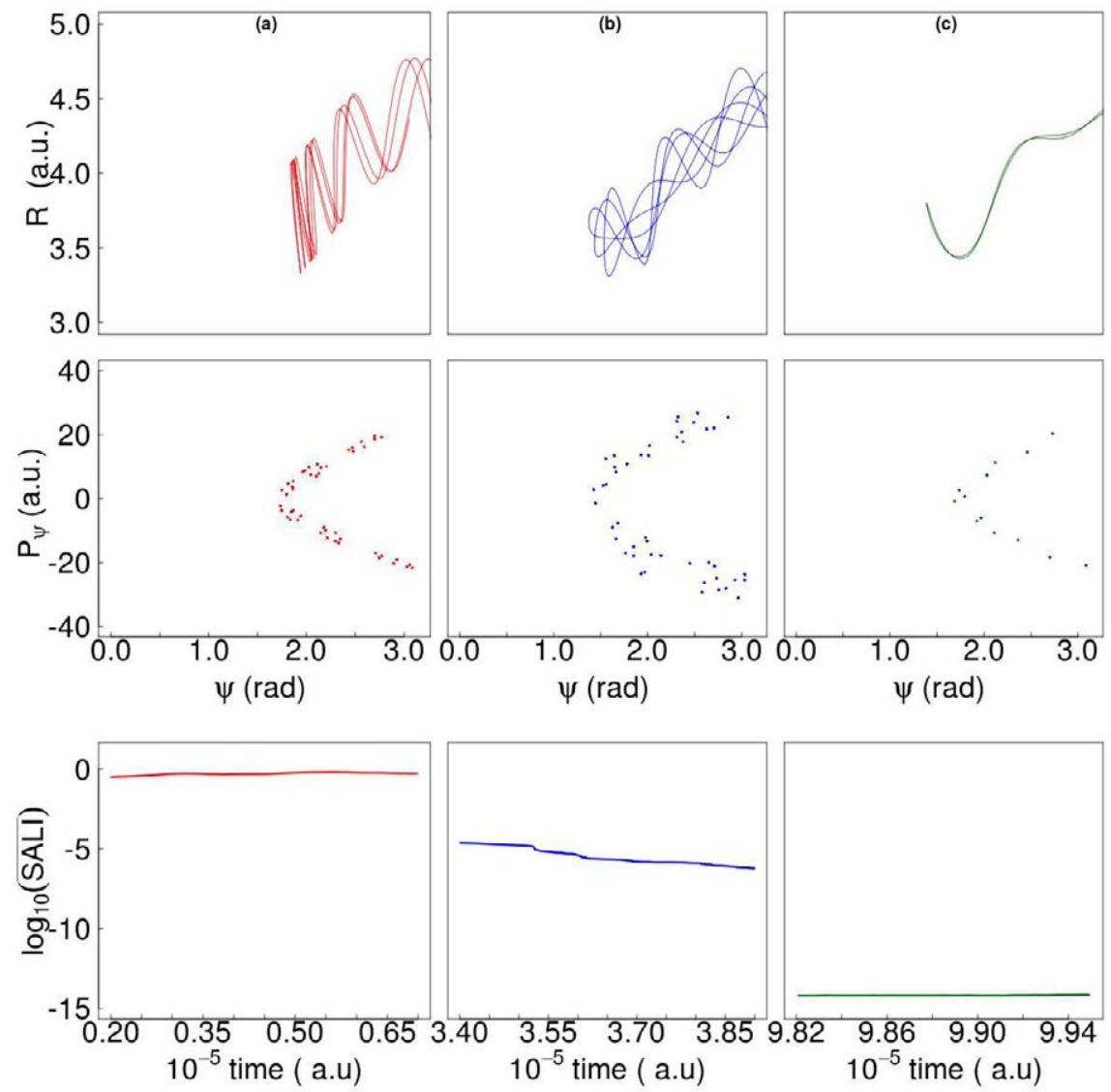

Figura 5.5: Órbita en el espacio de configuración (Arriba), SSP (Fila central), y evolución temporal de $\overline{S A L I}_{T}$ (Abajo) para diferentes porciones de la trayectoria C exhibida en la figura 5.4.

$\overline{S A L I}$ se estabiliza, otra en la que el valor de $\overline{S A L I}$ disminuye rápidamente, y una tercera sobre el área de estabilización final. Se han marcado como (a) rojo, (b) azul y (c) verde, respectivamente.

En las diferentes filas de la figura 5.5 se muestran las porciones correspondientes de la órbita en el espacio de configuración, en el espacio de fase SSP, y la evolución temporal de $\overline{S A L I}$.

Puede observarse que en los intervalos (a) y (c) en los que la trayectoria esta próxima a la región regular del isómero $\operatorname{LiNC}$ el valor de SALI es (aproximadamente) estacionario, mientras que en el (b), correspondiente a la región caótica, cae rápidamente. Esto es, la evolución temporal de $\overline{S A L I}$ nos da una clara indicación de los periodos de tiempo en los que una trayectoria (globalmente) ergódica, está influenciada localmente por estructuras regulares o casi regulares que existen en el espacio de fase mixto de este sistema 


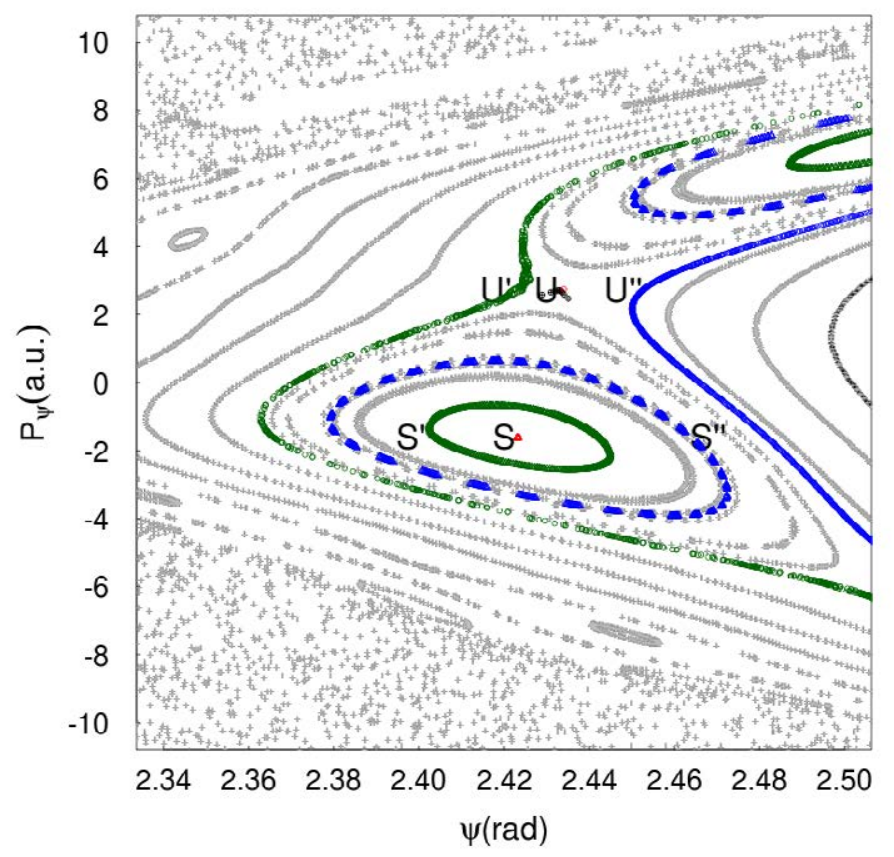

Figura 5.6: Detalle de la superficie de sección de Poincaré compuesta del LiNC/LiCN a $E=2549,2 \mathrm{~cm}^{-1}$ presentado en la figura 5.2 cerca de la trayectoria B.

molecular $[16,18]$.

\section{SALI en el entorno de órbitas periódicas}

Por otro lado investigamos la capacidad de SALI para discernir entre trayectorias con diferentes características dinámicas. Para ello consideramos un par de órbitas periódicas, una estable $(S)$ y otra inestable $(U)$, ligadas en el sentido que han sido originadas por el mismo toro resonante y su entorno, de acuerdo con el teorema de Poincaré Birkhoff (PB). Observaremos así, que SALI también puede ser considerado como un indicador de la estabilidad además de un indicador de caos.

Con este fin, hemos escogido las órbitas periódicas marcadas en rojo ( $\mathrm{S}$ y $\mathrm{U}$ ) en la figura 5.6. Esta es un detalle de la SSP compuesta para LiNC/LiCN a $E=2549,2 \mathrm{~cm}^{-1}$ presentada en la figura 5.2 que corresponde a la región 

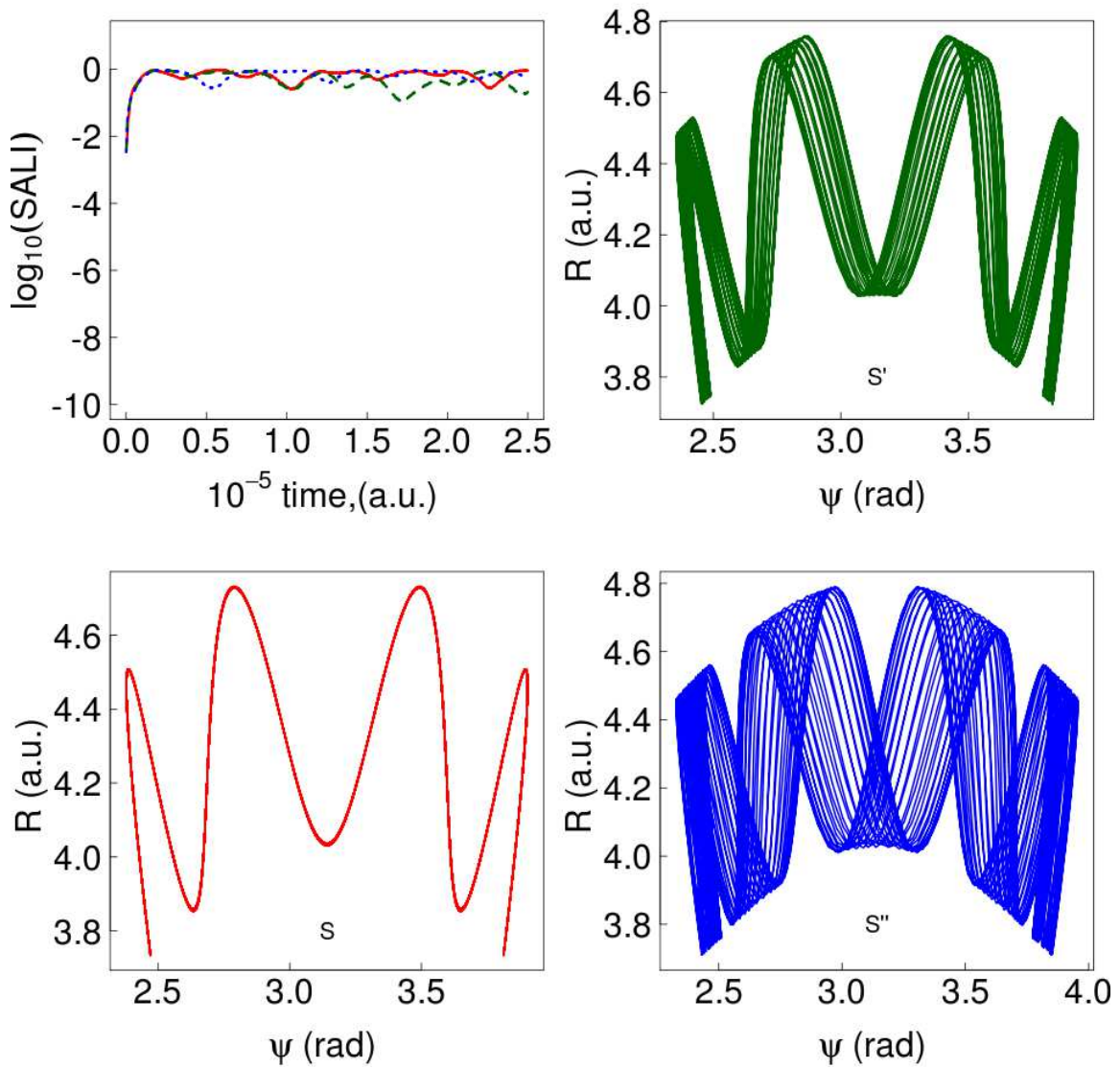

Figura 5.7: Evolución temporal de $\overline{S A L I}$ (arriba izquierda) para las órbitas: estable , $S$ (línea roja continua) y su entorno $S^{\prime}$ (línea verde discontinua) y $S^{\prime \prime}$ (línea azul punteada) marcadas en la figura 5.6. Véase el texto para los detalles. Las órbitas $S, S^{\prime}$ y $S^{\prime \prime}$ se han dibujado en el espacio de configuración.

cerca de la trayectoria B. Hemos marcado en azul y verde, respectivamente, dos trayectorias, $U^{\prime}$ y $U^{\prime \prime}$, cercanas a la órbita inestable y dos órbitas adicionales, $S^{\prime}$ y $S^{\prime \prime}$, marcadas en verde y azul cercanas a la órbita estable haciendo un total de seis órbitas.

Los resultados correspondientes a la elección estable se muestran en la figura 5.7, en la que exhibimos conjuntamente la evolución temporal de $\overline{S A L I}$ y la representación en el espacio de configuraciones de las tres órbitas. Recordamos que estas órbitas son parte de la cadena de islas derivada del teorema PB. Dado que todas estas órbitas están en una región regular del espacio de fase, el valor de $\overline{S A L I}$ oscila alrededor de un valor alto y prácticamente constante (del orden de $\sim 10^{-0,25}=0,56$ ).

El caso de las órbitas en la región alrededor de la órbita periódica inestable 

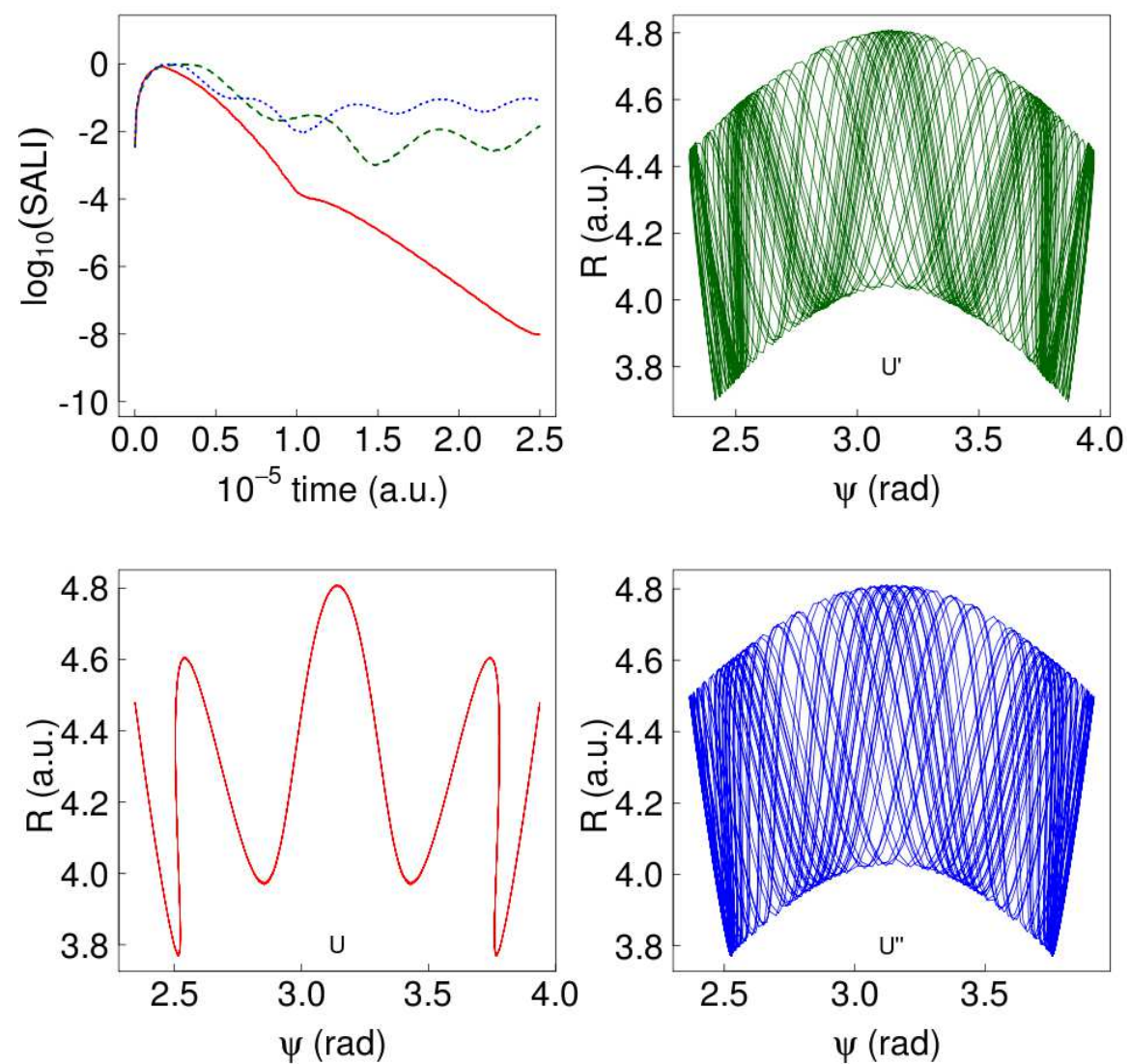

Figura 5.8: Evolución temporal de $\overline{S A L I}$ para las órbitas: inestable $U$, (línea roja continua) y su entorno $U^{\prime}$, (línea verde discontinua) y $U^{\prime \prime}$, (línea azul punteada) marcadas en la figura 5.6. Véase el texto para los detalles. Las órbitas $U, U^{\prime}$ y $U^{\prime \prime}$ se han dibujado en el espacio de configuración.

es un poco más complicado. El espacio de fase cercano a la órbita es caótico, y por tanto se espera que $\overline{S A L I}$ caiga rápidamente. Por otra parte, el caos aquí está restringido a una vía muy estrecha de estocasticidad, estructurada a través de oscilaciones homoclínicas de la órbita central, fuera de la cual la dinámica es regular. El resultado final de esos dos efectos competitivos no puede ser predicho a priori y la respuesta tiene que ser obtenida a partir de cálculos numéricos. Los resultados se muestran en la figura 5.8.

Queremos resaltar que la órbita $U^{\prime}$ (línea verde discontinua) está más cercana a la órbita periódica $U$, y por tanto sigue más o menos la separatriz de esta estructura PB, mientras que la órbita $U^{\prime \prime}$ (línea azul de puntos) está más lejos de $U$ y más cerca de la región regular del toro KAM a la que rodea de estocasticidad. Como resultado, la conducta de $\overline{S A L I}$ es diferente para esas 
órbitas. Mientras que para la órbita periódica inestable presenta un claro decrecimiento exponencial, en las dos trayectorias de su entorno $\overline{S A L I}$ presenta una variación mucho más suave, mostrando pequeñas oscilaciones alrededor de un valor medio, que es análogo al encontrado en la órbita cuasiperiódica A en la figura 5.3.

\subsubsection{Zonas de regularidad y caos en función de la energía}

Una vez estudiada la eficacia de SALI para distinguir entre trayectorias con diferentes características dinámicas, usaremos este indicador para obtener una buena caracterización del espacio de fase del sistema $\operatorname{LiNC} / \operatorname{LiCN}$.

Para ello vamos a calcular dos tipos de mapa basados en este indicador, el mapa DBS-SALI y el mapa SM. Se presenta una visualizacion de los mismos en las figuras 5.9 y 5.10 respectivamente.

\section{Mapa DBS-SALI}

Representamos el mapa DBS-SALI mediante un diagrama en el que se asigna el valor de $m S A L I_{T}$ a cada condición inicial de coordenadas $R$, y $\mathbf{E}$, la energía vibracional del sistema. Cada punto se colorea de acuerdo al valor de $m S A L I_{T}$ de la trayectoria correspondiente a la condición inicial dada por 6.2 , véase la figura 5.9 .

Se puede observar en este diagrama que los valores de $m S A L I_{T}$ son altos (rojo-naranja), y por tanto la dinámica es regular, en las regiones donde la energía es baja o $R$ tiene un valor extremo. Incluso en estas zonas de alta regularidad se observa la existencia de líneas relativamente inestables de color naranja $\left(m S A L I_{T} \leq 10^{-4}\right)$. Se puede comprobar que dichas líneas están correlacionadas con el diagrama usual de bifurcación-continuación que se ha superpuesto (líneas negras) en la figura [40, 41].

A medida que la energía de excitación E se incrementa (2000 - 3200 $\mathrm{cm}^{-1}$ ) aparecen regiones con valores de $m S A L I_{T}$ más bajos (verde o azul claro), en las que sin embargo se encuentran regiones de regularidad (rojo, $m S A L I_{T} \simeq 1$ ) en las que nuevas órbitas periódicas nacen en la bifurcación.

Además, para $E \gtrsim 3200 \mathrm{~cm}^{-1}$ se observa una brusca transición al caos, con predominio de las regiones más oscuras (azul), excepto en los valores bajos y altos de $R$, donde el movimiento continua siendo esencialmente regular (incluso en los valores muy altos de $E$ ). Téngase en cuenta que para esos valores extremos de $R$, la otra coordenada $\psi$ estará próxima al valor de equilibrio cerca del pozo $(\psi \sim \pi)$ debido a la conservación de la energía. 

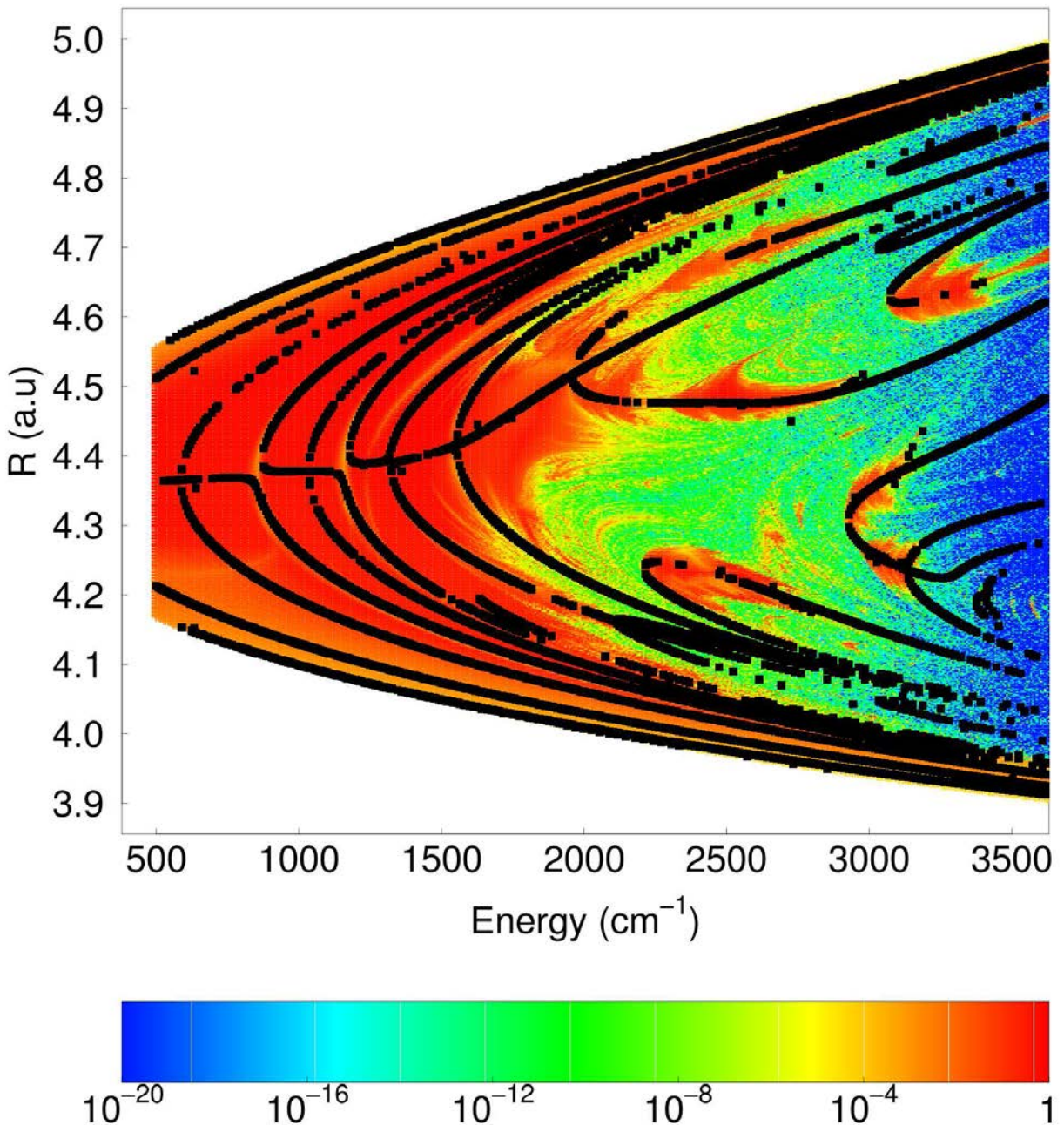

Figura 5.9: Diagrama de Bifurcación-continuación coloreado con SALI para el LiNC (Mapa DBS-SALI) como hemos definido en la sección 5.2.2. Las condiciones iniciales $\Omega_{1}$ dadas en la ecuación (5.10) son $\Delta R=2,2 \cdot 10^{-3}$ u.a. y $\Delta E=7,0 \mathrm{~cm}^{-1}$; Las trayectorias han sido propagadas hasta $T=2,5 \cdot 10^{5}$ u.a. para obtener los valores de $m S A L I_{T}$ en el gráfico usando la escala de colores incluida en la figura. Se muestra también el diagrama usual bifurcación-continuación de órbitas periódicas superpuesto en negro.

\section{Mapa SM}

Consideramos a continuación el mapa $S M$ definido en la sección 5.2.2.

Los resultados para valores diferentes de la energía se muestran en la parte izquierda de la figura 5.10. Para hacer esta figura más cuantitativa, 

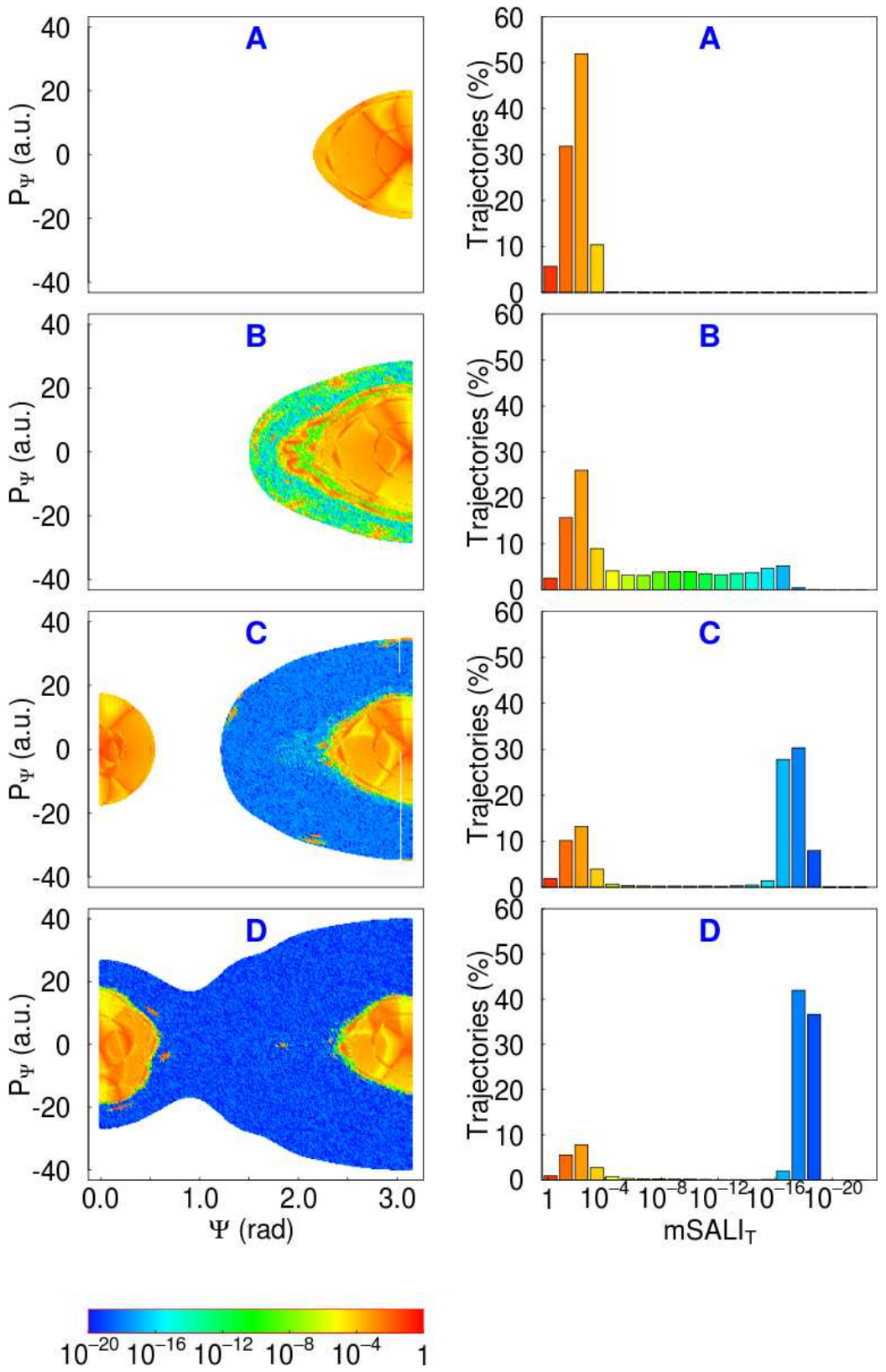

Figura 5.10: Mapas SM (columna izquierda) del sistema $\operatorname{LiNC} / \mathrm{LiCN}$ en 3D a las energías de $E=1000 \mathrm{~cm}^{-1}, 2000 \mathrm{~cm}^{-1}, 3000 \mathrm{~cm}^{-1}$, y $4000 \mathrm{~cm}^{-1}$ mostrados en los paneles $A-B-$ $C-D$ respectivamente. La malla de condiciones iniciales $\Omega_{2}((5.10))$ es $\Delta \psi_{0}=1,570 \cdot 10^{-2}$ rad y $\Delta P_{\psi}^{0}=0,4$ u.a. Las trayectorias han sido propagadas durante $T=2,5 \cdot 10^{5}$ u.a. para obtener los valores de $m S A L I_{T}$ en el gráfico usando la escala de colores que se incluye en la figura. Los histogramas correspondientes de $m S A L I_{T}$ están dibujados en la columna derecha. Téngase en cuenta que el último pico del panel $\mathrm{D}$ incluye todos los valores de SALI menores que $10^{-19}$, y no solo al intervalo $\left(10^{-19}-10^{-20}\right)$ debido a la precisión limitada de los cálculos. 
hemos dibujado en la parte derecha un histograma con la distribución de diferentes valores de $m S A L I_{T}$ (en escala logarítmica), o de forma equivalente sus colores.

Como puede verse, en el valor más bajo de la energía considerado, $E=$ $1000 \mathrm{~cm}^{-1}$ (A, primera fila), todos los puntos están en el rango del amarillo y el naranja, con el valor del indicador $m S A L I_{T}$ centrado en el valor $10^{-3}$ con más del $50 \%$ de las trayectorias localizadas en ese entorno. Esto es una clara indicación de que la dinámica es casi exclusivamente regular a esta energía.

Cuando la energía se incrementa a $E=2000 \mathrm{~cm}^{-1}$ (B, segunda fila) el espacio de fase disponible crece y las nuevas trayectorias que aparecen en esta región presentan valores menores de $m S A L I_{T}<10^{-15}$, asociados a colores en el rango del verde y el azul, que corresponden a trayectorias caóticas. De hecho, observamos la misma distribución que antes para las trayectorias con un valor de $m S A L I_{T}>10^{-6}$ que corresponde a la región regular, mientras que las nuevas trayectorias aparecen más o menos distribuidas igualmente en el resto de los valores de $m S A L I_{T}$.

Además cuando la energía se incrementa a $E=3000 \mathrm{~cm}^{-1}(\mathrm{C}$, tercera fila) las trayectorias irregulares poseen ahora un mayor volumen en el espacio de fase para recorrer y se vuelven más caóticas. Como resultado los colores verdes desaparecen del histograma moviéndose hacia las regiones azules con $m S A L I_{T}<10^{-16}$. Tengamos en cuenta que la distribución de los colores naranja (movimiento regular) permanece en gran medida sin cambios y se centra en valores de $m S A L I_{T} \simeq 10^{-3}$, algo que es notable, especialmente si tenemos en cuenta que se abre una nueva región de estabilidad alredor del pozo del isómero menos estable $\operatorname{LiCN}$ a partir de esta energía.

Este efecto se vuelve incluso más claro para los valores más altos de la energía considerada, $E=4000 \mathrm{~cm}^{-1}$ (D, fila inferior en la figura), donde vemos el incremento de los picos azules en el grupo de las trayectorias más caóticas y al mismo tiempo que el total de las trayectorias regulares han disminuido progresivamente desde las correspondientes a las energías más bajas hasta un mero $10 \%$.

En resumen, se concluye de nuestros cálculos que el espacio de fase que posee el $\mathrm{LiNC} / \mathrm{LiCN}$ consiste en tres regiones diferentes:

- Región de Movimiento Regular: Compuesta por trayectorias que están organizadas en toros KAM alrededor de los pozos de potencial correspondientes a los dos isómeros y por zonas de cadenas de islas.

- Región de Caos Suave: Compuesta por trayectorias irregulares que están influenciadas por la frontera de las regiones regulares mencionadas antes y otras estructuras casi regulares, como los cantoros. 
- Región de Caos Fuerte: Compuesta por trayectorias caóticas que se mueven libremente, recorriendo la parte ergódica del espacio de fase.

Además, la proporción de esas tres regiones cambia con la energía, pasando globalmente de más regular a más caótica, cuando la energía global del sistema aumenta. Este efecto está claramente ilustrado en la figura 5.11, donde se representa el porcentaje de esas regiones en función de la energía de excitación. Estos porcentajes han sido obtenidos a partir de los datos de los histogramas correspondientes a valores de $m S A L I_{T}$ mostrados en la figura 5.10 .

Para contabilizar dichos porcentajes se ha tenido en cuenta los siguientes criterios:

Movimiento Regular (triángulos naranja ) $m S A L I_{T}>10^{-6}$,

Caos suave (cuadrados verdes ) $10^{-6}>m S A L I_{T}>10^{-16}$,

Caos fuerte (círculos azules) $m S A L I_{T}<10^{-16}$.

Puede verse que a energías muy bajas todo el movimiento es regular y la fracción de la dinámica regular (triángulos naranja) se mantiene casi constante hasta una energía de $E=1550 \mathrm{~cm}^{-1}$, mostrando únicamente una disminución muy pequeña en favor de la región de caos suave.

A partir de $E>1550 \mathrm{~cm}^{-1}$ la fracción de dinámica regular cae abruptamente de forma lineal, debido a una importante destrucción de toros (teorema KAM) dando lugar al caos generalizado, hasta $E=2281 \mathrm{~cm}^{-1}$ (marcado con una línea vertical púrpura en la figura) donde la pendiente disminuye debido al hecho de que en este valor de la energía el movimiento sobre la región del segundo isómero $\operatorname{LiCN}$ es clasicamente accesible.

A partir de esa energía, la fracción de dinámica regular permanece constante en un valor aproximado del $30 \%$ en el intervalo $2754-3450 \mathrm{~cm}^{-1}$, (entre las líneas verticales verde y negra), debido a una compensación aparente entre la destrucción de toros regulares en el entorno del isómero $\operatorname{LiNC}$ y la aparición de movimiento regular en el entorno del isómero $\mathrm{LiCN}$.

Finalmente la fracción de movimiento regular continua disminuyendo, esta vez de forma exponencial hasta alcanzar un valor asintótico del $6 \%$. Resaltemos que ese valor no es cero. Esto significa que hay una zona de movimiento regular que no se destruye incluso a muy altos valores de la energía. 


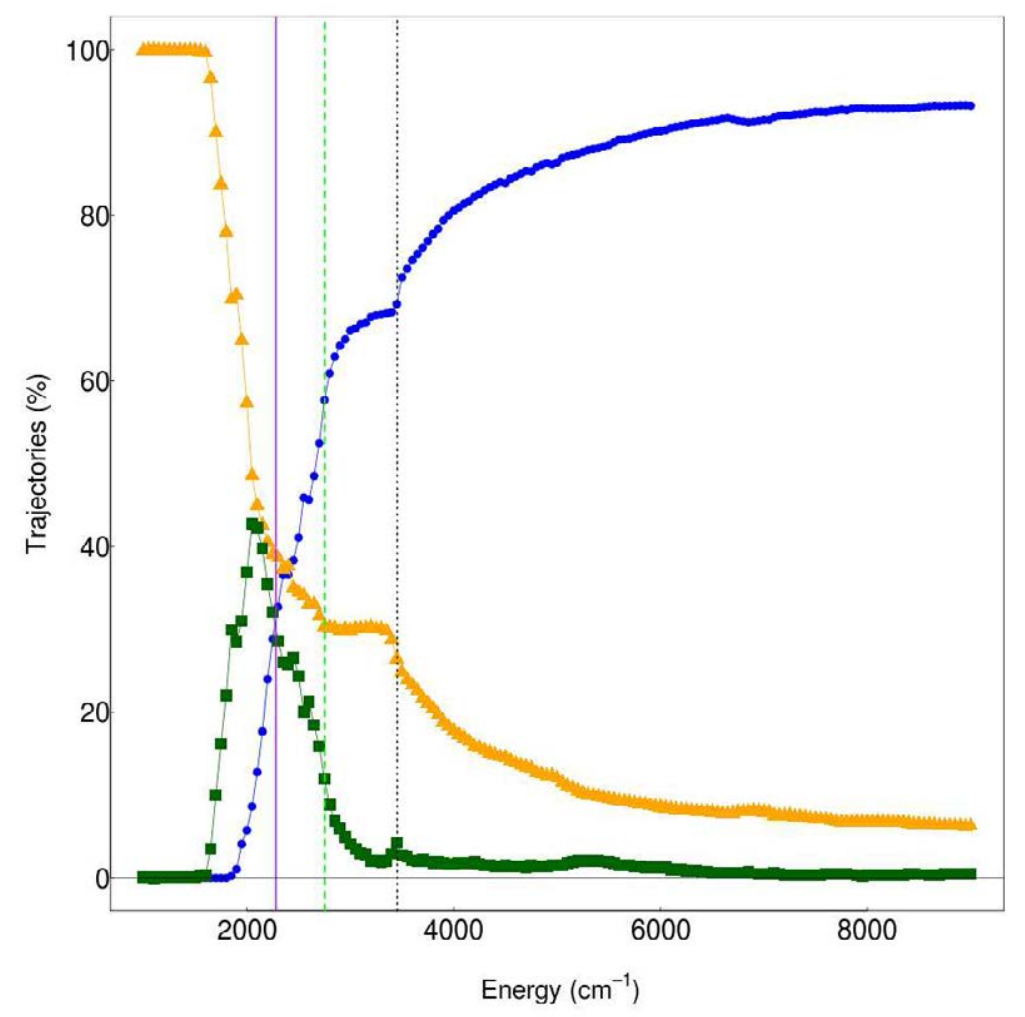

Figura 5.11: Fracción de las tres regiones del espacio de fase del LiNC/LiCN correspondientes a: Movimiento Regular (triángulos naranja) $\left(m S A L I_{T}>10^{-6}\right)$, Caos Suave (cuadrados verdes) $\left(10^{-6}>m S A L I_{T}>10^{-16}\right)$, y Caos Fuerte (círculos azules) $\left(m S A L I_{T}<10^{-16}\right)$. La línea vertical (púrpura) en $2281 \mathrm{~cm}^{-1}$ indica la energía en la que la región del pozo del isómero Li-CN comienza a ser accesible y puede existir movimiento en dicha zona, la línea vertical (verde) en $2754 \mathrm{~cm}^{-1}$ corresponde la energía inicial a la cual hay una compensación entre la destrucción de toros regulares y la aparición de nuevas regiones de regularidad. La línea vertical (negra) en $3450 \mathrm{~cm}^{-1}$ corresponde a la energía de la barrera de energía potencial para la reacción de isomerización.

La conducta de la fracción de caos fuerte (círculos azules) sigue un patrón semejante al anterior, pero obviamente presentando tendencias opuestas. Esto es, hasta $E=1550 \mathrm{~cm}^{-1}$ el porcentaje de movimiento caótico permanece nulo y en ese punto crece súbitamente de forma lineal hasta $E=2281 \mathrm{~cm}^{-1}$, valor de la energía donde la pendiente disminuye un poco, entonces la fracción de caos fuerte permanece constante en el intervalo $E=2450-3450 \mathrm{~cm}^{-1}$ , y finalmente esta magnitud crece hasta alcanzar el valor asintótico del $93 \%$.

Por otra parte, la fracción de caos suave (cuadrados verdes) se comporta 
de forma muy diferente al caos fuerte, presentando características bastante interesantes. Primero, el caos suave solo existe significativamente en el intervalo $E=1600-2900 \mathrm{~cm}^{-1}$. Segundo, el caos suave comienza a crecer significativamente a un valor ligeramente inferior de energía que el caos fuerte, una vez alcanzado un máximo del $21.08 \%$ en $E=1850 \mathrm{~cm}^{-1}$, cae rápidamente a cero manteniendo un valor residual del $\sim 0,6 \%$ entre $E=2900-9000$ $\mathrm{cm}^{-1}$. Esta es la región de la influencia del cantoro discutida en la sección 5.3.1, que atrapa temporalmente trayectorias dentro de él, que luego se mantienen cerca de la frontera de regularidad del isómero LiNC que supone un valor más bajo de $m S A L I_{T}$, y por tanto se detecta caos suave. A medida que la energía aumenta, el cantoro se destruye progresivamente, debilitando su papel de barrera para el flujo de trayectorias a su través. En consecuencia, el efecto que hemos discutido se vuelve progresivamente menos importante, haciendo que la fracción de caos suave tienda a ser despreciable, hasta que solo el caos fuerte sea predominante en la región ergódica del espacio de fase. Esto se muestra en los dos grandes picos del lado derecho del panel D en la figura 5.10. 


\section{Parte III}

\section{RESULTADOS EN 3D}





\section{Capítulo 6}

\section{Sistema con tres grados de libertad}

En este capítulo analizaremos el sistema molecular $\operatorname{LiNC} / \mathrm{LiCN}$ en el caso general de tres grados de libertad. Por tanto el hamiltoniano y las ecuaciones del movimiento que vamos a usar vendrán dados respectivamente por las ecuaciones 4.4 y 4.10 .

Las ecuaciones del movimiento constituyen un sistema no lineal de ecuaciones diferenciales cuya solución está inmersa en un espacio de fase de seis dimensiones. Esto implica, si tenemos en cuenta que se trata de un sistema hamiltoniano conservativo y por tanto la energía total del sistema permanece constante, que las trayectorias se mueven en un espacio de fases de cinco dimensiones. Las SSP no resultan pues una herramienta útil para visualizar la estructura del espacio de fases en el sistema 3D. Sin embargo, como ya hemos visto el indicador SALI es una herramienta útil para caracterizar la naturaleza regular o caótica de las trayectorias de un sistema hamiltoniano independientemene del número de grados de libertad del mismo.

Describiremos una serie de mapas basados en el indicador SALI definidos tanto en el espacio de fases como el de frecuenc y el de energías. Por un lado presentaremos los mapas de SALI equivalentes a los previamente definidos en el caso 2D, en el modelo 3D para estudiar la estructura del espacio de fases del sistema. Por otro lado definimos los mapas de frecuencias y SALI asi como los mapas de energía media combinados con SALI para completar el análisis del sistema 3D.

Dedicaremos la siguiente sección (6.1) de este capítulo a la descripción de los mapas que serán utilizados para el estudio del sistema en tres dimensiones mientras que sección (6.2) se centra en el estudio de evolución de la dinámica del sistema 3D con la energía vibracional total. 


\subsection{Mapas del sistema en 3D}

En esta sección describiremos las herramientas desarrolladas en esta tesis para el estudio del sistema de tres grados de libertad.

Los mapas de SALI $S M_{3 D}$ (6.1.1) y $D B S_{3 D}$ (6.1.2) que se definen a continuación son una generalización directa de los mapas análogos definidos en el capítulo anterior para el modelo de dos dimensiones. Además se presenta los mapas de frecuencias (MF), mapas de frecueencias con SALI (MFS) y mapas de energía media(MEM) en las secciones 6.1.3, 6.1.4 y 6.1 .5 respectivamente.

\subsubsection{Mapa SM en 3D $\left(S M_{3 D}\right)$}

El mapa de SALI en $3 D\left(S M_{3 D}\right)$ es una aplicación que hace corresponder a cada condición inicial $z_{0}$ de una trayectoria el valor de $m S A L I_{T} s \in[0, \sqrt{2}]$. El conjunto $\Omega_{3 D}$ de las condiciones iniciales de las trayectorias se escoge sobre la $S S P$ definida en el caso $2 \mathrm{D}$ correspondiente a una energía $\mathbf{E}$ determinada, para un valor de $P_{r}$ escogido al azar.

Formalmente el mapa $S M_{3 D}$ se define como la aplicación:

$$
\begin{aligned}
& S M_{3 D}: \Omega_{3 D} \rightarrow S \\
& \text { donde }: \\
& \quad S M_{3 D}\left(z_{0}\right)=m S A L I_{T}\left(z_{0}\right) \\
& \Omega_{3 D}=\left\{z_{0} \mid z_{0}=\left(R, r, \theta, P_{R}, P_{r}, P_{\theta}\right)\right\} \\
& \theta \in[0,2 \pi] \\
& R=R e(\theta) \\
& r=2,186 \text { u.a } \\
& P_{\theta} \in \mathbf{R} \\
& P_{r} \in \mathbf{R} \\
& P_{R}=f_{2}\left(\mathbf{E}, z_{0}\right) \\
& S=[0, \sqrt{2}]
\end{aligned}
$$

Donde $f_{2}\left(\mathbf{E}, z_{0}\right)$ asigna a $P_{R}$ el valor necesario para que se cumpla la condición de que la energía total del sistema sea $\mathbf{E}$.

\subsubsection{Mapa DBS en $3 \mathrm{D}\left(D B S_{3 D}\right)$}

El mapa $D B S_{3 D}$ es una aplicación que hace corresponder a cada condición inicial de una trayectoria el valor de $m S A L I_{T} \in[0, \sqrt{2}]$.

El conjunto de las condiciones iniciales de las trayectorias $\Omega_{3 D^{\prime}}$ se escoge en función de la coordenada $\mathrm{R}$ y de la energía $\mathbf{E}$, añadiendo un valor de $P_{r}$ escogido al azar. Formalmente: 


$$
\begin{aligned}
& D B S_{3 D}: \Omega_{3 D^{\prime}} \rightarrow S \\
& \text { donde }: \\
& D B S_{3 D}\left(z_{0}\right)=m S A L I_{T}\left(z_{0}\right) \\
& \Omega_{3 D^{\prime}}=\left\{z_{0} \mid z_{0}=\left(R, r, \theta, P_{R}, P_{r}, P_{\theta}\right)\right\} \\
& R \in \mathbf{R} \\
& r=2,186 \text { u.a. } \\
& \theta=\pi \text { rad } \\
& P_{R}=0 \\
& P_{r} \in \mathbf{R} \\
& P_{\theta}=f_{3}\left(R, \mathbf{E}, z_{0}\right) \\
& S=[0, \sqrt{2}]
\end{aligned}
$$

Donde la función $f_{3}\left(R, \mathbf{E}, z_{0}\right)$ asigna a $P_{\theta}$ un valor para que se cumpla la condición de que la energía total se iguale a $\mathbf{E}$.

\subsubsection{Mapa de Frecuencias (MF)}

Para obtener el mapa de Frecuencias en 3D previamente realizamos un análisis de frecuencias tal y como se ha explicado en la sección 3.3 de esta tesis.

Asignaremos a cada coordenada de la trayectoria definida por la condición inicial $z_{0} \in \Omega_{3 D}$ el valor de la frecuencia correspondiente a la amplitud máxima de cada modo de vibración correspondiente a las funciones:

$$
\begin{aligned}
& f_{R}(t)=R(t)+i P_{R}(t) \\
& f_{r}(t)=r(t)+i P_{r}(t) \\
& f_{\theta}(t)=\theta(t)+i P_{\theta}(t)
\end{aligned}
$$

El resultado es un espacio tridimensional formado por los puntos $\left(\nu_{R}, \nu_{r}, \nu_{\theta}\right)$ correspondientes a los valores de las frecuencias asociadas a cada modo de vibración. Formalmente:

$$
\begin{aligned}
& M F_{3 D}: \Omega_{3 D} \rightarrow \Phi_{3 D} \\
& \text { donde }: \\
& \Phi_{3 D}=\left\{\left(\nu_{R}, \nu_{r}, \nu_{\theta}\right)\right\}
\end{aligned}
$$

Por razones de conveniencia, a veces usaremos la relación entre las frecuencias en lugar de las frecuencias mismas. Esto se traduce en la definición siguiente: 


$$
\begin{aligned}
M F_{2 D} & : \Omega_{3 D} \rightarrow \Phi_{2 D} \\
\text { donde } & : \\
\Phi_{2 D} & =\left\{\left(\nu_{R} / \nu_{\theta}, \nu_{r} / \nu_{\theta}\right)\right\}
\end{aligned}
$$

Introducimos aqui la siguiente notación para trabajar con relaciones de frecuencia: $(l, m, n) \equiv l \nu_{R}+m \nu_{r}+n \nu_{\theta}=0$.

Esta notación sera usada ampliamente.

\subsubsection{Mapa de Frecuencias-SALI (MFS)}

Para completar la información del mapa de frecuencias (MF) definido anteriormente calculamos el valor de $m S A L I_{T}$ para cada condición inicial $z_{0}$ de las trayectorias definidas en el conjunto $\Omega_{3 D}$. Definimos el mapa de Frecuencias-SALI (MFS) asignando el valor de $m S A L I_{T}$ de la orbita que pasa por el valor inicial $z_{0} \in \Omega_{3 D}$ al punto $\left(\nu_{R}, \nu_{r}, \nu_{\theta}\right)_{z_{0}}$ del espacio de frecuencias. Formalmente:

$$
\begin{aligned}
& M F S_{3 D}: \Phi_{3 D} \rightarrow S \\
& \text { donde }: \\
& x_{0}=M F_{3 D}\left(z_{0}\right) \\
& \left.M F S_{3 D}\left(x_{0}\right)=m S A L I_{T}\left(z_{0}\right)\right) \\
& S=[0, \sqrt{2}]
\end{aligned}
$$

De forma análoga definimos el mapa $M F S_{2 D}$.

$$
\begin{aligned}
& M F S_{2 D}: \Phi_{2 D} \rightarrow S \\
& \text { donde }: \\
& x_{0}=M F_{2 D}\left(z_{0}\right) \\
& M F S_{2 D}\left(x_{0}\right)=m S A L I_{T}\left(z_{0}\right) \\
& S=[0, \sqrt{2}]
\end{aligned}
$$

\subsubsection{Mapa de Energía Media $\left(M E M_{3 D}\right)$}

De foma análoga al mapa de frecuencias, se introduce en esta tesis el concepto de Mapa de Energía Media.

Obtenemos el mapa de energía en 3D mediante la aplicación a las orbitas del conjunto $\Omega_{3 D}$ de la sección anterior del calculo de la energía cinetica media correspondientes a sus modos de vibración $E_{R}, E_{r}$ y $E_{\theta}$.

Donde $E_{R}, E_{r}$ y $E_{\theta}$ se definen como: 


$$
\begin{gathered}
E_{R} \equiv \frac{1}{T} \int_{0}^{T} \frac{P_{R}^{2}}{2 m_{1}} d t \\
E_{r} \equiv \frac{1}{T} \int_{0}^{T} \frac{P_{r}^{2}}{2 m_{2}} d t \\
E_{\theta} \equiv \frac{1}{T} \int_{0}^{T} \frac{P_{\theta}^{2}}{2}\left(\frac{1}{m_{1} R^{2}}+\frac{1}{m_{1} r^{2}}\right) d t .
\end{gathered}
$$

(Véase el apendice A)

Para obtener el mapa $M E M_{3 D}$ asignaremos el valor de $m S A L I_{T}$ de la orbita definida por el valor inicial $z_{0} \in \Omega_{3 D}$ al punto $\left(E_{R}, E_{r}, E_{\theta}\right)_{z_{0}}$ del espacio de energías

Formalmente:

$$
\begin{aligned}
& M E M_{3 D}: \Phi \rightarrow S \\
& \text { donde }: \\
& \text { ME } M_{3 D}\left(E_{R}, E_{r}, E_{\theta}\right)_{z 0}=m S A L I_{T}\left(z_{0}\right) \\
& \Phi=\left\{\left(E_{R}\left(z_{0}\right), E_{r}\left(z_{0}\right), E_{\theta}\left(z_{0}\right)\right) \mid z_{0} \in \Omega_{3 D}\right\} \\
& S=[0, \sqrt{2}]
\end{aligned}
$$

\subsubsection{Mapa de Frecuencia Energía $(M F E)$}

Finalmente introducimos un mapa en el que se utilizan simultaneamente los conceptos de frecuencia y la energía media.

Para obtener el mapa $M F E$ asignaremos el valor de $m S A L I_{T}$ de la orbita definida por el valor inicial $z_{0} \in \Omega_{3 D}$ al punto $\left(\nu_{R} / \nu_{\theta}, \nu_{R} / \nu_{\theta}, E_{r}\right)_{z_{0}}$ del conjunto $\Phi_{2 D} \times E_{r}$

Formalmente:

$M F E: \Phi_{2 D} \times E_{r} \rightarrow S$

donde:

$$
\begin{aligned}
& M F E\left(x_{0}, E_{r}\right)_{z 0}=m S A L I_{T}\left(z_{0}\right) \\
& x_{0}=M F_{2 D}\left(z_{0}\right) \\
& S=[0, \sqrt{2}]
\end{aligned}
$$




\subsection{Evolución de la regularidad y el caos con la Energía}

De forma análoga al análisis efectuado con el sistema en 2D en el capítulo anterior, usaremos los mapas definidos en este capítulo para obtener una caracterización del espacio de fase del sistema $\operatorname{LiNC} / \mathrm{LiCN}$ en 3D, centrandonos en esta sección en la relación entre el caracter dinámico del espacio de fases y si evolución con la energía.

\subsubsection{Mapa DBS-SALI}

Representamos el mapa $D B S_{3 D}$ (figura 6.1(a)) mediante un diagrama en el que se asigna el valor de $m S A L I_{T}$ a cada condición inicial de coordenadas $R, P_{r}$ y E. Cada punto se colorea de acuerdo al valor de $m S A L I_{T}$ de la trayectoria correspondiente a la condición inicial $z_{0}$ dada por (6.2). Se observa que se trata de un volumen $3 \mathrm{D}$ en el que las órbitas regulares ocupan las posiciones mas cercanas a la superficie, mientras que en interior existen órbitas caóticas y regulares.

Para dar cuenta de su estructura interna hemos efectuado cortes de la figura con los planos $P_{r}=0$ (b), $R=4,4$ (c) y $E ;=3500$ (d)) que pasamos a analizar a continuación.

En la figura 6.1 (b) (donde efectuamos un corte con el plano $P_{r}=0$ ) se puede observar que los valores de $m S A L I_{T}$ son altos (rojo-naranja), y por tanto la dinámica es regular, en las regiones donde la energía es baja o $R$ tiene un valor extremo. Este comportamiento es muy parecido al que se observa en el modelo de $2 \mathrm{D}$ vease la figura (5.10). Se puede comprobar como a medida que la energía $\mathbf{E}$ se incrementa $\left(2000-3200 \mathrm{~cm}^{-1}\right)$ aparecen regiones con valores de $m S A L I_{T}$ más bajos (verde o azul claro), en las que sin embargo se encuentran regiones de regularidad (rojo, $m S A L I_{T} \simeq 1$ ).

Se observa una brusca transición al caos fuerte cuando $E \gtrsim 3200 \mathrm{~cm}^{-1}$, con predominio de las regiones más oscuras (azul), excepto en los valores bajos y altos de $R$, donde el movimiento continua siendo esencialmente regular incluso a altas de energías.

En la figura 6.1 (c) (correspondiente al corte con el plano $R=4,4$ u.a.) se observa la existencia de zonas regulares casi perfectamente simetricas en torno al eje $P_{r}=0$. Las órbitas son regulares cuando la energía es baja o cuando el valor de $P_{r}$ en valor absoluto resulta lo sufientemente grande.

Por último la figura 6.1 (d) representa el corte tranversal de la figura (6.1) por el plano $E=3500 \mathrm{~cm}^{-1}$. Se trata de una figura practicamente 
(a)

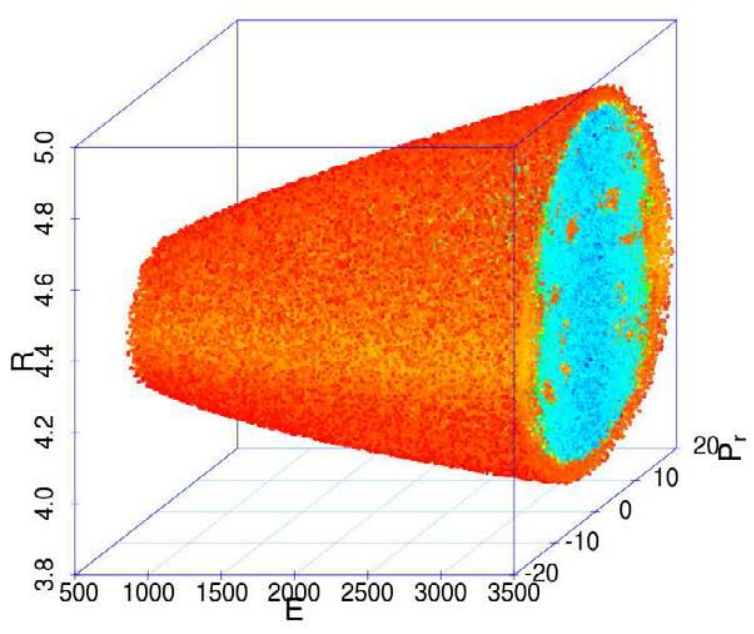

(c)

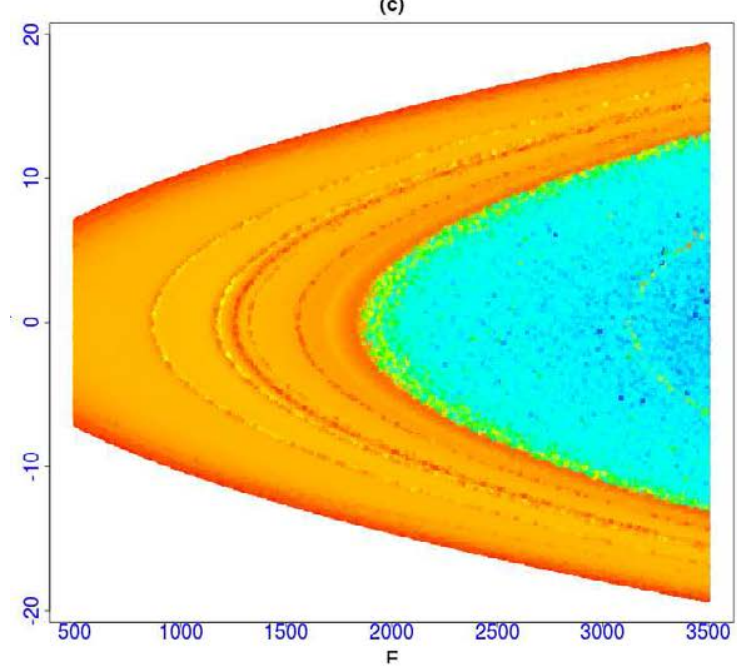

(b)

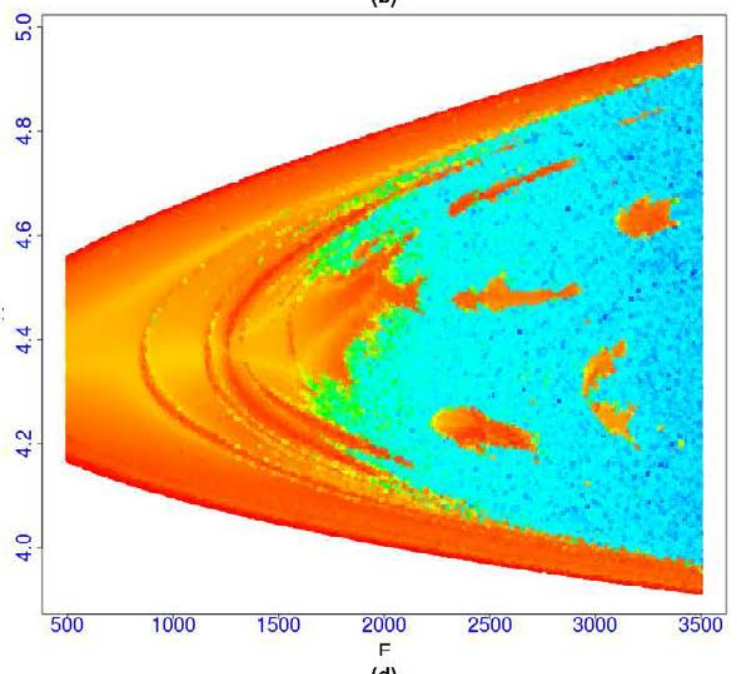

(d)
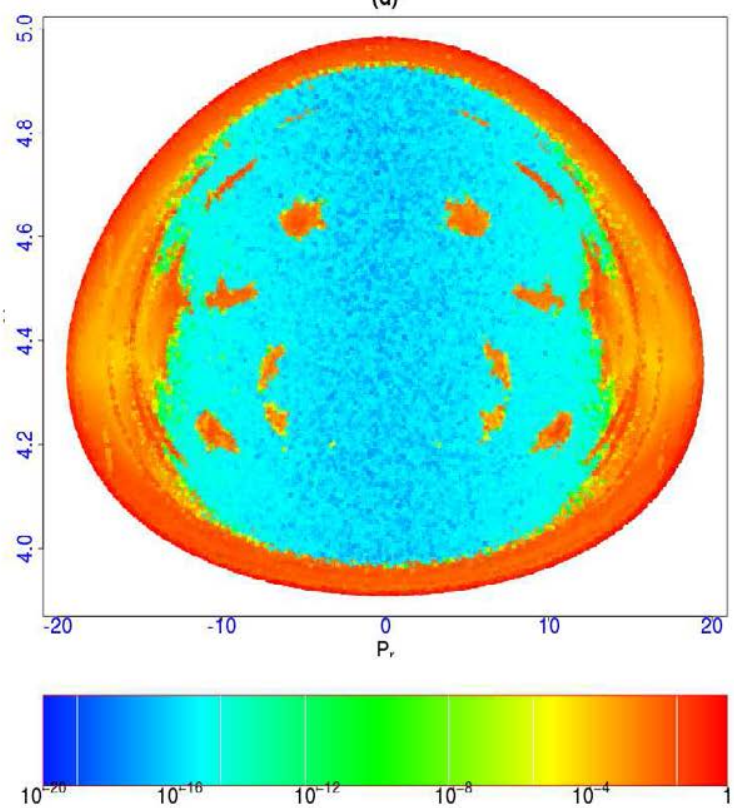

Figura 6.1: Visualizaciones del mapa $D B S_{3 D}$ del $L i N C / L i C N$ en 3D. (a) Mapa 3D; (b) corte con el plano $P_{r}=0$ (c), corte con el plano $R=4,4$ u.a.; (d) corte con el plano $\mathbf{E}=3500 \mathrm{~cm}^{-1}$. Las condiciones iniciales $\Omega_{3 D}$ de acuerdo con la ecuación (6.2) se han obtenido seleccionando de forma aleatoria valores para $R \in[3,5]$ u.a, $P_{r} \in[-40,40]$ u.a. y $E \in[500,3500] \mathrm{cm}^{-1}$. Las trayectorias han sido propagadas durante $T=7,5 \cdot 10^{5}$ u.a. para obtener los valores de $m S A L I_{T}$ que se representan en el gráfico usando la escala de colores incluida en la figura. 
simetrica respecto al eje $P_{r}=0$. Se observa que la mayor parte de las órbitas regulares se sitúan en los limites de la superficie mientras que en el interior se muestran zonas regulares rodeadas por una amplia zona de caos.

Se observa que las zonas regulares se concentran en general en zonas con el valor aboluto de $P_{r}$ grande.

\subsubsection{Mapa SALI-SM $S M_{3 D}$}

Estudiaremos el mapa $S M_{3 D}$ a las energias de 1000, 2000,3000 y $4000 \mathrm{~cm}^{-1}$ que representan un espectro amplio de la energía de las orbitas del sistema.

El mapa se muestra en la columna izquierda de la figura (6.2) mediante un diagrama tridimensional. Las distintas orbitas se representan mediante un punto de coordenadas $\left(P_{r}, P_{\psi}, \psi\right)$ al que se le asigna el valor de $m S A L I_{T}$ mediante el codigo de colores que se muestra en la parte inferior de la figura. En la columna central representamos los planos formados por las órbitas con $P_{\psi}=0$ mientras que en la columna derecha se repesentan los planos correspondientes a orbitas con $P_{r}=0$. Como puede verse en la columna derecha los resultados son muy similares a los obtenidos en el modelo de dos dimensiones (véase figura (5.10)).

En el valor más bajo de la energía considerado, $E=1000 \mathrm{~cm}^{-1}(\mathrm{~A}$, primera fila), todos los puntos están en el rango del amarillo y el naranja correspondiente a valores altos del indicador $m S A L I_{T}$ lo que constituye una clara indicación de que la dinámica es casi exclusivamente regular a esta energía.

El corte con el plano $P_{r}=0$ muestra la existencia de dos zonas de estabilidad que parten del pozo en $\psi=\pi$ asi como la existencia de dos cadenas de islas.El corte correspondiente al plano $P_{\psi}=0$ muestra dos zonas de máxima estabilidad situadas en el entorno del valor absoluto maximo de $P_{r}$ y $\psi=\pi$.

Al incrementar la energía a $E=2000 \mathrm{~cm}^{-1}$ (B, segunda fila) el espacio de fase disponible crece y las nuevas trayectorias que aparecen en esta región presentan valores s de $m S A L I_{T}<10^{-15}$. El corte correspondiente al plano $P_{\psi}=0$ muestra dos zonas disconexas de caos suave y presenta evidencias de la existencia de dos cadenas de islas mientras que el corte con el plano $P_{r}=0$ muestra las dos zonas de caos y una gran cadena de islas.

Cuando la energía se incrementa a $E=3000 \mathrm{~cm}^{-1}$ (C, tercera fila) las trayectorias irregulares poseen ahora un mayor volumen en el espacio de fase 
para recorrer y se vuelven más caóticas. El corte correspondiente al plano $P_{\psi} ;=0$ muestra la aparición de una zona de caos en el entorno de valores bajos del valor absoluto de $P_{\psi}$. El corte con el plano $P_{r}=0$ corresponde a la mayor extension de la zona de caos. A esta energía es posible el movimiento en el entorno del isómero $L I-C N$ siendo este regular.

Este efecto se vuelve incluso más claro para los valores más altos de la energía considerada, $E=4000 \mathrm{~cm}^{-1}$ (D, fila inferior en la figura), donde vemos el incremento del grupo de las trayectorias más caóticas y al mismo tiempo que el total de las trayectorias regulares han disminuido progresivamente. A esta energá ambos pozos están unidos y es posible la presencia de trayectorias isomerizantes en las que el $L i$ puede rotar en torno al fracmento $C N$. El corte correspondiente al plano $P_{\psi}=0$ muestra dos zonas de estabilidad (comn los máximos situados en el entorno de los pozos del potencial) con una zona central casi totalmente caótica. Existe una zona central de regularidad para valores no nulos de $P_{r}$ y $\psi \sim 2,8 \mathrm{rad}$ El corte con el plano $\left.P_{r}=0\right)$ muestra dos zonas de estabilidad situadas en el entorno de los pozos asi como la existencía de cadenas de islas. 

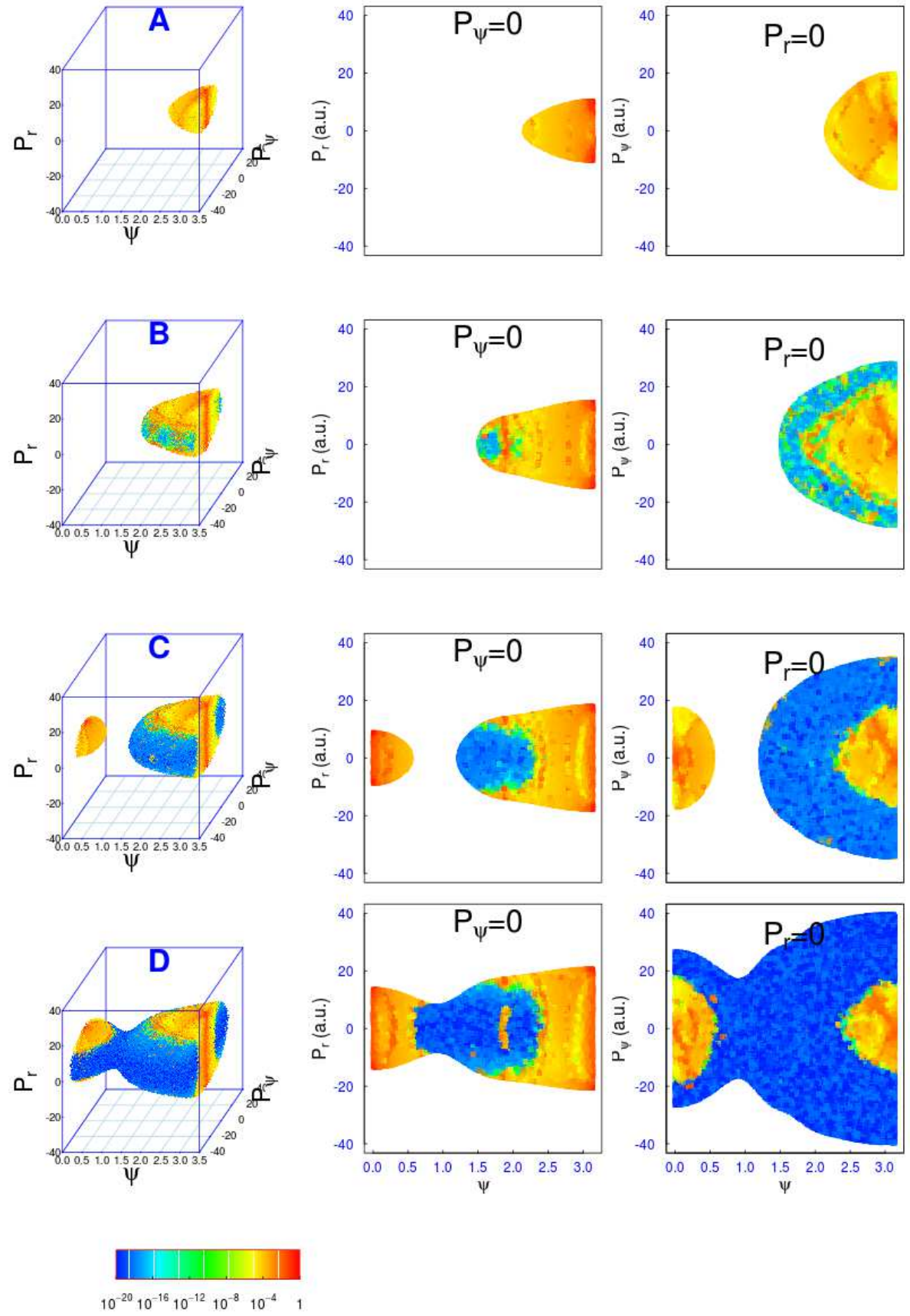

Figura 6.2: Mapas de SALI-SM (columna izquierda) y cortes con los planos $P_{\psi}=0$ (columna central) y $P_{r}=0$ (columna derecha) a las energías de $E=1000 \mathrm{~cm}^{-1}, 2000$ $\mathrm{cm}^{-1}, 3000 \mathrm{~cm}^{-1}$, y $4000 \mathrm{~cm}^{-1}$. La condiciones iniciales $z_{0} \in \Omega_{3 D}$ se han obtenido según la definición 6.1.1. Las trayectorías han sido propagadas durante $T=7,5 \cdot 10^{5}$ u.a. para obtener los valores de $m S A L I_{T}$ en el gráfico usando la escala de colores que se incluye en la figura. 


\subsubsection{Mapas de Frecuencias con SALI $\left(M F S_{3 D}, M F S_{3 D}\right)$}

Para visualizar los mapas de frecuencias con SALI presentamos los diagramas mostrados en la figura 6.3 en la que a cada punto del espacio de frecuencias definido por las coordenadas $\left(\nu_{R}, \nu_{r} \nu_{\theta}\right)$ se le asigna el valor de $m S A L I_{T}$ de cada condición inicial $z_{0} \in \Omega_{3 D}$ representado por el código de colores que se muestra en la parte inferior de la figura.

Hemos analizado el sistema a las energías de $E=1000 \mathrm{~cm}^{-1}, 2000 \mathrm{~cm}^{-1}$, $3000 \mathrm{~cm}^{-1}$, y $4000 \mathrm{~cm}^{-1}$ obteniendo sus mapas corrspondientes cuyos diagramas se muestran en los paneles de la columna derecha de la figura 6.3 (en la columna izquierda se muestran los diagramas correspondientes a los mapas $S M_{3 D}$ como referencia).

En el valor más bajo de la energía $E=1000 \mathrm{~cm}^{-1}$ observamos el carácter regular de todas las órbitas que se agrupan en dos grandes grupo. Por un lado se observa un grupo de órbitas situadas en la resonancia $(1,0,-2)$ mientras que el resto se agrupa en el entorno de la resonancia $(1,-3,0)$

Si incrementamos el valor de la energía a $E=2000 \mathrm{~cm}^{-1}$ observamos la aparición de un grupo de órbitas con caos suave. En el diagrama correspondiente con el mapa $M F_{2 D}$ esto se traduce en el hecho de que la linea del grupo de orbitas principal, entorno de la relacion de resonancia $(1,-3,0))$ se dobla sobre si misma mientras que el grupo de órbitas se ensancha ligeramente. Este ensanchamiento se corresponde con el criterio de caos establecido en [14] y [17]. Por otra parte observamos que las orbitas caóticas se sitúan en las cercanias de dos lineas de resonancia. Esto está tambien de acuerdo con el criterio de caos establecido en [63].

Cuando la energía se incrementa a $E=3000 \mathrm{~cm}^{-1}$ el movimiento en el entorno del pozo correspondiente a $\theta=0$ es accesible. Observamos en el mapa $S M_{3 D}$ que todas las órbitas situadas en ese pozo son regulares $\left(m S A L I_{T}>10^{-4}\right)$. Se comprueba que se sitúan en el diagrama correspondiente al mapa $M F_{2 D}$ en la linea de resonancia $(1,-3,0)$. Por otra parte se comprueba que el número de órbitas caóticas se incrementa.

Finalmente cuando la energía se incrementa a $E=4000 \mathrm{~cm}^{-1}$ los dos pozos son accesibles y estan unidos, por tanto existen orbitas que pueden oscilar en el entorno de os dos isómeros. Existen muchas mas órbitas caóticas que se esparcen en el espacio de frecuencias.

Hay que señalar que, aunque las órbitas caóticas se esparzan en el espacio de frecuencias, no se sitúan de forma aletoria en el mismo. Las órbitas caoticas suelen situarse en el entorno de lineas de resonancia. 

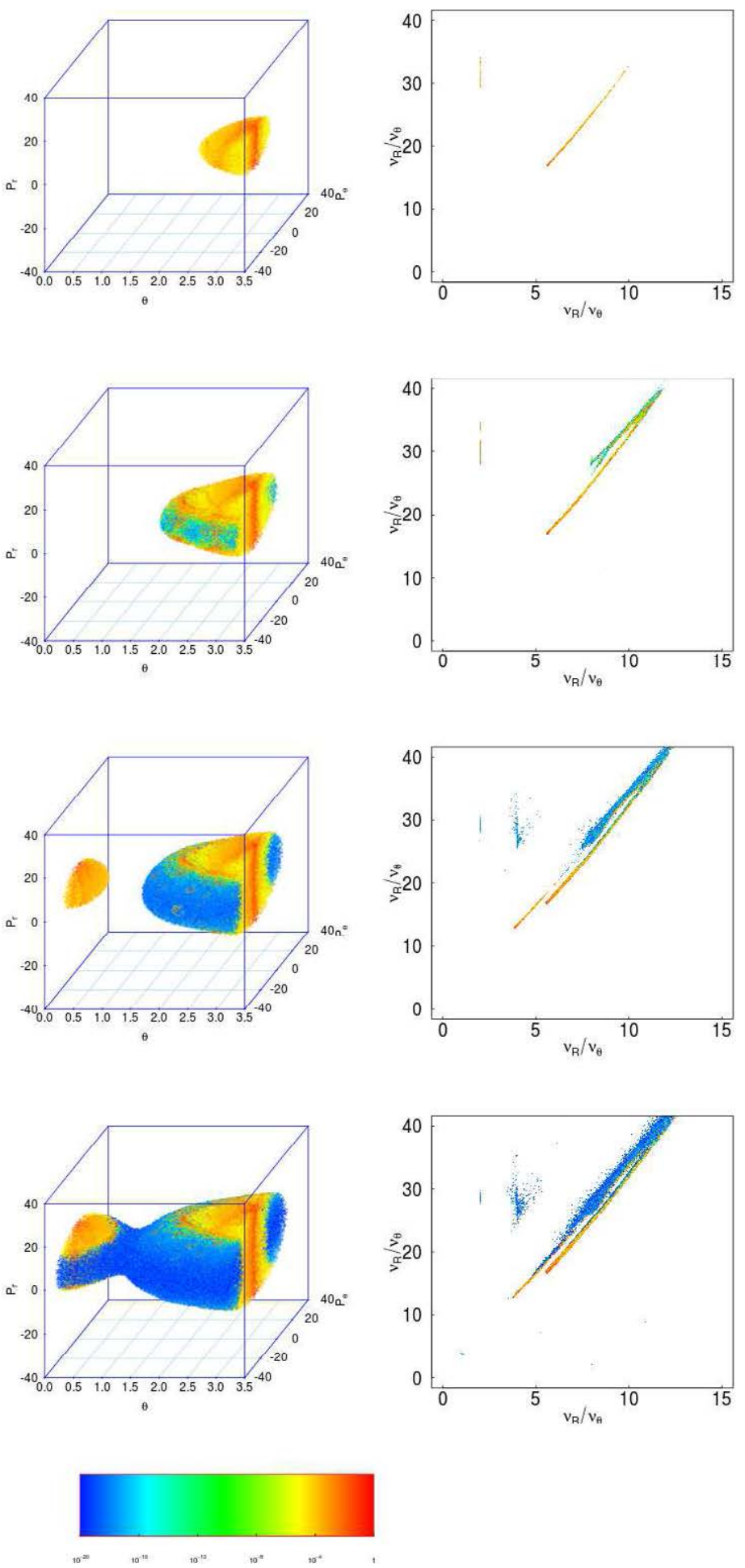

Figura 6.3: Evolución con la energía del mapa $S M_{3 D}$ (izquierda) y del mapa de de frecuencias Mapa $M F S_{2 D}$ (derecha) para el $\operatorname{LiNC} / \operatorname{LiCN} 3 \mathrm{D}$ como hemos definido en la sección 6.1. La condiciones iniciales $z_{0} \in \Omega_{3 D}$ se han obtenido de acuerdo con 6.1.1. Las trayectorias han sido propagadas durante $T=7,5 \cdot 10^{5}$ u.a. para obtener los valores de $m S A L I_{T}$ en el gráfico usando la escala de colores que se incluye en la figura. 


\subsubsection{Mapa de Energía Media $\left(M E M_{3 D}\right)$}

Representamos el mapa $M E M_{3 D}$ mediante un diagrama en el que se asigna el valor de $m S A L I_{T}$ de cada trayectoria correspondiente a la condición inicial $z_{0} \in \Omega_{3 D}$ a las coordenadas $\left(E_{R}, E_{r} \text { y } E_{\theta}\right)_{z_{0}}$ del espacio de energías .

Como en los mapas anteriores hemos analizado las energ1'as de $E=1000$ $\mathrm{cm}^{-1}, 2000 \mathrm{~cm}^{-1}, 3000 \mathrm{~cm}^{-1}$, y $4000 \mathrm{~cm}^{-1}$ que se muestran en los paneles correspondientes de la figura 6.4. A la izquierda de la figura citada se muestran los diagramas correspondientes a los mapas $S M_{3 D}$ como referencia.

Se observa en todas los paneles una clara distincion entre la representación de las órbitas caóticas $\left(S A L I_{T}<10^{-10}\right)$ de color verde/azul de las órbitas regulares $\left(m S A L I_{T}>10^{-10}\right.$ de color rojo/anaranjado. Las primeras se esparcen por el espacio de energías de forma aleatoria mientras que las segundas se integran en planos triangúliformes que incluyen los pozos de potencial.

En el caso correspondiente a $1000 \mathrm{~cm}^{-1}$, las orbitas se organizan en un triángulo plano $A B C$ con los lados $\overline{A C}$ y $\overline{B C}$ aproximadamente rectilineos. Se observa que las orbitas más estables se sitúan en el entorno del vértice $A$ en el que la mayor parte de la energía se concentra en la coordenada $r$. Por otra parte se observa la existencia una familia de órbitas con un valor de $m S A L I_{T}$ mayor que se agrupan en el entorno de la recta $\overline{A B}$. El sistema evita las orbitas cerca del entrono del vértice $C$ que corresponde a órbitas con la mayor parte de la energia en $E_{\theta}$, esto es debido a que el movimiento angular es muy poco rígido. Se observa la existencia de dos familias de órbitas más estables en el entorno de dos líneas $\overline{A^{\prime} B^{\prime}}$ y $\overline{A^{\prime \prime} B^{\prime \prime}}$ paralelas al lado $\overline{A B}$. Dichas órbitas están relacionadas con las resonancias $(1,0,-6))$ y $(1,0,-8)$ entre las variables $R$ y $\theta$.

Cuando incrementamos la energía a $2000 \mathrm{~cm}^{-1}$, observamos que en el triangulo $A B C$ se ha ampliado dada la mayor energía disponible pero que se siguen evitando las orbitas en el vertice $C$. En esa zona se observa la aparición de una zona con caos suave. Como en la energia anterior se puede observar que las órbitas más regulares se sitúan en las inmediaciones del vértice $A$ y en la línea $\overline{A B}$ que corresponde a órbitas con poca energía en $\theta$. Existen tambíen familias de órbitas regulares ligadas a las resonancias $(1,0,-6),(1,0,-8)$ y $(1,0,-10)$ sobre las líneas overline $A^{\prime} B^{\prime}$, overline $A^{\prime \prime} B^{\prime \prime}$ y overline $A^{\prime \prime \prime} B^{\prime \prime \prime}$ respectivamente.

A la energía de $3000 \mathrm{~cm}^{-1}$ ya es accesible energéticamente el pozo correspondiente $\theta=0$ (véase la zona naranja situada a la izquierda de la tercera fila del mapa $S M_{3 D}$ en la figura 6.4, aunque no se da todavia el fenomeno de la isomerización. En el mapa de energía media $M E M_{3 D}$ este hecho se traduce con la existencia de un triángulo adicional $D E F$ formado por órbi- 
tas regulares, semejante pero más pequeño que el triángulo $A B C$ que existe para energías más bajas. El triángulo $A B C$ citado es mucho mayor que en los casos anteriores pero se observa que la zona de caos en el entorno del se ha ampliado considerablemente. Se observa tambien las zonas mas regulares del vértice $A$ y la familia de órbitas regulares ligado a la recta $\overline{A M}$

Cuando la energía llega a $4000 \mathrm{~cm}^{-1}$, ambos pozoa están unidos y posible la presencia de órbitas isomerizantes en las que el átomo de $L i$ puede rotar alrededor del fragmento $C N$. Los triángulos $A B C$ y $D E F$ son mayores que los triángulos análogos a energias más bajas y se observa la aparición de una amplia zona de órbitas con caos fuerte y suave en el entorno de los vértices $C$ y $F$. Como en las energi'as anteriores see observa tambíen las zonas mas regulares del vértice $A$ y las familias de órbitas regulares ligadas a las resonancias $(1,0,-6),(1,0,-8)$ y $(1,0,-10)$ sobre las rectas $\overline{A B}, \overline{A^{\prime} B^{\prime}}$ y $\overline{A^{\prime \prime} B^{\prime \prime}}$ respectivamente. Se percibe tambíen la resonancia $(1,0,-4)$ en la linea $\overline{D^{\prime} E^{\prime}}$ del triángulo $D E F$. 

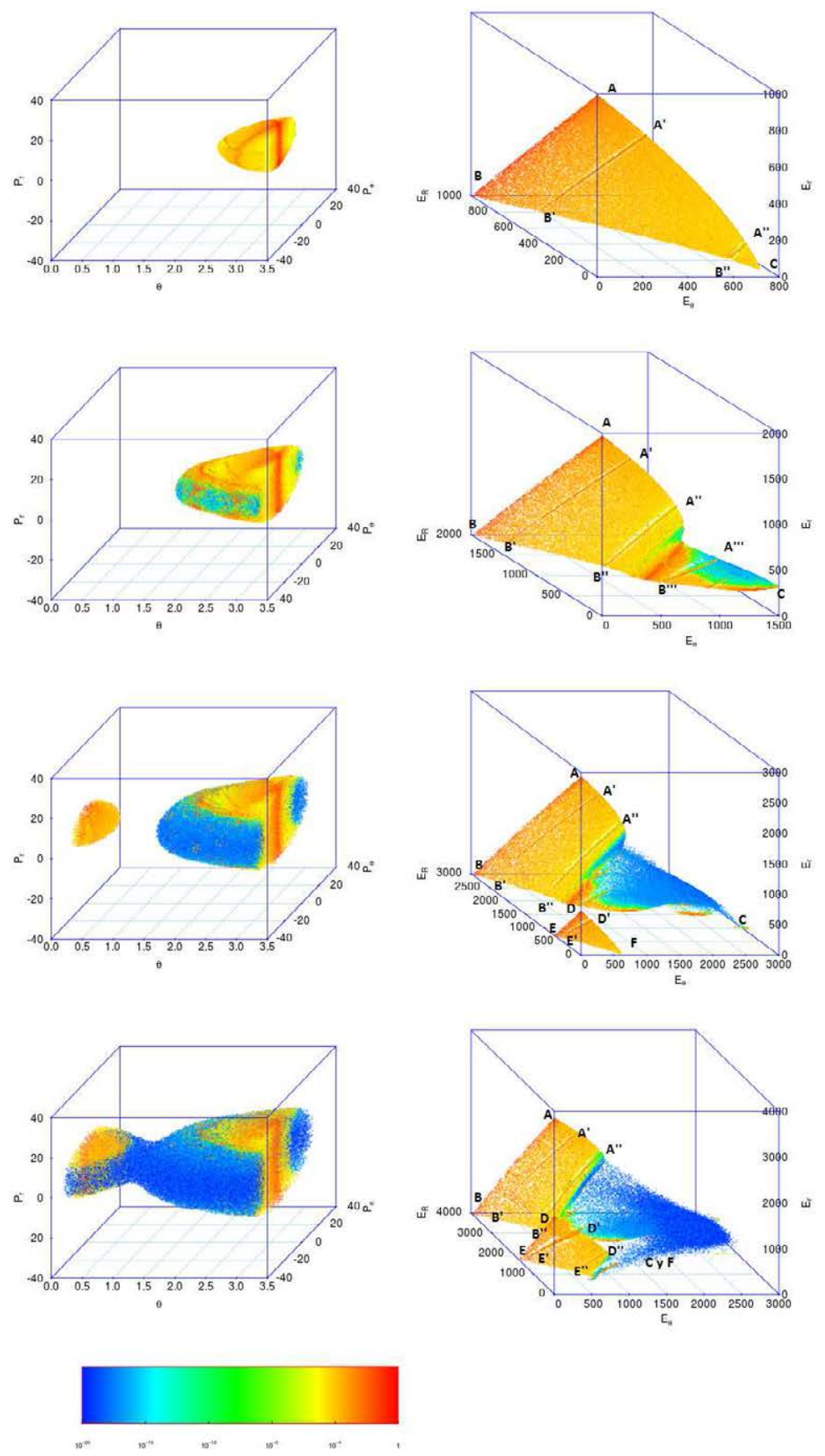

Figura 6.4: Evolución con la energía de los diagramas corrrespondientes a los mapas $S M_{3 D}$ y de energía media, $M E M_{3 D}$, coloreados con SALI para el $L i N C / L i C N$ en 3D como hemos definido en la sección 6.1 . La condiciones iniciales $z_{0} \in \Omega_{3 D}$ se han obtenido seleccionando de forma aletoria valores para $\psi, P_{\psi}$ y $P_{r}$ obteniendose el resto de los valores de acuerdo con 6.1.1. Las trayectorías han sido propagadas durante $T=7,5 \cdot 10^{5}$ u.a. para obtener los valores de $m S A L I_{T}$ en el gráfico usando la escala de colores que se incluye en la figura. 


\subsubsection{Mapa de Frecuencia Energía $(M F E)$}

Representamos el mapa $M F E$ mediante un diagrama en el que se asigna el valor de $m S A L I_{T}$ de cada trayectoria correspondiente a la condición inicial $z_{0} \in \Omega_{3 D}$ a las coordenadas $\left(\nu_{R} / \nu_{t}\right.$ heta $\left., \nu_{r} / \nu_{\theta}, E_{r}\right) z_{0}$.

Como en los mapas anteriores hemos analizado las energ1'as de $E=1000$ $\mathrm{cm}^{-1}, 2000 \mathrm{~cm}^{-1}, 3000 \mathrm{~cm}^{-1}$, y $4000 \mathrm{~cm}^{-1}$ que se muestran en los paneles correspondientes de la figura 6.5. A la izquierda de la figura se muestran los diagramas correspondientes a los mapas $S M_{3 D}$ como referencia.

Como en los casos anteriores se observa en todas los paneles una clara distincion entre la representación de las órbitas caóticas $\left(S A L I_{T}<10^{-10}\right)$ de color verde/azul de las órbitas regulares $\left(m S A L I_{T}>10^{-10}\right.$ de color rojo/anaranjado. Las primeras se esparcen por el espacio de forma aleatoria mientras que las segundas se integran en figuras planas.

En el caso correspondiente a $1000 \mathrm{~cm}^{-1}$, las orbitas se organizan en un triángulo plano $A B C$ con los lados $\overline{A B}$ y $\overline{B C}$ aproximadamente rectilineos y en una familia lineal unidimensioal de órbitas $\overline{D E}$ cuya posición es paralela al lado $\overline{A C}$. Las órbitas situadas en el triángulo $A B C$, ampliamente mayoritarias, estan con la relación de resonancia $(2,-6,-1)$ mientras que la familia de las orbitas en $\overline{D E}$ tiene la relación de resonancia $(1,0,-2)$. La orbitas mas estables se sitúan en el entorno de la linea $\overline{A B}$.

Cuando incrementamos la energía a $2000 \mathrm{~cm}^{-1}$, observamos que el número de las órbitas situadas en el triángulo $A B C$ se ha ampliado dada la mayor energía disponible pero que la linea $\overline{A C}$ se ha quebrado en dos $\overline{A C^{\prime}}$ y $\overline{C^{\prime} C}$ mientras que la antigua línea $\overline{D E}$ se retuerce sobre si misma y acaba en una pequeña zona de caos. Aparece tambien caos en el entrono de la linea $B C$. Las órbitas con mayor estabilida se sitúan en las líneas $\overline{A B}$ y $\overline{C^{\prime} C}$.

Cuando llegamos a la energía de $3000 \mathrm{~cm}^{-1}$, como ya hemos indicado es accesible energéticamente el pozo correspondiente $\theta=0$ (véase la zona naranja situada a la izquierda de la tercera fila del mapa $S M_{3 D}$ en la figura 6.5, aunque no se da todavia el fenomeno de la isomerización . En el mapa de frecuencia energía $M F E$ este hecho se traduce en la existencia de un triángulo adicional $F H G$ formado por órbitas regulares,muy semejante pero más pequeño que el triángulo $A B C$ que existe para energías más bajas y de otra línea $\overline{I J}$ semejante a la linea $\overline{D E}$ de los otros niveles de energía. Se observa que la zona de caos en el entorno del lado $\overline{B C}$ se ha ampliado considerablemente, mientras que en el entrono de la linea $\overline{D E}$ aparecen dos zonas de caos adicional.

Cuando la energía llega a $4000 \mathrm{~cm}^{-1}$, ambos pozos están unidos y posible la presencia de órbitas isomerizantes en las que el átomo de $L i$ puede rotar alrededor del fragmento $C N$. Las figuras $A B C$ y $D E F$ son mayores que los 
triángulos análogos a energias más bajas y se observa la aparición de una amplia zona de órbitas con caos fuerte y suave en el entorno de los vértices $C$ y $F$. 

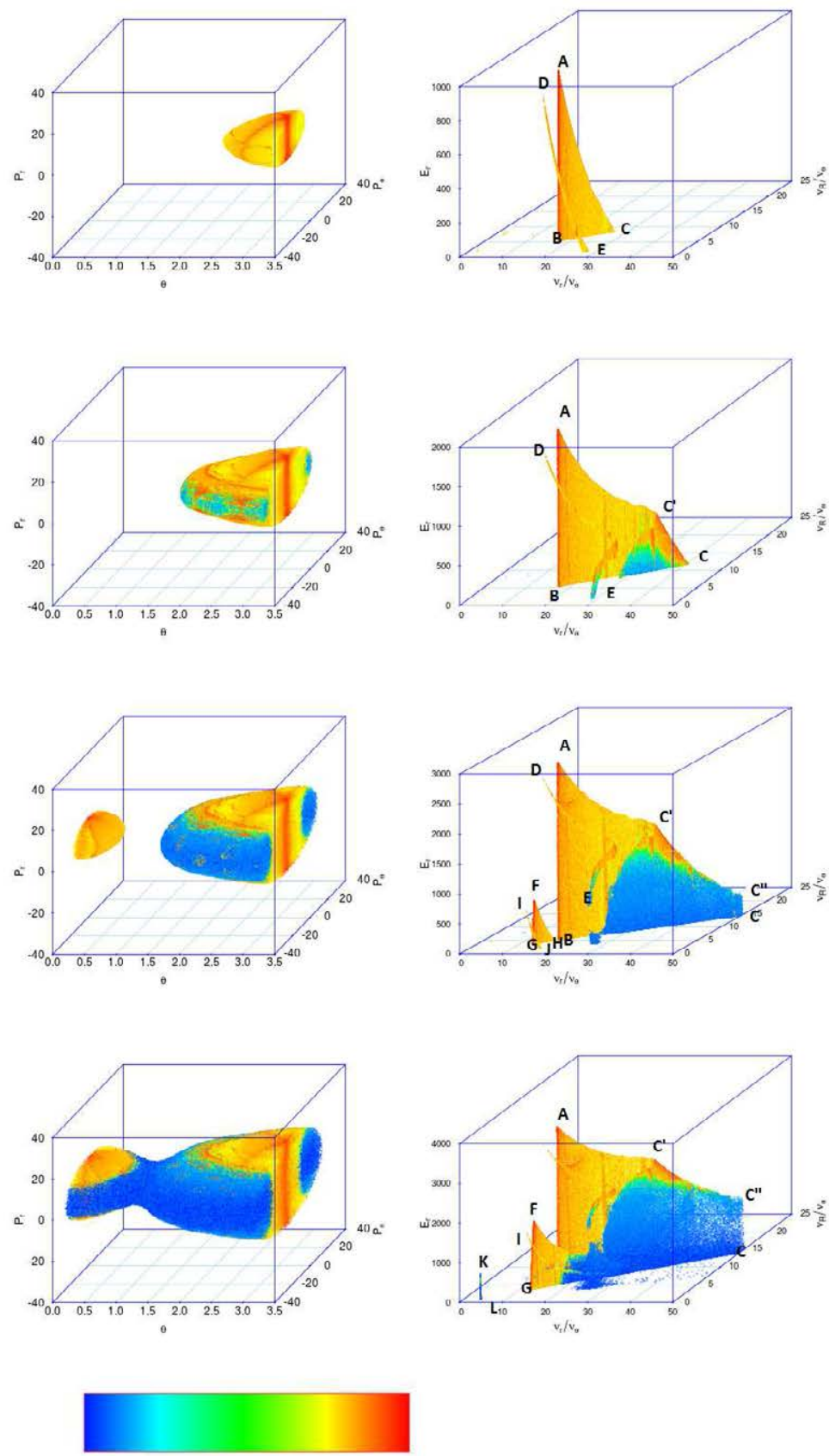

Figura 6.5: Evolución con la energía de los diagramas corrrespondientes a los mapas $S M_{3 D}$ y de frecuencia energía, MFE, coloreados con SALI para el LiNC/LiCNen 3D como hemos definido en la sección 6.1. La condiciones iniciales $z_{0} \in \Omega_{3 D}$ se han obtenido seleccionando de forma aletoria valores para $\psi, P_{\psi}$ y $P_{r}$ obteniendose el resto de los valores de acuerdo con 6.1.1. Las trayectorías han sido propagadas durante $T=7,5 \cdot 10^{5}$ u.a. para obtener los valores de $m S A L I_{T}$ en el gráfico usando la escala de colores que se incluye en la figura. 


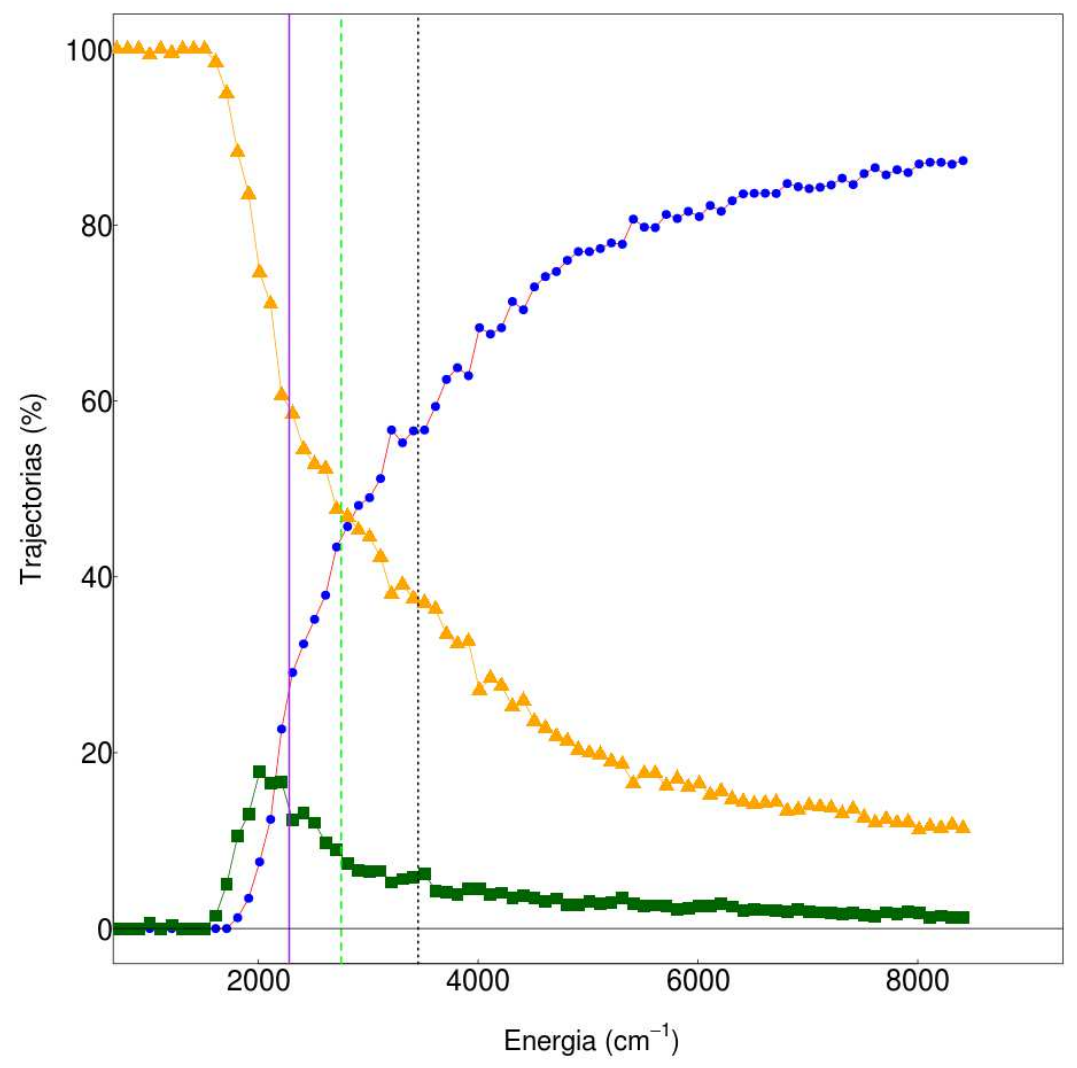

Figura 6.6: Fracción de las tres regiones del espacio de fase del LiNC/LiCN correspondientes a: Movimiento Regular (triángulos rojas) $\left(m S A L I_{T}>10^{-6}\right)$, Caos Suave (cuadrados verdes) $\left(10^{-6}>m S A L I_{T}>10^{-16}\right)$, y Caos Fuerte (círculos azules) $\left(m S A L I_{T}<10^{-16}\right)$. La línea vertical (púrpura) en $2281 \mathrm{~cm}^{-1}$ indica la energía en la que la región del pozo del isómero Li-CN comienza a ser accesible y puede existir movimiento en dicha zona, la línea vertical (verde) en $2754 \mathrm{~cm}^{-1}$ corresponde la energía inicial a la cual hay una compensación entre la destrucción de toros regulares y la aparición de nuevas regiones de regularidad. La línea vertical (negra) en $3450 \mathrm{~cm}^{-1}$ corresponde a la energía de la barrera de energi'a potencial para la reacción de isomerización.

\subsubsection{Distribución del porcentaje de caos en función de la energía}

Analizaremos en esta sección la evolución de la proporción entre las regiones de regularidad, caos suave y caos fuerte al aumentar la energía del sistema $\operatorname{LiNC} / \operatorname{LiCN}$.

Se observa que las órbitas pasan globalmente de ser más regulares a más caóticas cuando la energía global del sistema aumenta. Este efecto está cla- 
ramente ilustrado en la figura 6.6 (análoga a la figura 5.10 del modelo 2D), donde se representa el porcentaje que representan estas regiones en función de la energía de excitación del sistema. Estos porcentajes han sido obtenidos a partir de los datos de una muestra órbitas con energías comprendidas entre $1000 \mathrm{~cm}^{-1}$ y $9000 \mathrm{~cm}^{-1}$ con un intervalo de $100 \mathrm{~cm}^{-1}$ entre muestras. En total se han analizado 288.788 órbitas.

En la figura 6.6 hemos trazado tambien lineas verticales en $2281 \mathrm{~cm}^{-1}$ (energia a la que el pozo del isómero $L i-C N$ comienza a ser accesible), $2754 \mathrm{~cm}^{-1}$ (zona en la que se observa en $2 \mathrm{D}$ compensación entre la creación y la destrucción de toros regulares) y $3450 \mathrm{~cm}^{-1}$ (energía de la barrera de isomerizacion).

Para contabilizar dichos porcentajes se ha tenido en cuenta los siguientes criterios:

\section{Movimiento Regular (triángulos naranja ) $m S A L I_{T}>10^{-6}$,}

Caos suave (cuadrados verdes ) $10^{-6}>m S A L I_{T}>10^{-16}$,

Caos fuerte (círculos azules) $m S A L I_{T}<10^{-16}$.

Puede verse que a energías bajas casi todo el movimiento es regular y la fracción de la dinámica regular (triángulos naranja) se mantiene casi constante hasta una energía de $E=1550 \mathrm{~cm}^{-1}$, mostrando únicamente una disminución muy pequeña en favor de la región de caos suave.

A partir de $E>1550 \mathrm{~cm}^{-1}$ la fracción de dinámica regular cae abruptamente de forma lineal, debido a una importante destrucción de toros (teorema KAM) dando lugar al caos generalizado, hasta $E=2281 \mathrm{~cm}^{-1}$ (marcado con una línea vertical púrpura en la figura) donde la pendiente disminuye debido al hecho de que en este valor de la energía el movimiento sobre la región del segundo isómero $\mathrm{LiCN}$ es clasicamente accesible.

A diferencia del modelo $2 \mathrm{D}$, no se observa de forma global un intervalo de energías en el que se compense la creación y la destrucción de toros regulares.

Finalmente la fracción de movimiento regular continua disminuyendo, esta vez de forma exponencial hasta alcanzar un valor asintótico de $\sim 6 \%$. Resaltemos que ese valor no es cero. Esto significa que hay una zona de movimiento regular que no se destruye incluso a muy altos valores de la energía.

La conducta de la fracción de caos fuerte (círculos azules) sigue un patrón semejante al anterior, pero obviamente presentando tendencias opuestas. Esto es, hasta $E=1550 \mathrm{~cm}^{-1}$ el porcentaje de movimiento caótico permanece nulo y en ese punto crece súbitamente de forma lineal hasta $E=2281 \mathrm{~cm}^{-1}$, 
valor de la energía donde la pendiente disminuye un poco, y finalmente esta magnitud crece hasta alcanzar el valor asintótico de $\sim 93 \%$.

Por otra parte, la fracción de caos suave (cuadrados verdes) se comporta de forma muy diferente al caos fuerte, presentando características bastante interesantes.

Se observa que el caos suave solo existe significativamente en el intervalo $E=1600-2900 \mathrm{~cm}^{-1}$. Se observa igualmente que el caos suave comienza a crecer significativamente a un valor ligeramente inferior de energía que el caos fuerte, una vez alcanzado un máximo del $21.08 \%$ en $E=1850 \mathrm{~cm}^{-1}$, cae rápidamente a un valor pequeño manteniendo un valor residual del $\sim 0,6 \%$ entre $E=2900-9000 \mathrm{~cm}^{-1}$. Esta es la región de la influencia del cantoro, que atrapa temporalmente trayectorias dentro de él, que luego se mantienen cerca de la frontera de regularidad del isómero LiNC que supone un valor más bajo de $m S A L I_{T}$, y por tanto se detecta caos suave.

A medida que la energía aumenta, el cantoro se destruye progresivamente, debilitando su papel de barrera para el flujo de trayectorias a su través.

En consecuencia, el efecto que hemos discutido se vuelve progresivamente menos importante, haciendo que la fracción de caos suave tienda a ser despreciable, hasta que solo el caos fuerte sea predominante en la región ergódica del espacio de fase.

\subsection{Comparación entre los modelos 2D y 3D}

En esta sección compararemos los resultados obtenidos en el modelo utilizado en el capitulo anterior con el modelo 2D. Se comprueba (véase la figura 6.7) que los resultados obtenidos en $2 \mathrm{D}$ son muy parecidos a los obtenidos en $3 \mathrm{D}$ cuando se asigna el valor de equilibro $r=2,186$ u.a., $P_{r}=0$ u.a. a las coordenadas $r$ y $P_{r}$.

Para constrastar esta afirmación del modelo $3 D$, hemos calculado seis distribuciones del porcentaje de orbitas caóticas para energı'as comprendidas en el intervalo $[1500,3900] \mathrm{cm}^{-1}$ con un valor fijo de $P_{r}$. El resultado se muestra en la figura 6.8 en la que distinguimos los diferentes valores del valor inicial de $P_{r}$ mediante el uso de diferentes colores.

Se puede comprobar que cuando $P_{r}=0$ la distribución de la energía es muy similar a la observada en el modelo 2D. Cuando $P_{r}$ tiene un valor distinto de 0 la distribución se desplaza a la derecha, observandose la zona en en la que se equilibran la creación y la destrucción de toros regulares entre las lineas verde y negra.

Si comparamos los valores de dichas curvas con la distribucion total de 

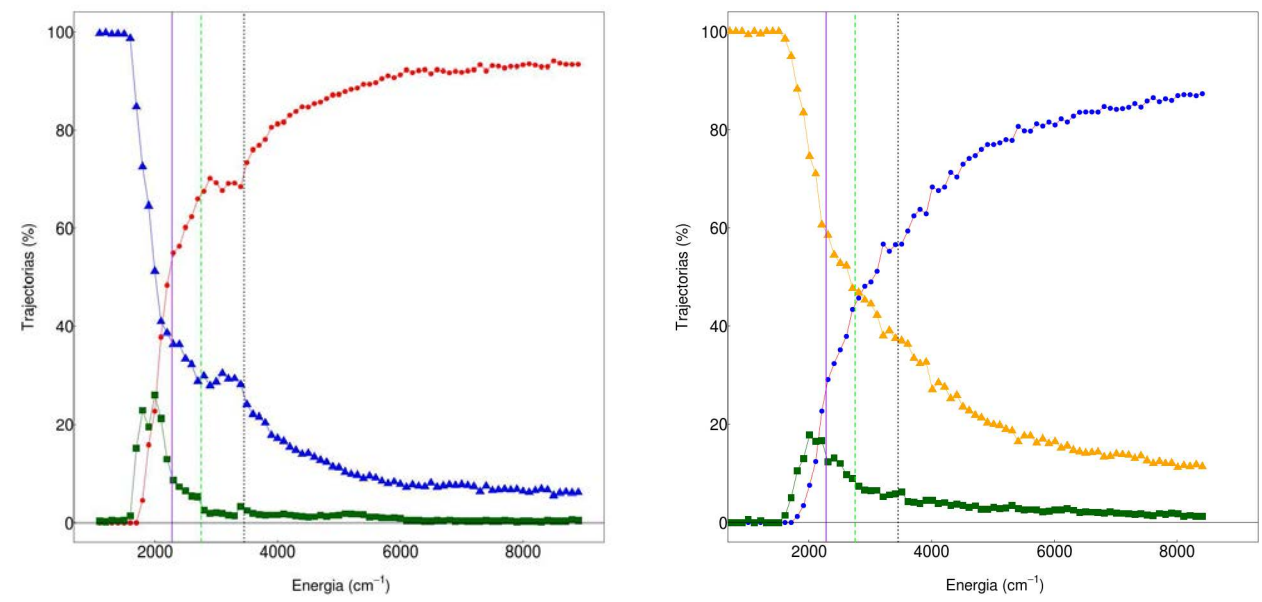

Figura 6.7: Comparación entre la distribución de orbitas caóticas en 2D (izquierda) y 3D (derecha)

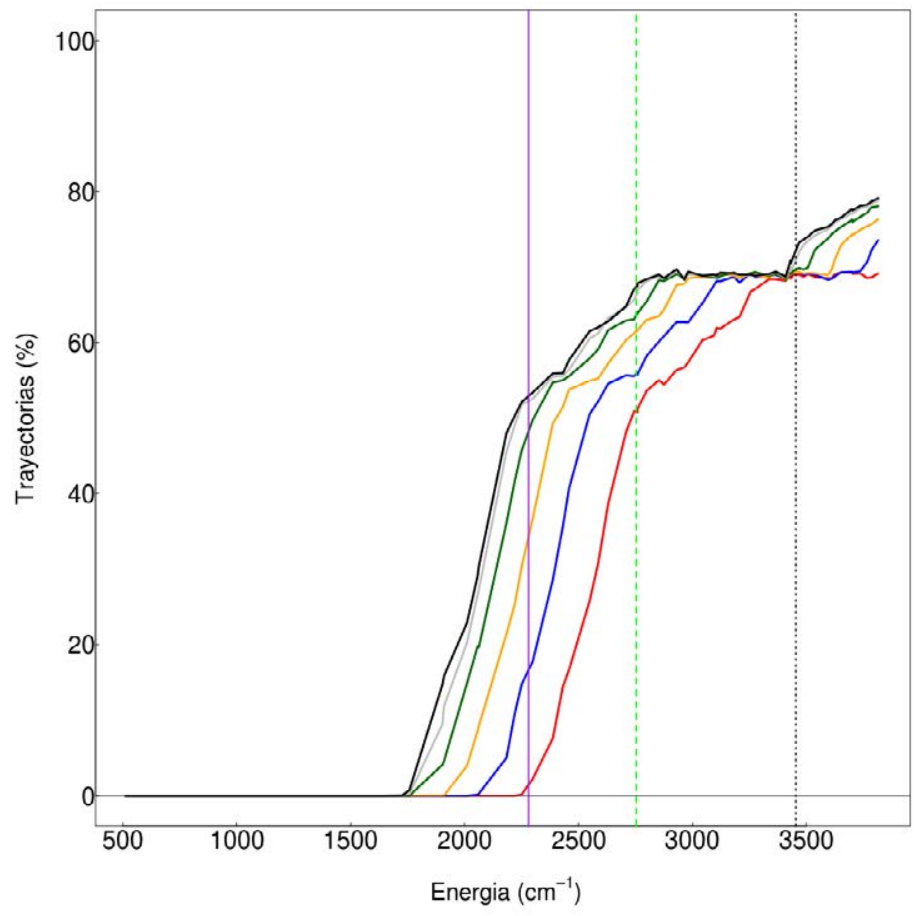

Figura 6.8: Porcentaje de órbitas caóticas en 3D en función de la energía y del valor de $P_{r}$ 
los porcentajes de caos en 3D (figura 6.8 ) vemos que esta última presenta una curva mas suavizada relativamente diferente de la distribución que se observa en $2 \mathrm{D}$ en la que en particular no se observa la zona de equilibrio entre la lineas verde y purpura. Claramente esto es debido al hecho de que se promedian los valores correspondientes a varios valores de $P_{r}$, la distribución de las órbitas caóticas es practicamente equivalente a la que se ha observado en el modelo 2D. Por tanto el porcentaje de caos no depende de la dimensión del sistema sino de la energía disponible por las coordenadas $R$ y $\theta$

Se puede observar que el efecto de asignar valores a $P_{r}$, o equivalentemente dotar de alguna energía cinética a la coordenada $r$, implica la reducción de la energía disponible para las otras coordenadas. Esto se puede explicar por la pequeñez del factor de acoplamiento $\frac{P_{\theta}^{2} r^{-3}}{m_{2}^{2}}$ que aparece en el termino de energía cinéteca del resultado (ver sección 5.1, como ya se ha explicado en esta sección un factor de acoplmiento muy pequeño implica el que la coordenada $r$ actue de forma practicamente independiente y por tanto que la energia suministrada a la vibración de $r$ no fluye a las otras coordenadas del sistema molecular $\operatorname{LiNC} / \operatorname{LiCN}$. 


\subsection{SUMARIO}

De la observación de los resultados que presentan los distintos mapas estudiados se concluye que el espacio de fase que posee el $\operatorname{LiNC} / \mathrm{LiCN}$ en 3D se puede dividir basicamente en las mismas tres regiones que ya se observaban en $2 \mathrm{D}$.

- Región de Movimiento Regular: Compuesta por trayectorias que están organizadas en toros KAM alrededor de los pozos de potencial correspondientes a los dos isómeros. El tamaño de la zona regular esta correlacionado con la energía disponible por el sistema (es máximo en $1000 \mathrm{~cm}^{-1}$ y mínimo en 4000. Por otra parte se observa como el número de orbitas regular se incrementa con el valor absoluto de $P_{r}$, dado que que la energía disponible a los otros modos de vibración es menor mostrando que el movimiento en la coordenada angular es la principal causa de caos en este sistema..

- Región de Caos Suave: Compuesta por trayectorias irregulares que están influenciadas por la frontera de las regiones regulares mencionadas antes y otras estructuras casi regulares, como los cantoros.

- Región de Caos Fuerte: Compuesta por trayectorias caóticas que se mueven libremente, recorriendo la parte ergódica del espacio de fasea accesible a cada energía.

Al representar las órbitas en el espacio de frecuencias, se comprueba que las orbitas regulares se agrupan a lo largo de lineas de resonancia. A medida de que la energía crece, crece también el número de lineas de resonancia accesibles. Se comprueba que las orbitas caóticas se sitúan mayoritariamente en el entorno de la intersección de lineas de resonancia. Ademas se pone de manifiesto que el movimiento de las trayectorias caóticas no es completamente érgodico sino que éste está influenciado por las resonancias que hacen que las trayectorias caóticas pasen periodos de tiempo atraidas o atrapadas por las mismas.

Por otra parte, al representar las orbitas en el espacio de energias se comprueba que las órbitas regulares se agrupan en figuras planas relacionadas con los pozos, mientras que las orbitas caóticas se esparcen por el espacio de energía. 


\section{Capítulo 7}

\section{Estudio detallado del $L i N C / L i C N$ a baja energía}

En este capítulo usaremos los mapas 3D desarrollados en el capítulo anterior para estudiar el sistema molécular $L i N C / L i C N$ a la energía vibracional de $512 \mathrm{~cm}^{-1}$. Resaltamos que se trata de una energía muy baja, de hecho ligeramente inferior a la energía del nivel cuántico fundamental de la molecula.

El trabajar con una energía baja facilita cierta simplificación del sistema puesto que no existe la presencia de las orbitas caóticas (recordemos que según los resultados del capítulo anterior no se detecta caos cuando el sistema está por debajo de la energía vibracional de $1550 \mathrm{~cm}^{-1}$ ), por otra parte, a este nivel de energía el hamiltoniano se puede reducir a uno casi integrable.

Dedicaremos la primera sección de este capítulo (7.1) a la discusión de los resultados obtenidos utilizando los mapas $S M_{3 D}, M E M_{3 D}$ y $M F E_{3 D}$ calculados a esta energía. A continuación procederemos a clasificar las órbitas en grupos característicos que discutiremos en la seección (7.2), terminaremos el capítulo el resumen en la sección (7.3).

\subsection{Mapas 3D y Visualización 4D}

Para generar los mapas en 3D a esta energía, hemos seleccionado previamente un númeroso grupo de condiciones iniciales de acuerdo con la estrategia que definimos en el capítulo anterior.

Concretamente se han selecionado 700.794 condiciones iniciales escogidas de acuerdo con la siguiente metodología.

- $P_{r}$ se elige al azar entre sus valores mínimo y máximo ,-7.40 u.a y 7.40 
u.a respectivamente mientras que $r$ se iguala a su valor de equilibrio 2,186 u.a.

- Coordenadas $\theta$ y $P_{\theta}$ escogidas al azar.

- $R$ se escoge en función de $\theta$ para que la órbitas se encuentren en el camino de mínima energía de acuerdo con la ecuación (4.7), mientras que $P_{R}$ se escoge para cumplir la condición de que las órbitas tengan la energía de $512 \mathrm{~cm}^{-1}$.

A dichas órbitas se las deja evolucionar durante $2,510^{5}$ u.a de tiempo.

El resultado se muestra en los diagramas de la figura 7.1, que corresonde a la visualización $4 \mathrm{D}$ de los mapas correspondientes en los que el valor de $m S A L I_{T}$ se representa mediante un código de colores.
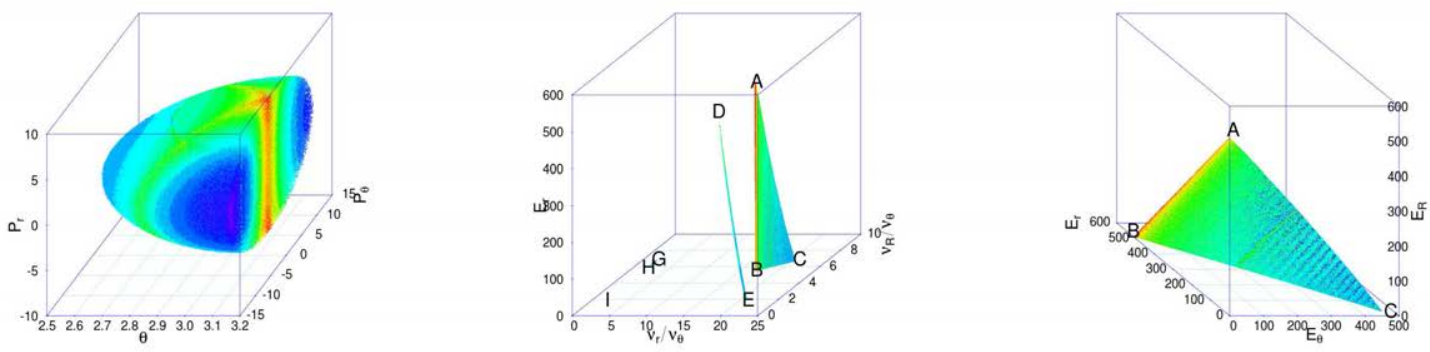

Figura 7.1: Visualizaciones $4 \mathrm{D}$ de los mapas $S M_{3 D}$ (izquierda) $M F E_{3 D}$ (centro) y $M E M_{3 D}$ (derecha) a la energía de $512 \mathrm{~cm}^{-1}$. El color indica el valor de $m S A L I_{T}$.

\subsubsection{Mapa $S M_{3 D}$}

Como se observa en la figura 7.2 las órbitas estudiadas se agrupan en el interior de un semielipsoide de ejes $\psi \in[2,5, \pi] \mathrm{rad}, P_{r} \in[-10,10]$ u.a y $P_{\psi} \in[-15,15]$ u.a. Para describir la estructura interior de dicho semielipsoide se han calculado los cortes del diagrama $3 \mathrm{D}$ del mapa $S M_{3 D}$ con los planos definidos por tener un valor de $P_{r}$.

En el entorno de los puntos cercanos a la recta perpendicular al plano $\left(\psi, P_{\psi}\right)$ que pasa por el pozo de potencial $\psi=\pi$ se observa la existencia de una zona cuyo alto valor de $m S A L I_{T}$ evidencia una alta estabilidad. Se observa que, en general, el valor de $m S A L I_{T}$ es mínimo para las órbitas con 

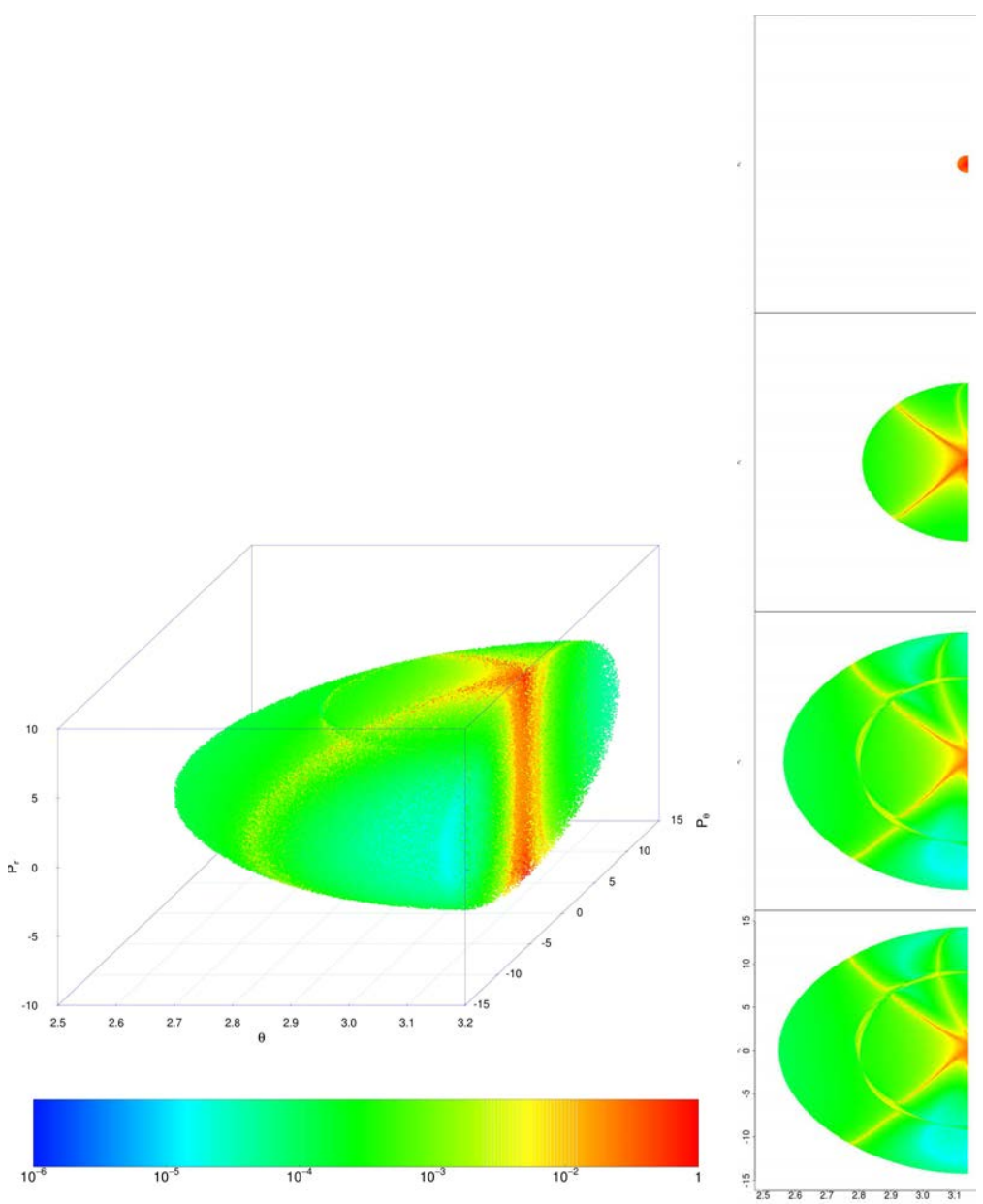

Figura 7.2: Visualización del mapa $S M_{3 D}$ en 3D a la energía de $512,0 \mathrm{~cm}^{-1}$ en 3D (izquierda) y cortes del mismo para distintos valores de $P_{r}$ (derecha). El valor de $-\log _{10}\left(m S A L I_{t}\right.$ se muestra con un codigo de colores incluido en la figura

valores valores máximos del $\left|P_{\theta}\right|$, existiendo otra zona relativamente inestable que coincide con los valores bajos de $\psi$.

En los cortes con los planos con un valor definido de $P_{r}$, se observa la existencia de dos zonas con un alto valor de $m S A L I_{T}$ que parten del pozo del potencial $\psi=\pi$ hacia la periferia. Dichas zonas coinciden aproximadamnte con las definidas por las rectas $P_{\theta}=0$. 


\subsection{2. $\quad$ Mapa $M F E_{3 D}$}
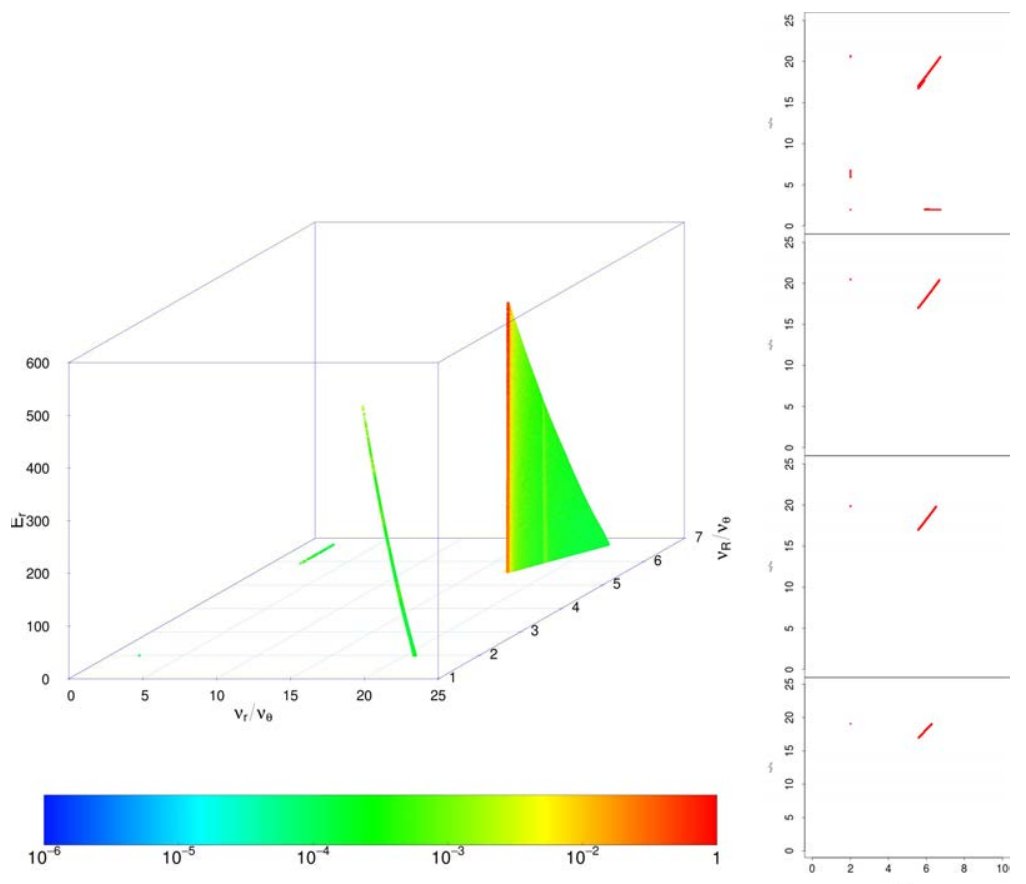

Figura 7.3: Visualización 4D del diagrama correspondiente al mapa $M F E_{3 D}$ (izquierda) y cortes del mismo con planos con un valor de $E r$ definido (derecha).

En el diagrama correspondiente al mapa $M F E_{3 D}$ mostrado en la figura 7.3 se representan las relaciones de frecuencia de las orbitas estudiadas frente a la energía media de $r$. En dicha figura se aprecia un triángulo $A B C$ donde se agrupan la mayoria de las órbitas. Dicho triángulo se caracteriza pr tener la relació de frecuencias $(2,-6,1)$ entre las orbitas. Se distingue asimismo dos lineas aisladas $\overline{C D}$ y $\overline{E F}$ y un punto aislado $G$ que corresponden a órbitas que tienen las relaciones de resonancia $(1,0,-2),(0,1,-2)$ y $(2,2,-1)$ respectivamente. 
7.1.3. Mapa $M E M_{3 D}$

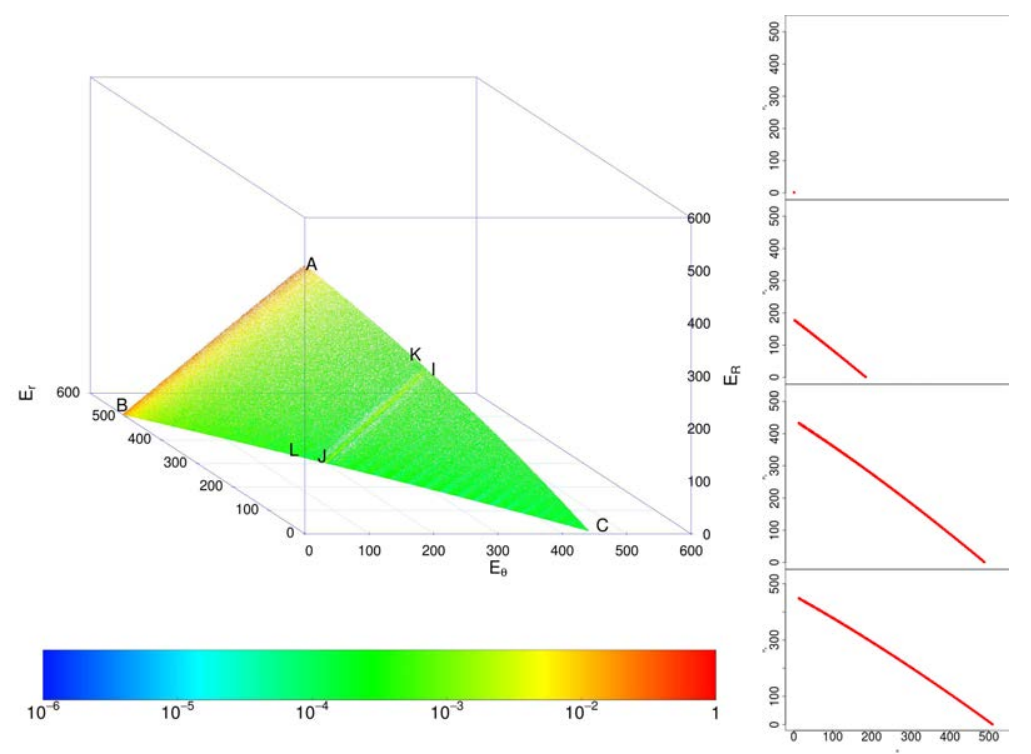

Figura 7.4: Mapa de energía cinética media de las órbitas estudiadas a $512 \mathrm{~cm}^{-1}$ (izquierda). Cortes del diagrama $3 \mathrm{D}$ del mapa $M E M_{3 D}$ con planos con un valor de $E r$ definido (derecha).

Representamos en la figura (7.4) los resultados correspondientes al mapa $M E M_{3 D}$ calculados a la energía de $512 \mathrm{~cm}^{-1}$. Se observa que las orbitas se agrupan en el triángulo plano $A B C$ de lados aproximadamente rectilíneos que cortan a los planos $\left(E_{R}, E_{\theta}\right),\left(E_{r}, E_{\theta}\right)\left(E_{r} . E_{r}\right)$ en la parte positiva formando un triangulo aproximadamente equilatero. Los lados del triángulo citado $\overline{A B}, \overline{A C}$ y $\overline{B C}$, agrupan a las orbitas que presenta un nivel de energía cinética media muy bajo en uno de los modos de vibracion. Mientras que Los vertices del triángulo $A, B, C$ corresponden a órbitas en las que la energía cinética se atribuye exclusivamente a una de las coordenadas.

Se puede determinar que los valores máximos de $m S A L I_{T}$ se encuentran situados en el entorno de la recta $\overline{A B}$ disminuyendo a medida que nos desplazamos hacia el punto $C$.

Por otra parte destacamos una zona con valores altos de $m S A L I_{T}$ que se situa en una linea $\overline{A^{\prime} B^{\prime}}$ paralela a la linea $\overline{A B}$. Se verificara posteriormente que se trata de órbitas que presentan una relación de resonancia $(1,0,-6)$. 


\subsection{Clasificación de las órbitas estudiadas}
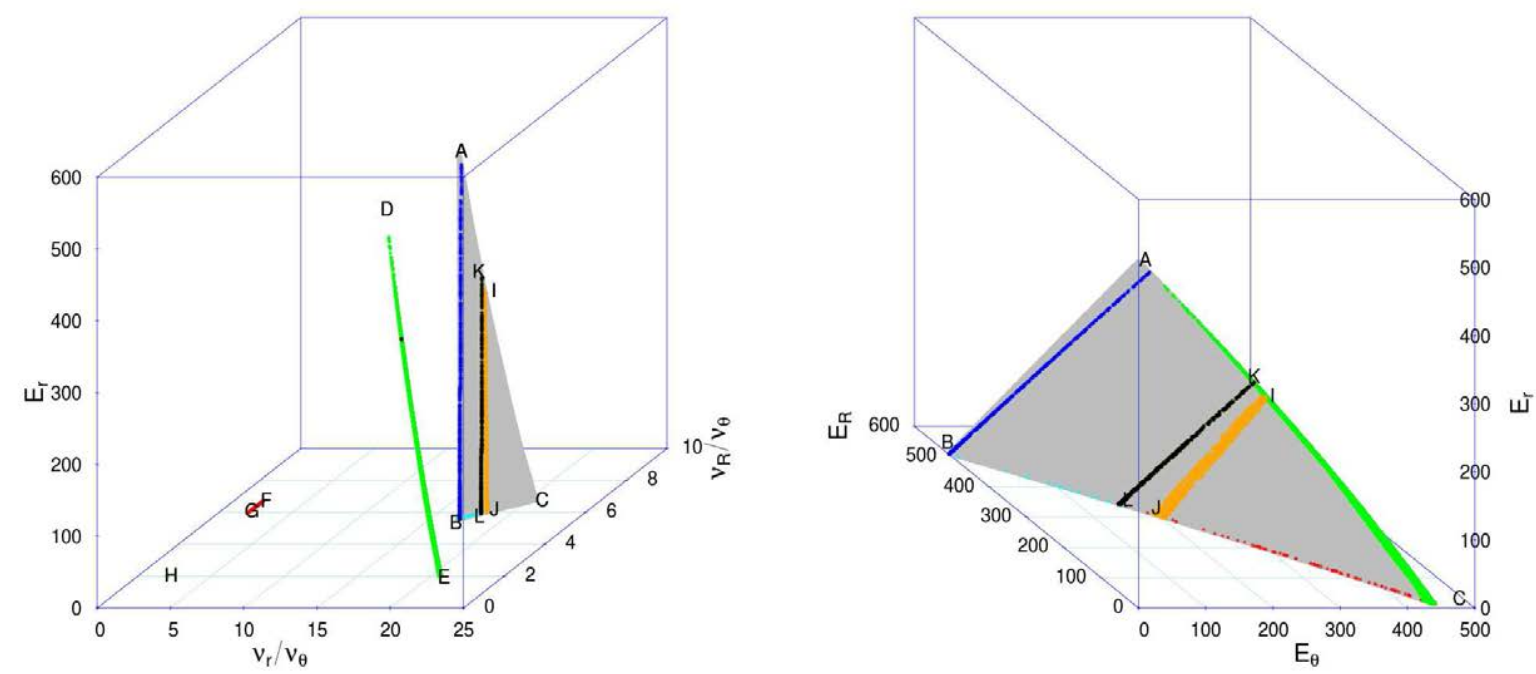

Figura 7.5: Clasificación de las órbitas estudiadas en el mapa $M F E_{3 D}$ (izquierda) y en el mapa $M E M_{3 D}$ (derecha), véase la tabla 7.1.

Para clasificar las órbitas estudiadas vamos a considerar simultaneamente la información suministrada por los mapas $M E M_{3 D}$ y $M F E_{3 D}$ que se presenta en la figura (7.5).

Consideremos el triángulo $A B C$ del mapa $M F E_{3 D}$ al que le hemos asignado el color gris en la figura 7.5, (izquierda ). Las orbitas que forman parte de este grupo son la práctica totalidad de las estudiadas en esta energía ( $98 \%$ del total de la muestra). Se observa que las frecuencias de sus modos de vibración estan en la relación de resonancia $(2,-6,1)$. El triángulo $A B C$ citado se corresponde con el interior del triangulo $A B C$ del mapa $M E M_{3 D}$ (figura 7.5, a la derecha), por tanto las órbitas de consideradas tienen energía cinética en todos los modos de vibración. Denominaremos dicho grupo de órbitas órbitas en posición general .

Consideremos ahora las órbitas correspondientes a la relación de frecuencias $(0,1,-2)$ que se representan en el segmento $\overline{F G}$ del mapa $M F E_{3 D}$. Dicho grupo mucho menos numeroso se sitúa en el lado $\overline{A C}$ del mapa $M E M_{3 D}$. Se trata por tanto de un grupo de órbitas que carece practicamente de energía cinética en el modo de vibración $r$.

Consideremos las órbitas en la relación de frecuencias $(1,-3,0)$ que se 
representan como el segmento $\overline{B L}$ en el mapa $M F E_{3 D}$. Como en el caso anterior se sitúan tambien en el lado $\overline{B C}$ y por tanto tiene muy poca energía cinética en $r$.

Las órbitas con poca energía en $r$ pueden ser por tanto de tres tipos: pueden estar en posición general, tener la relación de resonancía $(0,1,-2)$ o bien la relación $(1,-3,0)$.

Continuando con nuestro análisis consideramos las órbitas que cumplan relación de resonancia $(1,0,-2)$ que se representa como la recta $\overline{D E}$ del mapa $M F E_{3 D}$. En el mapa $M E M_{3 D}$ dichas órbitas se situan en el lado $\overline{A C}$, por tanto se trata de orbitas sin energía potencial en el modo de vibración $R$. Como en el caso anterior, observamos que las orbitas con poca energía en cinética en el modo de vibración $R$ pueden estar en posición general, o tener la relación de resonancia $(1,0,-2)$.

Observaremos ahora a las orbitas en la relación de resonancia $(0,1,-17)$ que se situan en las cercanias de la recta $\overline{A B}$ del mapa $M F E_{3 D}$. Dichas órbitas se situan en la recta $\overline{A B}$ del mapa $M E M_{3 D}$. Se trata por tanto de orbitas con muy poca energía cinética en $\theta$.

Consideremos ahora a la relación $(1,0,-6)$ que se situa en las cercanias de la recta $\overline{I J}$. Se trata de una resonancia muy fuerte que se manifiesta tambien como una cadena de islas en el modelo 2D. En el mapa $M E M_{3 D}$ se observa análogamente como se distribuyen en el entorno de la recta $\overline{I J}$. Una relaci ón muy parecida se puede establecer cambiando los papeles de $R$ y $r$ en la relacíon $(0,1,-18)$. Se observa que las orbitas relacionadas con esta última resonancia, se situan el el entorno de la recta $\overline{K L}$.

Finalmente se observa en el mapa $M F E_{3 D}$ el punto $H$ que corresponde a una unica órbita aislada con relación de frecuencias $(2,2,-1)$. Dicha orbita esta muy cerca del punto $C$ del mapa $M E M_{3 D}$.

Resumimos en la tabla 7.1 la información sobre los distintos grupos de órbitas que hemos considerado. 
106CAPÍTULO 7. ESTUDIO DETALLADO DEL LINC/LICN A BAJA ENERGÍA

Tabla 7.1: Grupos de órbitas seleccionadas a $512,0 \mathrm{~cm}^{-1}$ en la figura 7.5

Grupo. Resonancia Mapa $M F E_{3 D}$ Mapa $M E M_{3 D}$

\begin{tabular}{llll}
\hline (a) & $(0,1,-2)$ & Segmento $\overline{F G}$ & Segmento $\overline{L C}$ \\
(b) & $(1,-3,0)$ & Segmento $\overline{B L}$ & Segmento $\overline{B L}$ \\
(c) & $(0,1,-17)$ & Segmento $\overline{A B}$ & Segmento $\overline{A B}$ \\
(d) & $(1,0,-2)$ & Segmento $\overline{D E}$ & Segmento $\overline{A C}$ \\
(e) & $(1,0,-6)$ & Segmento $\overline{I J}$ & Segmento $\overline{I J}$ \\
(f) & $(0,1,-18)$ & Segmento $\overline{K L}$ & Segmento $\overline{K L}$ \\
(g) & $(2,-6,1)$ & Triángulo $A B C$ & Triángulo $A B C$ \\
(h) & $(2,2,-1)$ & Punto $H$ & Punto $C$ \\
\hline
\end{tabular}


En las secciones siguientes se presenta una descripción detallada de las órbitas correspondientes a cada uno de los grupos mostrados en la tabla 7.1.

En particular se presentamos para cada órbita los siguientes paneles:

- (a): Plano $\left(\psi, P_{\psi}\right)$ del corte del mapa $S M_{3 D}$ con el plano $P_{r}=P_{r}(0)$, siendo $P_{r}(0)$ el valor inicial de $P_{r}$, la órbita se representa con un cuadrado negro.

- (b): Plano $\left(\nu_{r} / \nu_{\theta}, \nu_{R} / \nu_{t}\right.$ heta $)$, la órbita se representa con un cuadrado negro.

- (c): Diagrama 3D del mapa $M E M_{3 D}$, la órbita se representa con un cuadrado negro.

- (d): Evolución de $S A L I$ en función del tiempo.

- (e): Representación de la órbita en el espacio de configuración.

- (f): Representación de la órbita en el espacio de los momentos.

- (g): Proyección de la órbita en el plano $(R, \psi)$.

- (h): Proyección de la órbita en el plano $(r, \psi)$.

- (i): Proyección de la órbita en el plano $(R, r)$.

- (j): Proyección de la órbita en el plano $\left(R, P_{R}\right)$.

- (k): Proyección de la órbita en el plano $\left(r, P_{r}\right)$.

- (l): Proyección de la órbita en el plano $\left(\psi, P_{\psi}\right)$.

- (m): Evolucion de $R$ en función del tiempo.

- (n): Evolucion de $r$ en función del tiempo.

- ( $\tilde{n})$ : Evolucion de $\psi$ en función del tiempo. 


\subsection{1. Órbitas en la resonancia $(0,1,-2)$}
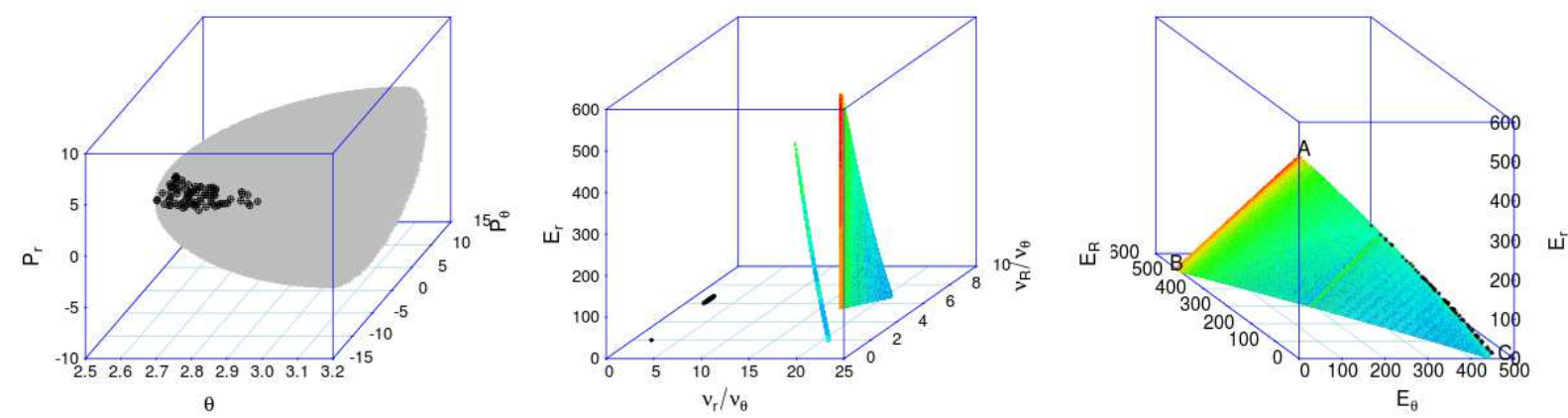

Figura 7.6: Grupo (a). Órbitas ligadas a la resonancia $(0,1,-2)$ entre $r$ y $\theta$. Se muestra la posición de las órbitas con puntos negros en los distintos mapas: $S M_{3 D}$ (izquierda), $M F E_{3 D}$ (centro) y $M E M_{3 D}$ (derecha).

Analizaremos en este grupo las órbitas cuya relación de frecuencias es $(0,1,-2)$. Dichas órbitas se agrupan en el segmento $\overline{J C}$ de los mapas $M F E_{3 D}$ y $M E M_{3 D}$. En el mapa $S M_{3 D}$ se observa que las órbitas (representadas como puntos negros en el diagrama 3D del mapa) se agrupan como una nube de puntos en el plano $P_{r}=0$ en el intervalo $\theta \in[2,5,2,8] \mathrm{rad}$.

Representamos en las figuras 7.7,7.8 y 7.9 ejemplos concretos de órbitas con esta relación de resonancia.

Se puede comprobar (panel (b)) que la relación de resonancia de estas órbitas es efectivamente $(1,0,-6)$ y que las mismas se encuentran en el segmento $\overline{J C}$ del diagrama correspondiente al mapa $M E M_{3 D}$ (panel (c)).

El indicador $S A L I$ muestra que las orbitas escogidos son estables,panel (d). Esto significa que debe estar inmersas en un toro.

Se observa en el corte con el diagrama del mapa $S M_{3 D}$ (panel (a)) que las órbitas escogidas se encuentran en el interior del corte, relativamente lejos del pozo.

Podemos comprobar en el panel(k) que la proyección de las órbitas escogidas en el plano $\left(r, P_{r}\right)$ se reduce a un punto. Esto implica que $E_{r}=0$ (ver A y por tanto que la orbita solo vibra en $\operatorname{los} \operatorname{modos} R$ y $\theta$. Se trata por tanto de un 2-toro. 
(a)

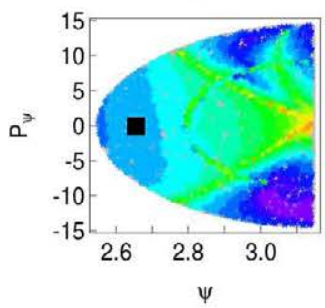

(d)

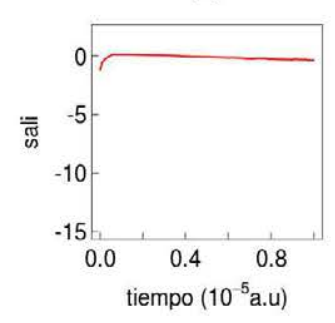

(g)

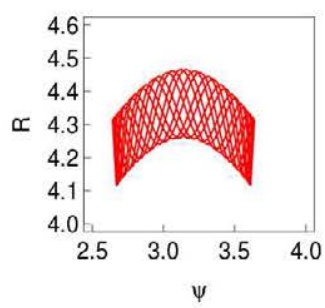

(j)

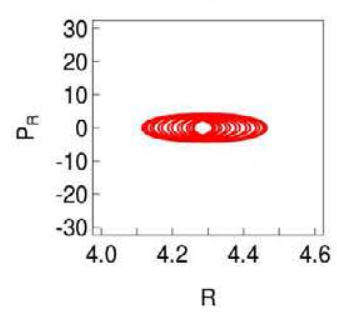

(m)

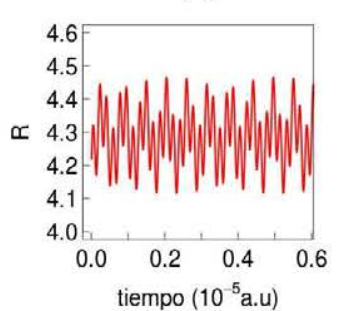

(b)

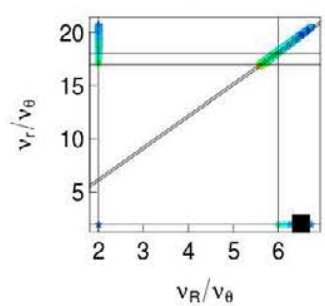

(e)

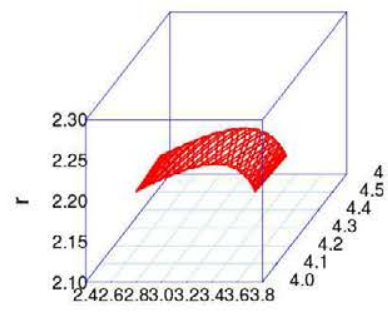

(h)

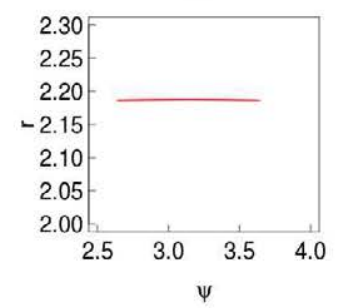

(k)

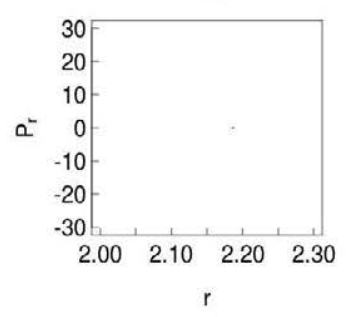

(n)

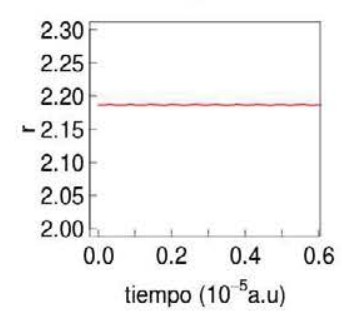

(c)

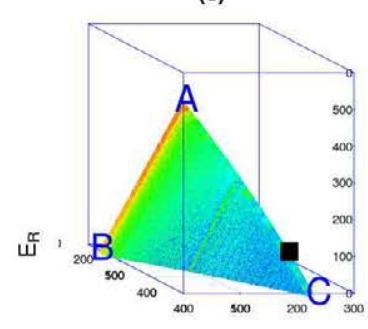

(f) $E_{\theta}$

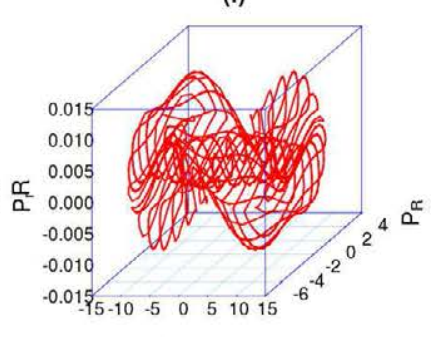

$\mathrm{P}_{\theta}$

(i)

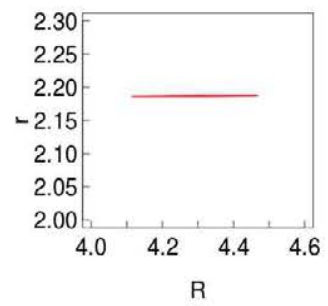

I

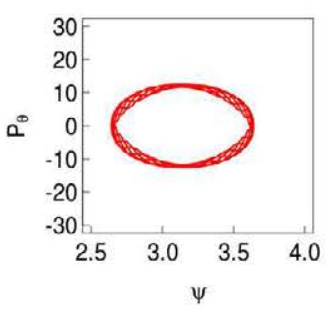

(立)

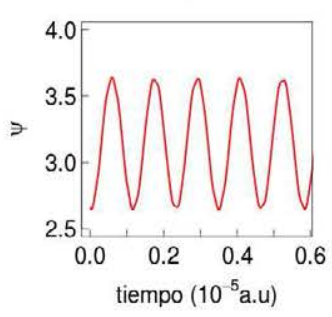

Figura 7.7: Descripción detallada de la órbita a1. 
(a)

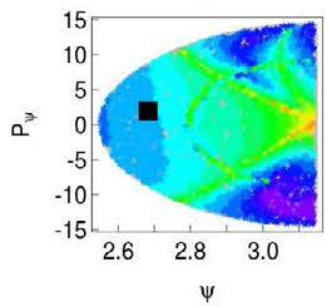

(d)

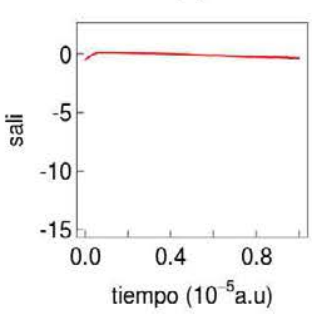

(g)

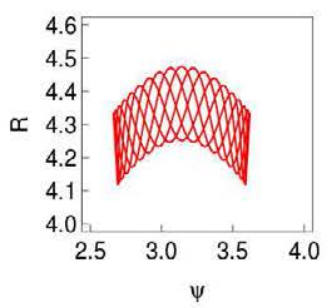

(j)

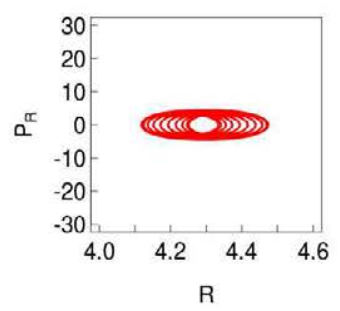

(m)

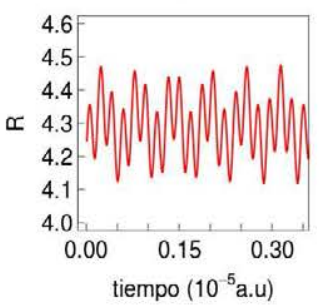

(b)

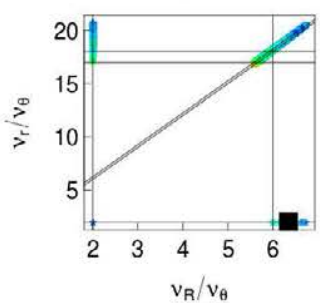

(e)

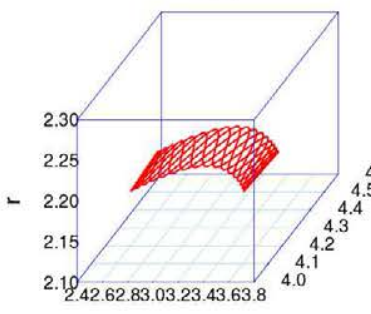

(h)

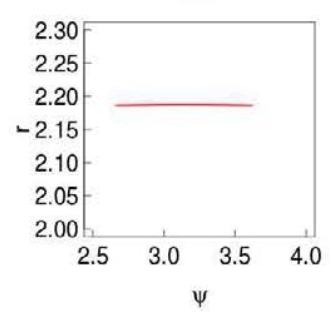

(k)

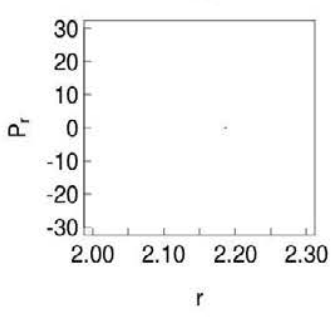

(n)

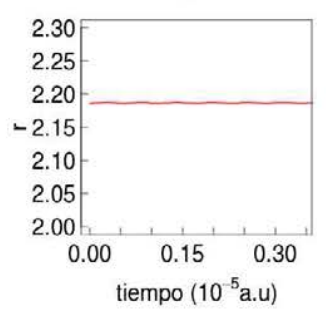

(c)

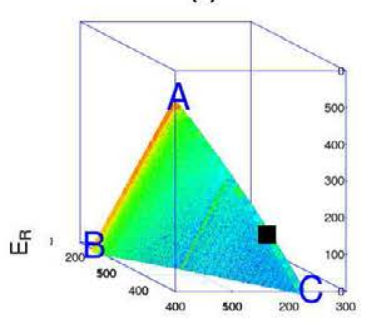

(f) $E_{\theta}$

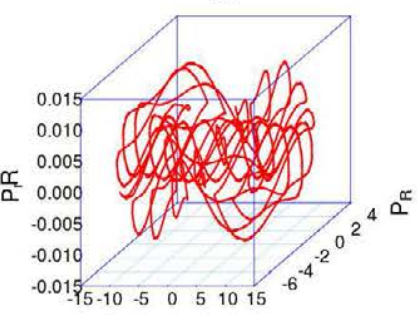

$\mathrm{P}_{\theta}$

(i)

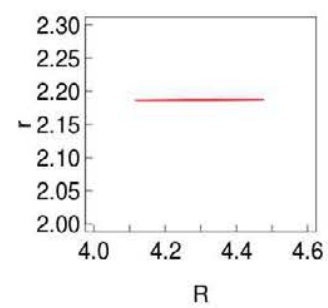

I

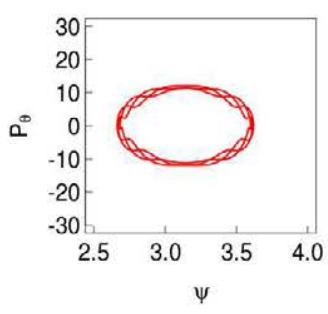

(n)

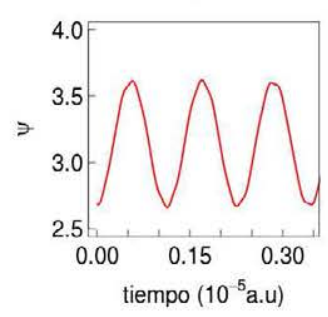

Figura 7.8: Descripción de la órbita a2. 
(a)

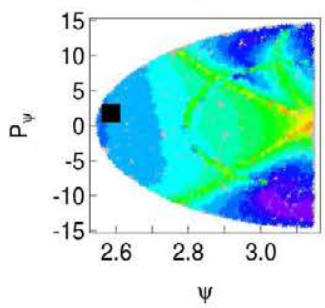

(d)

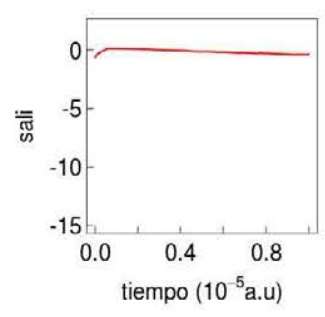

(g)

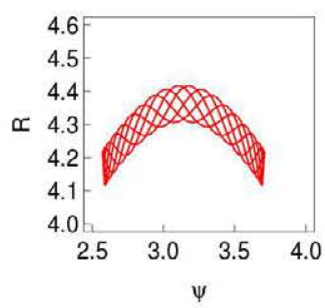

(j)

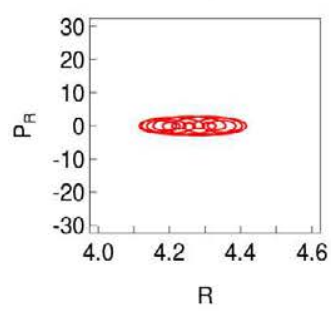

(m)

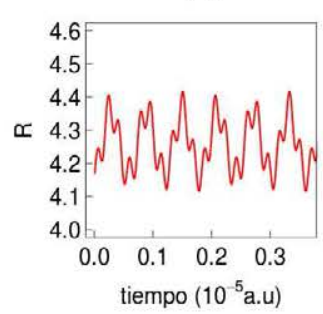

(b)

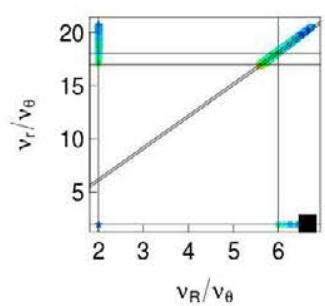

(e)

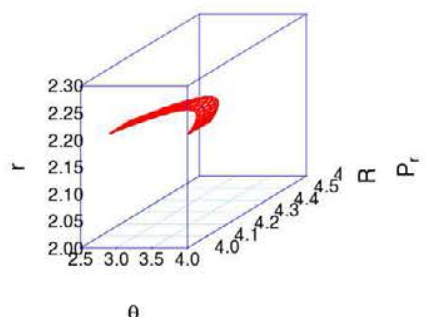

(h)

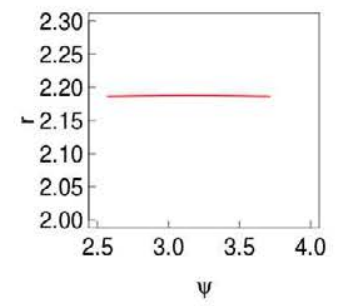

(k)

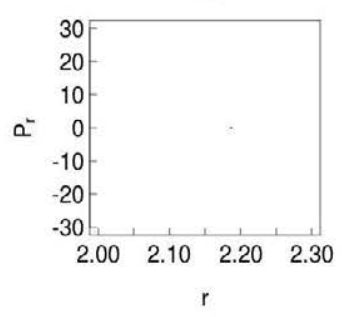

(n)

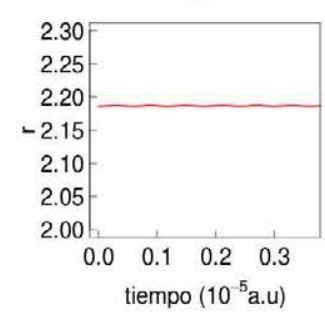

(c)

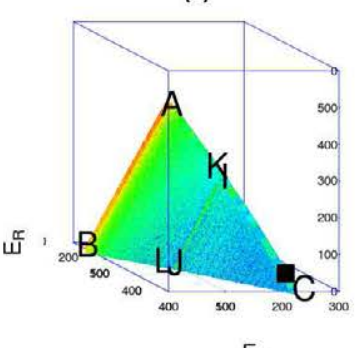

(f)

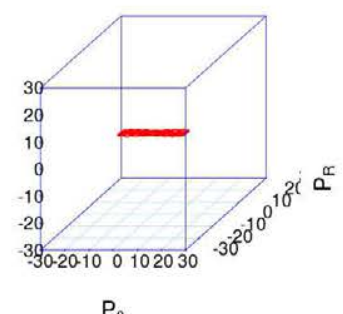

(i)

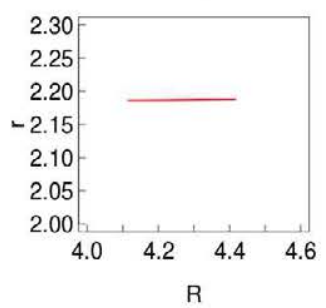

I

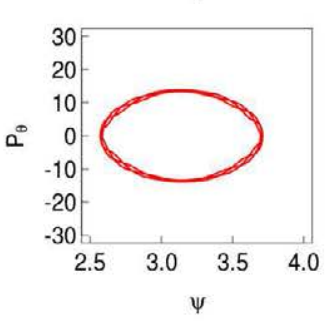

(立)

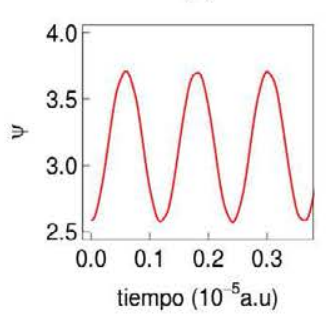

Figura 7.9: Descripción de la órbita a3. 


\subsection{2. Órbitas en la resonancia $(1,-3,0)$}
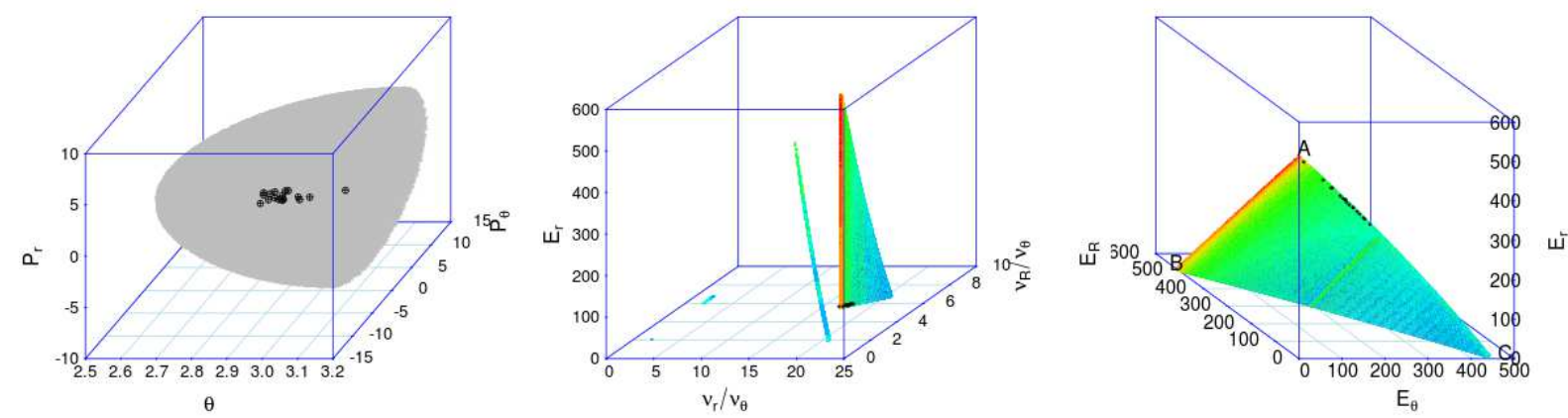

Figura 7.10: Grupo (b). Órbitas ligadas a la resonancia $(1,-3,0)$ entre $R$ y $r$. Se muestra la posición de las órbitas con puntos negros en los distintos mapas: $S M_{3 D}$ (izquierda), $M F E_{3 D}$ (centro) y $M E M_{3 D}$ (derecha).

Analizaremos en este grupo las órbitas cuya relación de frecuencias es $(1,-3,0)$. Dichas órbitas se agrupan en el segmento $\overline{F G}$ del mapa $M F E_{3 D}$ y el segmento $\overline{B L}$ del mapa $M E M_{3 D}$. En el mapa $S M_{3 D}$ se observa que las órbitas (representadas como puntos negros en el diagrama 3D del mapa) se agrupan como una nube de puntos en el plano $P_{r}=0$ en el intervalo $\psi \in[2,8, \pi] \mathrm{rad}$.

Representamos en las figuras 7.11,7.12 y 7.13 ejemplos concretos de órbitas con esta relación de resonancia.

Se puede comprobar (panel (b)) que la relación de resonancia de estas órbitas es efectivamente $(1,-3,0)$ y que las mismas se encuentran en el segmento $\overline{B L}$ del diagrama correspondiente al mapa $M E M_{3 D}$ (panel (c)).

El indicador $S A L I$ muestra que las orbitas escogidos son estables,panel (d). Esto significa que debe estar inmersas en un toro.

Se observa en el corte con el diagrama del mapa $S M_{3 D}$ (panel (a)) que las órbitas escogidas se encuentran en el interior del corte, relativamente lejos del pozo.

Como en el caso anterior, podemos comprobar en el panel(k) que la proyección de las órbitas escogidas en el plano $\left(r, P_{r}\right)$ se reduce a un punto. Esto implica que $E_{r}=0$ (ver A y por tanto que la orbita solo vibra en los modos $R$ y $\theta$. Se trata tambien de un 2-toro. 
(a)

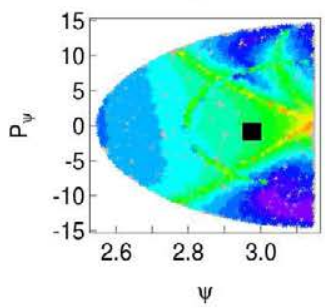

(d)

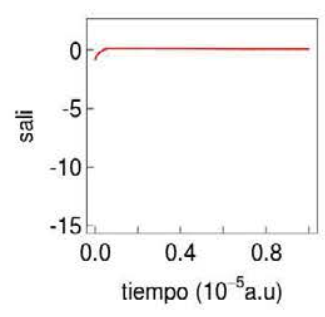

(g)

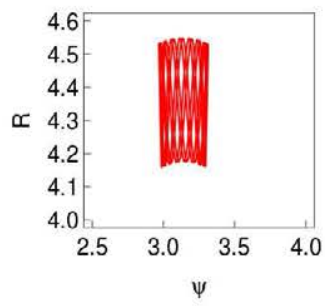

(j)

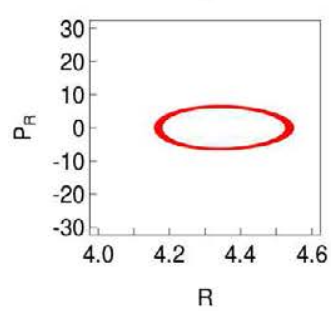

(m)

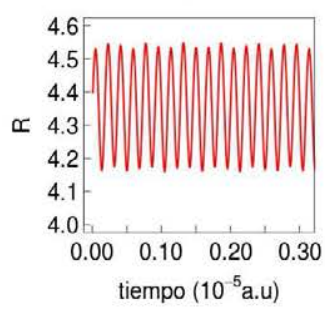

(b)

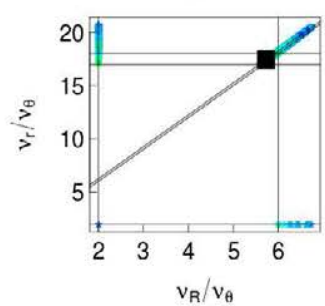

(e)

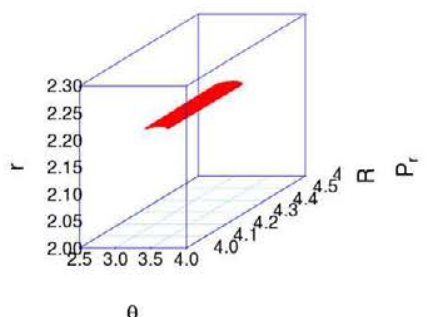

(h)

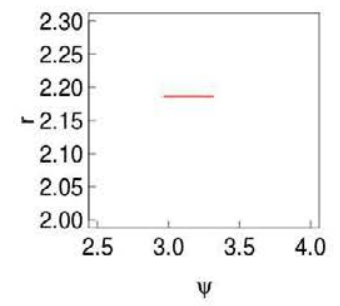

(k)

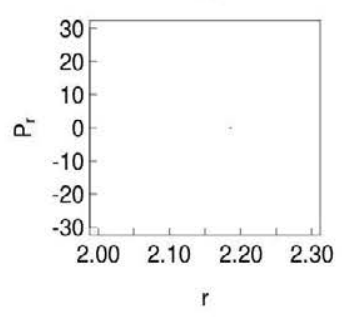

(n)

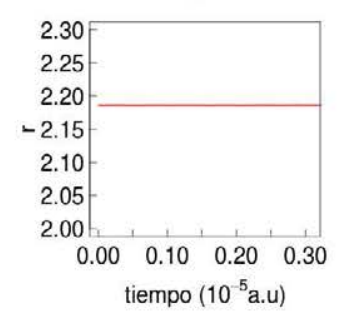

(c)

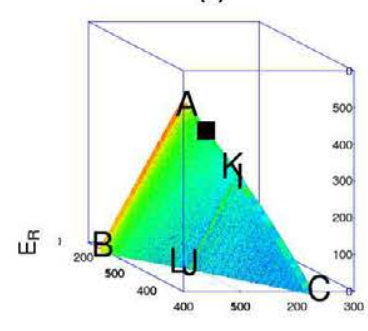

(f)

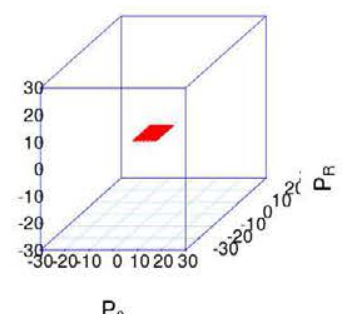

(i)

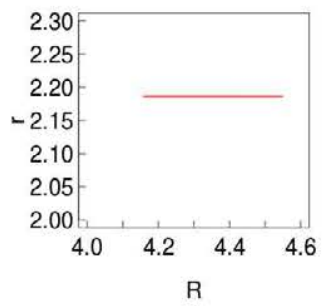

I

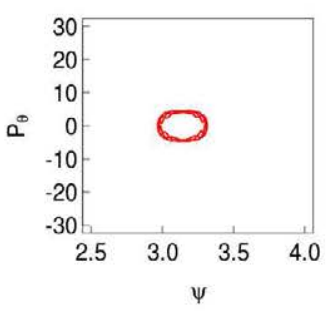

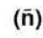

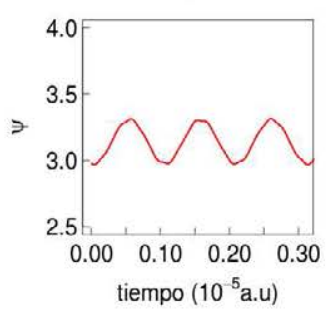

Figura 7.11: Descripción de la órbita b1. 
(a)

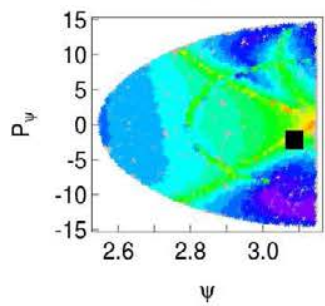

(d)

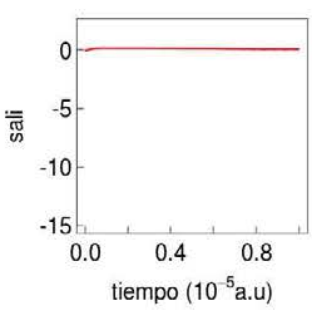

(g)

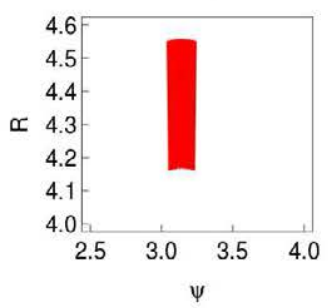

(j)

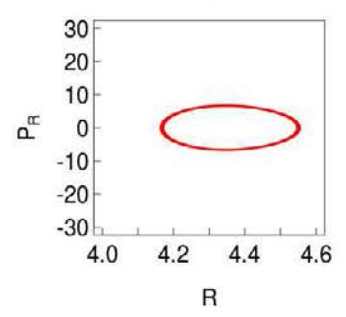

(m)

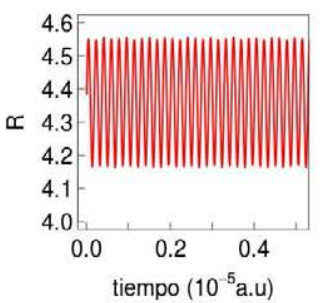

(b)

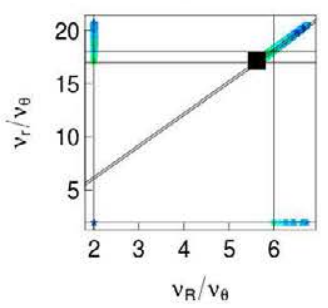

(e)

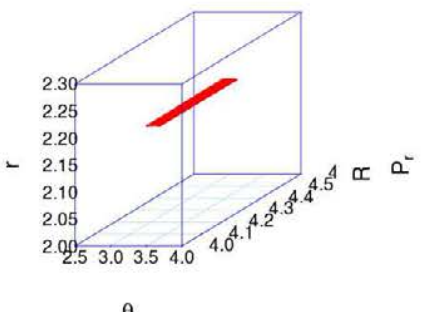

(h)

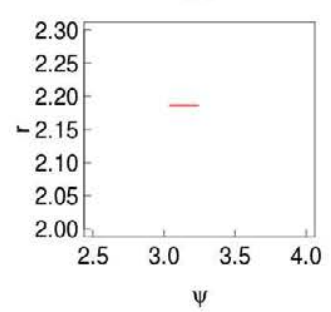

(k)

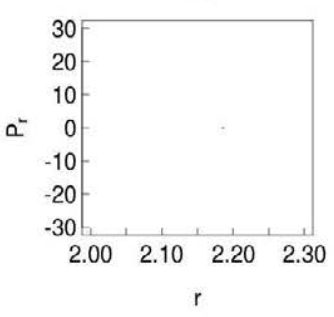

(n)

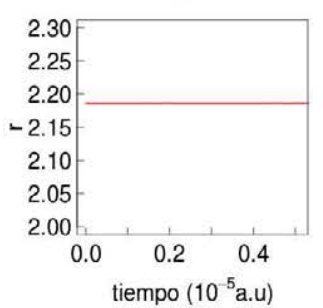

(c)

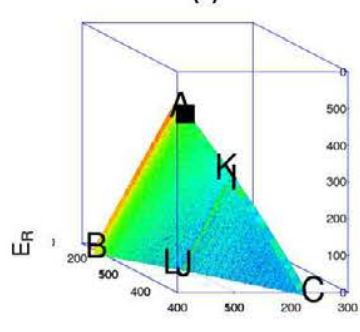

$E_{\theta}$

(f)

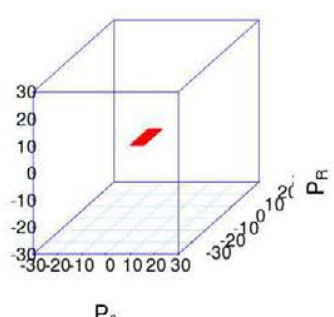

(i)

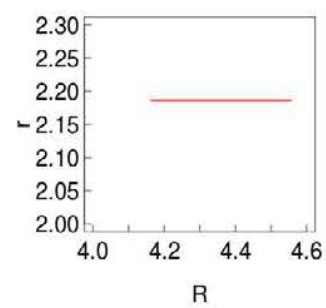

I

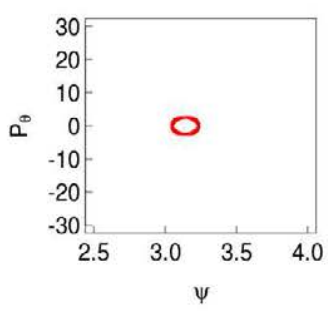

(ท)

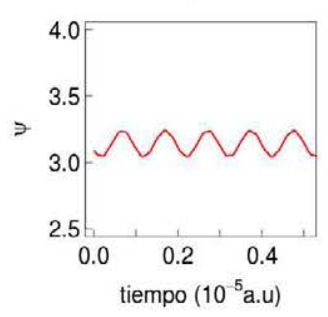

Figura 7.12: Descripción de la órbita b2. 
(a)

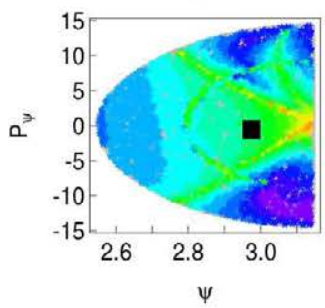

(d)

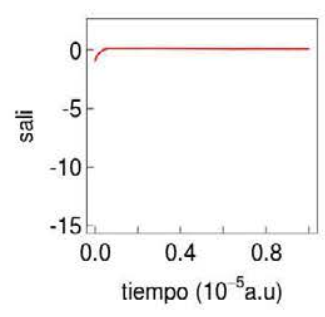

(g)

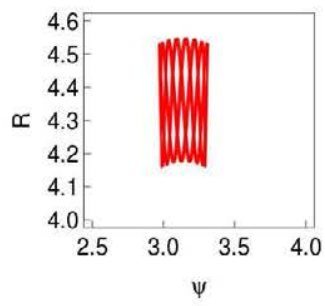

(j)

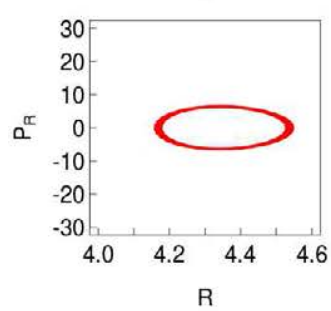

(m)

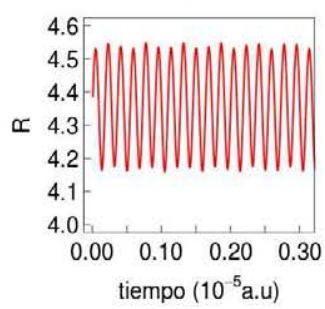

(b)

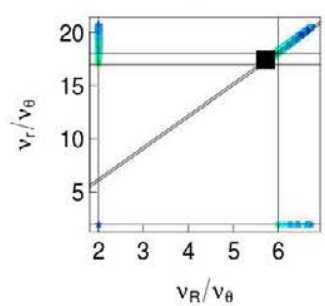

(e)

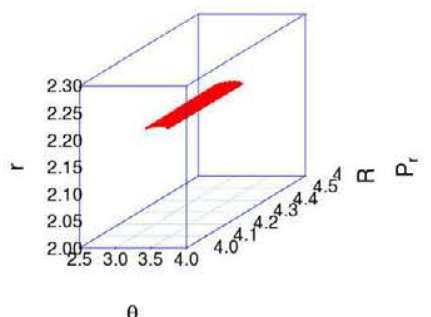

(h)

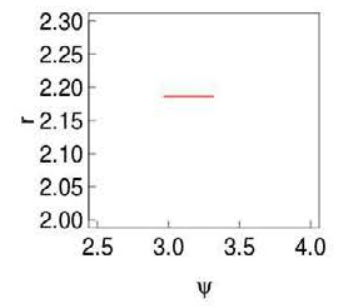

(k)

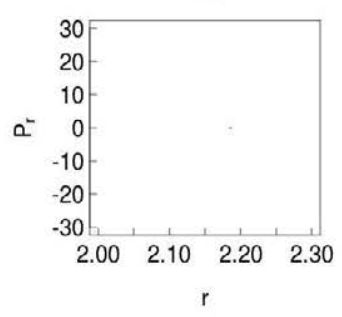

(n)

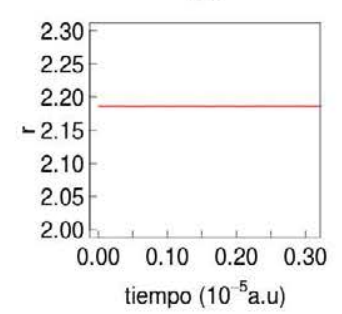

(c)

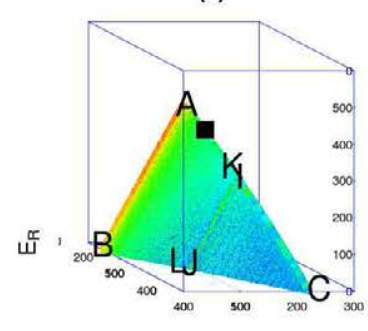

(f)

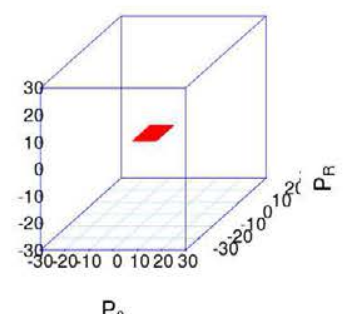

(i)

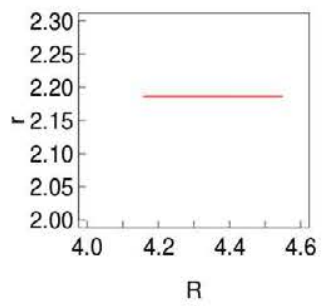

I

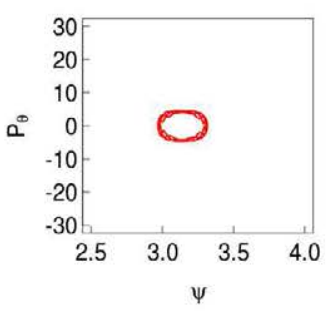

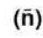

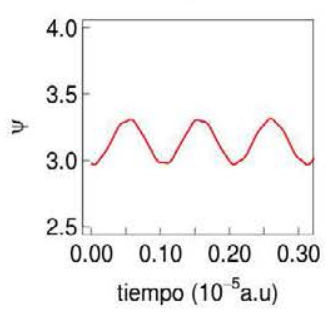

Figura 7.13: Descripsción de la órbita b3. 


\subsection{3. Órbitas en la resonancia $(0,1,-17)$}
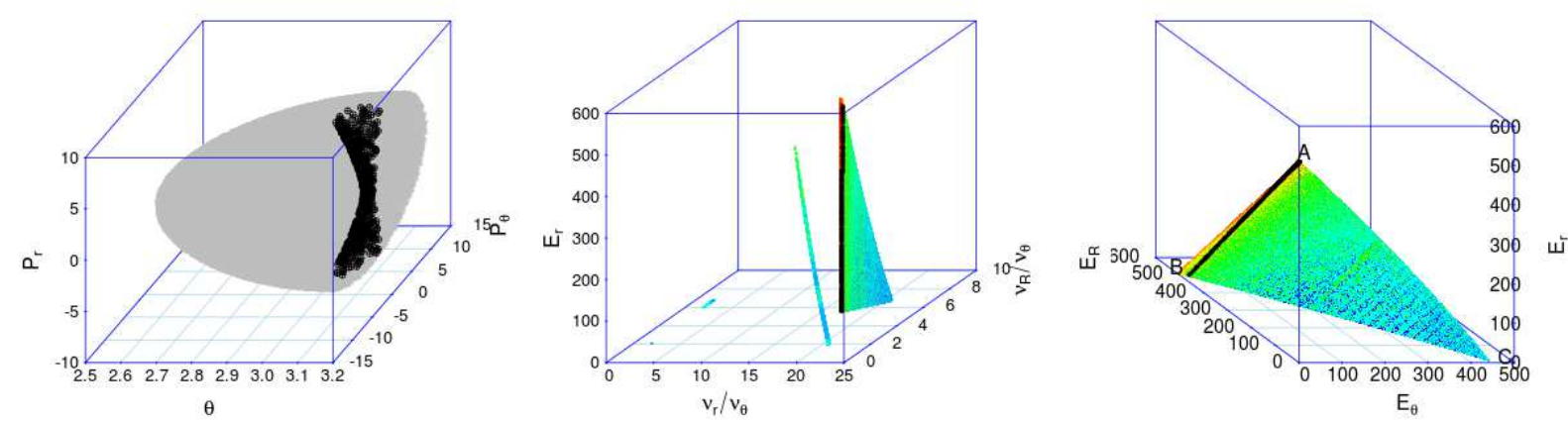

Figura 7.14: Grupo (c). Órbitas ligadas a la resonancia $(0,1,-17)$ entre $r$ y $\theta$.Se muestra la posición de las órbitas con puntos negros en los distintos mapas: $S M_{3 D}$ (izquierda), $M F E_{3 D}$ (centro) y $M E M_{3 D}$ (derecha).

Analizaremos en este grupo las órbitas cuya relación de frecuencias es $(0,1,-17)$. Dichas órbitas se agrupan en el segmento $\overline{A B}$ del mapa $M F E_{3 D}$ y el segmento $\overline{A B}$ del mapa $M E M_{3 D}$. En el mapa $S M_{3 D}$ se observa que las órbitas (representadas como puntos negros en el diagrama 3D del mapa) se agrupan en la syperficie de un hiperboloide de una hoja cuyo eje es la recta perpendicular al plano $\psi, P_{\psi}$ que pasa por el pozo de potencial $\psi=\pi$.

Representamos en las figuras 7.15,7.16 y 7.17 ejemplos concretos de órbitas con esta relación de resonancia.

Se puede comprobar (panel (b)) que la relación de resonancia de estas órbitas es efectivamente $(0,1,-17)$ y que las mismas se encuentran en el segmento $\overline{A B}$ del diagrama correspondiente al mapa $M E M_{3 D}$ (panel (c)).

El indicador $S A L I$ muestra que las orbitas escogidos son estables,panel (d). Esto significa que debe estar inmersas en un toro.

Se observa en el corte con el diagrama del mapa $S M_{3 D}$ (panel (a)) que las órbitas escogidas se encuentran en el interior del corte, cerca del pozo $\psi=\pi$.

Como en el caso anterior, podemos comprobar en el panel(k) que la proyección de las órbitas escogidas en el plano $\left(\psi, P_{\psi}\right)$ se reduce a un punto. Esto implica que $E_{\psi}=0$ (ver A y por tanto que la orbita solo vibra en los modos $R$ y $r$. Se trata por tanto tambien de un 2-toro. 
(a)

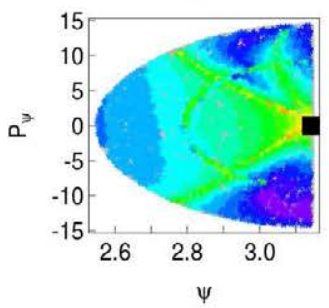

(d)

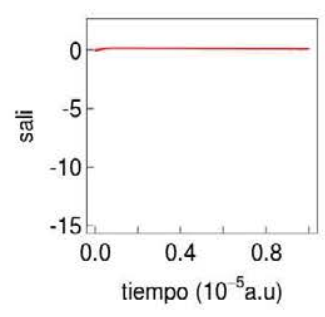

(g)

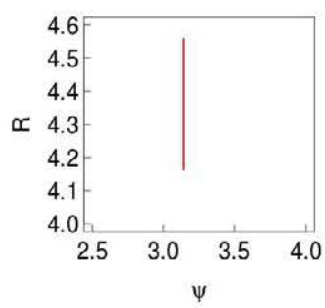

(j)

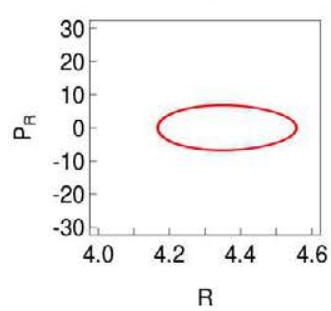

(m)

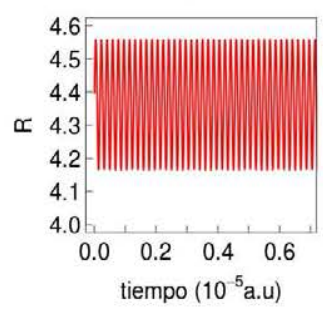

(b)

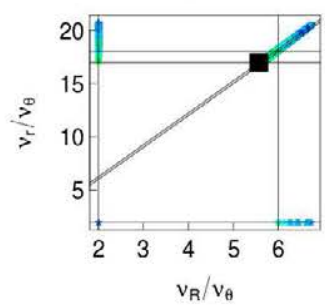

(e)

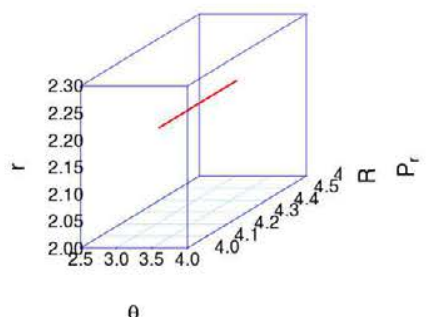

(h)

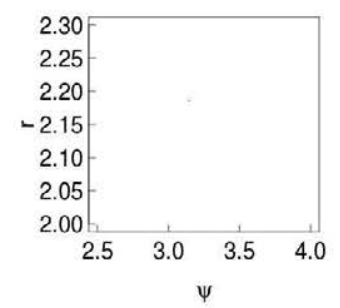

(k)

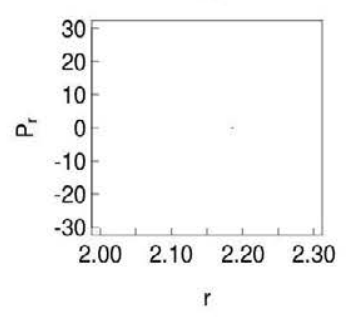

(n)

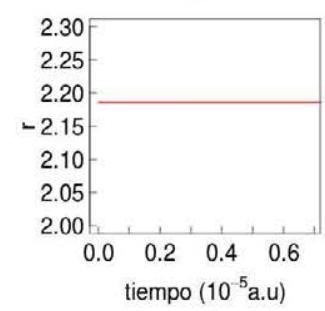

(c)

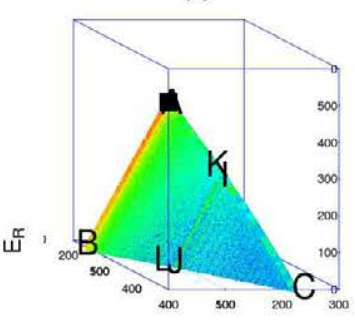

(f)

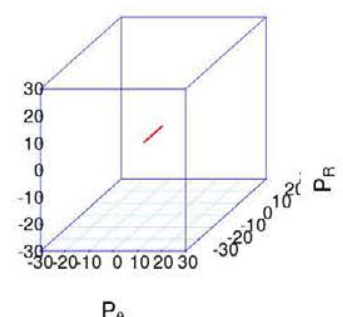

(i)

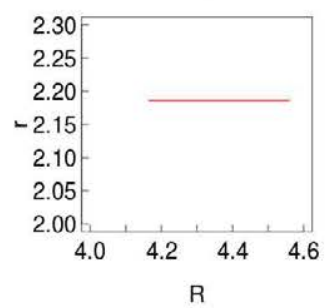

I

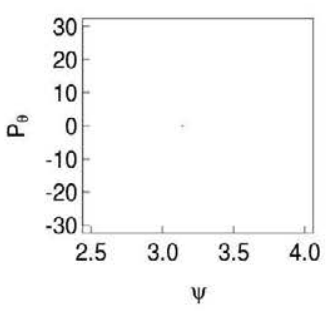

(n)

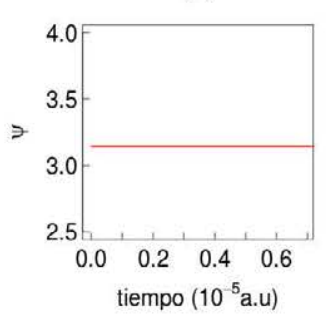

Figura 7.15: Descripción de la órbita c1. 
(a)

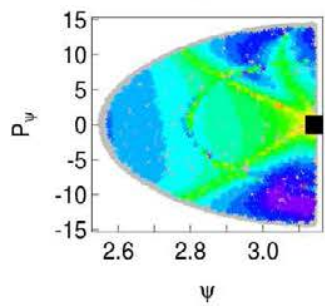

(d)

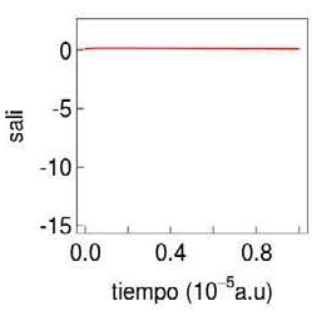

(g)

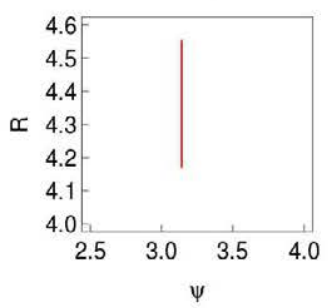

(j)

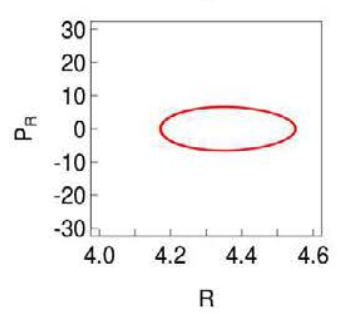

(m)

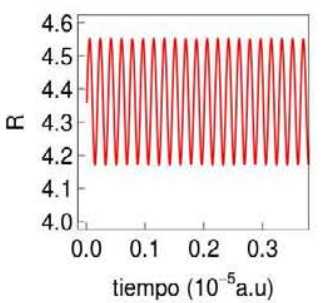

(b)

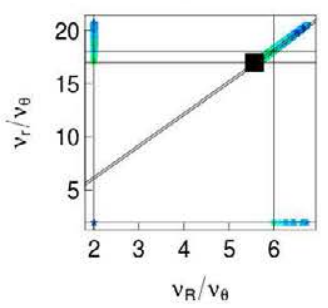

(e)

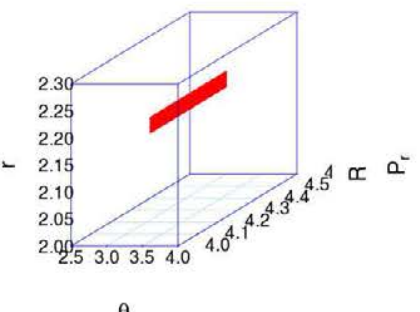

(h)

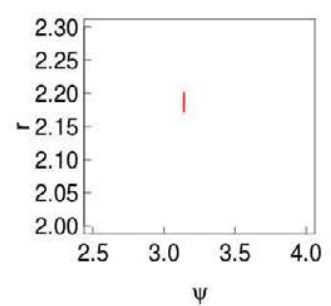

(k)

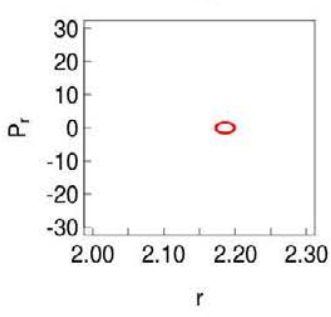

(n)

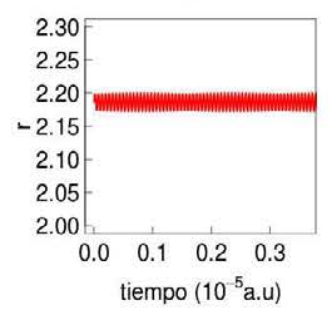

(c)

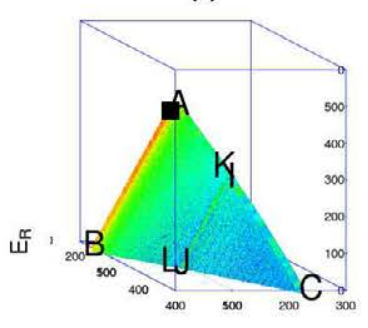

(f)

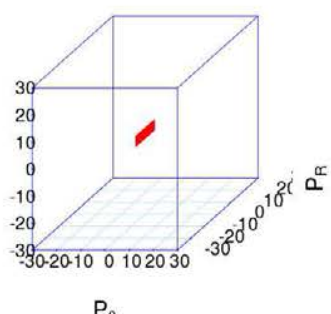

(i)

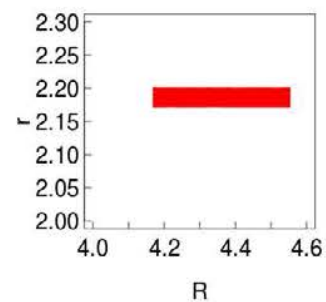

I

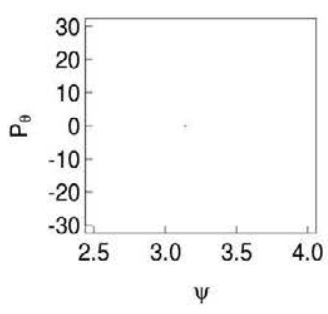

(ท̄)

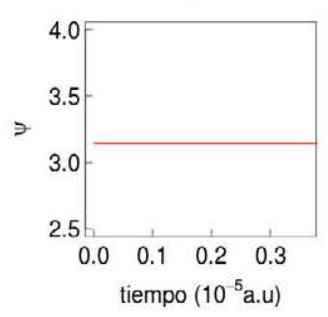

Figura 7.16: Descripción de la órbita c2. 
(a)

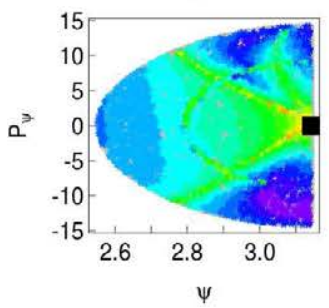

(d)

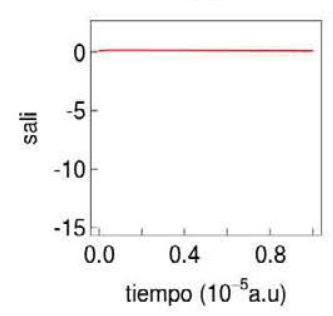

(g)

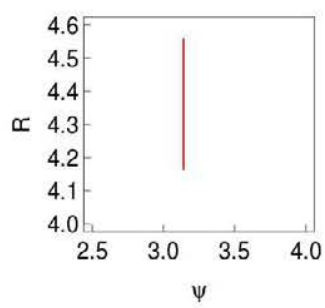

(j)

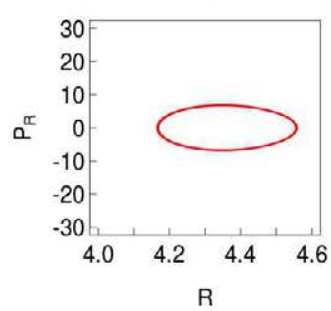

(m)

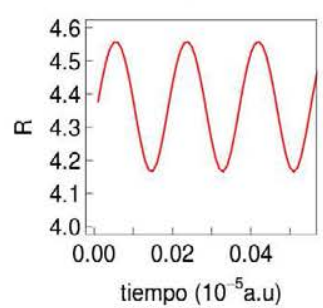

(b)

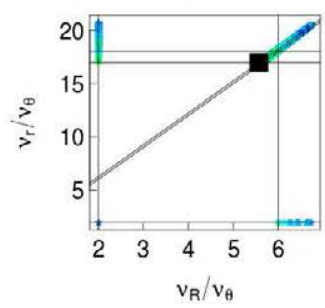

(e)

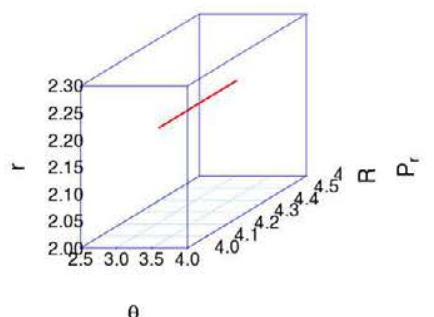

(h)

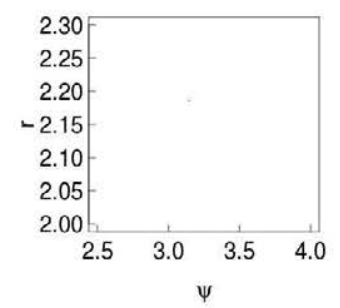

(k)

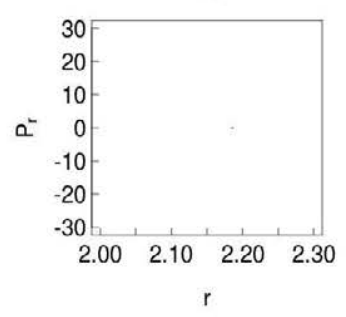

(n)

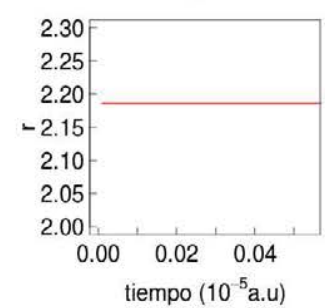

(c)

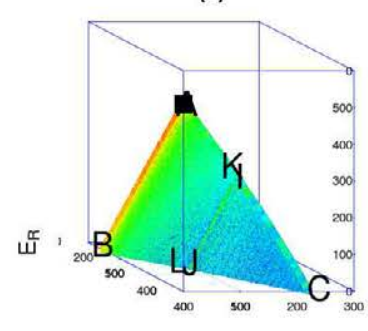

(f)

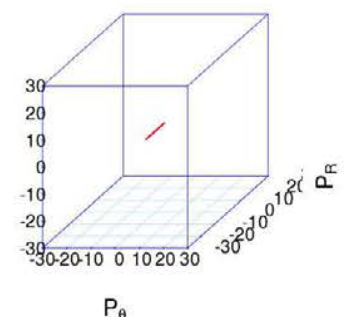

(i)

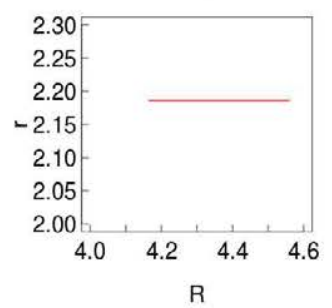

I

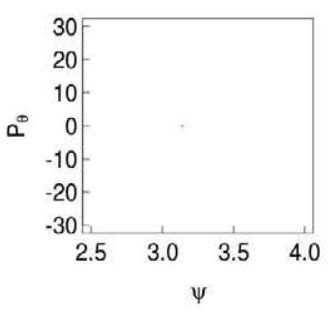

(n)

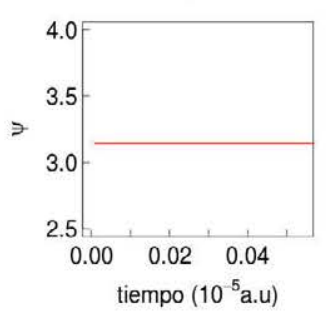

Figura 7.17: Descripción de la órbita c3. 


\subsection{4. Órbitas en la resonancia $(1,0,-2)$}
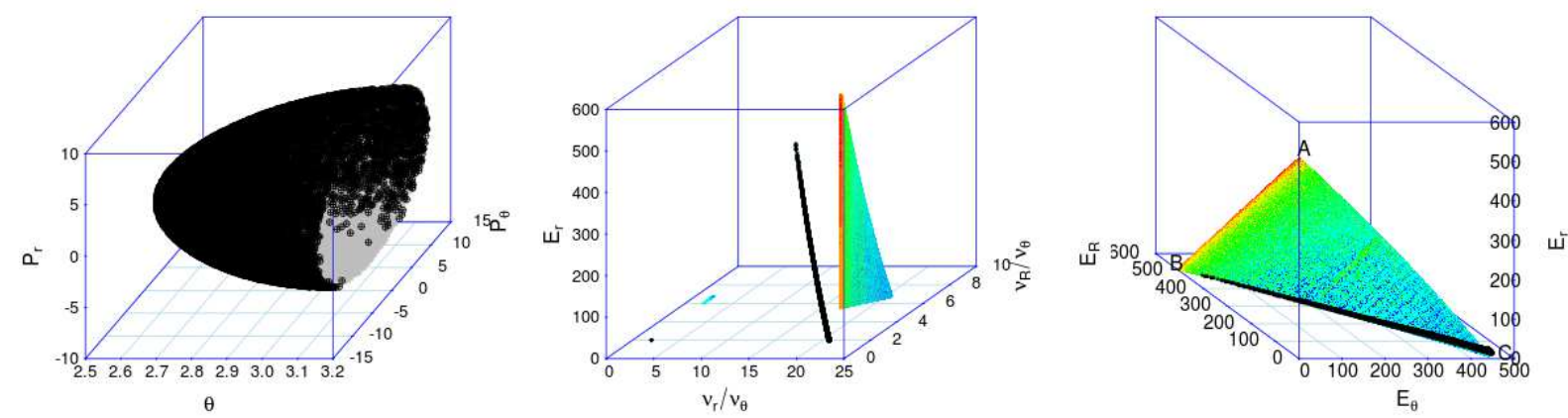

Figura 7.18: Grupo (d). Órbitas ligadas a la resonancia $(1,0,-2)$ entre $R$ y $\theta$.Se muestra la posición de las órbitas con puntos negros en los distintos mapas: $S M_{3 D}$ (izquierda), $M F E_{3 D}$ (centro) y $M E M_{3 D}$ (derecha).

Analizaremos en este grupo las órbitas cuya relación de frecuencias es $(1,0,-2)$. Dichas órbitas se agrupan en el segmento $\overline{D E}$ del mapa $M F E_{3 D}$ y el segmento $\overline{A C}$ del mapa $M E M_{3 D}$. En el mapa $S M_{3 D}$ se observa que las órbitas (representadas como puntos negros en el diagrama 3D del mapa) se agrupan como una nube de puntos en la superficie del elipsoide .

Representamos en las figuras 7.19,7.20 y 7.21 ejemplos concretos de órbitas con esta relación de resonancia.

Se puede comprobar (panel (b)) que la relación de resonancia de estas órbitas es efectivamente $(1,0,-2)$ y que las mismas se encuentran en el segmento $\overline{A C}$ del diagrama correspondiente al mapa $M E M_{3 D}$ (panel (c)).

El indicador $S A L I$ muestra que las orbitas escogidos son estables,panel (d). Esto significa que debe estar inmersas en un toro.

Se observa en el corte con el diagrama del mapa $S M_{3 D}$ (panel (a)) que las órbitas escogidas se encuentran en el interior del corte, en el entorno del pozo $\psi=\pi$.

Podemos comprobar en el panel(l) que la proyección de las órbitas escogidas en el plano $\left(\psi, P_{\psi}\right)$ se reduce a un punto. Esto implica que $E_{\psi}=0$ (ver A y por tanto que la orbita solo vibra en los modos $R$ y $r$. Se trata tambien de un 2-toro. 
(a)

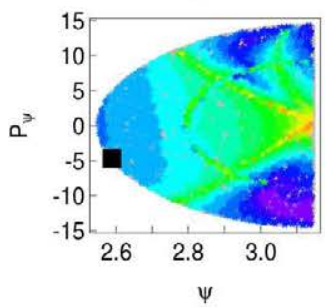

(d)

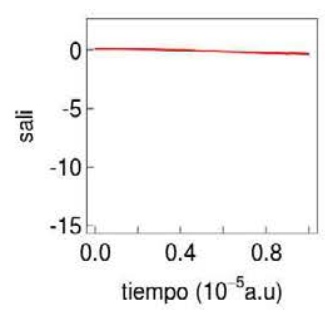

(g)

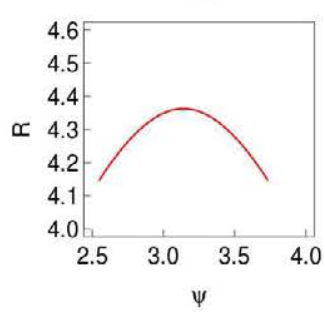

(j)

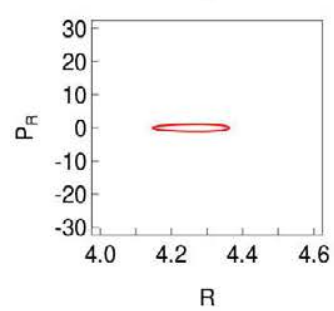

(m)

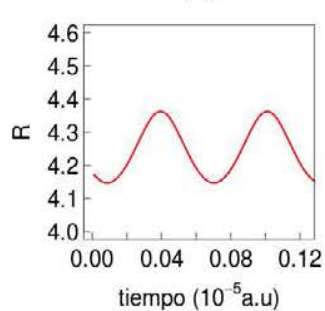

(b)

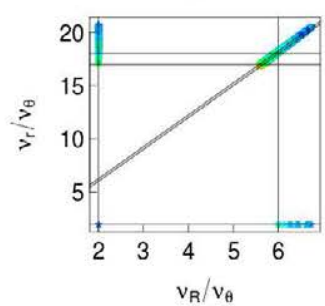

(e)

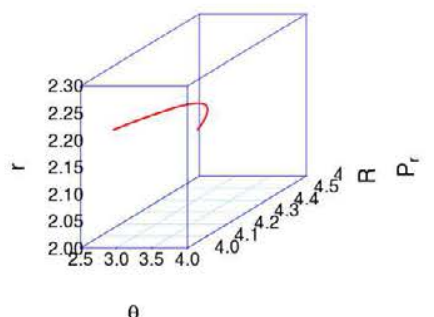

(h)

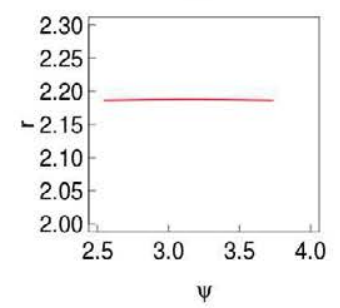

(k)

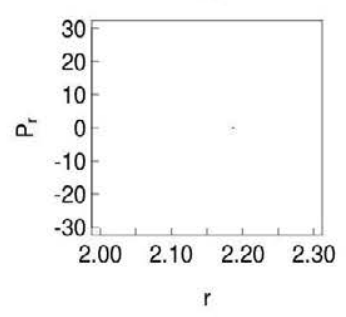

(n)

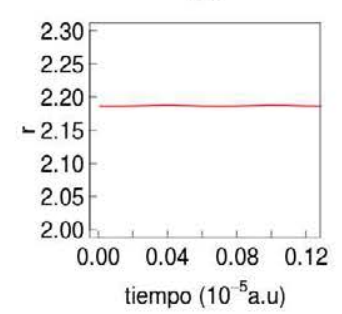

(c)

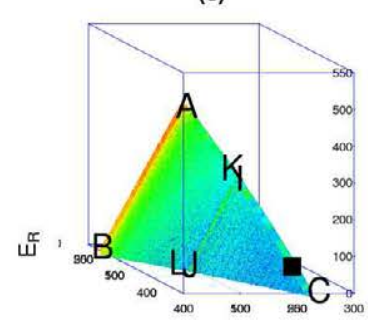

(f)

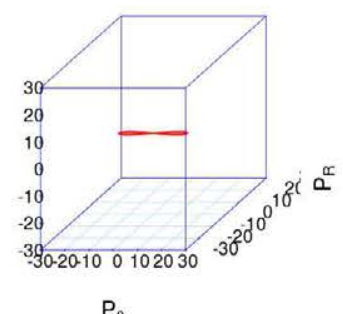

(i)

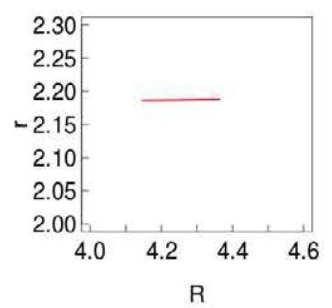

I

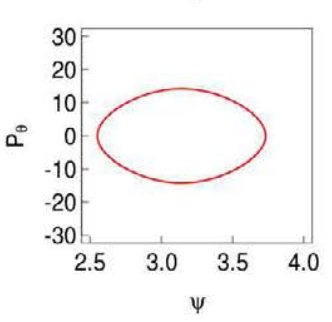

(n)

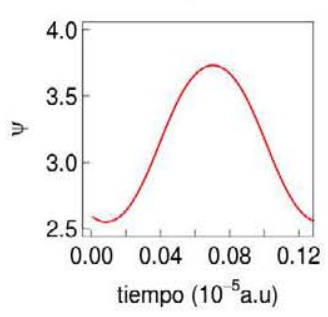

Figura 7.19: Descripción de la órbita d1. 
(a)

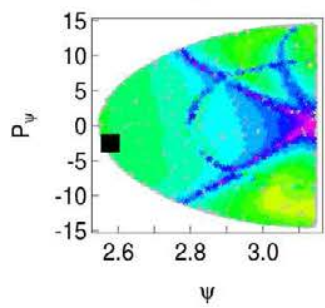

(d)

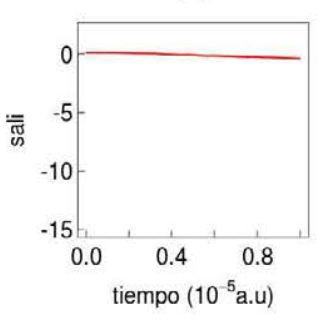

(g)

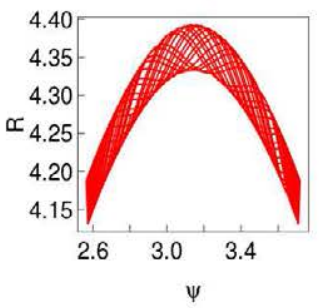

(j)

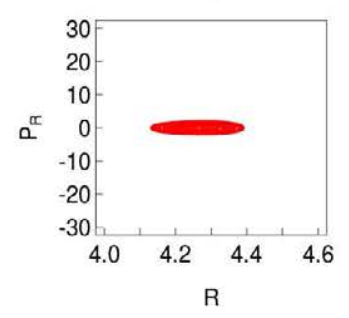

(m)

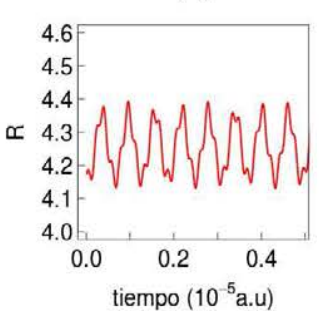

(b)

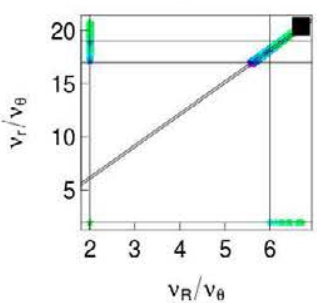

(e)

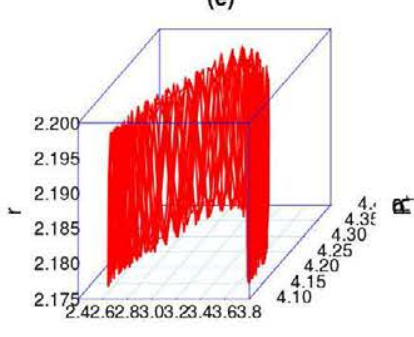

$\theta$

(h)

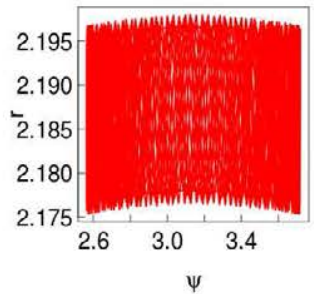

(k)

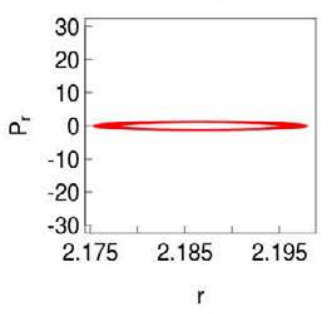

(n)

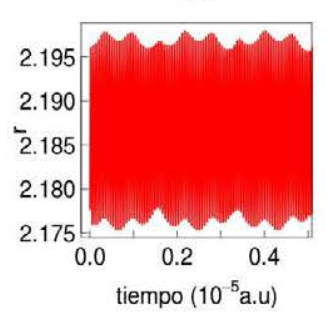

(c)

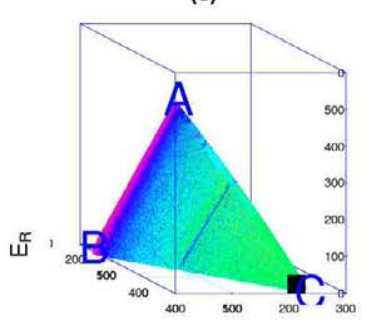

(f) $E_{\theta}$

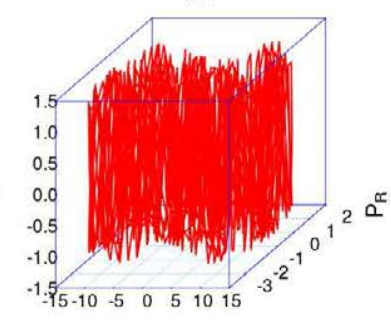

$\mathrm{P}_{\theta}$

(i)
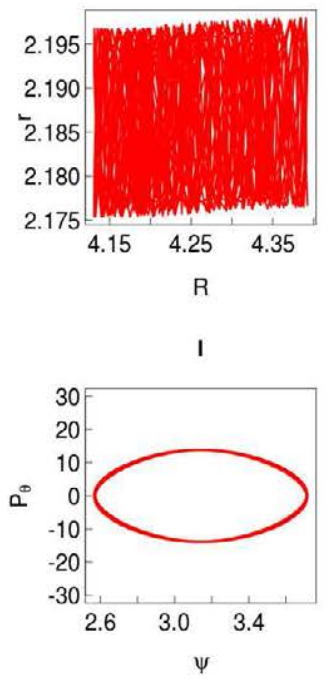

(n)

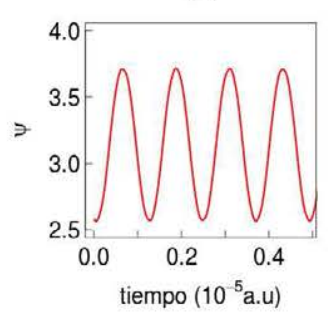

Figura 7.20: Descripción de laórbita d2. 
(a)

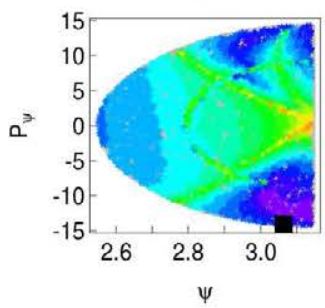

(d)

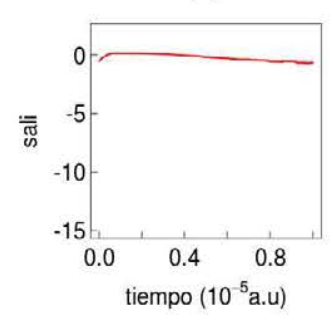

(g)

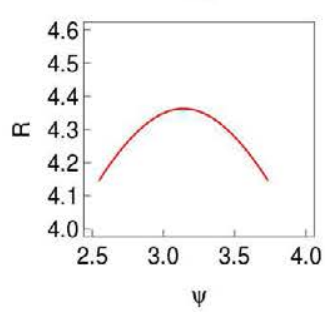

(j)

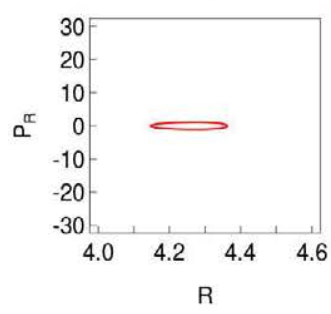

(m)

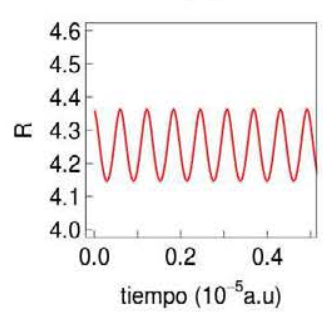

(b)

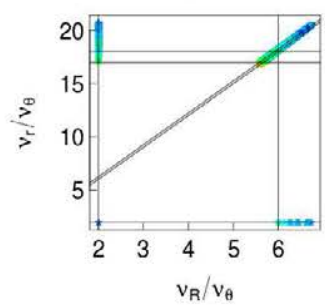

(e)

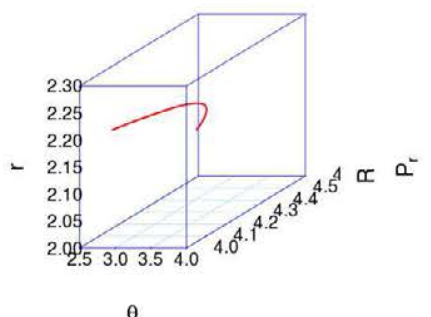

(h)

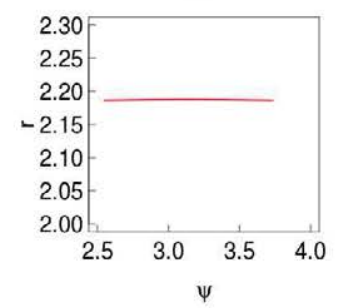

(k)

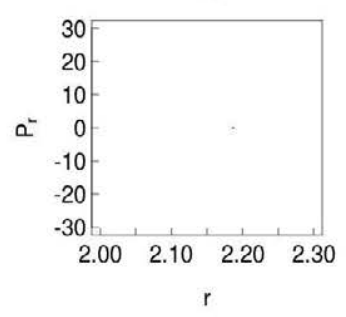

(n)

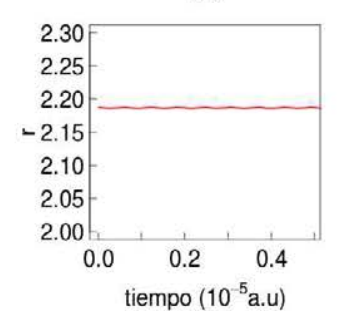

(c)

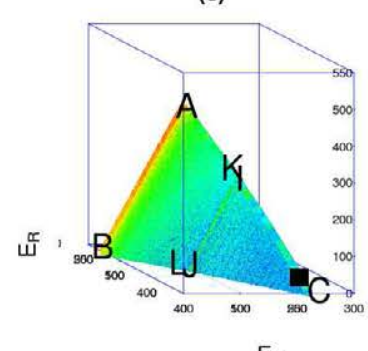

(f)

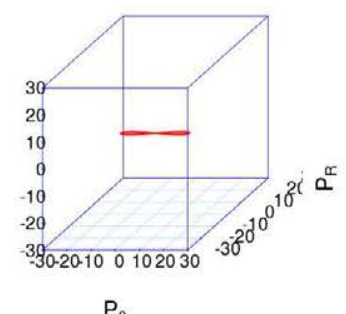

(i)

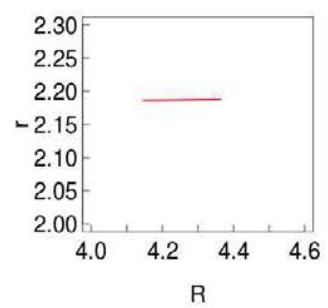

I

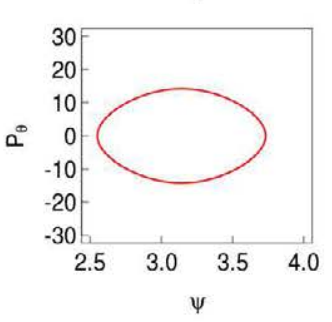

(立)

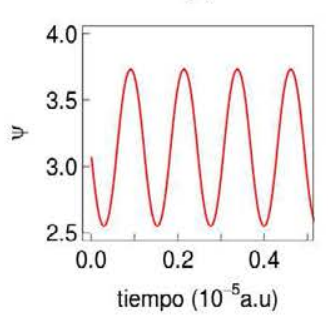

Figura 7.21: Descripción de la órbita d3. 


\subsection{5. Órbitas en la resonancia $(1,0,-6)$}
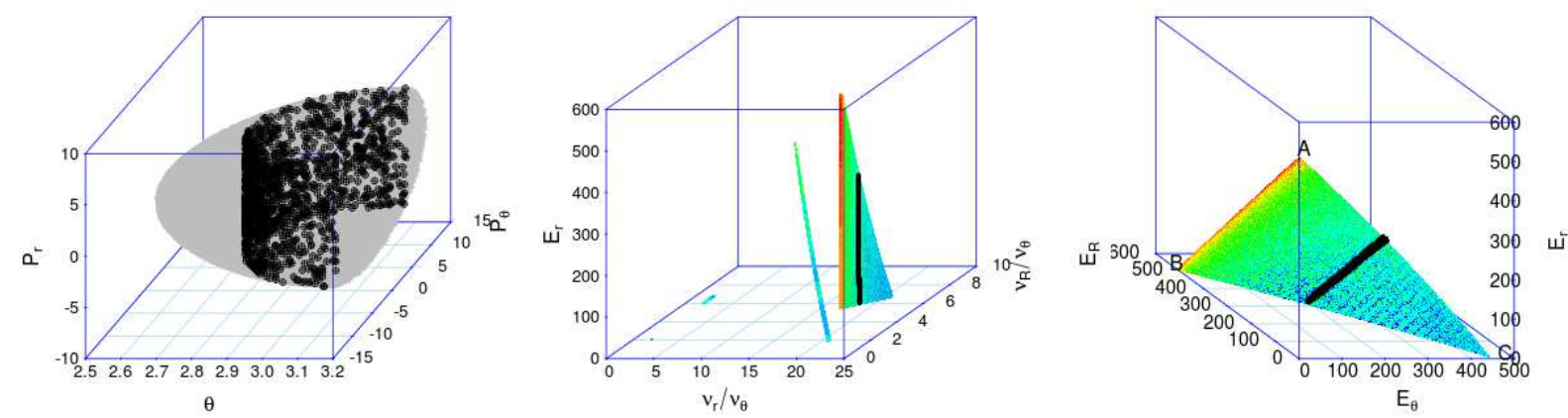

Figura 7.22: Grupo (e). Órbitas ligadas a la resonancia $(1,0,-6)$ entre $R$ y $\theta$.Se muestra la posición de las órbitas con puntos negros en los distintos mapas: $S M_{3 D}$ (izquierda), $M F E_{3 D}$ (centro) y $M E M_{3 D}$ (derecha).

Analizaremos en este grupo las órbitas cuya relación de frecuencias es $(1,0,-6)$. Dichas órbitas se agrupan en el segmento $\overline{I J}$ de los mapas $M F E_{3 D}$ y $M E M_{3 D}$. En el mapa $S M_{3 D}$ se observa que las órbitas (representadas como puntos negros en el diagrama 3D del mapa) se agrupan en la superficie de un paraboloide. Se puede comprobar, figura 7.22 panel derecho, que la resonancia solo existen en el intervalo $[E r I, E r J]$ u.a.

Representamos en las figuras 7.23,7.24 y 7.25 ejemplos concretos de órbitas con esta relación de resnancia.

Se puede comprobar (panel (b)) que la relación de resonancia de estas órbitas es efectivamente $(1,0,-6)$ y que las mismas se encuentran en el segmento $\overline{I J}$ del diagrama correspondiente al mapa $M E M_{3 D}$ (panel (c)).

El indicador $S A L I$ muestra que las orbitas escogidos son bastante estables,panel (d). Se puede comprobar, panel (g), que la proyección de las órbitas seleccionadas en el plano $(R, \psi)$ es muy parecida a la que se oberva en el modelo 2D.

Se observa en el corte con el diagrama del mapa $S M_{3 D}$ (panel (a)) que las órbitas escogidas se encuentran en el entorno de la cadena de islas caracterizada por esta relación de resonancia. Podemos comprobar en los paneles $\left((\mathrm{j}),(\mathrm{k})\right.$ y (l) que la proyección de la órbita en los planos $\left(R, P_{R}\right),\left(r, P_{r}\right)$ y $\left(\psi, P_{p} s i\right)$ no se reduce a un punto lo que nos indica que las órbitas de este grupo tienen energía cinética en todos los modos de vibración.. 
(a)

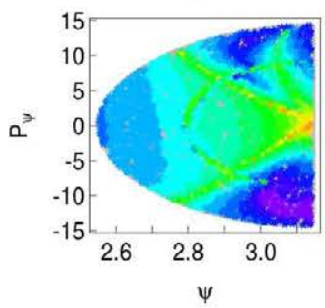

(d)

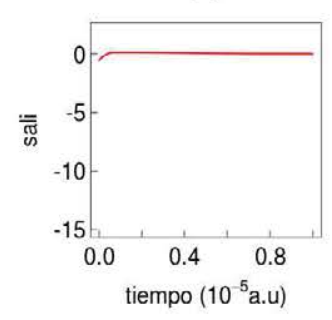

(g)

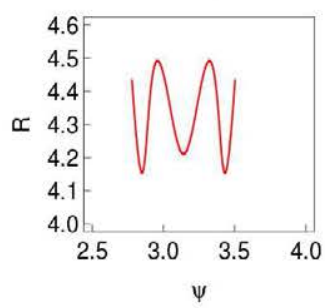

(j)

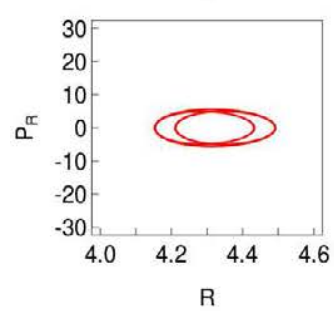

(m)

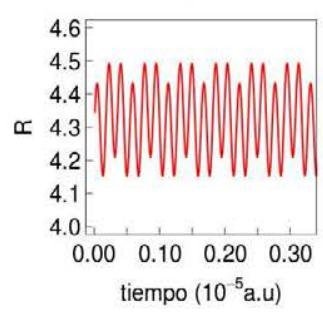

(b)

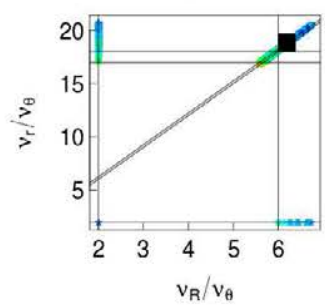

(e)

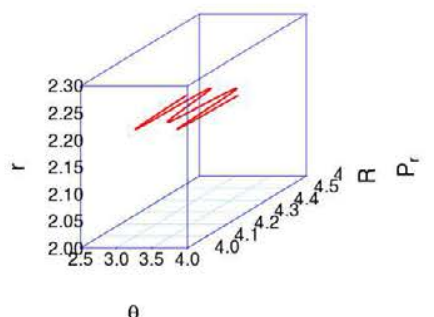

(h)

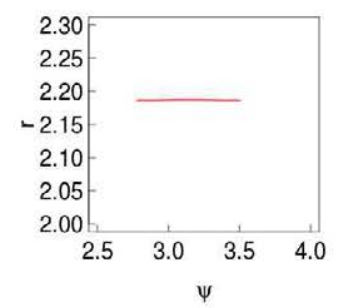

(k)

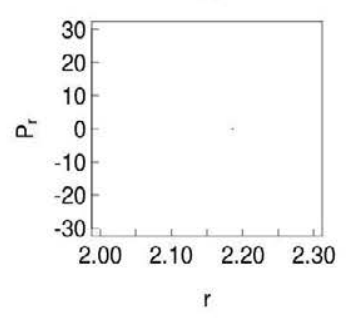

(n)

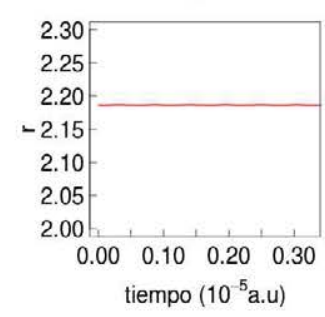

(c)

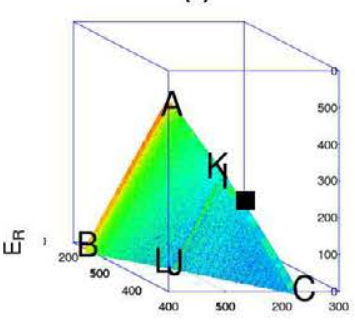

(f)

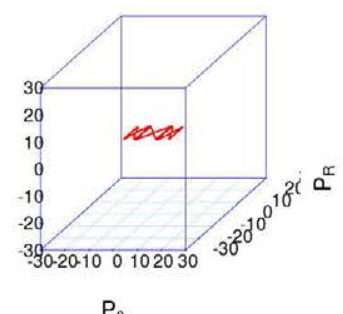

(i)

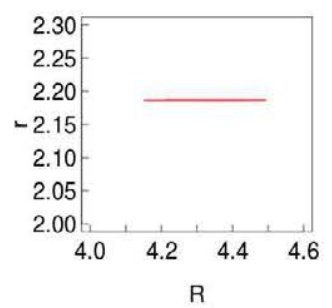

I

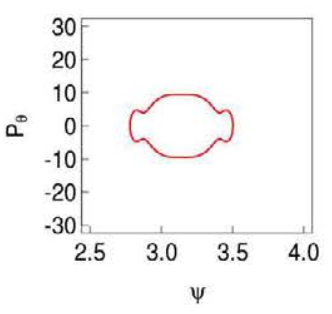

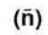

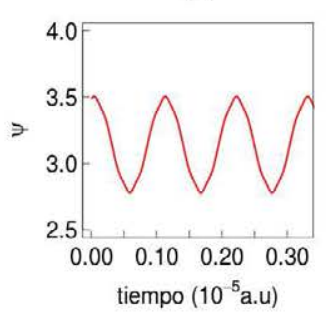

Figura 7.23: Descripción de la órbita e1. 
(a)

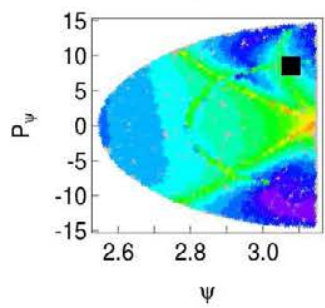

(d)

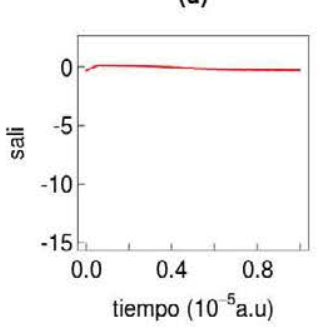

(g)

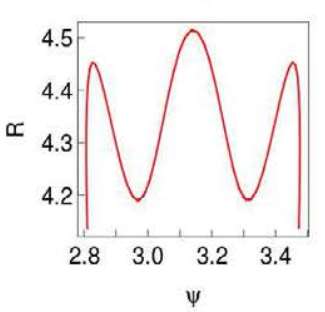

(j)

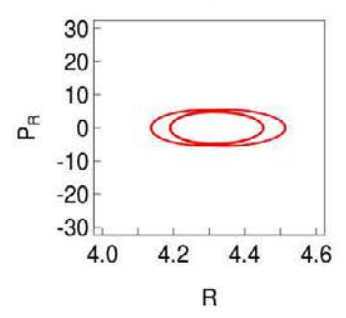

(m)

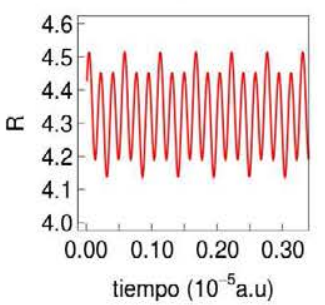

(b)

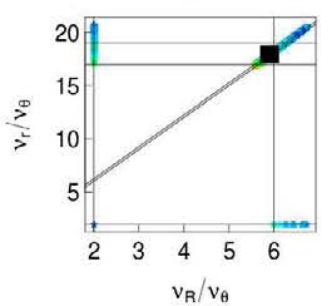

(e)

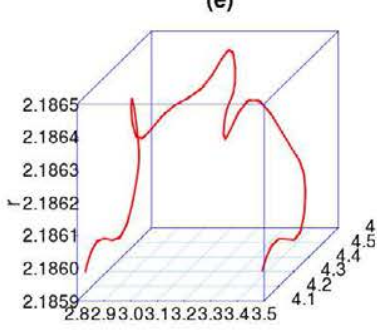

$\theta$

(h)

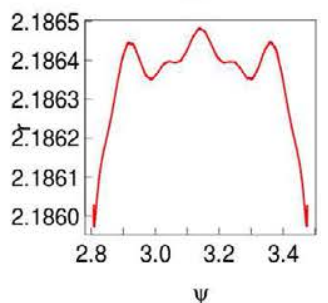

(k)

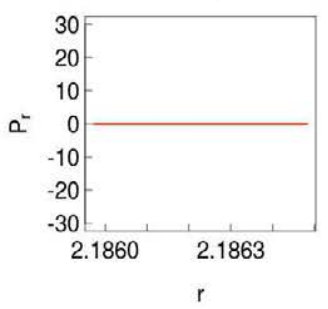

(n)

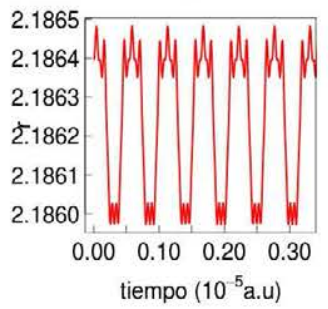

(c)

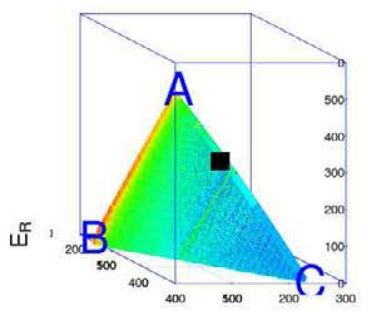

(f) $E_{\theta}$

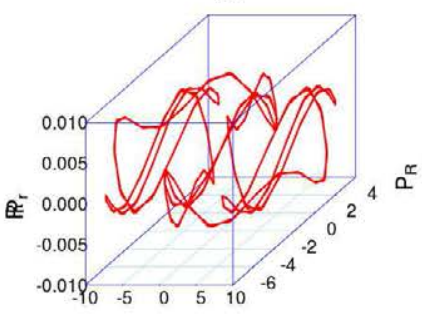

$P_{\theta}$

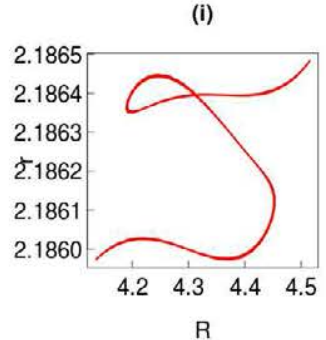

I

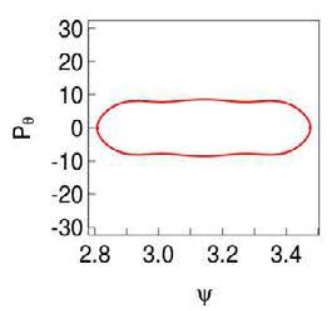

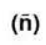

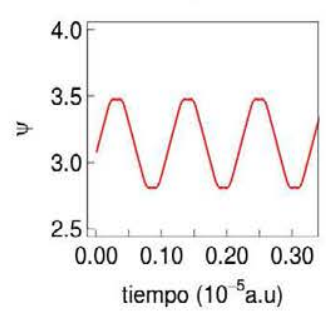

Figura 7.24: Descripción de la órbita e2. 
(a)

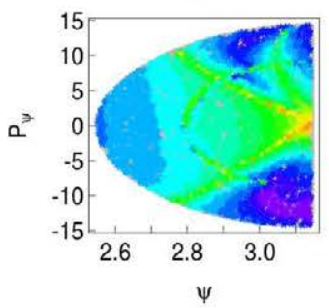

(d)

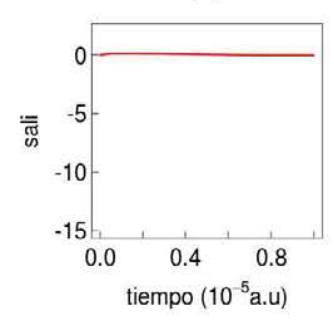

(g)

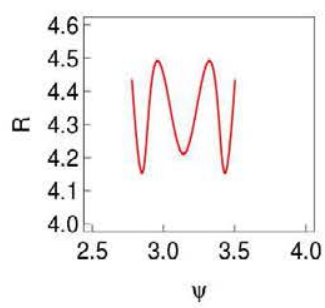

(j)

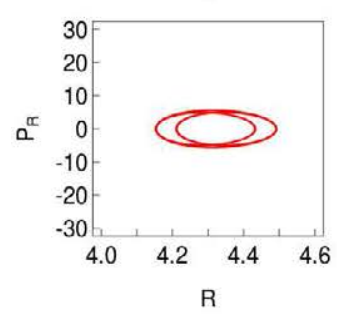

(m)

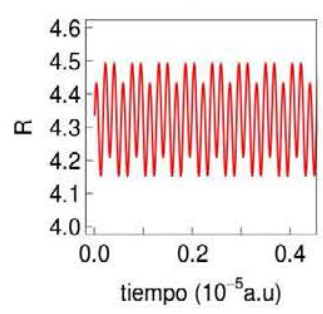

(b)

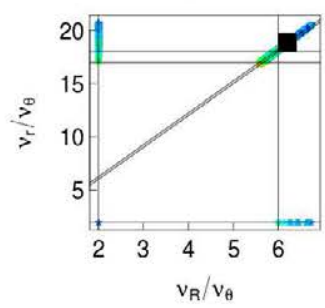

(e)

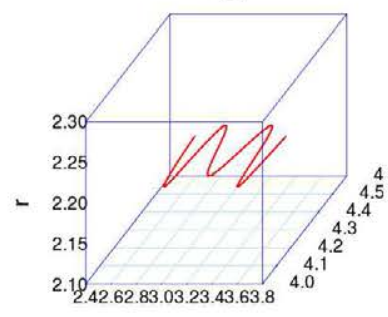

$\theta$

(h)

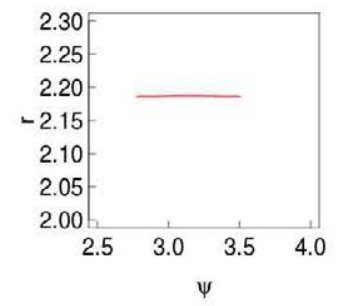

(k)

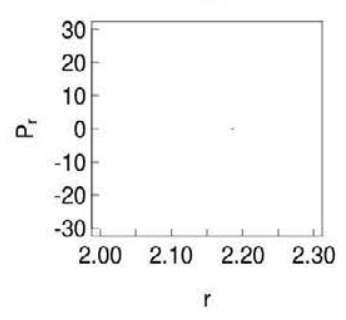

(n)

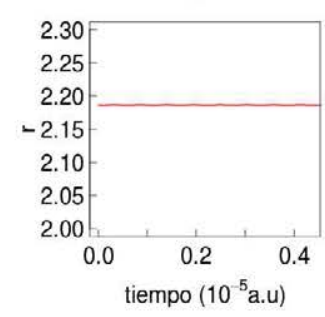

(c)

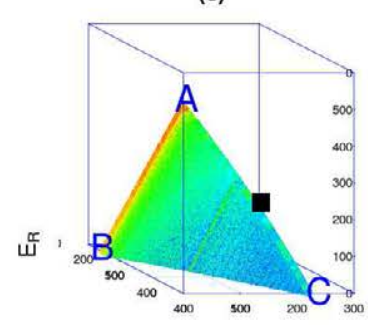

(f) $E_{\theta}$

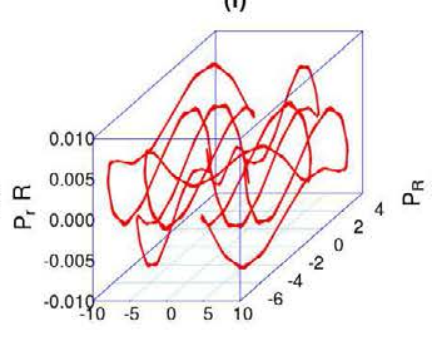

$\mathrm{P}_{\theta}$

(i)

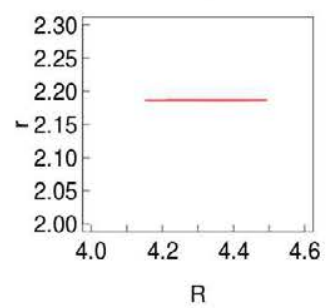

I

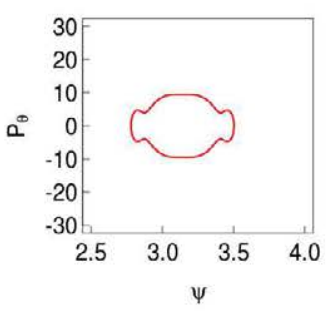

(n)

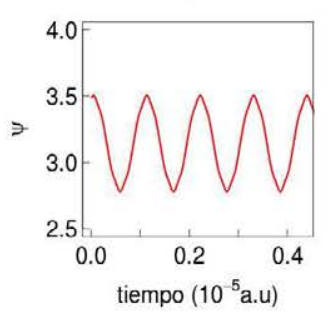

Figura 7.25: Descripción de la órbita e3. 


\subsection{6. Órbitas en la resonancia $(0,1,-18)$}
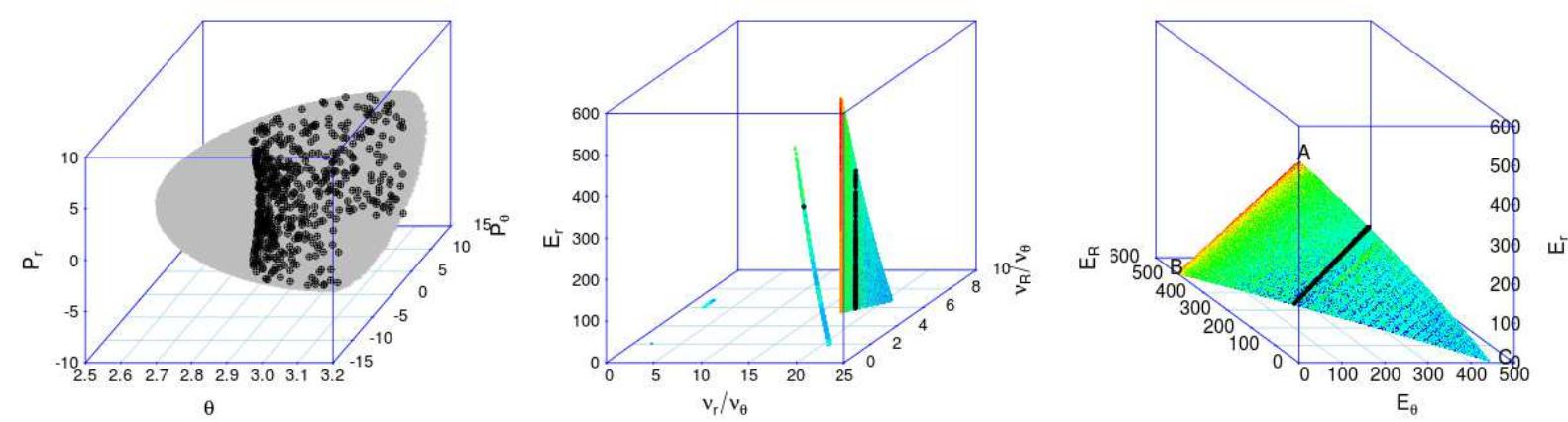

Figura 7.26: Grupo (f). Órbitas ligadas a la resonancia $(0,1,-18)$ entre $r$ y $\theta$.Se muestra la posición de las órbitas con puntos negros en los distintos mapas: $S M_{3 D}$ (izquierda), $M F E_{3 D}$ (centro) y $M E M_{3 D}$ (derecha).

Analizaremos en este grupo las órbitas cuya relación de frecuencias es $(0,1,-18)$. Dichas órbitas se agrupan en el segmento $\overline{K L}$ de los mapas $M F E_{3 D}$ y $M E M_{3 D}$. En el mapa $S M_{3 D}$ se observa que las órbitas (representadas como puntos negros en el diagrama 3D del mapa) se agrupan en la superficie de un paraboloide. Se puede comprobar (7.26 panel derecho) que la resonancia solo existen en el intervalo $[\operatorname{ErI}, \operatorname{Er} J]$ u.a.

Representamos en las figuras 7.27,7.28 y 7.29 ejemplos concretos de órbitas con esta relación de resnancia.

Se puede comprobar (panel (b)) que la relación de resonancia de estas órbitas es efectivamente $(0,1,-18)$ y que las mismas se encuentran en el segmento $\overline{K L}$ del diagrama correspondiente al mapa $M E M_{3 D}$ (panel (c)).

El indicador $S A L I$ muestra que las orbitas escogidos son bastante estables,panel (d). Se puede comprobar, panel (h), que la proyección de las órbitas seleccionadas en el plano $(r, \psi)$ es parecida a la que se oberva en el grupo anterior en el plano $(R, \psi)$.

Se observa en el corte con el diagrama del mapa $S M_{3 D}$ (panel (a)) que las órbitas escogidas se encuentran en el entorno de la cadena de islas caracterizada por esta relación de resonancia. Podemos comprobar en los paneles $\left((\mathrm{j}),(\mathrm{k})\right.$ y $(\mathrm{l})$ que la proyección de la órbita en los planos $\left(R, P_{R}\right),\left(r, P_{r}\right)$ y $\left(\psi, P_{p} s i\right)$ no se reduce a un punto lo que nos indica que las órbitas de este grupo tienen energía cinética en todos los modos de vibración.. 
(a)

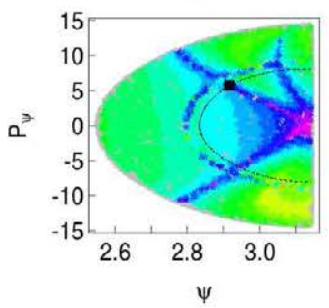

(d)

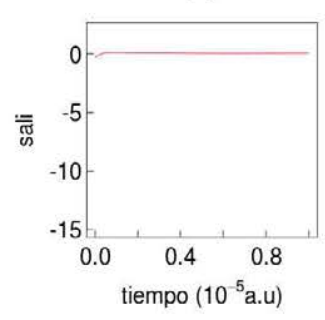

(h)

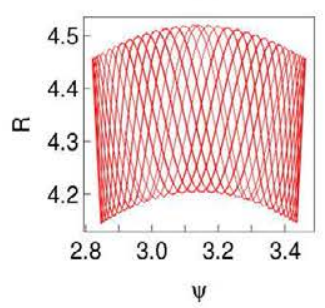

(I)

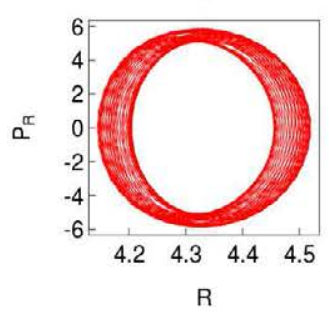

(g)

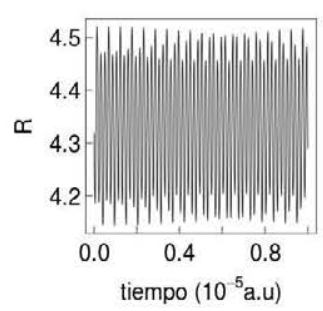

(b)

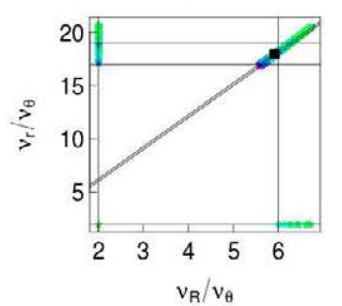

(e)

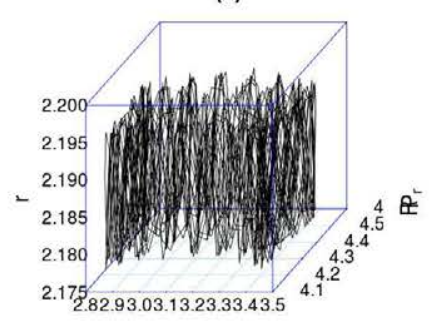

$\theta$

(i)

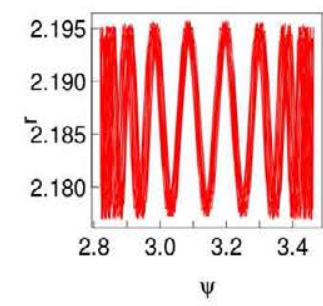

(m)

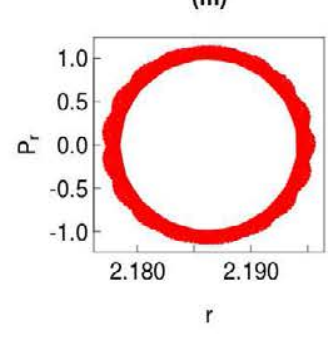

(k)

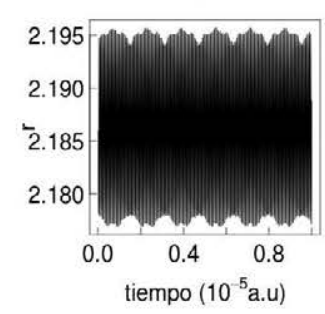

(c)

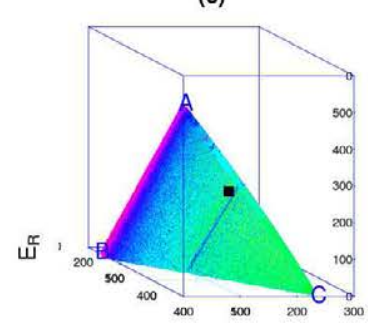

(f) $E_{\theta}$

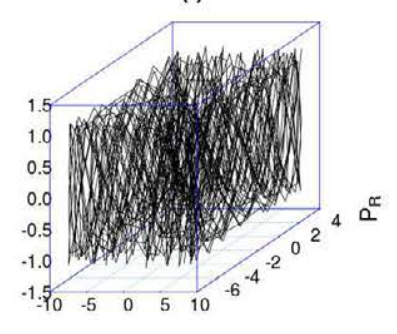

$\mathrm{P}_{\theta}$

(i)

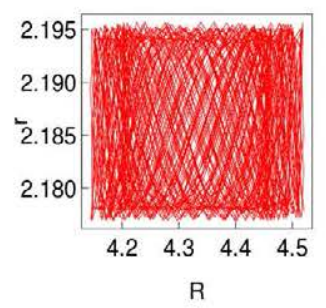

n

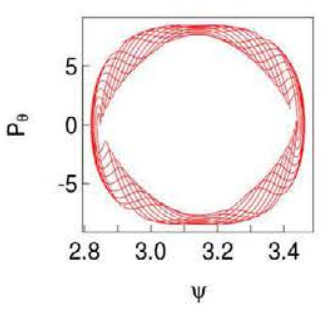

$\overline{\mathbf{n}}$

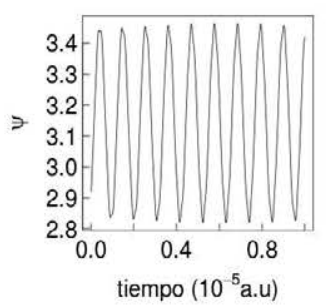

Figura 7.27: Descripción de la órbita f1. 
(a)

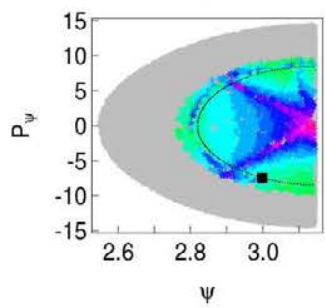

(d)

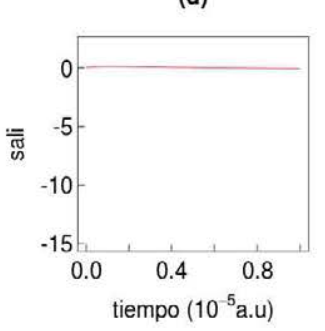

(h)

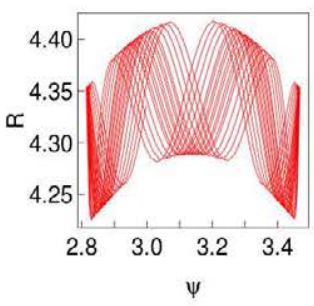

(I)

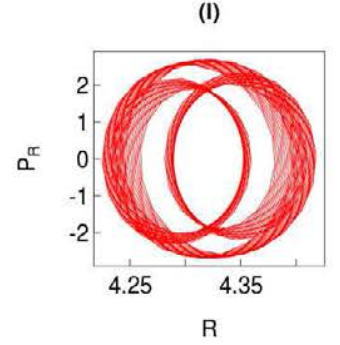

(g)

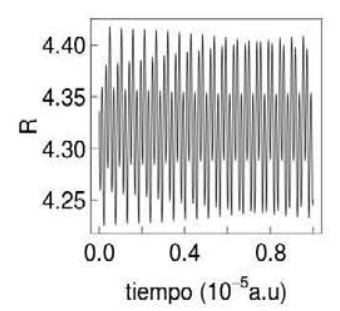

(b)

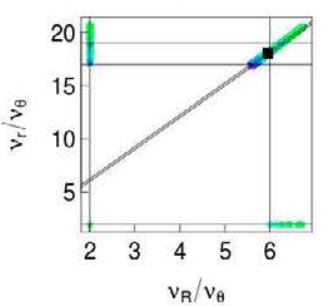

(e)

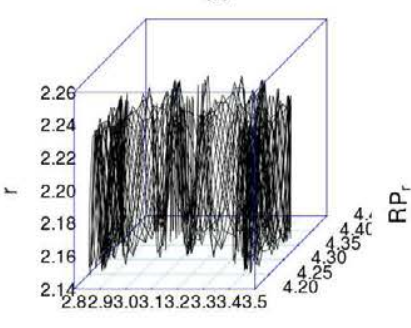

$\theta$

(i)

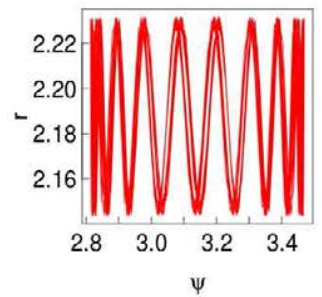

(m)

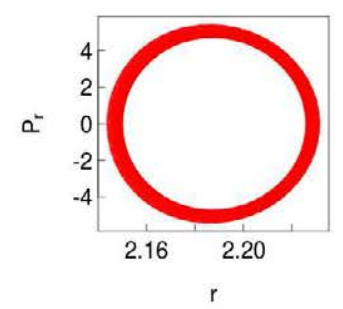

(k)

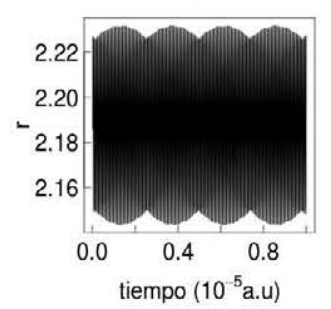

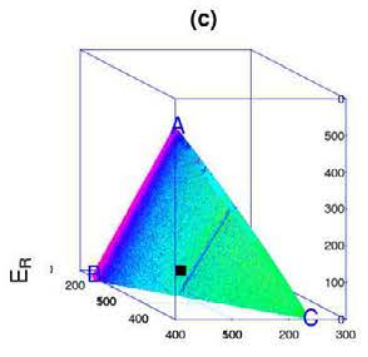

(f) $E_{\theta}$

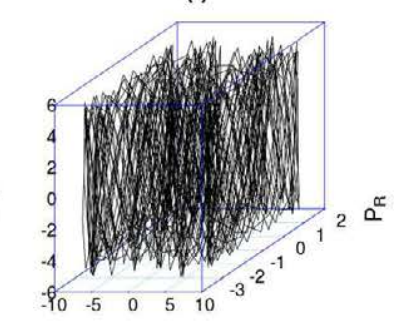

$\mathrm{P}_{\theta}$

(j)

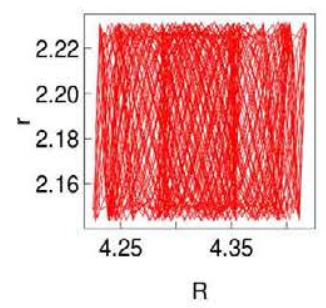

n

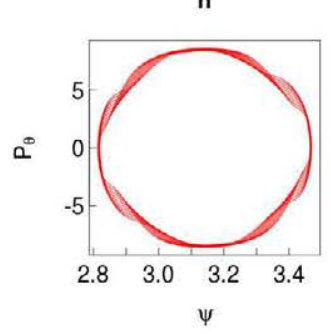

$\overline{\mathbf{n}}$

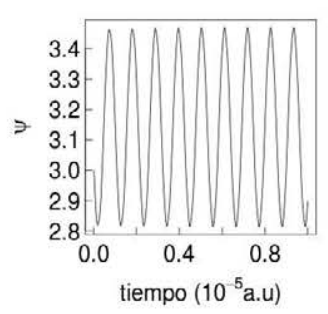

Figura 7.28: Descripción de la órbita f2. 
(a)

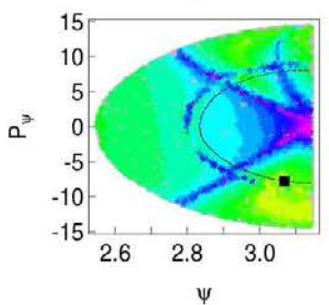

(d)

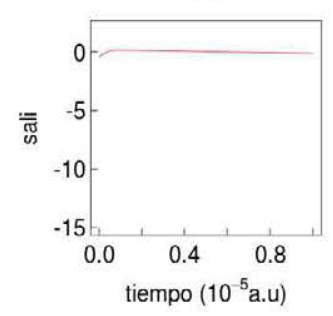

(h)

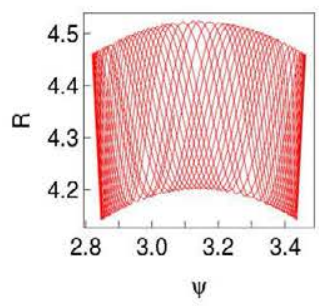

(I)

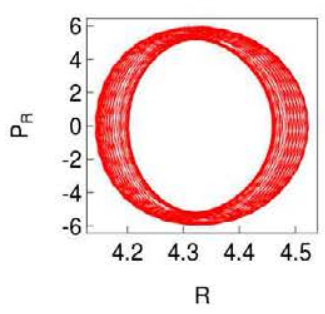

(g)

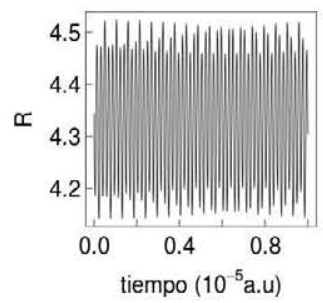

(b)

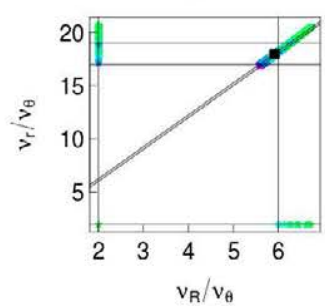

(e)

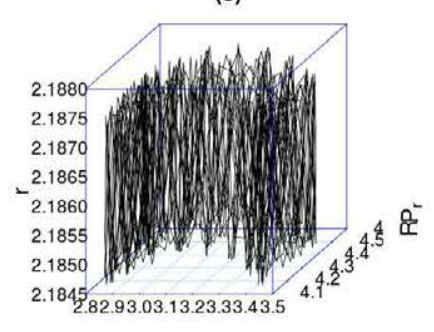

$\theta$

(i)

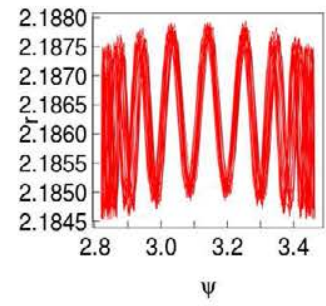

(m)

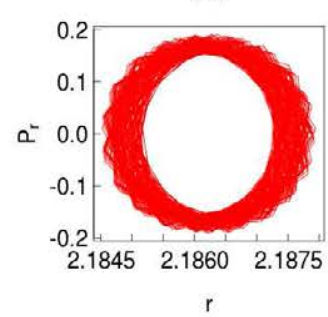

(k)

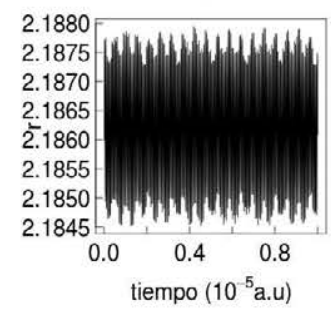

(c)

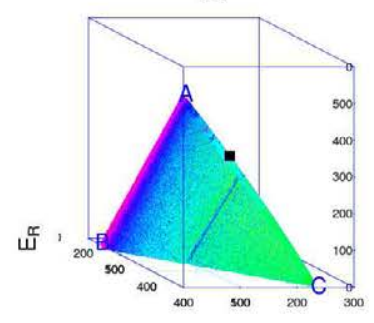

(f) $E_{\theta}$

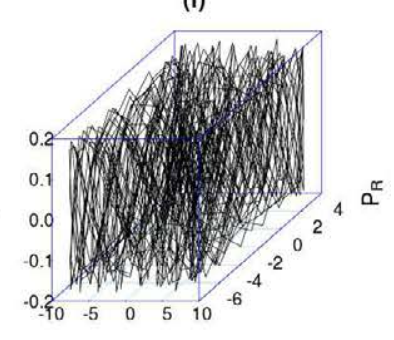

$\mathrm{P}_{\theta}$

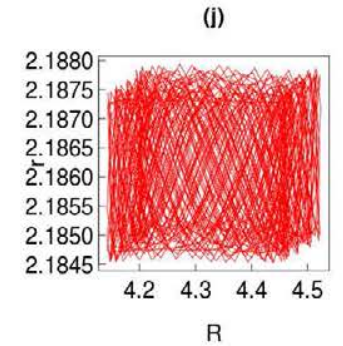

n

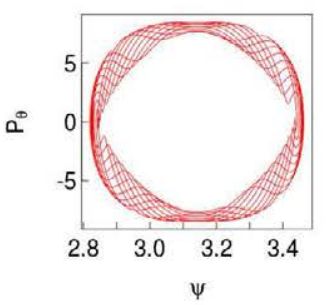

$\overline{\mathbf{n}}$

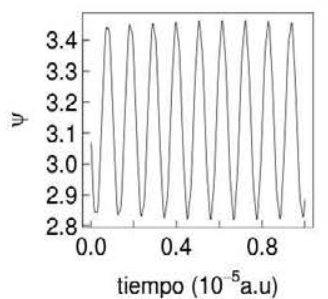

Figura 7.29: Descripción de la órbita f3. 

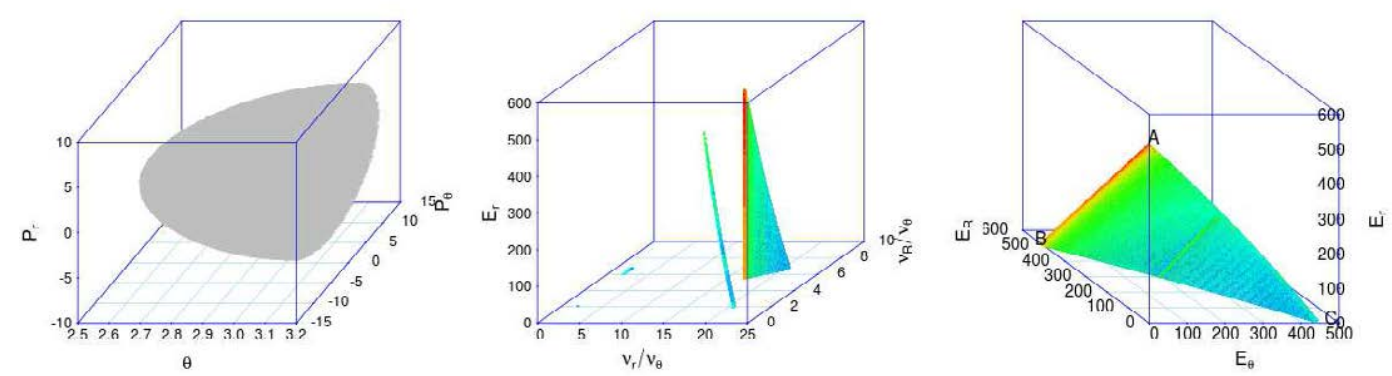

Figura 7.30: Grupo (g). Órbitas ligadas a la resonancia $(2,-6,1)$.

Las orbitas que forman parte de este grupo son la practica totalidad de las estudiadas en esta energía (98\% del total de la muestra). Las frecuencias de sus modos de vibración estan en la relación de resonancia $(1,-3,1 / 2)$. El triángulo $A B C$ citado se corresponde con el interior del triangulo $A B C$ del mapa $M E M_{3 D}(7.5$, a la derecha), por tanto las órbitas de consideradas tienen energía cinética en todos los modos de vibración.

Para efectuar un análisis detallado hemos escogido un grupo de órbitas con estas caracteristicas que pasamos a analizar a continuación:

- órbita $g 1$ (véase la figura 7.19)

- órbita $g 2$ (véase la figura 7.20)

- órbita $g 3$ (véase la figura 7.21) 
(a)

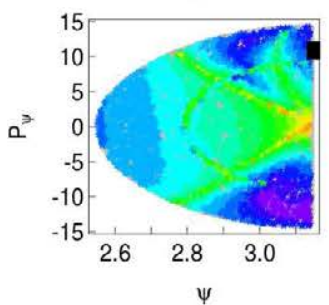

(d)

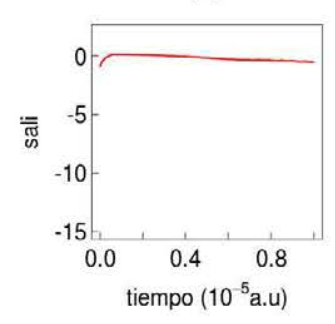

(g)

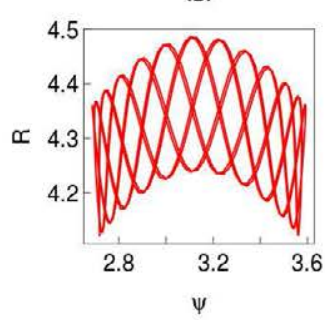

(j)

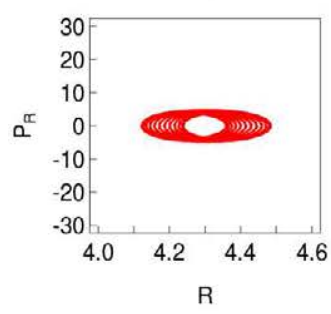

(m)

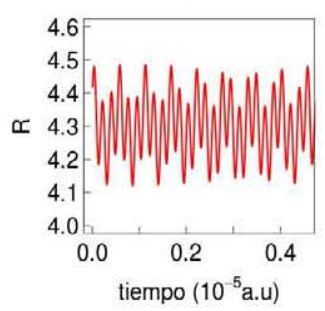

(b)

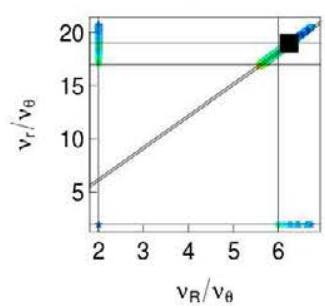

(e)

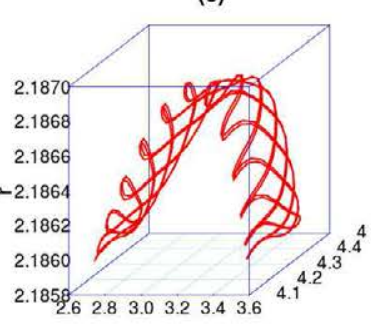

$\theta$

(h)

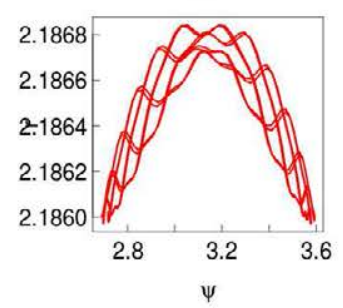

(k)

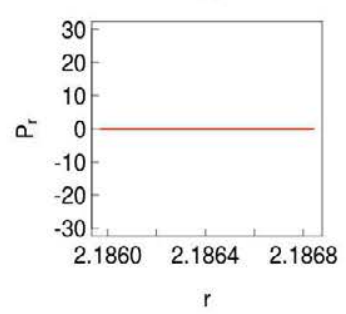

(n)

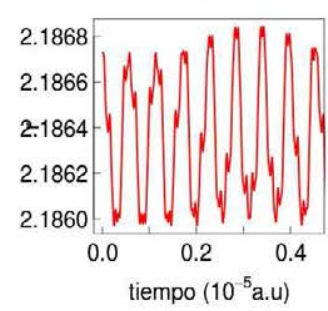

(c)

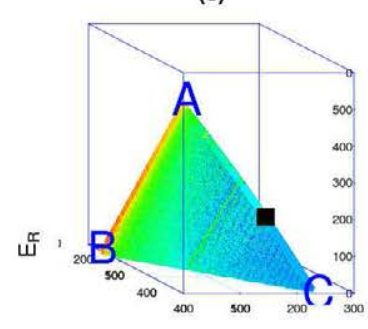

(f) $E_{\theta}$

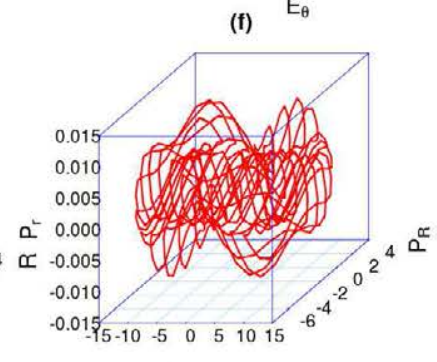

$\mathrm{P}_{\theta}$

(i)

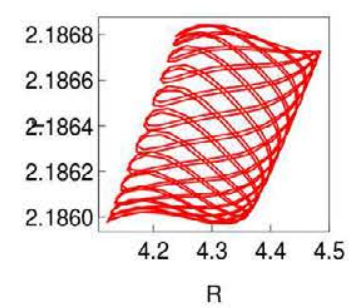

I

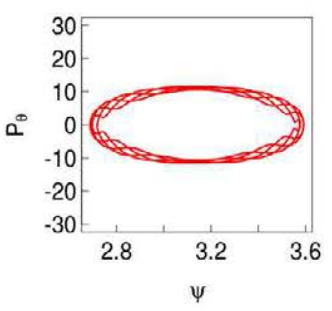

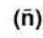

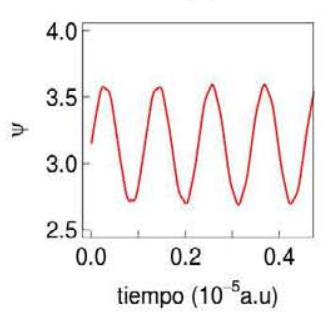

Figura 7.31: Descripción de la órbita g1. 
(a)

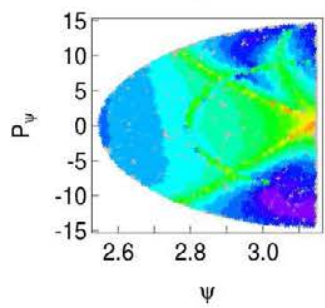

(d)

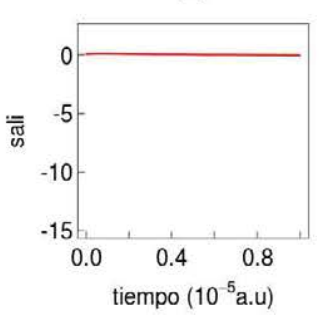

(g)

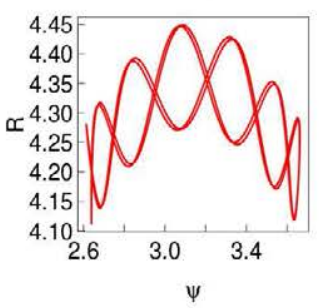

(j)

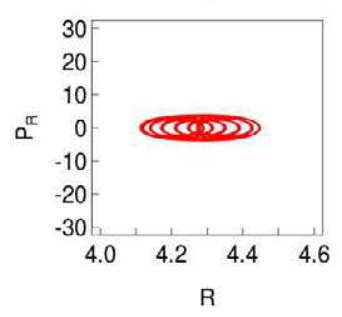

(m)

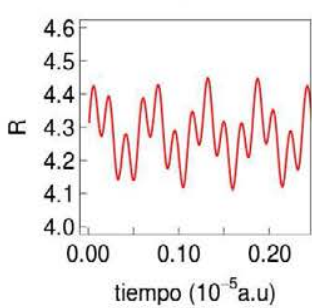

(b)

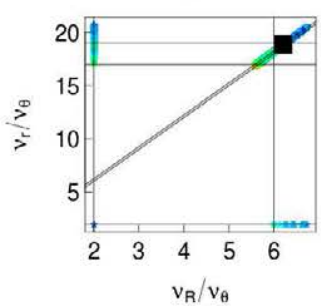

(e)

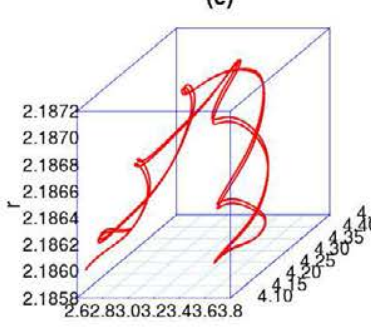

$\theta$

(h)

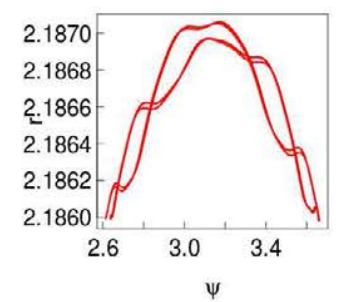

(k)

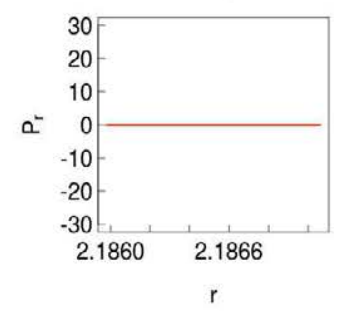

(n)

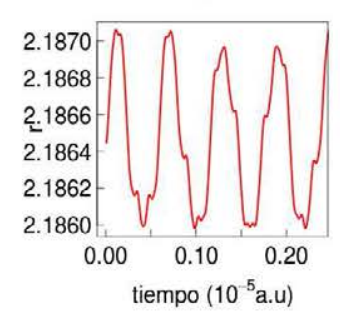

(c)

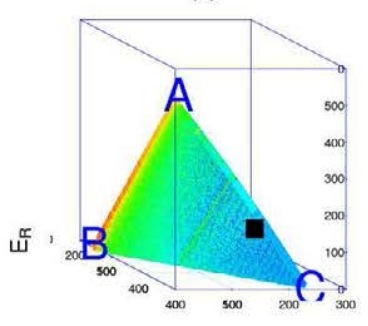

(f) $E_{\theta}$

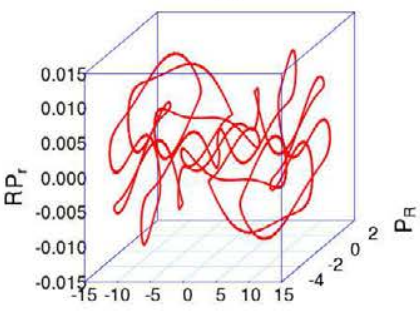

$\mathrm{P}_{\theta}$

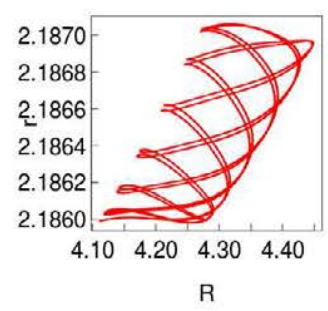

I

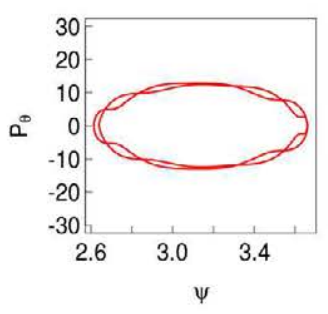

(n)

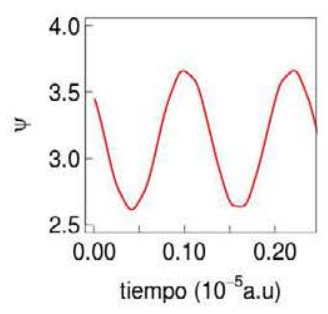

Figura 7.32: Descripción de la órbita g2. 
(a)

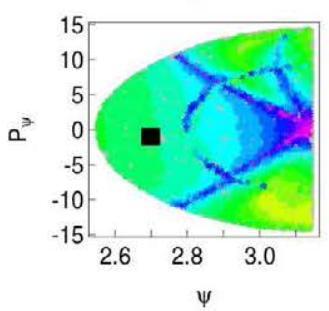

(d)

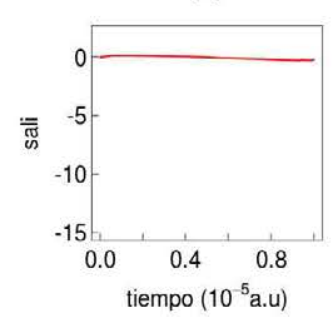

(g)

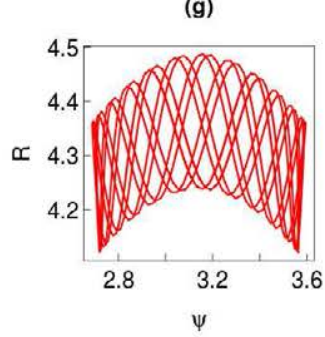

(j)

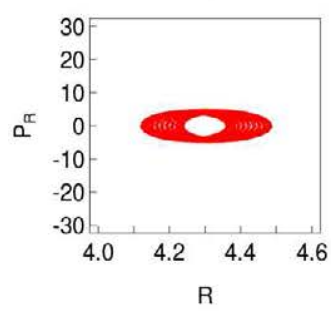

(m)

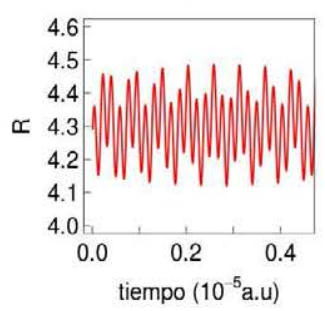

(b)

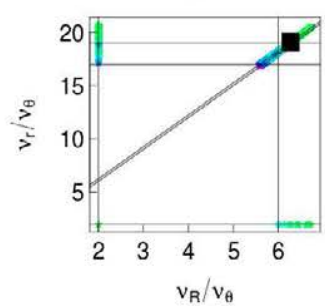

(e)

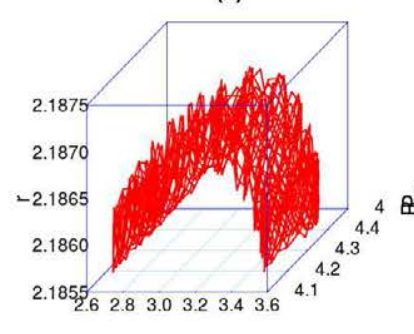

$\theta$

(h)

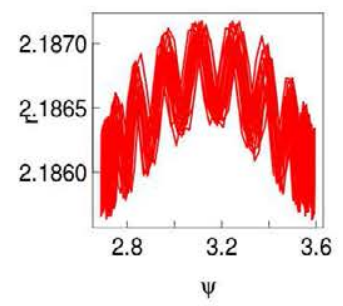

(k)

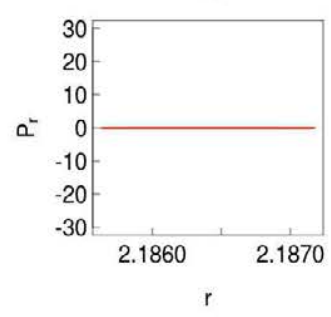

(n)

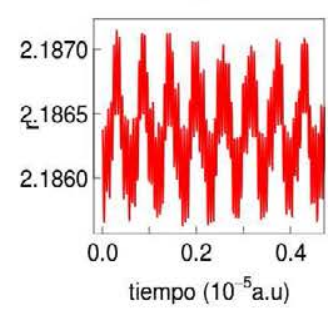

(c)

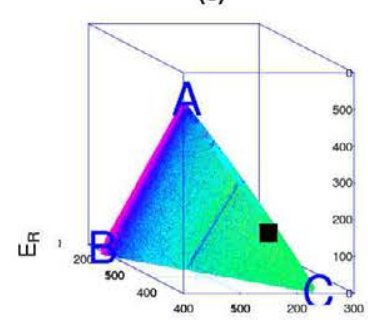

(f) $E_{\theta}$

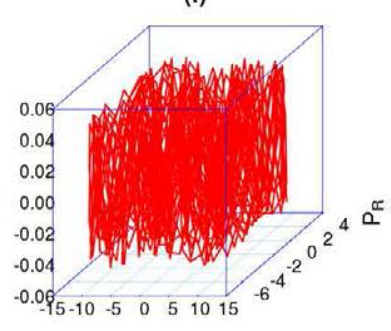

$\mathrm{P}_{\theta}$

(i)

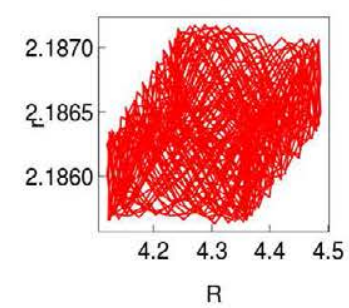

I

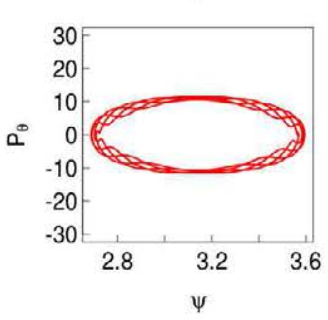

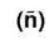

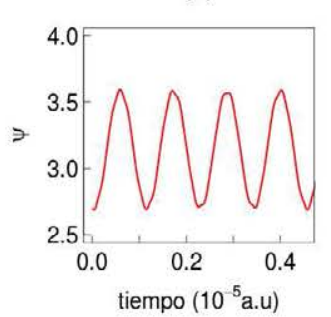

Figura 7.33: Descripción de la órbita g3. 

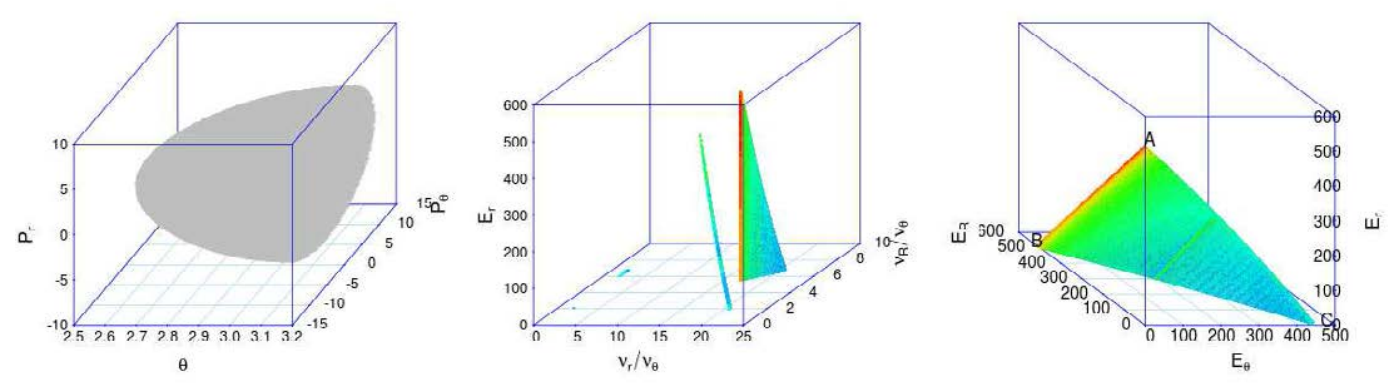

Figura 7.34: Grupo (h). Órbita en la resonancia $(1,1,-2)$.

En este grupo analizamos una unica órbita con la relación de frecuencias $(1,1,-2)$. Se observa en el mapa $M F E_{3 D}$ como un punto $H$ aislado que corresponde la órbita citada. Dicha orbita esta en el entono del punto $C$ del mapa $M E M_{3 D}$ 
(a)

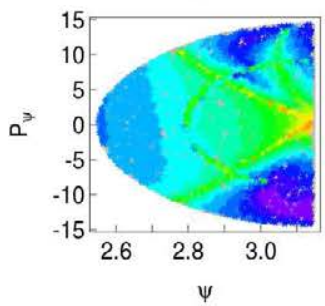

(d)

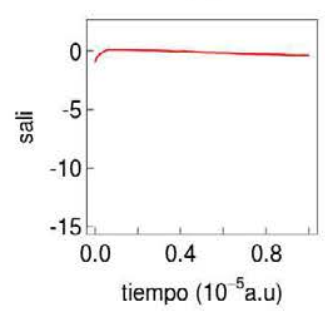

(g)

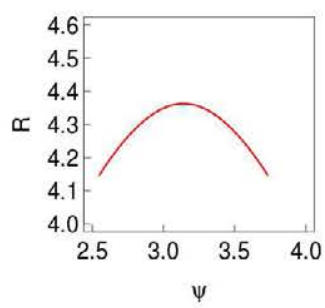

(j)

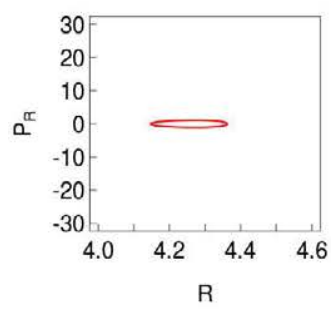

(m)

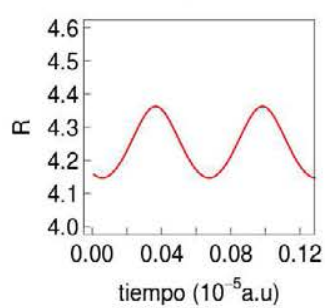

(b)

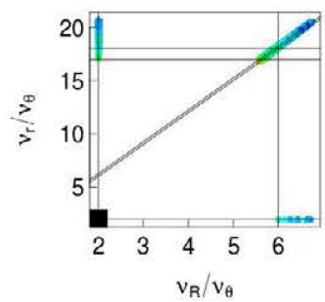

(e)

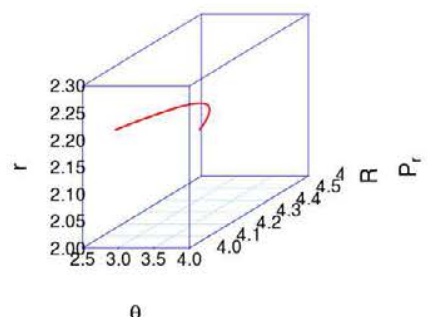

(h)

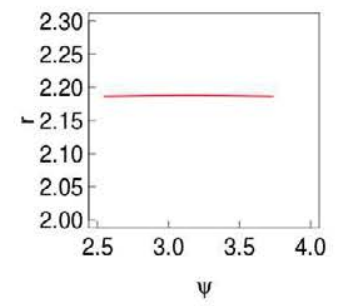

(k)

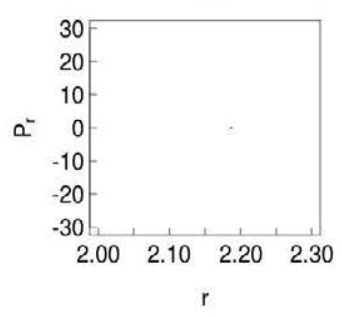

(n)

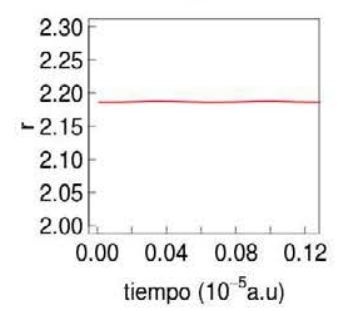

(c)

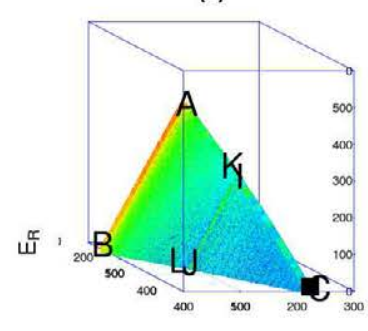

(f)

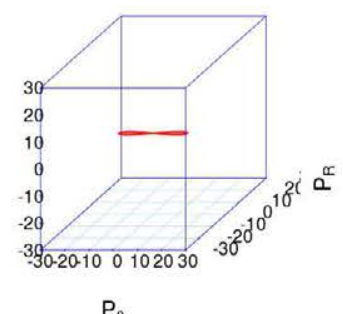

(i)

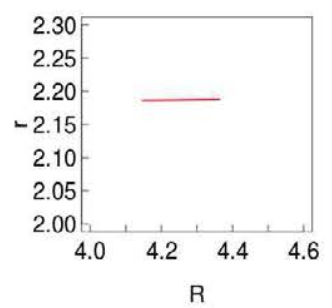

I

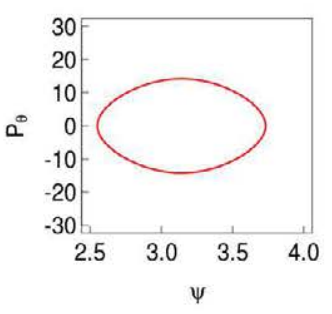

(ท)

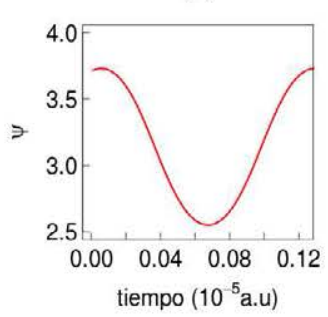

Figura 7.35: Descripción de la órbita h1. 


\subsection{SUMARIO.}

En este capítulo hemos analizado el sistema molecular $\operatorname{LiNC} / \operatorname{LiCN}$ a la energía de $512 \mathrm{~cm}^{-1}$. Se trata de un nivel muy bajo de la energía vibracional que nos garantiza el que todas las órbitas que analizamos sean en la práctica regulares. Con el fin de representar los diagramas correspondientes a los mapas $S M_{3 D}, M F E_{3 D}$ y $M E M_{3 D}$ hemos generado un gran número de condiciones iniciales (700.794) con las que hemos propagado las correspondientes órbitas.

En el mapa $S M_{3 D}$ las órbitas se agrupan en un semielipsoide de ejes $\psi \in[2,5, \pi] \mathrm{rad}, P_{r} \in[-10,10]$ u.a y $P_{\psi} \in[-15,15]$ u.a. Cortando dicho semielipsoide con planos formados por órbitas con valores de $P_{r}$ característicos, se observa la existencia de cierta estructura interior en el mismo, siendo reconocibles zonas más estables como las situadas en el entorno del pozo del potencial $\psi=\pi$, la resonancia $(1,0,-6)$ y las zonas de estabilidad aproximadamente lineales que parten del pozo de potencia $\psi=\pi$ hacia la frontera.

Analizando el mapa $M F E_{3 D}$ se comprueba que las órbitas estudiadas se agrupan en un triángulo plano $A B C$, dos segmentos $D E$ y $F G$ y un punto aislado $H$ que corresponden a grupos de órbitas caracterizadas por tener relaciones de resonancia específicas. Se puede comprobar que la mayor parte de las órbitas (órbitas en posición general) se sitúan en el interior del triángulo $A B C$. Se trata de órbitas que tienen energía cinética en todos los modos de vibración con una relación de frecuencias $(2,-6,1)$. Los otros grupos de órbitas corresponden a otras relaciones de frecuencia y son mucho más minoritarias.

$\mathrm{Al}$ representarlas en función a la energía cinética media de sus modos de vibración en $R, r$, y $\theta$ se observa que las órbitas se agrupan en un triángulo $A B C$ del mapa $M E M_{3 D}$ formado por los segmentos $\overline{A B}, \overline{B C}$ y $\overline{A C}$ de las órbitas que carecen de energía cinética en $\theta, R$ y $r$ respectivamente. Se detecta la existencia de resonancias entre los modos $R$ y $\theta$, entre $r$ y $\theta$ y entre $r$ y $R$. Las órbitas en estas resonancias se sitúan a lo largo de segmentos de recta aproximadamente paralelos al lado $\overline{A B}$.

La mayor parte de las óbitas estables se acumulan en el entorno de la línea $\overline{A B}$ que representa los puntos en los que la energía atribuida al modo $\theta$ es menor y en general va disminuyendo a medida de que nos acerquemos bien a una resonancia (como la $(1,0,-6)$ ) bien al vértice $C$ donde la energía cinética en $\theta$ es mínima. 


\section{Parte IV}

\section{CONCLUSIONES}





\section{Capítulo 8}

\section{Conclusiones}

A continuación se enumeran las conclusiones obtenidas tras el análisis de la estructura del espacio de fases del sistema molecular $\operatorname{LiNC} / \mathrm{LiCN}$ considerando los modelos de 2D y 3D mediante los diferentes mapas de SALI desarrollados en esta tesis y sus correspondientes visualizaciones.

\section{Respecto al estudio del sistema 2D}

1. Los mapas de SALI-SSP 2D ofrecen unos resultados que concuerdan con los obtenidos con las Superficies de Sección de Poincare (SSP), proporcionando información dinámica adicional, ya que permite distinguir entre diferentes grados de caoticidad, según el valor mínimo de SALI obtenido para un periodo $T$ determinado $\left(m S A L I_{T}\right)$.

2. La evolución temporal de SALI nos permite observar los periodos de tiempo en los que una trayectoria caótica se ve influenciada por las estructuras regulares del espacio de fases. También permite distinguir trayectorias regulares con diferentes características dinámicas, pudiendo clasificar las trayectorias periódicas en estables o inestables según el comportamiento temporal de SALI.

3. El mapa Diagrama de Bifurcación-SALI permite poner de manifiesto como la dinámica es regular para trayectorias con condiciones iniciales con valores extremos de la coordenada $R$. Así mismo hemos observado como en las bifurcaciones el valor de $m S A L I_{T}$ decrece sensiblemente

4. Mediante los mapas de SALI podemos distinguir tres regiones en el espacio de fase del sistema $\operatorname{LiNC} / \operatorname{LiCN} 2 \mathrm{D}$ : 
- Región de Movimiento Regular: Compuesta por trayectorias que están organizadas en toros KAM alrededor de los pozos de potencial correspondientes a los dos isómeros y por zonas de cadenas de islas.

- Región de Caos Suave: Compuesta por trayectorias caóticas que están influenciadas por la frontera de las regiones regulares mencionadas antes y otras estructuras casi regulares, como los cantoros.

- Región de Caos Fuerte: Compuesta por trayectorias caóticas que se mueven libremente, recorriendo la parte ergódica del espacio de fase.

5. La proporción entre los volumenes de las tres regiones citadas anteriormente cambia con el incremento de la energía, pasando globalmente de más regular a más caótica cuando la energía total del sistema aumenta.

6. A energías muy bajas, el $100 \%$ del espacio de fases corresponde a movimiento regular, apareciendo zonas significativas de caos a partir de aproximadamente $E=1600 \mathrm{~cm}^{-1}$

7. El caos suave solo existe significativamente en el intervalo $E=1600-$ $2900 \mathrm{~cm}^{-1}$. Comienza a crecer a un valor ligeramente inferior de energía que el caos fuerte y una vez alcanzado un máximo del $21.08 \%$ en $E=$ $1850 \mathrm{~cm}^{-1}$ cae rápidamente a cero.

8. Según aumenta la energía la zona regular va disminuyendo hasta alcanzar un valor asintótico del $6 \%$ que se mantiene incluso a muy altas energías.

\section{Respecto al estudio del sistema 3D}

1. Hemos representado la generalización del mapa Diagrama de Bifurcación-SALI mediante una visualización 4D (en la que la cuarta dimensión es el valor de $m S A L I_{T}$ ). Hemos observado que, de manera análoga al sistema $2 \mathrm{D}$, la mayor parte de la zona regular se sitúa en la capa de valores extremos de la coordenada $R$, apareciendo zonas regulares internas que corresponden a las diferentes ramas del diagrama de bifurcaciones.

2. Hemos representado mapas de SALI mediante visualizaciones 4D para diferentes energías. Mediante estas visualizaciones y diferentes cortes hemos podido caracterizar completamente la estructura del espacio de 
fases del sistema $\mathrm{LiNC} / \mathrm{LiCN}$ en 3D para distintos valores de la energía vibracional total, comprobando como, de manera análoga al sistema $2 \mathrm{D}$, podemos distinguir las tres regiones de regularidad, caos suave y caos fuerte.

3. Para energías bajas el movimiento es regular en todo el espacio de fases accesible. El corte con el plano $P_{r}=0$, es decir cuando las condiciones iniciales tienen momento de la tercera coordenada iguales a cero (condiciones análogas al sistema 2D), muestra la zona de regularidad al rededor del pozo más estable y dos zonas de cadenas de islas. Este corte es muy similar al mapa de SALI 2D a esta energía

4. Para energías intermedias observamos dos zonas de regularidad correspondientes a los dos pozos y las diferentes zonas de caos suave y caos fuerte. Al aumentar el valor de $\left|P_{r}\right|$ las zonas de caos van desapareciendo, debido a que la energá disponible para la coordenada angular $\theta$ y para la coordenada $R$ disminuye.

5. Al aumentar más la energía, el caos suave desaparece, manteniéndose las zonas regulares alrededor de los pozos del potencial, en zonas de cadenas de islas y para valores de $\left|P_{r}\right|$ cerca de su máximo para cada valor de la energía, dado que la energía disponible a los otros modos de vibración es menor, mostrando que el movimiento en esta coordenada angular está desacoplado del resto incluso para valores muy altos de la energía vibracional total del sistema.

6. Los porcentajes de los volúmenes correspondientes a cada una de las tres zonas en función de la energía tienen un comportamiento análogo al del sistema 2D, con un intervalo de energías en el que el caos suave es significativo y con un valor asintótico constante de zona regular, que se alcanza para valores mayores de la energía en el caso del sistema 3D

7. Hemos comprobado como el valor asintótico de caos/regularidad depende de la elección del valor de $P_{r}$ en las condiciones iniciales. Así si $P_{r}$ es mayor, la proporción de trayectorias regulara es mayor. Esto es una clara evidencia de que el movimiento en la coordenada $r$ está muy desacoplado, de forma que el porcentaje de caos depende de la energía vibracional disponible para las coordenadas del sistema 2D.

8. Las visualizaciones $4 \mathrm{D}$ del mapa de frecuencias con SALI nos han permitido comprobar que las órbitas regulares se agrupan a lo largo de lineas de resonancia en el espacio de frecuencias. A medida de que la 
energía crece, crece también el número de lineas de resonancia accesibles. Las orbitas caóticas se sitúan mayoritariamente en el entorno de la intersección de lineas de resonancia. Ademas se pone de manifiesto que el movimiento de las trayectorias caóticas no es completamente érgodico sino que éste está influenciado por las resonancias que hacen que las trayectorias caóticas pasen periodos de tiempo atraídas o atrapadas por las mismas.

9. Las visualizaciones $4 \mathrm{D}$ del mapa de energía media con SALI evidencian que las órbitas regulares se agrupan en figuras planas, mientras que las órbitas caóticas se esparcen libremente por el espacio de energía.

10. En el estudio del sistema molecular $\operatorname{LiNC} / \operatorname{LiCN}$ a un nivel de energía muy bajo, en el que se puede suponer la inexistencia de órbitas caóticas, hemos comporbado como en el mapa de SALI las órbitas se agrupan en un semielipsoide de ejes $\psi \in[2,5, \pi] \mathrm{rad}, P_{r} \in[-10,10]$ u.a y $P_{\psi} \in[-15,15]$ u.a.; en el mapa de frecuencias con SALI las órbitas se agrupan en tres regiones bien definidas: un plano de forma triangular, dos segmentos y un punto aislado que corresponden a grupos de órbitas caracterizadas por tener relaciones de resonancia específicas; y en el mapa de Energía media con SALI, las órbitas se agrupan en un triángulo limitado por tres segmentos correspondientes con de las órbitas sin energía cinética en $\theta, R$ y $r$ respectivamente. Las diferentes resonancias entre los modos de vibración aparecen como segmentos paralelos a cada uno de los lados del triángulo. 


\section{Bibliografía}

[1] H.Golstein, Mecánica Clásica Ed. Reverte, (2005).

[2] F.R. Gantmayer, Mecánica Analítica URSS, (2003).

[3] V.I. Arnol'd, Metodos Matematicos de la Mecánica Clásica Ed.Paraninfo, (1978).

[4] Robert L. Devaney ,An Introduction to Dynamical Systems, Addison -Wesley, (1989).

[5] Á. Jorba and J. Villanueva. On the persistence of lower dimensional invariant tori under quasi-periodic perturbations. J. Nonlinear Sci., 7:427473, 1997.

[6] Á. Jorba and C. Simó. Efective stability for periodically perturbed Hamiltonian systems. In J. Seimenis, editor, Hamiltonian Mechanics: Integrability and Chaotic Behaviour, volume 331 of NATO Adv. Sci. Inst. Ser. B Phys., Held in Torun, Polland, 28 June 2 July 1993. Plenum, New York, 1994, pp. 245-252

[7] Á. Jorba and J. Villanueva. On the normal behaviour of partially elliptic lower dimensional tori of Hamiltonian systems. Nonlinearity, 10:783822, 1997.

[8] A. N. Kolmogorov, Dokl. Akad. Nauk, SSSR, 98, 527 (1954).

[9] R. de la Llave, Introduction to KAM theory, in Computational Physics, World Scientific, River Edge, NJ, 1992, pp. 73-105.

[10] R. de la Llave, A. González, Á. Jorba, J. Villanueva, KAM theory without action-angle variables Nonlinearity, 18 (2) (2005), pp. 855-895.

[11] R de la Llave, A tutorial on KAM theory in Proceedings of Symposia in Pure Mathematics 69, pp. 175-296. 
[12] A.J. Lichtemberg, M.A. Lieberman Regular and Chaotic Dynamics (1983).

[13] V. I. Oseledets, A multiplicative ergodic theorem: Lyapunov characteristic numbers for dynamical systems.Trans.Moscow Math.Soc. 19 (1968), 197-231.

[14] J. Laskar, The chaotic motion of the solar system: A numerical estimate of the size of the chaotic zones, Icarus 88, 266 (1990).

[15] J. Laskar, Physica D 67, 257 (1993).

[16] J.C. Losada, C. G. Giralda, R. M. Benito, and F. Borondo, AIP Conf. Proc. 905, 249 (2007).

[17] J. C. Losada, J. M. Estebaranz, R. M. Benito, F. Borondo, Local frequency analysis and the structure of classical phase space of the LiNC/LiCN molecular system. J. Chem. Phys. 108 (1), 1 (1998)

[18] J. C. Losada, R. M. Benito, and F. Borondo, Eur. Phys. J.: Special Topics 165, 183 (2008).

[19] Skokos Ch., Antonopoulos Ch., T.C. Bountis, and M. N. Vrahatis, J .Phy. A: Math. Gen. 34, 10029 (2001).

[20] Skokos Ch., Ch. Antonopoulos, T.C. Bountis, and M. N. Vrahatis, Prog. Theor. Phys. Supp. 150, 439 (2003).

[21] Skokos Ch, Ch. Antonopoulos, T.C. Bountis, and M. N. Vrahatis, J. Phys. A: Math. Gen. 37, 6269 (2004).

[22] Skokos Ch., Bountis T.C., Complex Hamiltonian Dynamics (Springer Series in Synergetics, New York, 2012).

[23] Bountis, T.C. and Skokos, Ch.:Nucl. Instr. Meth. Phys. Res. Sect. A 561,173 (2006)

[24] Skokos Ch., T. C. Bountis, and Ch. Antonopoulos, Physica D 231, 30 (2007).

[25] Skokos, Ch., Bountis, T.C. and Antonopoulos, Ch.Detecting chaos, determining the dimensions of tori and predicting slow diffusion in FermiPasta-Ulam lattices by the generalized alignment index method. Eur. Phys. J. Spec. Top. 165, 5 (2008) 
[26] Skokos, Ch., Bountis, T.C., Antonopoulos, Ch.Detecting chaos, determining the dimensions of tori and predicting slow diffusion in Fermi-PastaUlam lattices by the generalized alignment index method. Eur. Phys. J. Spec. Top. 165, 5 (2008)

[27] Manos, T., Skokos, Ch., Bountis, T. Application of the Generalized Alignment Index (GALI) method to the dynamics of multi-dimensional symplectic maps. In: Chandre C., Leoncini X. and Zaslavsky G. (eds.) Chaos, Complexity and Transport: Theory and Applications. Proceedings of the CCT 07, World Scientific, pp. 356-364 (2008)

[28] P. Benitez, J. C. Losada, R. M. Benito, and F. Borondo, Analysis of the Full Vibrational Dynamics of the LiNC/LiCN Molecular System in Progress and Challenges in Dynamical Systems, S. Ibañez, J. S. Pérez del Rio, A. Pumariño and J. A. Rodríguez (eds.), Springer Proceedings in Mathematics \& Statistics, vol. 54 (Springer-Verlag, Berlin, 2013).

[29] P. Benitez, J. C. Losada, R. M. Benito, and F. Borondo. Using the small alignment index chaos indicator to characterize the vibrational dynamics of a molecular system: LiNC-LiCN, PHYSICAL REVIEW E 92, 042918 (2015).

[30] J. Tennyson and B. T. Sutcliffe, J. Chem. Phys. 77, 4061 (1982)

[31] R. Essers, J. Tennyson, and P. E. S. Wormer, Chem. Phys. Lett. 89, 223 (1982).

[32] W. Quapp and D. Heidrieh, Analysis of the concept of minimum energy path on the potential energy surface of chemically reacting systems. Theoret. Chim. Acta (Bed.) 66, 245-260 (1984)

[33] F. Borondo, J. M. Gomez-Llorente and R. M. Benito, Laser Chem. 12, 85 (1992); F. Borondo and R. M. Benito, in Frontiers of Chemical Dynamics, NATO ASI Series C, edited by E. Yurtsever (Kluwer, Dordrecht, 1995); R. Prosmiti, S. C. Farantos, R. Guantes, F. Borondo, and R. M. Benito, J. Chem. Phys. 104, 2921 (1996); F. J. Arranz, F. Borondo, and R. M. Benito, J. Chem. Phys. 104, 6401 (1996); ibid. 107, 2395 (1997); J. Mol. Struct. (Theochem) 426, 87 (1998); R. M. Benito and F. Borondo, in Ref. [47], pp. 310-313; F. Borondo, E. G. Vergini, A.A. Zembekov and R.M. Benito, J. Chem. Phys. 12211101 (2005).

[34] R.M. Benito, F. Borondo, J.H. Kim, B.G. Sumpter, G.S. Ezra, Chem. Phys. Lett. 161, 60 (1989). 
[35] F. J. Arranz, F. Borondo, and R. M. Benito, Phys. Rev. Lett. 80, 944 (1998).

[36] P. Garcia-Muller R. M. Benito, R. Hernandez, and F. Borondo, J. Chem. Phys. 141, 074312 (2014).

[37] F. Borondo, A. A. Zembekov, and R. M. Benito, Chem. Phys. Lett. 246, 421 (1995); J. Chem. Phys. 105, 5068 (1996); ibid. 107, 7934 (1997).

[38] V. Afraimovich and G. M. Zaslavsky, in Kinetics and Nonlinear Dynamics in Fluids and Plasmas (Springer, Berlin Heidelberg, 1998), pp. 59-82.

[39] M.J Davis and S.K.Gray, J. Chem. Phys. 845389 (1986)

[40] R. Prosmiti, S.C. Farantos,R. Guantes, F. Borondo and R. M. Benito J.Chem. Phys. 1042921 (1996)

[41] F. J. Arranz, R.M. Benito and F. Borondo, J. Chem. Phys. (2005); 123(13):134305. DOI: 10.1063/1.2039767

[42] E. Hairer, S.P. Norsett and G. Wanner, Solving Ordinary Differential Equations I. Nonstiff Problems.,Springer Verlag (1993.)

[43] E. L. Sibert, W. P. Reinhardt, and J. T Hynes, J. Chem. Phys. 77, 3583 (1982).

[44] S. Keshavamurthy, in Adv. Chem. Phys. 153, eds S. A. Rice and A. R. Dinner (John Wiley \& Sons, Hoboken, 2013).

[45] M. Gruebele and P. G. Wolynes, Acc. Chem. Res. 37, 261 (2004).

[46] A. Zewail (ed), The chemical bond: Structure and dynamics (Academic Press, San Diego, 1992).

[47] C. Simó (ed), Hamiltonian systems with three or more degrees of freedom, NATO ASI Series C (Kluwer, Dordrecht, 1999).

[48] C. Froeschle and E. Lega, Celest. Mech. Dyn. Astr. 78, 167 (2000).

[49] R. Barrio, Chaos, Solitons \& Fractals, 25, 711 (2005).

[50] L. E. Reichl, The Transition to Chaos in Conservative Classical Systems: Quantum Manifestations (Springer, New York, 1992). 
[51] Ya. G. Sinai, Introduction to ergodic theory (Princeton Univ. Press, Princeton, 1977).

[52] Voglis, N., Harsoula, M., Contopoulos, G. Orbital structure in barred galaxies Mon. Not. R. Astron. Soc. 381, 757 (2007)

[53] Voyatzis, G. Chaos, order, and periodic orbits in 3:1 resonant planetary dynamics. Astroph. J. 675, 802 (2008)

[54] Bountis, T., Papadakis, K.E. The stability of vertical motion in the $N$ body circular Sitnikov problem. Cel. Mech. Dyn. Astr. 104, 205 (2009)

[55] Manos, T., Athanassoula, E.Regular and chaotic orbits in barred galaxies I. Applying the SALI/GALI method to explore their distribution in several models. Mon. Not. R. Astron. Soc. 415, 629 (2011)

[56] Manos, T., Bountis, T., Skokos, Ch. Interplay between chaotic and regular motion in a time dependent barred galaxy model. J. Phys. A: Math. Theor., 46, 254017 (2013)

[57] Maffione, N.P., Darriba, L.A., Cincotta, P.M., Giordano, C.M. A comparison of different indicators of chaos based on the deviation vectors: application to symplectic mappings. Cel. Mech. Dyn. Astron. 111, 285 (2011)

[58] Zotos, E.E., Caranicolas, N.D.Order and chaos in a new 3D dynamical model describing motion in non-axially symmetric galaxies. Nonlinear Dyn. 74, 1203 (2013)

[59] Zotos, E.E.Classifying orbits in galaxy models with a prolate or an oblate dark matter halo component. Astron. Astroph. 563, A19 (2014)

[60] N. Kyriakopoulos, V. Koukouloyannis, C. Skokos, and P. Kevrekidis, Chaotic behavior of three interacting vortices in a confined Bose-Einstein condensate Chaos 24, 024410 (2014).

[61] Chr. Antonopoulos,V. Basios and Tassos Bountis, Weak chaos and the 'melting transition'in a confined microplasma system , PRE 81 , 016211

[62] P. Le Calvez and J. Wang, Proc. Amer. Math. Soc. 138, 703 (2010).

[63] B. V. Chirikov, Phys. Rep. 52, 265 (1979)

[64] G. Murgida, D. Wisniacki, P. Tamborenea, and F. Borondo, Chem. Phys. Lett. 496, 356 (2010); G. Murgida, F.J. Arranz, and F. Borondo, J. Chem. Phys. (submitted). 
[65] P.M. Poggi, F.J. Arranz, R.M. Benito, F. Borondo, and D.A. Wisniacki, Phys. Rev. A 90, 062108 (2014).

[66] L.A. Pellouchoud and E.J. Reed, Phys. Rev. A 91, 052706 (2015).

[67] J.C. Butcher, Numerical Methods for Ordinary Differential Equations, Wiley \& Sons,New York (2008)

[68] C.W. Gear, Numerical initial value problems in ordinary differential equations, Prentice Hall (1971).

[69] R. Seydel, Practical Bifurcation and Stability Analysis, Interdisciplinary Applied Mathematics 5 (Springer-Verlag, Berlin, 2010). 


\section{Apéndice A}

\section{Energía Media}

En este apéndice presentamos las expresiones análiticas para el cálculo de la energía media de los distinto modos de vibración del $\operatorname{LiNC} / \operatorname{LiCN} 3 \mathrm{D}$.

Dedicaremos la primera sección (A.1) a la obtención de las energías medias mientras que las siguientes están dedicadas a la relación de la energia con la con la acción (A.2) y finalmente establecemos la unicidad de los resultados (A.3).

\section{A.1. Cálculo de la Energía Media}

Partimos del Hamiltoniano:

$$
H=\frac{P_{R}^{2}}{2 m_{1}}+\frac{P_{r}^{2}}{2 m_{2}}+\frac{P_{\theta}^{2}}{2}\left(\frac{1}{m_{1} R^{2}}+\frac{1}{m_{2} r^{2}}\right)+V_{t}(R, r, \theta) .
$$

Dado que $\mathrm{H}$ no depende explicitamente del tiempo sabemos que $\frac{\partial H}{\partial t}=$ $\frac{d H}{d t}=0$ por tanto $H$ es una constante que equivale a la energía del sistema. Por tanto podemos afirmar que $H=E=$ Constante.

Fijemos T un tiempo arbitrariamente grande. Integando la expresión A.1 en el intervalo $(0, T)$ tenemos:

$$
\int_{0}^{T} H d t=\int_{0}^{T} \frac{P_{R}^{2}}{2 m_{1}}+\frac{P_{r}^{2}}{2 m_{2}}+\frac{P_{\theta}^{2}}{2}\left(\frac{1}{m_{1} R^{2}}+\frac{1}{m_{1} r^{2}}\right)+V_{t}(R, r, \theta) d t=E T
$$

O lo que es lo mismo: 
$\int_{0}^{T} \frac{P_{R}^{2}}{2 m_{1}} d t+\int_{0}^{T} \frac{P_{r}^{2}}{2 m_{2}} d t+\int_{0}^{T} \frac{P_{\theta}^{2}}{2}\left(\frac{1}{m_{1} R^{2}}+\frac{1}{m_{1} r^{2}}\right) d t+\int_{0}^{T} V_{t}(R, r, \theta) d t=E T$

Consideremos las expresiones:

$$
\begin{gathered}
E_{R} \equiv \frac{1}{T} \int_{0}^{T} \frac{P_{R}^{2}}{2 m_{1}} d t \\
E_{r} \equiv \frac{1}{T} \int_{0}^{T} \frac{P_{r}^{2}}{2 m_{2}} d t \\
E_{\theta} \equiv \frac{1}{T} \int_{0}^{T} \frac{P_{\theta}^{2}}{2}\left(\frac{1}{m_{1} R^{2}}+\frac{1}{m_{1} r^{2}}\right) d t .
\end{gathered}
$$

Se trata de cantidades que tienen la dimensión de una energía. Por otra parte son integrales definidas con un integrando positivo y estan acotadas (por $E$ ). Por tanto deben converger a un valor definido en cada una de las órbitas.

\section{A.2. Relación entre la energía media y la ac- ción}

Impongamos ahora la hipotesis de que el movimiento que estamos estudiando sea regular. Por el teorema de Arnol'd-Liuoville esto significa que el movimietp se dsarrolla en un n-toro.

Recordemos la definicion de la acción:

$$
I_{x} \equiv \oint P_{x} d x
$$

Donde llamamos $x$ a cualquiera de las variables que estamos manejando: $R ; r ; \theta$ y efectuamos la integraci a lo largo de uno de los circuitos elementales que definen un toro.

Efectuando un cambio de variable tenemos trivialmnte

$$
I_{x} \equiv \oint P_{x} d x=\int_{0}^{T_{0}} P x \frac{d x}{d t} d t ;=; \int_{0}^{T_{0}} P_{x} \dot{x} d t
$$

Donde $T_{0}$ es el periodo.

En el caso de una orbita regular sabemos que se trata de un invariante.

Recordando las ecuaciones de Hamilton, por ejemplo para $R$, tenemos: 


$$
I_{R}=\int_{0}^{T_{0}} P_{R} \dot{R} d t=\int_{0}^{T_{0}} P_{R} \frac{P_{R}}{m_{1}} d t=\int_{0}^{T_{0}} \frac{P_{R}^{2}}{m_{1}} d t
$$

Como $T$ es arbitrariamente grande, siempre podemos hacer $T=n T_{0}+\epsilon$ con $n>>1$ y $\epsilon<T_{0}$.

Por otra parte tenemos:

$$
2 E_{R}=; \frac{1}{n T_{0}+\epsilon}\left[\int_{0}^{T_{0}} \frac{P_{R}^{2}}{m_{1}} d t+\int_{T_{0}}^{2 T_{0}} \frac{P_{R}^{2}}{m_{1}} d t+\ldots+\int_{n T_{0}}^{T} \frac{P_{R}^{2}}{m_{1}} d t\right]
$$

Para $T$ muy grande

$$
2 E_{R}=; \frac{1}{n T_{0}+\epsilon}\left[n I_{R}+\int_{n T_{0}}^{T} \frac{P_{R}^{2}}{m_{1}}\right] \sim \frac{I_{R}}{T_{0}}
$$

Recordando finalmnte que $T_{0}=\frac{2 \pi}{\nu_{R}}$ tenemos

$$
E_{R} \sim \nu_{R} I_{R}
$$

Y de forma totalmente analoga se puede demostrar:

$$
\begin{aligned}
& E_{r} \sim \nu_{r} I_{r} \\
& E_{\theta} \sim \nu_{\theta} I_{\theta}
\end{aligned}
$$

\section{A.3. Unicidad de la energía media de las tra- yectorias}

Sea $\vec{z}_{0}(t)$ una orbita definida por la condición inicial $z_{0} \in \Omega_{3 D}$ en el espacio de fase.

Denominaremos trayectoria al conjunto de puntos del espacio de fase que recorre la órbita con independencia de su ley horaria. Sea $\vec{z}_{1}$ un punto cualquiera de la trayectoria, es decir $\vec{z}_{1}=\vec{z}\left(t_{1}\right)$ con $t_{1}>0$. Consideremos la orbita $\overrightarrow{z_{1}}(t)$ definida por tener la condición inicial vec $z_{1}$ en el espacio de fase.

\section{PROPOSICIÓN}


Sean $E_{R}\left(z_{0}\right), E_{r}\left(z_{0}\right)$ y $E_{\theta}\left(z_{0}\right)$ las energías medias correspondientes a la órbita $\vec{z}_{0}(t)$ y de forma análoga $E_{R}\left(z_{1}\right), E_{r}\left(z_{1}\right)$ y $E_{\theta}\left(z_{1}\right)$ las energías correspondientes a la órbita $\vec{z}_{1}(t)$. Entoces se verifica que:

$$
\begin{aligned}
& E_{R}\left(\vec{z}_{0}\right) \sim E_{R}\left(\vec{z}_{1}\right) \\
& E_{r}\left(\vec{z}_{0}\right) \sim E_{r}\left(\vec{z}_{1}\right) \\
& E_{\theta}\left(\vec{z}_{0}\right) \sim E_{\theta}\left(\vec{z}_{1}\right)
\end{aligned}
$$

Demostraremos esta proposición solo para $E_{R}$ siendo la demostracion para los otros modos de vibración totalmente análogo.

$$
\begin{aligned}
& E_{R} \equiv \frac{1}{T} \int_{0}^{T} \frac{P_{R}^{2}}{2 m_{1}} d t \\
& E_{R} \equiv \frac{1}{T} \int_{0}^{t_{1}} \frac{P_{R}^{2}}{2 m_{1}} d t+\frac{1}{T} \int_{t_{1}}^{T} \frac{P_{R}^{2}}{2 m_{1}} d t
\end{aligned}
$$

Sabemos que $\frac{1}{T} \int_{0}^{t_{1}} \frac{P_{R}^{2}}{2 m_{1}} d t \sim 0$ dado que asumimos que $T$ es tan grande como se quiera, por tanto

$$
\begin{aligned}
& E_{R}\left(\vec{z}_{0}\right) \equiv \frac{1}{T} \int_{t_{1}}^{T} \frac{P_{R}^{2}}{2 m_{1}} d t \equiv \frac{1}{T} \int_{0}^{T-t 1} \frac{P_{R}^{2}}{2 m_{1}} d s \sim E_{R}\left(\text { vec } z_{1}\right) \\
& E_{R}\left(\vec{z}_{0}\right) \sim E_{R}\left(\text { vec } z_{1}\right)
\end{aligned}
$$

Donde hemos efecurtado el cambio de variable $s=t-t_{1}$ y aprovechado el hecho de que $t$ sea una variable muda en A.4. 


\section{Apéndice B}

\section{Comportamiento Asintótico de SALI}

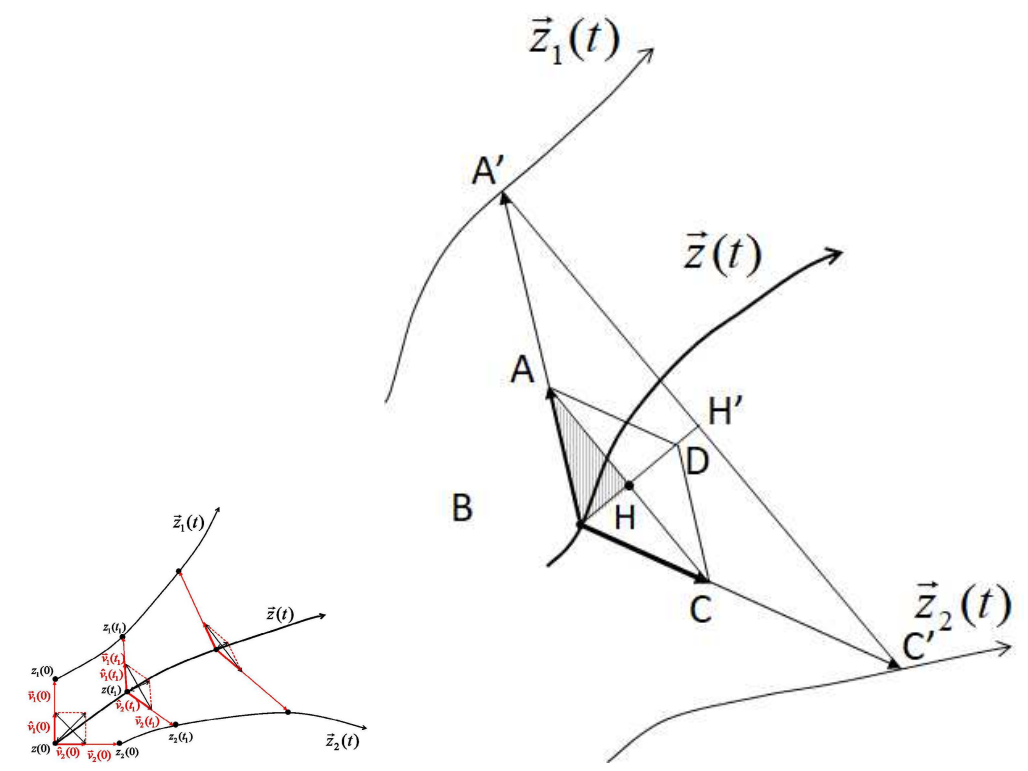

Figura B.1: Vectores de desviación. Detalle de la figura 3.4

En este apéndice analizaremos el comportamiento de $S A L I$ para un tiempo grande, es decir su comportamiento asintótico, en los casos de movimiento caótico y regular.

Para calcular el valor de SALI nos apoyaremos en la figura B.1 en la que 
se detalla la definición de los vectores de desviacióon que intervienen en la construción del indicador SALI. Llamemos $S$ al área del rombo $A B C D$ de la figura. De acuerdo con la geometría elemental, a partir de la semejanza de los triángulos $A B H$ y $A^{\prime} B H^{\prime}$ tenemos:

$$
\begin{aligned}
& \frac{|A H|}{|A B|}=\frac{\left|A^{\prime} H^{\prime}\right|}{\left|A^{\prime} B\right|} \\
& \frac{|B H|}{|A B|}=\frac{\left|B H^{\prime}\right|}{\left|A^{\prime} B\right|}
\end{aligned}
$$

Calculamos el area del rombo $A B C D$ :

$$
\begin{aligned}
& S=\left(\frac{1}{2}|A H||B H|\right) 4 \\
& S=2 \frac{\left|A^{\prime} H^{\prime}\right|}{\left|A^{\prime} B\right|} \frac{B H^{\prime} \mid}{\left|A^{\prime} B\right|}|A B|^{2}
\end{aligned}
$$

O lo que es lo mismo:

$$
\left|A^{\prime} H^{\prime}\right|=\frac{S\left|A^{\prime} B\right|^{2}}{2\left|B H^{\prime}\right||A B|^{2}}
$$

Dado que el angulo $\angle A H B$ es recto tenemos:

$$
\left|A^{\prime} B\right|^{2}=\left|A^{\prime} H^{\prime}\right|^{2}+\left|B H^{\prime}\right|^{2}=\left|B H^{\prime}\right|^{2}+\frac{S^{2}\left|A^{\prime} B\right|^{4}}{4\left|B H^{\prime}\right|^{2}|A B|^{4}}
$$

O de otra forma, teniendo en cuenta que el vector $\overrightarrow{A B}$ es unitario por construcción y por tanto $|A B|=1$ :

$$
4\left|B H^{\prime}\right|^{4}-4\left|A^{\prime} B\right|^{2}\left|B H^{\prime}\right|^{2}+S^{2}\left|A^{\prime} B\right|^{4}=0
$$

Se trata de una ecuación de segundo grado en $\left|B H^{\prime}\right|^{2}$ cuyas soluciones son:

$$
\left|B H^{\prime}\right|=\left|A^{\prime} B\right| \sqrt{\frac{1 \pm \sqrt{1-S^{2}}}{2}}
$$


Dichas soluciones corresponden al valor de las dos diagonales del rombo $A B C D$ de la figura B.1. Es fácil de ver que la diagonal más pequeña (que es la que se utiliza para SALI) corresponde al valor negativo del radicando.

Por otra parte, de nuevo de la figura B.1 se puede inferir a partir de la semejanza de los triángulos $A H B$ y $A^{\prime} H^{\prime} B$ que:

$$
S A L I=2|B H|=\frac{2\left|B H^{\prime}\right|}{\left|A^{\prime} B\right|}
$$

Todas estas manipulaciones nos permiten expresar el valor del indicador en términos geométricos como:

$$
S A L I=\sqrt{2\left(1-\sqrt{1-S^{2}}\right.}
$$

Dado que el resultado debe ser un número real sabemos que $S^{2} \leq 1$. De hecho $S$ tiene que ser bastante pequeño puesto que consideramos solo un entorno reducido de $z_{0}$, por tanto podemos utilizar la aproximación:

$$
\sqrt{1-S^{2}} \sim 1-\frac{S^{2}}{2}
$$

Obteniendo finalmente:

$$
S A L I \sim S
$$

Podemos observar que el valor de SALI que hemos obtenido coincide aproximadamente con el área del rombo normalizado $A B C D$ de la figuras B.1. Esta idea ha sido utilizada por Ch. Skokos [22], para generalizar el indicador SALI usando más vectores de desviación. En este sentido ha definido su nuevo indicador $G A L I_{n}$ como:

$$
G A L I_{n} \sim \frac{V}{|A B|^{n}}
$$

Donde $V$ es el volumen del simplex formado por los $n$ vectores de desviaci'ón $\vec{V}_{i} . S A L I$ sería pues equivalenta a $G A L I_{2}$.

Calcularemos ahora a partir de datos de la órbita el área del rombo $A B C D$ de la figura B.1.

Consideremos la ecuación de variación 2.84 que obtuvimos en la sección 2.4 .

$$
\frac{d \vec{v}_{1}(t)}{d t}=J \nabla^{2} H(\vec{z}(t)) \vec{v}_{1}(t)
$$


En un entorno reducido del punto $z_{0}$ la correspondiente matriz de monodromía será la matriz constante:

$$
M=\left.J \nabla^{2} H(\vec{z}(t))\right|_{z=z_{0}}
$$

Esto permite calcular sus valores propios $\lambda_{i}$ que serán en general números complejos.

Ordenemos los valores propios $\lambda_{i}$ de forma que se cumpla:

$$
i \leq j \equiv \operatorname{Re}\left(\lambda_{i}\right) \leq \operatorname{Re}\left(\lambda_{j}\right)
$$

Se puede demostrar [22] que estos valores propios son opuestos dos a dos es decir $\lambda_{i}=-\lambda_{(i+N)}$.

En un entorno reducido del punto $z_{0}$ la ecuación 2.84 se comporta pues aproximadamente como un sistema de ecuaciones diferenciales lineales ordinarias con coeficientes constantes. Este tipo de sistemas tiene como solución:

$$
\vec{v}(t)=\sum_{i=1}^{2 N} v_{i} e^{\lambda_{i} t} \vec{u}_{i}
$$

Donde los vectores $\vec{u}_{i}$ forman una base ortonormal de dimensión $2 N$ convenientemente elegida.

De acuerdo con la definición del producto vectorial y la figura B.1, tenemos:

$$
S=\left|\hat{v}_{1} \times \hat{v}_{2}\right|
$$

Donde hemos hecho $\hat{v}_{i}=\frac{\vec{v}_{i}}{\left|\vec{v}_{i}\right|} \quad i=1,2$. Lo que nos permite reescribir B.13 como:

$$
S=\left|\frac{\vec{v}_{1} \times \vec{v}_{2}}{\left|\vec{v}_{1}\right|\left|\vec{v}_{2}\right|}\right|
$$


Efectuando el producto vectorial tenemos:

$$
\vec{v}_{1} \times \vec{v}_{2}=\sum_{i=1}^{2 N} v_{1 i} e^{\lambda_{i} t} \vec{u}_{i} \times \sum_{i=1}^{2 N} v_{2 i} e^{\lambda_{i} t} \vec{u}_{i}
$$

De forma equivalente:

$$
\vec{v}_{1} \times \vec{v}_{2}=\left(\sum_{k=1}^{2 N}\left(C_{k} e^{\left(\lambda_{i}+\lambda_{j}\right) t} \vec{u}_{k}\right)\right.
$$

Donde hemos supuesto que $\vec{u}_{i} \times \vec{u}_{j}=\vec{u}_{k}$ y que $C_{k}=v_{1 i} v_{2 j}-v_{2 i} v_{1 j}$ Por tanto:

$$
\left|\vec{v}_{1}(t) \times \vec{v}_{2}(t)\right|=\sqrt{\sum_{k=1}^{2 N} C_{k}^{2} e^{2\left(\lambda_{i}+\lambda_{j}\right) t}} i \neq j
$$

Consideremos dos casos:

- $\operatorname{Re}\left(\lambda_{1}\right) \neq 0$.

En este caso podemos razonar de la siguiente forma:

$$
\begin{aligned}
& \left|\vec{v}_{1}(t) \times \vec{v}_{2}(t)\right|=\sqrt{\left.C_{1}^{2} e^{2\left(\lambda_{1}+\lambda_{2}\right) t}\right)+\sum_{2}^{2 N}\left(C_{i}^{2} e^{2\left(\lambda_{i}+\lambda_{j}\right) t}\right)} \\
& \left|\vec{v}_{1}(t) \times \vec{v}_{2}(t)\right|=C_{1} e^{\left(\lambda_{1}+\lambda_{2}\right) t} \sqrt{1+\sum_{2}^{2 N}\left(\frac{C_{i}^{2}}{C_{1}^{2}} e^{2\left(\lambda_{i}+\lambda_{j}-\lambda_{1}-\lambda_{2}\right) t}\right)} \\
& \left|\vec{v}_{1}(t) \times \vec{v}_{2}(t)\right| \sim C_{1} e^{\left(\lambda_{1}+\lambda_{2}\right) t}
\end{aligned}
$$

Dado que $e^{\left.2\left(\lambda_{i}+\lambda_{j}-\lambda_{1}-\lambda_{2}\right) t\right)} \sim 0$ por ser $\operatorname{Re}\left(\lambda_{1}\right)>\operatorname{Re}\left(\lambda_{i}\right) i>2$ y $t$ muy grande. 
Por otra parte sabemos que:

$$
\begin{aligned}
& \left|A^{\prime} B\right|=\left|\vec{v}_{1}(t)\right|=\left|\sum_{1}^{2 N} v_{1 i} e^{\lambda_{i} t} \vec{u}_{i}\right| \\
& \left|A^{\prime} B\right|=\sqrt{v_{11}^{2} e^{2 \lambda_{1} t}+\sum_{2}^{2 N} v_{1 i}^{2} e^{2 \lambda_{i} t}} \\
& \left|A^{\prime} B\right|=v_{1 i} e^{\lambda_{1} t} \sqrt{1+\sum_{2}^{2 N} \frac{v_{1 i}^{2}}{v_{11}^{2}} e^{2\left(\lambda_{i}-\lambda_{1}\right) t}} \\
& \left|A^{\prime} B\right| \sim v_{11} e^{\lambda_{1} t}
\end{aligned}
$$

Analogamente

$$
\begin{aligned}
\left|B C^{\prime}\right| & =\left|\vec{v}_{2}(t)\right|=\left|\sum_{1}^{2 N} v_{2 i} e^{\lambda_{i} t} \vec{u}_{i}\right| \\
\left|B C^{\prime}\right| & =\sqrt{v_{21}^{2} e^{2 \lambda_{1} t}+\sum_{2}^{2 N} v_{2 i}^{2} e^{2 \lambda_{i} t}} \\
\left|B C^{\prime}\right| & =v_{2 i} e^{\lambda_{1} t} \sqrt{1+\sum_{2}^{2 N} \frac{v_{2 i}^{2}}{v_{21}^{2}} e^{2\left(\lambda_{i}-\lambda_{1}\right) t}} \\
\left|B C^{\prime}\right| & \sim v_{21} e^{\lambda_{1} t}
\end{aligned}
$$

Dado que $e^{2\left(\lambda_{i}-\lambda_{1}\right) t} \sim 0$ cuando t es lo suficientemente grande.

Sustituyendo las ecuaciones B.18 ,B.19 y B.20 en la ecuación B.14 tenemos:

$$
\begin{aligned}
& S A L I=S=\left|\frac{\vec{v}_{1} \times \vec{v}_{2}}{\left|\vec{v}_{1}\right|\left|\vec{v}_{2}\right|}\right| \\
& S A L I=\frac{C_{1} e^{\left(\lambda_{1}+\lambda_{2}\right) t}}{v_{11} e^{\lambda_{1} t} v_{21} e^{\lambda_{1} t}} \\
& S A L I=\eta e^{-\left(\lambda_{1}-\lambda_{2}\right) t}
\end{aligned}
$$

Donde hemos agrupado en $\eta$ todas las constantes pertinentes.

Por tanto se puede afirmar que:

$$
S A L I=O\left(e^{-\lambda t}\right) \quad \lambda>0
$$

- $\operatorname{Re}\left(\lambda_{i}\right)=0$. 
Es decir todos los valores propios de la matriz de monodromía son de la forma $\lambda_{i}=i \omega_{i}$. Por otra parte, como ya hemos indicado, los valores propios $\lambda_{i}$ son opuestos dos a dos $\left(\lambda_{i}=-\lambda_{i+N}\right)$. Esto implica que al ser imaginarios podemos expresar el producto vectorial $\vec{v}_{1} \times \vec{v}_{2}$ de la forma:

$$
\vec{v}_{1} \times \vec{v}_{2}=\sum_{1}^{N} \alpha_{k} \cos \left(\left(\omega_{i}+\omega_{j}\right) t+\psi_{k}\right) \vec{u}_{k} \quad i \neq j
$$

Donde hemos supuesto que $\vec{u}_{i} \times \vec{u}_{j}=\vec{u}_{k}$.

De forma análoga tenemos:

$$
\begin{aligned}
\left|A^{\prime} B\right| & =\sqrt{\sum_{k=1}^{N} \beta_{k}^{2} \cos ^{2}\left(\omega_{k} t+\psi_{k}\right)} \\
\left|B C^{\prime}\right| & =\sqrt{\sum_{k=1}^{N} \gamma_{k}^{2} \cos ^{2}\left(\omega_{k} t+\psi_{k}\right)}
\end{aligned}
$$

Es evidente que $|A B|,|B C| \neq 0$, dado que los puntos $A, B$ y $C$ pertenecen a órbitas distintas. Por tanto B.7 siempre tiene sentido. Por otra parte $\cos ^{2}(\omega t) \leq 1$ y por tanto:

$$
\begin{aligned}
& S^{2}=\sum_{1}^{N} \alpha_{k}^{2} \cos ^{2}\left(\left(\omega_{i}+\omega_{j}\right) t+\psi_{k}\right) \leq \sum_{1}^{N} \alpha_{k}^{2} \leq V \\
& \left|A^{\prime} B\right|^{2}=\sum_{k=1}^{N} \beta_{k}^{2} \cos ^{2}\left(\omega_{k} t+\psi_{k}\right) \leq \sum_{1}^{N} \beta_{k}^{2} \leq W \\
& \left|B C^{\prime}\right|^{2}=\sum_{k=1}^{N} \gamma_{k}^{2} \cos ^{2}\left(\omega_{k} t+\psi_{k}\right) \leq \sum_{1}^{N} \gamma_{k}^{2} \leq W^{\prime}
\end{aligned}
$$

Donde $V, W$ y $W^{\prime}$ son números finitos no nulos.

Por tanto tenemos:

$$
\begin{aligned}
& S A L I=\sqrt{2\left(1-\sqrt{\left.1-S^{2}\right)}\right.} \sim S \\
& S A L I \leq \frac{\sqrt{V}}{W W^{\prime}}<\infty
\end{aligned}
$$


Por tanto se puede afirmar que:

$$
S A L I=O(1)
$$

Mostremos que este caso corresponde a una órbita regular.

Para un tiempo $T$ arbitrariamente grande tenemos:

$$
\begin{aligned}
& S A L I=O(1) \equiv|A B| \leq W \\
& \log \left(\left|A^{\prime} B\right|\right) \leq \log (W) \\
& \frac{1}{T} \log \left(\left|A^{\prime} B\right|\right) \leq \frac{1}{T} \log (W) \\
& \lim _{T \rightarrow \infty} \frac{1}{T} \log \left(\left|A^{\prime} B\right|\right) \leq \lim _{T \rightarrow \infty} \frac{1}{T} \log (W)=0
\end{aligned}
$$

Recordando la definición del coeficiente de Lyapunov, tenemos:

$$
L C E=\lim _{T \rightarrow \infty} \frac{1}{T} \log \left(\left|A^{\prime} B\right|\right) \leq 0
$$

Que corresponde a una órbita regular de acuerdo con el criterio de Lyapunov.

Dado que los dos casos que hemos analizado son exhaustivos y se excluyen entre si, se puede deducir que el primer caso corresponde a una órbita caótica. 


\section{Apéndice $\mathrm{C}$}

\section{Detalle de implementación}

El sistema esta implementado en fortran 90 sobre la base de los siguientes grandes bloques.

\section{C.1. Descripción del Sistema}

Se trata de la única parte del software que es especifica del sistema que se ha estudiado (en este trabajo básicamente LiCN y Henon-Heiles)

Se compone de los siguientes módulos.

- Posición Implementa las variables necesarias para describir un estado instantáneo del sistema.

- Potencial Describe la energía potencial a la que esta sometido el sistema.

- Ecuaciones del movimiento. Implementa las ecuaciones de Hamilton

\section{C.2. Integrador}

Las ecuaciones Eq. (4.10) y Eq. (4.11) solo pueden ser integradas numéricamente, al ser un conjunto de ecuaciones diferenciales no lineales altamente acopladas.

Se trata de la parte del software que se utiliza para integrar las ecuaciones del movimiento.

En el proceso de este desarrollo se han probado las siguientes alternativas.

- Runge-Kutta-Merston de paso variable

- Metodo simplectico particionado 
Tabla C.1: Comparativa de Métodos de Integración

\begin{tabular}{llr} 
Cod. & Método & Variación de la Energia \\
\hline a & Gear & $10^{-13}$ \\
b & Runge Kutta 4 & $10^{-12}$ \\
c & Runge Kutta Felhberg-78 & $10^{-12}$ \\
d & Runge Kutta Merston & $10^{-12}$ \\
\hline
\end{tabular}

- Gear

cuyos resultados se resumen en la tabla C.1

La descripción de dichos metodos se ha tomado de [42].

Aunque los módulos de este bloque utilizan la descripción del sistema, se han escrito de forma que sean totalmente independientes de los mismos. Esto nos facilita (en la medida de lo posible el que se pueda cambiar el objeto de estudio simplemente cambiando la descripción del sistema.

El resultado de todas estas pruebas es que el cálculo de este indicador no resulta muy afectado por el tipo de integrador que se utilice.

\section{C.3. Indicadores}

En este bloque implementamos diversos indicadores:

- Superficie de Sección de Poincare

- SALI

- Analisis de Frecuencia

De nuevo es importante destacar que aunque se utiliza el resto de los módulos este desarrollo es independiente de ellos.

\section{C.4. Aplicaciones}

Se trata de pequeños programas que se limitan a llamar a los modulos anteriores. Son responsables en último término de los resultados expuestos en este trabajo. Dichas aplicaciones básicamente se dedican a generar condiciones iniciales seleccionadas, calcular a partir de ellas orbitas y a partir de ellas calcular los distintos indicadores.

- TRAYECTORIAS A partir de condiciones iniciales escogidas se generar trayectorias individuales de las que obtiene el comportamiento dinámico de SALI 
- FRECUENCIA Realiza un mapa de frecuencia de una muestra de trayectorias seleccionadas y al mismo tiempo calcula sus SALI correspondientes.

- SECCIONES Realiza las superficies de sección de Skokos-

El resultado de estos aplicaciones se almacena en ficheros secuénciales para explotarlos posteriormente con mediante el uso de herramientas graficas y estadisticas.

\section{C.5. Graficos}

Se ha utilizado scripts en $\mathrm{R}$ para la confección de la totalidad de los gráficos utilizados en este trabajo 